Linköping Studies in Science and Technology

Dissertation No. 2012

\title{
Learning Human Gait
}

\section{Parinaz Kasebzadeh}

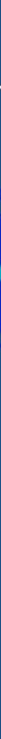


Linköping studies in science and technology. Dissertations.

No. 2012

\section{Learning Human Gait}

\section{Parinaz Kasebzadeh}


Cover illustration: Human gait signature for running (front) and walking (back) motion modes extracted from experimental data as described in Chapter 6. The footprints are only for illusterative purposes and do not represent the exact gait cycles.

Linköping studies in science and technology. Dissertations.

No. 2012

\section{Learning Human Gait}

Parinaz Kasebzadeh

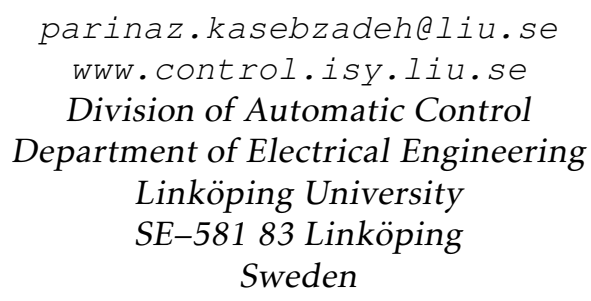

ISBN 978-91-7519-014-3

ISSN 0345-7524

Copyright @ 2019 Parinaz Kasebzadeh

Printed by LiU-Tryck, Linköping, Sweden 2019 
To Kamiar! 



\section{Abstract}

Pedestrian navigation in body-worn devices is usually based on global navigation satellite systems (GNSS), which is a sufficient solution in most outdoor applications. Pedestrian navigation indoors is much more challenging. Further, GNSS does not provide any specific information about the gait style or how the device is carried. This thesis presents three contributions for how to learn human gait parameters for improved dead-reckoning indoors, and to classify the gait style and how the device is carried, all supported with extensive test data.

The first contribution of this thesis is a novel approach to support pedestrian navigation in situations when GNSS is not available. A novel filtering approach, based on a multi-rate Kalman filter bank, is employed to learn the human gait parameters when GNSS is available using data from an inertial measurement unit (IMU). In a typical indoor-outdoor navigation application, the gait parameters are learned outdoors and then used to improve the pedestrian navigation indoors using dead-reckoning methods. The performance of the proposed method is evaluated with both simulated and experimental data.

Secondly, an approach for estimating a unique gait signature from the inertial measurements provided by IMU-equipped handheld devices is proposed. The gait signatures, defined as one full cycle of the human gait, are obtained for multiple human motion modes and device carrying poses. Then, a parametric model of each signature, using Fourier series expansion, is computed. This provides a low-dimensional feature vector that can be used in medical diagnosis of certain physical or neurological diseases, or for a generic classification service outlined below.

The third contribution concerns joint motion mode and device pose classification using the set of features described above. The features are extracted from the received IMU gait measurement and the computed gait signature. A classification framework is presented which includes standard classifiers, e.g. Gaussian process and neural network, with an additional smoothing stage based on hidden Markov model.

There seems to be a lack of publicly available data sets in these kind of applications. The extensive datasets developed in this work, primarily for performance evaluation, have been documented and published separately. In the largest dataset, several users with four body-worn devices and 17 body-mounted IMUs performed a large number of repetitive experiments, with special attention to get well annotated data with ground truth position, motion mode and device pose. 



\section{Populärvetenskaplig sammanfattning}

Våra smarta mobiler och klockor har idag sensorer som håller koll på var vi är och hur vi rör oss. Satellit-baserade positioneringssystem ger positionen där vi är, och förflyttningshastighet ger tillsammans med mätningar från accelerometrar information om hur vi rör oss (går, springer, cyklar, åker motorfordon). Detta finns idag inbyggt i mjukvaran. En svaghet i dagens lösningar är att positioneringen slutar fungera inomhus och överallt dit de svaga satellitsignalerna inte når oss. En möjlighet är att använda mer sofistikerade algoritmer för att analysera vår gångstil för både medicinska tillämpningar och som en service till andra applikationer.

I ett första bidrag presenteras en metod för hur man kan lära sig steglängd och hur tungt man sätter ner fötterna under perioder man går utomhus och har tillgång till satellitnavigering. Dessa parametrar kan sedan användas inomhus för att räkna antal steg med hjälp av accelerometrar. Tillsammans med gyroskop och magnetometrar, som också finns i de flesta smarta telefoner, kan man med s.k. dödräkning bestämma hur man gått inomhus med en betydligt mindre drift över tiden jämfört med konventionell dödräkning där man inte tar hänsyn till individuella gångstils-parametrar.

I ett andra bidrag studeras hur gångstilen kan karaktäriseras på detaljnivå. Detta görs genom att studera steg för steg hur accelerometer-signalen varierar i stegcykeln. Med Fourieranalysteknik kan man beskriva en individuell gångstill med några tiotal parametrar och från dessa dra slutsatser om hur personen rör sig (går, joggar eller springer), var telefonen är placerad (i handen, i fickan, i en väska eller ryggsäck). Det tredje bidraget använder dessa parametrar för att jämföra ett antal moderna maskininlärningsmetoder för att klassificera gångstil och var telefonen är belägen på kroppen. I framtida bidrag kan dessa parametrar korreleras mot olika skador eller neurologiska sjukdomar som ger upphov till hälta eller andra asymmetrier i gångstilen för medicinsk diagnostik. 



\section{Acknowledgments}

The five-year PhD journey, with all its ups and downs is coming to an end. It has been an enjoyable experience with endless learning opportunities yet challenging surprises along the way. Luckily, I haven't been alone during the whole journey and have had the chance to meet many people who helped me along the way. The least I can do is to take this opportunity to thank them for all their supports.

Typically, in dissertation theses, the main supervisors are acknowledged first. I would also like to do that but not to follow the crowd but since I genuinely believe that Fredrik Gustafsson is the one with the highest impact factor in this chapter of my life. It might be expected that a supervisor has inspiring ideas. Of course, that holds for Fredrik too, but what I most appreciate is his endless support and reassuring words during this time particularly when the things weren't going the way they should have gone! Thanks Fredrik!

Through my doctoral studies, I became privileged to have Gustaf Hendeby as my co-supervisor. Besides being a diligent supervisor, able to motivate me to success, he has also been a great friend. Thanks Gustaf for the many hours that you devoted to the questions, concerns, stressful situations and basically everything that I brought to you.

I also would like to thank Martin Enqvist, Svante Gunnarsson and Ninna Stensgård for providing us an extraordinary work place. Automatic Control has an impeccable environment and this would not be possible without great colleagues. Thank you all for making such a friendly and amazing atmosphere at work. I also would like to gratefully acknowledge the European Union FP7 Marie Curie training program on Tracking in Complex Sensor Systems (TRAX) and the Swedish Research Council project Scalable Kalman Filter for the financial support.

Special thanks also go to the proofreading gang, Kamiar Radnosrati, Erik Hedberg, Du Ho, Shervin Parvini Ahmadi, it goes without saying how grateful I am for your comments and time.

A big thanks to my amazing friends, I cannot express how much grateful I am for having you in my life to share both the good moments and bad. Thanks for all of the support you have given me!

Mom, Dad, I always appreciate your endless love and your support throughout my life. Thank you for believing in me and keeping me focused on my goals even when we live miles apart. You know how much I love you! Pedi, you are the best brother ever, thanks for bringing so much fun into our life!

Here I am again, the toughest one for last. Thank you Kamiar, life cannot get any better when you are around. Definitely, I could not have come this far without you and all your support! I am so looking forward to beginning a new chapter of our life together! Love you!

Linköping, August 2019

Parinaz Kasebzadeh 



\section{Contents}

Notation

1 Introduction 1

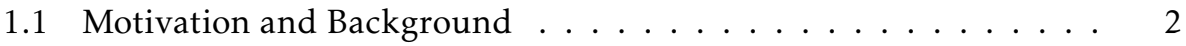

1.2 Pedestrian Dead Reckoning Positioning . . . . . . . . . . . 3

1.3 Motion and Device Mode Classification . . . . . . . . . . . 5

1.3.1 Asynchronous Averaging of Gait Cycles . . . . . . . . . 8

1.3.2 Joint Pedestrian Motion State and Device Pose Classification 9

1.4 Contributions and Publications . . . . . . . . . . . 10

1.5 Thesis Outline . . . . . . . . . . . . . . . . . . . 12

\section{Background}

2 Pedestrian Dead Reckoning Positioning 17

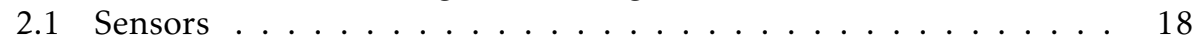

2.1.1 Inertial Measurements . . . . . . . . . . . . . . . 18

2.1.2 Supporting Measurements . . . . . . . . . . . . . 20

2.1 .3 Sensor Fusion . . . . . . . . . . . . . . . . . 21

2.2 Model Framework . . . . . . . . . . . . . . . . . . . . . 22

2.3 State-Space Estimation . . . . . . . . . . . . . . 22

2.3.1 Stochastic State Space Models . . . . . . . . . . . . . 23

2.3.2 State Estimation . . . . . . . . . . . . . . . . . 25

2.3.3 Kalman Filter . . . . . . . . . . . . . . . . . . 27

2.3.4 Extended Kalman Filter _. . . . . . . . . . . . . . . 28

2.3.5 Rauch-Tung-Striebel Smoother . . . . . . . . . . . . 30

2.3.6 Extended Rauch-Tung-Striebel Smoother . . . . . . . . . . . 30

2.4 Hidden Markov Model . . . . . . . . . . . . . . . . . . . 31

2.5 Summary .................... 32

3 Statistical Machine Learning 33

3.1 Basic Concepts . . . . . . . . . . . . . . . . . . . 33

3.2 Classification Methods . . . . . . . . . . . . . . 34 
3.2.1 Weighted $K$-Nearest Neighbor . . . . . . . . . . . . 35

3.2.2 Gaussian Process ...................... 36

3.2.3 Feed Forward Neural Network . . . . . . . . . . . . . 38

3.3 Temporally Correlated Classes . . . . . . . . . . . . . . . . 41

3.4 Summary ......................... 42

\section{Applications}

4 Gait Parameter Estimation $\quad 45$

4.1 Pedestrian Odometry . . . . . . . . . . . . . . . . 46

4.1.1 Extended Pedestrian Odometric Model . . . . . . . . . . . . 46

4.1.2 Horizontal Model . . . . . . . . . . . . . . . . . . 47

4.2 Step Detection . . . . . . . . . . . . . . . . . 51

4.2.1 Step Detection Algorithm . . . . . . . . . . . . 52

4.2 .2 Step Length Estimation . . . . . . . . . . . . . . . . . . . . 54

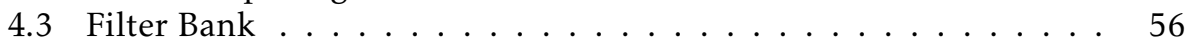

4.3.1 Offline Kalman Filter Bank . . . . . . . . . . . . . . . . 57

4.3.2 Online Kalman Filter Bank . . . . . . . . . . . . . . . . . . 59

4.4 Dead Reckoning in Different Environments . . . . . . . . . . . 59

4.4.1 Simulated Environment . . . . . . . . . . . 62

4.4.2 Real Environment . . . . . . . . . . . . . 63

4.4 .3 Data Collection ................. . . 64

4.4.4 Standing Still Detection . . . . . . . . . . . 72

4.4.5 Motion Mode Classification Using HMM . . . . . . . . . . . 74

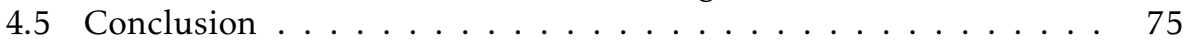

5 IMU Dataset For Motion and Device Mode $\quad 77$

5.1 Experiment Setup . . . . . . . . . . . . . . . . 77

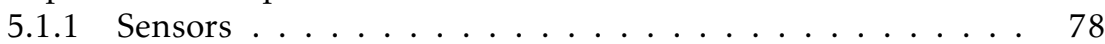

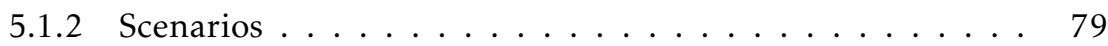

5.1 .3 Participants ...................... 82

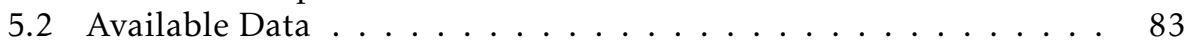

5.2 .1 Collected Data . . . . . . . . . . . . . . . 83

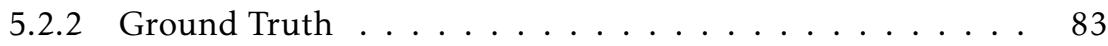

5.2 .3 Acquire Data . . . . . . . . . . . . . . 85

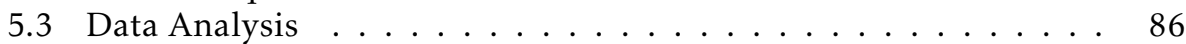

5.3 .1 Feature Extraction . . . . . . . . . . . . . . 86

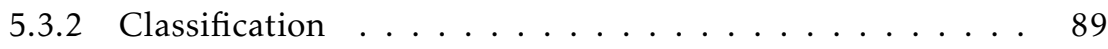

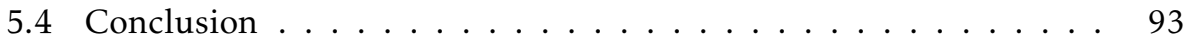

6 Asynchronous Averaging of Gait Cycles 95

6.1 Problem Formulation and Notation . . . . . . . . . . . . . . . 96

6.2 Optimal Segmentation of Gait Cycles . . . . . . . . . . . . . . . . 97

6.2.1 Solution for Optimization Problem . . . . . . . . . . . 98

6.2.2 Classical Gait Segmentation . . . . . . . . . . . . . . . . 99

6.3 Data Reduction with Fourier Series . . . . . . . . . . . . 100 
6.4 Experimental Results ... . . . . . . . . . . . . 103

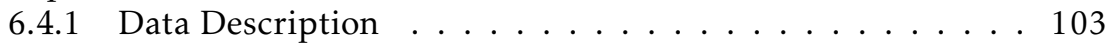

6.4 .2 Performance Evaluation ................ 104

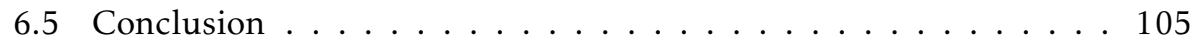

7 Joint Pedestrian Motion State and Device Pose Classification 113

7.1 Problem Formulation . . . . . . . . . . . . . . . . . 113

7.1.1 Gait Segmentation . . . . . . . . . . . . . 114

7.1 .2 Feature Extraction . . . . . . . . . . . . . 116

7.1.3 Generating the Dataset . . . . . . . . . . . . 118

7.2 Experimental Results . . . . . . . . . . . . . . . . 118

7.2.1 Coarse Classification . . . . . . . . . . . . . 119

7.2.2 Fine Classification . . . . . . . . . . . . . . 121

7.3 Conclusions .......................... 125

\section{Conclusion and Future Work}

8 Summary and Future Work 129

8.1 Summary of Contribution . . . . . . . . . . . . . . . 129

8.2 Future Work . . . . . . . . . . . . . . . 130

$\begin{array}{lr}\text { Bibliography } & 133\end{array}$ 



\begin{tabular}{cl} 
AbBREVIATION & \\
\hline Abbreviation & Meaning \\
\hline CT & Coordinated turn \\
CV & Constant velocity \\
EKF & Extended Kalman filter \\
ERTSS & Extended Rauch-Tung-Striebel smoother \\
GNSS & Global Navigation Satellite System \\
GP & Gaussian process \\
HMM & Hidden Markov model \\
IPS & Indoor positioning system \\
IMU & Inertial measurement unit \\
INS & Inertial navigation system \\
IMM & Interacting multiple model \\
KF & Kalman filter \\
KNN & K-nearest neighbor \\
LSE & Least squares estimator \\
LTE & Long-term evolution \\
LOS & Line of sight \\
MLE & Maximum likelihood estimator \\
MEMS & Micro-machined electromechanical systems \\
PDR & Pedestrian dead reckoning \\
PNS & Pedestrian Navigation System \\
RSS & Received signal strength \\
RTSS & Rauch-Tung-Striebel smoother \\
SSM & State-space model \\
\hline
\end{tabular}





\section{Mathematical Style}

\begin{tabular}{cl}
\hline Notation & Meaning \\
\hline$z$ & Scalar parameter or variable \\
$\mathbf{Z}$ & Parameter or variable vector \\
$\mathbf{Z}$ & Parameter or variable matrix \\
\hline
\end{tabular}

\section{SYMBOLS AND OPERATIONS}

\begin{tabular}{|c|c|}
\hline Notation & Meaning \\
\hline $\mathbf{x}_{k}$ & State vector at time $k$ \\
\hline $\mathbf{x}_{1: k}$ & Set of states from time 1 to $k$ \\
\hline $\mathbf{u}_{k}$ & Known input vector at time $k$ \\
\hline $\mathbf{y}_{k}$ & Measurements at time $k$ \\
\hline $\mathbf{y}_{1: k}$ & Set of measurements from time 1 to $k$ \\
\hline $\mathbf{f}_{k}(\cdot)$ & State update equation at time $k$ \\
\hline $\mathbf{h}_{k}(\cdot)$ & Measurement equation at time $k$ \\
\hline$\hat{\mathbf{x}}_{k \mid k}$ & $\begin{array}{l}\text { State estimate at time } k \text { given measurements up to and } \\
\text { including time } k\end{array}$ \\
\hline $\mathbf{P}_{k \mid k}$ & $\begin{array}{l}\text { State covariance at time } k \text { given measurements up to } \\
\text { and including time } k\end{array}$ \\
\hline$\hat{\theta}$ & Parameter estimate \\
\hline $\mathcal{N}(\mu, \Sigma)$ & Gaussian distribution with mean $\mu$ and covariance $\Sigma$ \\
\hline $\arg \max$ & Maximizing argument \\
\hline $\arg \min$ & Minimizing argument \\
\hline$|\cdot|$ & Euclidean norm of a vector \\
\hline$\|\cdot\|$ & $L_{2}$ norm \\
\hline$p(a \mid b)$ & Conditional PDF of stochastic variable \\
\hline $\operatorname{Cov}(\cdot)$ & Covariance \\
\hline $\mathbf{E}(\cdot)$ & Expected value \\
\hline $\mathbb{R}$ & Set of real numbers \\
\hline$s_{n}$ & Sample times of IMU \\
\hline$M$ & Number of gait cycles \\
\hline$t_{m}$ & Step time for $m$ :th gait cycle \\
\hline$\tau$ & Normalized time $\tau \in[0,1)$ \\
\hline$\hat{\boldsymbol{g}}_{m}(\tau)$ & $m:$ th gait cycle \\
\hline$\epsilon_{\mathrm{lo}}$ & Minimum gait cycle time \\
\hline$\epsilon_{\text {up }}$ & Maximum gait cycle time \\
\hline$\epsilon_{p}$ & Peak threshold \\
\hline$\epsilon_{v}$ & Valley threshold \\
\hline$\overline{\boldsymbol{g}}(\tau)$ & Gait signature \\
\hline$\hat{G}[l]$ & Fourier series expansion of $\overline{\boldsymbol{g}}(\tau)$ \\
\hline$n_{c}$ & Number of classes \\
\hline$n_{r}$ & Size of training set \\
\hline$n_{d}$ & Size of development set \\
\hline
\end{tabular}




\section{1}

\section{Introduction}

Locating a mobile user with a pedestrian navigation system (PNS), specifically when direct measurement of the position is not always available, relies on inferring orientation and position from other sources of information. For example, short range radio network positioning systems which typically rely on the ranging measurements and signal strength have been utilized in many studies. These systems are infrastructure-based, require an accurate radio map of the environment and rely on a large number of beacon nodes.

Emerging cheap and light-weight sensors have increased the importance of applications enabled by PNS. Consequently, alternative solutions that could address the existing problems are proposed in the literature. Such applications include, but are not limited to, pure navigation and guidance tools, healthcare assistance systems to infotainment applications, and more generally in location based services.

The pedestrian dead reckoning (PDR) principle is an alternative PNS solution, which relies on a small number of sensors being carried by the mobile user. PDR attains position and orientation information from sensory data by detecting when the human makes steps and how the direction changes between footsteps. The performance of PDR algorithms is highly dependent on the accuracy of such human gait information.

Various systems and algorithms for PNSs have been introduced in the literature. Comparing them shows that PDR using inertial measurement units (IMUs) has attracted the most interest as it imposes no extra cost and does not rely on additional infrastructure. Besides the large class of IMU-based systems, there are also other approaches that use other sensors such as e.g. electronic pedometers.

PNSs can be generally classified based on the location of the installed sensors. Since body-fixed systems require extra devices to be produced and mounted, hand-held devices gain more interest thanks to the rapid development of smart- 
phones.

In this thesis, we investigate the modeling and estimation problem for pedestrian positioning applications using smartphones. The information obtained from the mobile user can be interpreted as inputs to a gait model which is a function of the user's motion pattern as well as the physical attributes. The gait model parameter estimation problem is investigated in this thesis. Given the gait parameters, a filtering solution is employed to process the gait model and provide an estimate of the user's location.

Improvements in the accuracy of gait modeling can be obtained by incorporating the user's behavior into the model. Considering the user's motion behavior together with the device mode enables us to find a unique gait signature, which represents one full cycle of the human gait. The gait signature, on one hand, allows for adopting the gait model parameters properly. On the other hand, they are also suitable for classification of the gait modes that is an interesting application in itself. In this thesis, the joint classification problem based on inertial sensor measurements using multiple machine learning algorithms and a feedforward neural network is studied.

Part I of this thesis provides the basis for the work in Part II, in which four applications are presented and further studied. The motivation and background of this thesis is introduced in Section 1.1. The considered applications are briefly introduced in Section 1.2 and 1.3. Section 1.4 presents the author's contributions followed by the outline of the thesis given in Section 1.5.

\subsection{Motivation and Background}

Micro-electromechanical system (MEMS) technology is typically used in IMU sensors such as accelerometers and gyroscopes. MEMS technology allows for very light, cheap, and small components with low power consumption making it possible to integrate IMUs in many portable devices trivially. Hence, IMUs are embedded in most smart devices, such as smartphones, smartwatches, and virtual reality headsets.

Pedestrian navigation, motion capture, and bio-mechanical analysis are examples of application areas in which IMUs are used to estimate the movement or track the human motions by using the orientation and position information [20, $24,80,92]$. Pedestrians motion prediction is also crucial in autonomous vehicles to make them aware of other agents' intentions. The author in [15], uses inertial measurements together with other sources of information for modeling and predicting pedestrians behavior in real-world scenarios.

In navigation applications, global positioning system (GPS) has been the conventional solution. However, the reliability of GPS is limited in multiple conditions such as indoors, outdoors with bad weather conditions or urban areas in which the GPS signal is not accessible or has very poor quality. Consequently, pedestrian navigation using IMUs in PNS has become a popular and reliable solution in which no extra infrastructure is required.

In general, PNSs using IMUs are categorized based on the sensor placement. 


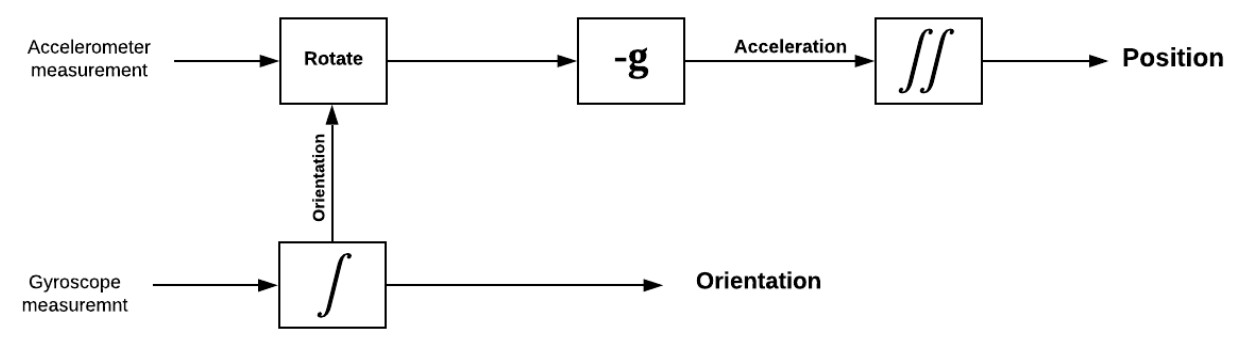

Figure 1.1: Dead reckoning illustration. Attain position and orientation information by integrating the accelerometer and gyroscope measurements.

Hand-held [61, 92, 94, 102], foot-mounted [13, 63], and waist-mounted [5, 58] are the most popular categories that have been considered in the literature. While assuming sensors rigidly attached to different parts of the body relaxes some existing challenges by making the signal pattern more predictable, real-world applications of such systems are extremely limited. Hence, IMUs embedded in smartphones have gained the most interests in pedestrian positioning applications $[24,52,91]$.

Improving the accuracy of parameter estimation requires careful modeling. Moreover, the orientation and position of the smartphone, the so called device mode, should be considered. Device and gait mode recognition using IMUs signal would provide very useful device mode information for estimating gait parameters accurately.

In this thesis, we use measurements collected by sensors and receivers available in most recent smartphones. These measurements are used in different applications. The motivation and background theory for the first and second application are previously published by the author in [48] and in [49]. The third and fourth application are submitted to [50] and [51].

\subsection{Pedestrian Dead Reckoning Positioning}

IMUs consist of several sensors of which a three-axis accelerometer and a threeaxis gyroscope are typically used in pedestrian positioning algorithms. The gyroscope measures angular velocity, and the integration of angular velocity provides orientation information. The earth's gravity and acceleration of the sensor are the quantities measured by accelerometers. Twice integrating acceleration gives the sensor position if the earth's gravity is first properly removed. For removing the earth's gravity, the orientation of the device needs to be known a priori. Hence, the first step in position estimation via inertial system is orientation estimation. Figure 1.1 provides a schematic illustration of the inertial navigation process to attain orientation and position information from inertial sensors, called dead reckoning (DR). 


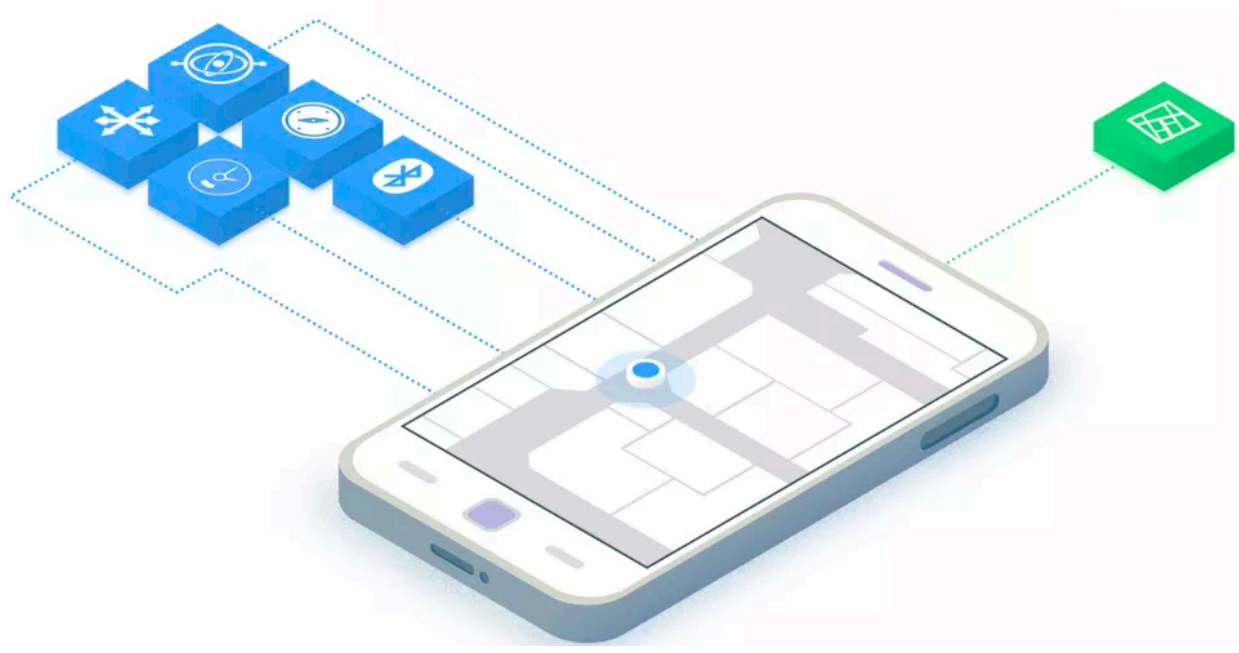

Figure 1.2: Indoor positioning using smartphone. By courtesy of Senion [2].

PDR uses the DR principle in pedestrian navigation system to locate the mobile user in outdoor and/or indoor environments. The PDR algorithms are used to identify when the user takes steps and how the direction changes among the taken steps. Considerable research is conducted on the subject of using IMU signals for PDR $[11,25,41,43,105]$. The step detection problem is typically solved by thresholding techniques, where e.g. the norm of the accelerometer is filtered first and then thresholded [18, 48,67]. These algorithms use accelerometers to estimate gait parameters such as the step length and the number of steps, and the gyroscopes to define the heading. The authors in [104] investigate the PDR using pocket-worn smart phones. Figure 1.2 is an illustration of a smartphone using IMUs, and more sensors, for positioning purposes.

Although IMUs provide accurate motion estimates for short time duration since they have very high sampling rate, they are not self-contained navigation systems and the positioning error drifts over time. Therefore, an IMU algorithm requires supporting measurement to provide accurate estimate of position. In this thesis, whenever the GPS signal is available, the additional information is fused with IMU measurements to improve the estimated pose. More details are presented in Chapter 4.

In order to estimate the gait parameters, it is essential to detect step occurrences and the length of each step. These gait characteristics depend on the walking behavior and the individual's physical attributes. Moreover, even the same person does not have a unique gait under all conditions. Hence, step length is a time-varying property which depends on the speed and frequency of steps. Step 
detection in PDR typically relies on zero velocity update (ZUPT) for the lowerbody mounted IMUs, e.g. foot-mounted applications [67, 86, 101]. ZUPT assumes that when the foot is at rest, at least for a short while in each stance, the bias in the gyroscope and the accelerometer can be directly observed, hence compensated for. The elimination of the bias allows the use of dead-reckoning principles to integrate acceleration and angular rate into a precise trajectory.

However, extra care must be taken when dealing with upper-body sensors. The upper-body mounted or hand-held IMUs might report continuous or unexpected motion while the sensors in the lower body capture the foot at rest behavior. Hence, instead of finding zero velocity periods as with ZUPT, step detection in PDR normally relies on thresholding based peak detection $[46,78]$. Using a fixed threshold typically leads to systematic errors for people heavier or lighter than the test subjects the threshold was designed for. In addition to this systematic error, there are also false positive and negative step detections on the test subjects themselves. To take this relation into consideration, a few parameters such as height and weight should be calibrated before beginning measurement and performing gait parameter estimation. Previous studies on this problem, use constant pre-learned parameters in their models. For instance, in [85], the step frequency and variation of the acceleration is considered with a pre-learned constant parameter. For online step length estimation, a linear relation between the measured frequency of steps and a pre-learned constant parameter is introduced in [57]. In a recent study, the authors in [30] use machine learning approaches such as Gaussian process and neural networks to estimate the user's velocity from IMU measurements collected from devices placed in the pocket. More details about these systems will be presented in Section 4.2.

In Chapter 4, a filtering approach is proposed that can learn gait parameters of the PDR algorithm including the step detection threshold and step length. The proposed approach utilizes a multi-rate Kalman filter bank that estimates the gait parameters when position measurements from GPS are available. This method improves the PDR in time intervals when GPS position estimates are unavailable. An example of this use case is moving from outdoors, with good GPS coverage, to indoors where GPS is unavailable.

Step length is one important gait parameter, which requires occasional absolute position measurements such as GPS to be accurately estimated. In the next subsection, it is discussed how some other gait parameters can be extracted, even completely without any position information.

\subsection{Motion and Device Mode Classification}

The main purpose of a PNS is to obtain a reliable and accurate position estimate, however there are certain metadata which can provide extra information in itself. In this application, the problem of classifying the gait mode (walking, standing still, running) and the device mode (hand-held in view, hand-held in swinging hand, in front/back pocket, and in a backpack) is studied.

Gait analysis and activity monitoring are among the most informative fea- 
tures to be considered in location analysis such as collapse detection, monitoring athletes' activity, balance control evaluation, animal activity tracking, pedestrian navigation, etc. Typically, all these applications rely on IMU sensors to log the activity information $[3,21,22,100]$. Lack of accuracy in gait detection will lead to inaccurate step count and inaccurate estimated step length which in turn will provide a pedestrian position estimate with large bias. One source of information that is beneficial for accurate position estimation or activity analysis is the knowledge of gait mode and device mode that is typically acquired from accelerometer and gyroscope sensor reading $[11,43,58,65]$.

The gait mode is an essential feature in healthcare and sport applications. In addition, the gait mode can choose a set of appropriate internal parameters in the PNS, such as step detection thresholds and step length [48]. In outdoor applications, for example an energy demanding GPS fix can be attained. Here, the objective is to know whether the user is walking, standing still or running. The same compromises about using extra information sources from infrastructure can be applied to indoor PNS. For certain personnel such as rangers and guards, running might show danger and a sudden and unexpected stand still can indicate an accident. In both cases, officers can be automatically alerted.

Gait mode classification using body mounted or wearable IMUs is an attractive area for many researchers [23, 77, 80]. A hidden Markov model (HMM) based method using chest-mounted IMUs for gait analysis and activity classification is proposed in [68]. Portable devices already equipped with IMUs such as smartphones, tablets, or smartwatch, however, attract more interests for activity recognition $[21,26,27,29]$. For a thorough survey of the existing results on recognition of various gait modes see [27].

The device mode is important for the performance gait parameters estimation and design of a PNS $[55,96]$. For instance, one assumption is that the device is rigidity attached to a foot $[13,31,63]$. Other assumptions are that the IMU is fixed on the waist instead of the foot $[5,58]$, located in the front pocket [89], carried horizontally in hand [35], or carried in hand not necessarily horizontally $[61,92,94,102]$. The measured accelerometer and gyroscope magnitudes are considered for classifying four different smartphone modes in [55] and the $86 \%$ classification accuracy is reported. Machine learning approaches have been considered to identify the activity mode and device placement simultaneously in [102] in which various frequency and time domain features are extracted to create the model that fits the data best. The classification rate is evaluated based on the device pose irrespective of the motion activities.

Classification of multiple motion modes can be one step forward into more realistic situations in which the smartphone is permitted to switch arbitrarily between different device modes, as normal users operate their smartphones. Few studies in the literature are performed in relation to the classification of both motion and device modes. The classifier presented in [70] is based on magnetic field and acceleration data which is recorded with a hand-held device. The authors in [90] investigate walking pattern and standing still motion modes. The study is extended by including the running mode in [102]. The motion mode classification is further investigated in $[26,27,76,91]$. 


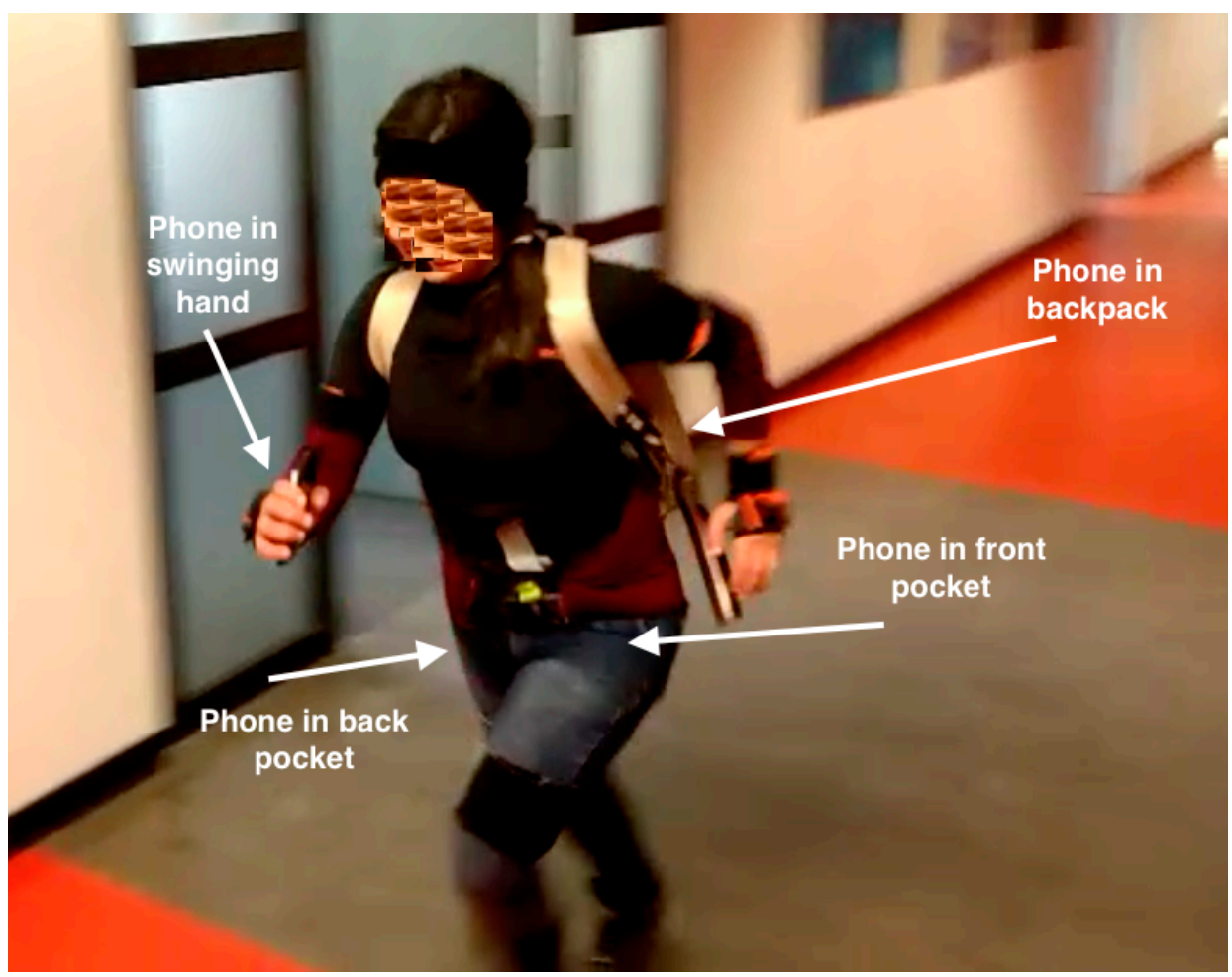

Figure 1.3: Photo from measurement campaign. Activity recognition, the subject is running while carrying four smartphones in swinging hand, in her front and back pocket, and also in her backpack.

The benefits of the mode classification for PNS can be explained as follows. The key design parameters contain the step length and the step detection threshold estimation when the magnitude of the measured acceleration is considered to be caused by a step. Both of these depend on the motion mode. Typically, the smaller the step length the smaller the threshold needs to be. In fact, the device mode can simplify the model further. For example, if the device is hand held flat, the heading relating to the projection (rotation) to the horizontal plane (heading) can be computed by just integrating the angular rate around the gravity vector. In addition, accurate step detection, requires joint gait mode and device mode classification in order to get a proper threshold for the peak detection $[41,69,102,103]$. An adaptive gait detection and step length estimation, based on walking speed classification, is proposed in [41]. A weighted context-based step length estimation algorithm using waist-mounted IMUs embedded in smartphone is proposed in [65] which strives to classify six different pedestrian activities.

Chapter 5 presents an extensive data set in which several users contributed. Different smartphones were carried in several ways, as well as IMUs mounted 
in a body suit. Figure 1.3 shows a subject while collecting data wearing a suit, provided by Xsens, containing the IMU sensors and carrying several smartphones. More details about the extracted features and preliminary classification result are presented in this chapter.

Learning these mode parameters is a completely separate problem from the step length estimation problem in Section 1.2, still using the same IMU data (but no GPS).

\subsubsection{Asynchronous Averaging of Gait Cycles}

Existing approaches for step detection and gait cycle segmentation, typically, rely on measurements collected from hand-held devices such as smartphones that are already equipped with IMUs $[46,59]$. However, due to the large number of affecting factors on the sensor readings, such as the user's motion mode and the device mode, these methods suffer from robustness issues and might collapse if the underlying assumption is not satisfied. One solution to the problem is to classify the mode of the system and use the additional information obtained from this knowledge to robustify the algorithm.

IMU signals also contain what will be referred to as the gait signature that is caused by the steps we make when moving. Examples include bio-mechanical analysis of limping patterns for diagnosis of certain diseases such as Parkinson's [20]. An approach for computing a unique gait signature using measurements collected from IMU is proposed in Chapter 6.

The gait signature as observed by the IMU depends on both gait and device modes and as such reveals a rich information source suitable for a variety of applications. Our key contribution is a proposed algorithm for off-line analysis of IMU data during motion, with the following outline:

1. Gait segmentation using optimization to maximize similarity of the gait cycles. This step might need initialization, and here classical step detection algorithms can be used.

2. Estimation of the gait signature by averaging over the segments. This is done on a normalized time scale, so small variations in step cycle times are handled by resampling techniques.

3. Extraction of a low dimensional feature vector for the gait cycle using Fourier series analysis on the estimated gait signature. This feature vector includes physically explainable patterns.

Although the algorithm used for gait signature estimation is tailored for off-line applications, on-line extensions are also plausible. Thus, the gait signature estimation method can be used for either on-line classification, or off-line gait analysis. Figure 1.4 summarizes the gait segmentation approach introduced in Chapter 6. 


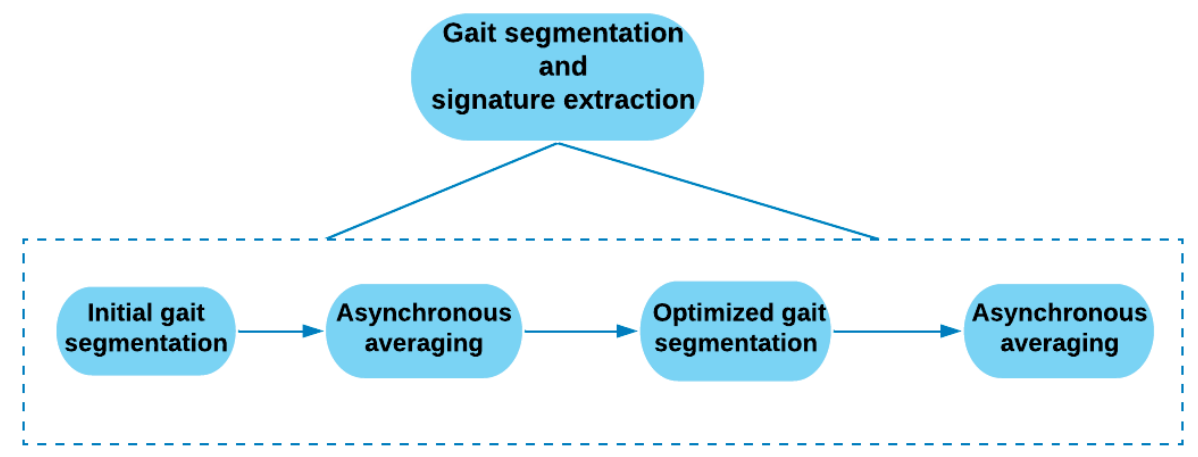

Figure 1.4: The flow diagram of the gait signature extraction using norm of pre-processed IMU signals.

\subsubsection{Joint Pedestrian Motion State and Device Pose Classification}

The joint classification problem, to the best of our knowledge, is rather an unexplored area with the exception of [102] in which machine learning approaches such as multilayer perceptron (MLP) and support vector machine (SVM) have been considered to identify the activity mode and device placement simultaneously. Various frequency and time domain features are extracted to create the model that fits the data. The classification rate is evaluated based on the joint device pose and the motion activities.

The main contribution of Chapter 7 is to shed a light on joint device mode and activity mode classification using standard machine learning methods and more advanced neural networks. The proposed method consists of four main building blocks as presented in Figure 1.5. The extracted IMU measurements are first pre-processed and then fed into the next block in which unique signatures for each gait mode and device mode are extracted using a linear search optimization algorithm [50].

The extracted signatures are then used to train classifiers for multi-class classification which completes the training phase of the proposed method. The performance at this stage is evaluated using the trained classifier applied to the development and test data. Finally, an additional HMM stage is applied on the coarse estimated classes. In this application, the hidden states in the HMM block are the activity modes and the device poses, i.e., the classes. The performance of the proposed classification techniques will be compared to the algorithm suggested in [102]. The classified gait cycles reveal which signature the current gait cycle belongs to. This additional information can further be used for gait cycle tuning. Consequently, step lengths can be estimated accurately, resulting in high precision gait cycles. The final result, for example, can be a more accurate PDR algorithm for pedestrian navigation purposes or activity analysis. 


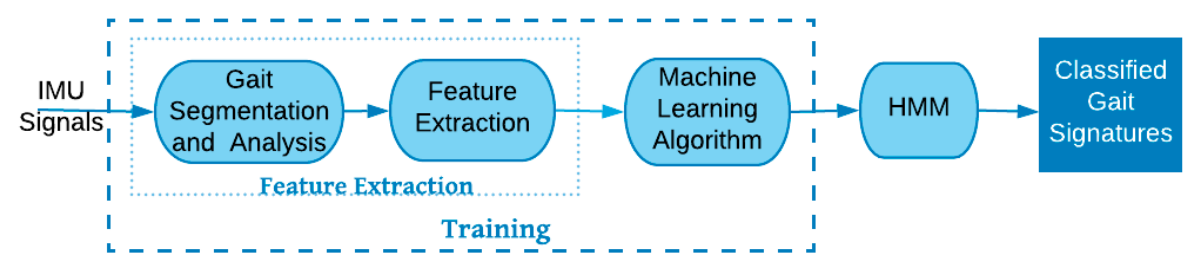

Figure 1.5: Overview of the classification process for joint activity and smartphone mode recognition.

\subsection{Contributions and Publications}

The main contributions of this thesis are listed below.

1. Development of an extension of a pedestrian dead reckoning model. Some parts of the contribution is published in the paper:

P. Kasebzadeh, C. Fritsche, G. Hendeby, F. Gunnarsson, F. Gustafsson. Improved Pedestrian Dead Reckoning Positioning With Gait Parameter Learning. In Proceedings of the 19th International Conference on Information Fusion, pages 379-385, Heidelberg, Germany, July 2016. (C) 2016 IEEE.

The main idea of this work originated from Fredrik Gustafsson and further refined and extended by discussions among all authors. Theoretical derivations, experimental results and writing the majority of the manuscript are by the author of this thesis. The co-authors aided in discussions and provided feedback to shape up the manuscript.

The content is reused in this thesis by courtesy of IEEE. This contribution corresponds to the material presented in Chapters 1,2 and 4. The models that are used for state estimation in this application are presented in Section 2.3. The material presented in this thesis is an extended version of the paper.

2. Classification of human motion activity modes and device modes. The contribution is published in the paper:

P. Kasebzadeh, G. Hendeby, C. Fritsche, F. Gunnarsson, F. Gustafsson. IMU Dataset For Motion and Device Mode Classification. In Proceedings of the 8th International Conference on Indoor positioning and indoor navigation, Sapporo, Japan, September 2017. C2017 IEEE.

The idea of this paper originated from Gustaf Hendeby and Fredrik Gustafsson. All the measurement experiments, dataset arrangements, 
theoretical derivations, experimental results and writing the majority of the manuscript are done by author of this thesis. The co-authors aided in discussions and feedback to shape up the manuscript in a good way.

The content is reused in this thesis by courtesy of IEEE. This contribution corresponds to the material presented in Chapter 1 and Chapter 5.

3. Optimizing the gait segmentation to maximize the similarity of the gait cycles. The contribution is submitted as the manuscript:

P. Kasebzadeh, G. Hendeby, and F. Gustafsson. Asynchronous averaging of gait cycles for classification of gait and device modes. Submitted to IEEE Sensors Journal, 2019a.

The main idea of this work originated from Fredrik Gustafsson and further refined and extended by discussions among the authors. Theoretical derivations, implementations of algorithms and experimental results are by the author of this thesis. The manuscript is written by the author of this thesis. This contribution corresponds to the material presented in Chapters 1, 2 and 6.

4. Joint gait mode and device mode classification using novel features. The contribution is submitted as the manuscript:

P. Kasebzadeh, K. Radnosrati, G. Hendeby, and F. Gustafsson. Joint pedestrian motion state and device pose classification. Submitted to IEEE Transactions on Instrumentation \& Measurement, 2019b.

The main idea of this work originated from the author of this thesis and further refined and extended by discussions among all authors. Experimental results and implementation of algorithms are by the author of this thesis. The manuscript is written by collaboration between the author of this thesis and Kamiar Radnosrati. This contribution corresponds to the material presented in Chapters 1, 3 and 7.

5. The first and second contribution have already been published in the author's licentiate's thesis:

P. Kasebzadeh. Parameter Estimation for Mobile Positioning Applications. Linköping Studies in Science and Technology. Licentiate Thesis. No. 1786, 2017.

Consequently, the contents of the two theses partially overlap.

An excluded contribution is the development and analysis of models for jointly antenna and propagation model parameters estimation. The contribution is published in the paper: 
P. Kasebzadeh, C. Fritsche, E. Özkan, F. Gunnarsson, F. Gustafsson. Joint Antenna and Propagation Model Parameter Estimation using RSS measurements. In Proceedings of the 18th International Conference on Information Fusion, pages 98-103, Washington D. C., USA, July 2015. C2015 IEEE.

This contribution is excluded in this work, however, it is presented in details in the author's licentiate thesis. [47].

\subsection{Thesis Outline}

This thesis is divided into two parts and founded upon theories formulated in estimation theory and machine learning. The first part is devoted to the theoretical background relevant to the applications which are introduced in the second part. These applications are based on the published papers and submitted manuscripts listed in Section 1.4.

In Chapter 2, we describe the relevant background information for PDR. A general odometry model for PDR is presented. Additionally, the supporting measurements used to increase accuracy and reliability of the system are introduced. Furthermore, the measurement model that relates the observed quantities to the desired state are provided. Moreover, all the sensors and receivers that are used as the measurement tools are described in this chapter. The state estimation methods, in which the main goal is to infer unknown states of a system from available measurements, are also presented in this chapter.

Chapter 3 describes relevant background information for the classification and deep learning algorithms. Representations of the considered machine learning algorithms are provided in this chapter. Finally, based on the definition of HMM provided in Chapter 2, an additional stage to the classifiers is introduced to capture the temporal correlation. It will be shown in later chapters that this will improve the classifier's performance in terms of classification accuracy.

Chapter 4 presents the first contribution introduced in Section 1.4. PNSs in devices equipped with inertial sensors and GPS are considered and an improved PDR algorithm that learns gait parameters in time intervals when GPS is available is proposed. A novel filtering approach is given that learns internal gait parameters in the PDR algorithm, such as the step length and the step detection threshold. The approach is based on a multi-rate Kalman filter bank that estimates the gait parameters when position measurements are available, which improves PDR in time intervals when the position is not available.

Chapter 5 is dedicated to the second contribution mentioned in Section 1.4. The main contribution is to publish one of the most extensive datasets build up upon several rounds of measurements. In this chapter, a preliminary study of the classification of human motion modes and device modes is also presented. The classifiers are trained by standard features, typically used in the literature, extracted from IMU sensory data.

Chapter 6 presents the third contribution introduced in Section 1.4. An optimization approach is presented for the gait segmentation problem in which the objective is to maximize the gait cycle similarity. Moreover, estimation of the gait 
signature by averaging over all the segments is performed. Finally, extraction of a low dimensional feature vector for the gait cycle using Fourier series analysis on the estimated gait signature is studied.

Chapter 7 is dedicated to the fourth contribution mentioned in Section 1.4. The problem of joint gait and device modes classification is presented in this chapter. Novel features have been extracted based on the results of the proposed gait segmentation method introduced in Chapter 6. The classifiers are trained and evaluated using different machine learning algorithms as well as a deep learning approach. ter 8 .

The work is summarized and a discussion of future work is presented in Chap- 

Part I

\section{Background}





\section{2}

\section{Pedestrian Dead Reckoning Positioning}

Position and orientation information can be obtained by integrating inertial sensor (accelerometer and gyroscope) measurements. The integration process is called Dead Reckoning (DR) in which the subject's known previous position, is processed to estimate the unknown current position. For example, the processing phase can be advancing the previously determined position over elapsed time based on known or estimated speed. The main advantage of DR is that it is independent of extra infrastructures and radio signals. This allows DR to be applied in many navigation applications such as pedestrian navigation and localization, marine navigation, automotive and autonomous navigation.

The price of being independent of extra infrastructures is the well-known disadvantage of all pure dead reckoning techniques; the errors that accumulate as time passes. This makes pure DR methods infeasible for most purposes while radio signal-based navigational systems provide highly accurate position information. For example, GPS-based navigation systems can provide position estimates with few meters accuracy. These methods, however, rely on the received signals and are prone to erroneous estimates in poor signal to noise ration (SNR) conditions or even failure in environments where the signal is blocked [4].

Nowadays, ubiquitous smartphones are embedded with inertial sensors such as accelerometer, gyroscope, and magnetometer. The sensors can be used for pedestrian navigation by means of dead reckoning. For navigational purposes, the accelerometer can be used as a pedometer to detect the steps and traveled distance and the built-in magnetometer as a compass heading provider. The gyroscope also provides information about how the phone is carried. PDR can be considered as an add-on to the other navigation methods or be used to extend the navigation techniques into areas where other systems are unavailable.

Fusing all pieces of information received from IMU sensors, it is possible to have a simple PDR implementation given that the subject holds an IMU unit in 
the hand in front of the body and walks with constant speed. The magnetometer measurements can be transformed into directional information and each step causes the position to move forward a fixed distance in the estimated direction. However, there are many challenges that must be considered in order to have more accurate estimates. The accuracy of the algorithm is limited by the sensor precision and magnetic disturbances. In addition to hardware and environmental factors, the algorithm design parameters need to be carefully tuned. For instance, how the algorithm detects a step from sensor measurements depends on a threshold that is set on the signal magnitude. How this threshold is defined together with other design considerations such as unknown gait parameters, like the length of each step, unknown phone position, the way that the phone is being carried, and user's motion mode identification are all among the challenges of PDR that are studied in detail in this thesis.

In Section 2.1, we describe all sensors which are used in this thesis for positioning and navigational purposes. Then, Section 2.2 presents general odometric models for PDR and introduces supporting measurements used to increase accuracy and reliability of the system. Finally, the hidden Markov model used for computing filtering and smoothing estimates of discrete states are presented in Section 2.4 .

\subsection{Sensors}

Depending on the application, different types of sensors can provide information for positioning. The sensed and reported quantity relates to the parameter or state of interest that is to be estimated. For example, in autonomous cars, sensors can be placed such that they provide information about the position and orientation of the car. Similarly, sensors can be used to obtain information about position and orientation of people that can further be applied to PDR algorithms.

Two kinds of sensors are commonly used in localization problems. One set of sensors measure those quantities that are indirectly related to the subject. The magnetometer is an example of this type measuring the surrounding magnetic field. The directional information can be inferred from the measurements. Other examples are cameras filming the surrounding, etc. The second set of sensors, measure values that are directly related to the subject. For example, the movement of the subject, orientation, etc. Inertial sensors for instance measure angular rate and acceleration that can be used for pose estimation.

\subsubsection{Inertial Measurements}

Significant developments of IMUs in recent years have enhanced their accuracy, made them smaller, and lowered their prices. This allows easy access to these units as either embedded in almost all recent smartphones or as dedicated devices. Figure 2.1 is an example of a sensor produced by Xsens which contains IMU sensors. We first describe the three main IMU sensors that are mainly used for positioning purposes. In this thesis, we use the accelerometer and gyroscope 


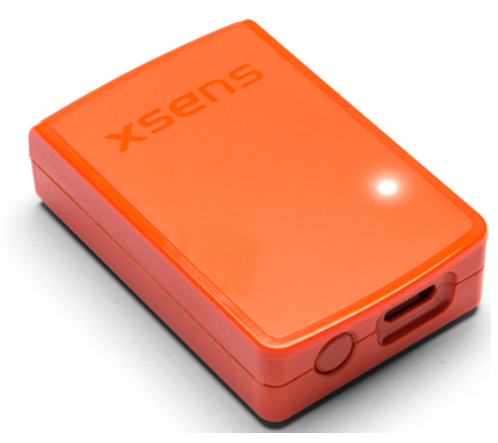

Figure 2.1: An Xsens motion sensor containing an IMU. Courtesy of Xsens Technologies.

sensors for estimating the PDR parameters and position estimation. In some applications, it might be necessary to use a magnetometer as a complementary sensor in addition to accelerometer and gyroscope [56].

\section{Accelerometer}

Accelerometers measure accelerations which can be used to estimate the change in position of the sensor. In accelerometers, the quantity that is measured is the specific force acting on the sensor. Both the earth's gravity and the sensor's acceleration contribute to the force measured by the sensor. However, the sensor's acceleration is generally much smaller in magnitude than the earth's gravity, $g=$ $9.81 \mathrm{~m} / \mathrm{s}^{2}$.

Gravity will, therefore, have a large contribution in the value provided by the accelerometer, while the motion of the sensor has a relatively small contribution. Subtracting the earth's gravity, the reported value is the non-gravitational force. The accelerometer measurement model is given as [93]

$$
y_{a, k}^{\mathrm{b}}=\mathbf{Q}_{k}^{b n}\left(a_{t}^{n}-\mathbf{g}^{n}\right)+b_{a, k}+e_{a, k},
$$

where $\mathbf{g}^{n}=[0,0, g]^{T}$ is the gravitation vector, $a_{t}^{n}$ is the sensor's acceleration in the navigation frame. $b_{a, k}$ denotes accelerometer bias, and $e_{a, k}$ is the accelerometer measurement noise. $\mathbf{Q}_{k}^{b n}$ is the rotation matrix in order to rotate the vector from navigation frame, $n$, which is a local geographic frame to body frame, $b$. The coordinate frame of the moving IMU with center in the accelerometer triad is called body frame. All the inertial measurements are resolved in this frame. More details about orientation representations and coordinate frames are presented in $[56]$. 


\section{Gyroscope}

Gyroscopes provide angular rates which can be used to define an estimate of the orientation of the sensor. The measured value is the angular velocity, i.e. rate of turn. Integrating the signal allows for adding up the changes in orientation over time. The gyroscope measurement model in body frame is given by [93]

$$
y_{g, k}^{b}=\omega_{z, k}^{b}+b_{g, k}+e_{g, k}
$$

where $\omega_{z, k}^{b}$ is the yaw rate which is the gyroscope measurement in the horizontal plane in body frame, $b$, with respect to the navigation frame. $b_{g, k}$ denotes gyroscope bias, and $e_{g, k}$.

The accuracy of measurements is a trade off between size and price of the sensor. For example, optical gyroscopes provide precise measurements but are hard to reduce much in size. MEMS, as introduced in the previous chapter, is an alternative technology aimed to create smaller devices.

\subsubsection{Supporting Measurements}

Supporting measurements could be obtained by additional sensors and/or receiver antennas that the mobile device is equipped with such as GPS. They are supposed to improve both the accuracy and reliability of the positioning algorithms. These supportive measurements, however, might not necessarily be synchronized with the IMU. Further, they might not always be available during the entire localization process. Therefore, they will be included in the measurement model whenever they are available.

The global positioning system is the most well-known global navigation satellite system (GNSS). The GPS receivers found in smartphones can provide direct measurements of the position, typically estimated from satellite pseudoranges, with few meters accuracy [34]. GPS receivers use multiple satellites and trilateration to determine the position and time of a user.

GPS satellites continuously transmit signals down to the earth over dedicated radio frequencies. The GPS receiver, among other data, receives a time stamp from the satellites momentary visible, along with satellite ephemeris data which contains the satellites' positions in the sky. The GPS receiver can then accurately calculate its position and time if it hears at least four satellites [64].

The GPS uses the World Geodetic System (WGS84) as its reference coordinate system [32]. By converting the Geodetic, WGS84, to Cartesian East-North-Up (ENU) coordinates, the estimated position can be either fused to the measurement model directly or be used to calculate the speed.

Accurate position (and time) estimation using GPS measurements, however, is not guaranteed when the received satellite signals are weak. This is a shortcoming of GNSS systems and can lead to poor position estimates or even a failure. 


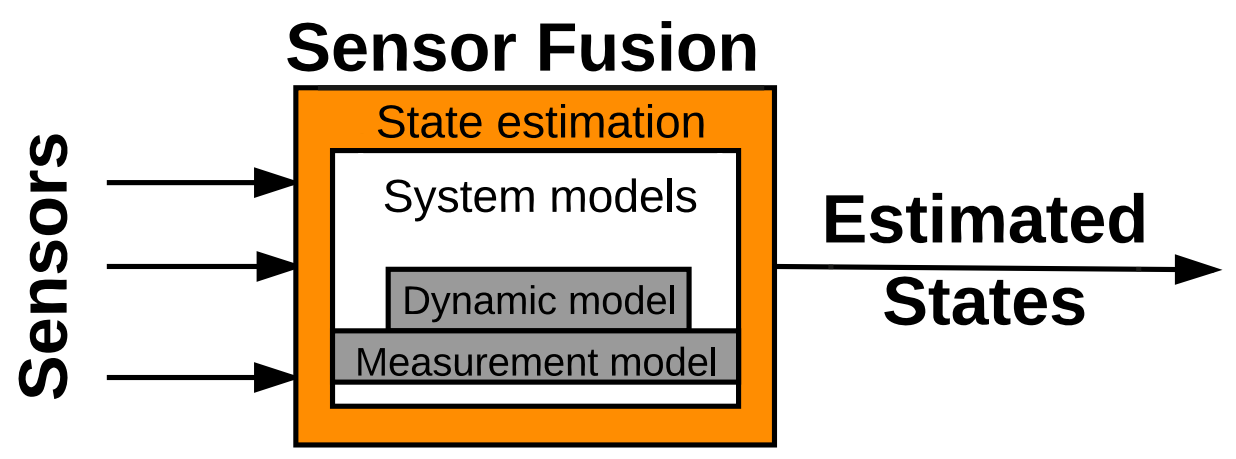

Figure 2.2: Illustration of a sensor fusion framework [19].

\subsubsection{Sensor Fusion}

Estimating the quantities of interest, $\mathbf{x}_{k}$, using some or all of the measurements $\mathbf{y}_{k}$ introduced above, is a sensor fusion problem. The process model, defining how $\mathbf{x}_{k}$ evolves over time, and measurement model, defining how $\mathbf{y}_{k}$ relates to $\mathbf{x}_{k}$, are used to infer the properties $\mathbf{x}_{k}$ from the measurements $\mathbf{y}_{k}$. Often, $\mathbf{x}_{k}$ is called state and represents the sought system property. States to be estimated can correspond to a wide variety of quantities from unknown constant properties such as weight to time varying position or orientation of a unit or even the surrounding environment. Orientation of the unit together with its position and velocity are the typical components of the states in this thesis.

One way to estimate each entity of the state vector $\mathbf{x}_{k}$ is to use information given by the sensors individually. Another alternative, used in the sensor fusion framework, is to fuse all the information obtained from inertial sensors, magnetometers, and GPS measurements and filter them to get the joint estimate of all entities of the states $\mathbf{x}_{k}$. The output of the filter is both the estimated states $\hat{\mathbf{x}}_{k}$ and a measure of how uncertain the estimates are.

Applying sensor fusion algorithms to obtain the joint estimate $\hat{\mathbf{x}}_{k}$ leads to performance improvements compared to the estimates obtained by each sensor individually. Evaluation of the performance is based on multiple criteria such as accuracy and robustness and number of sensors needed.

Fig 2.2 illustrates a generic overview of the fusion algorithm. For the framework to provide $\hat{\mathbf{x}}_{k}$, three components are needed; measurements $\mathbf{y}_{k}$ that relate to the system states $\mathbf{x}_{k}$ through measurement models, a model of the system dynamics, and a state estimation system that provides $\hat{\mathbf{x}}_{k}$. In this framework, rather than treating each sensor measurement individually, all measurements from all sensors, either of the same type or measurements of different types, are used as the input to the state estimation box. 


\subsection{Model Framework}

The generic state vector used in PDR problems consists of three components

$$
\mathbf{x}_{k}=\left(\begin{array}{l}
\mathrm{x}_{k} \\
\mathrm{y}_{k} \\
\psi_{k}
\end{array}\right),
$$

where $x_{k}$ and $y_{k}$ are the Cartesian positions and $\psi_{k}$ is the heading, see [37]. The input signals are given as

$$
\mathbf{u}_{k}=\left(\begin{array}{c}
v_{k} \\
\dot{\psi}_{k}
\end{array}\right),
$$

where $v_{k}$ is the velocity and $\dot{\psi}_{k}$ is the yaw rate. The dynamic model is described by

$$
\begin{aligned}
\mathrm{x}_{k+1} & =\mathrm{x}_{k}+T v_{k} \cos \left(\psi_{k}\right), \\
\mathrm{y}_{k+1} & =\mathrm{y}_{k}+T v_{k} \sin \left(\psi_{k}\right), \\
\psi_{k+1} & =\psi_{k}+T \dot{\psi_{k}},
\end{aligned}
$$

where $T$ is the sampling interval. These inertial signals are given by sensors either mounted on or being carried by the subject. Depending on the application, other components might be added to the state vector (2.3) such as sensor bias.

The measurements collected by both a tri-axial accelerometer and a tri-axial gyroscope sensors are used for position and PDR parameter estimation. As this figure suggests, the position of the sensor is estimated by double integration of the acceleration signal without the contribution of the earth's gravity. This requires subtracting the earth's gravity from the accelerometer measurements. Thus, to do the subtraction, the orientation of the device needs to be known a priori. Hence, when inertial sensors are used to estimate the position, the estimation of the orientation is the first step.

Subsequently, different supporting measurements such as GPS outdoor, angle dependent RSS outdoor, RSS fingerprint maps indoor, and proximity sensors are added to the measurement model to provide more accurate estimation. The state vector is also application dependent and the generic state (2.3) is extended by other components accordingly. For example, one might add the travelled distance, length of a taken step, threshold for step detection, etc. In the subsequent chapters, various problem formulations leading to an extended state vector will be described in more details.

\subsection{State-Space Estimation}

This section begins with a brief introduction to stochastic state-space models (SSMs). Prediction, filtering, and smoothing of the states are treated separately and explained in Section 2.3.2. The exact and optimal solution to state estimation problems under linearity and known Gaussian noise statistics assumptions 


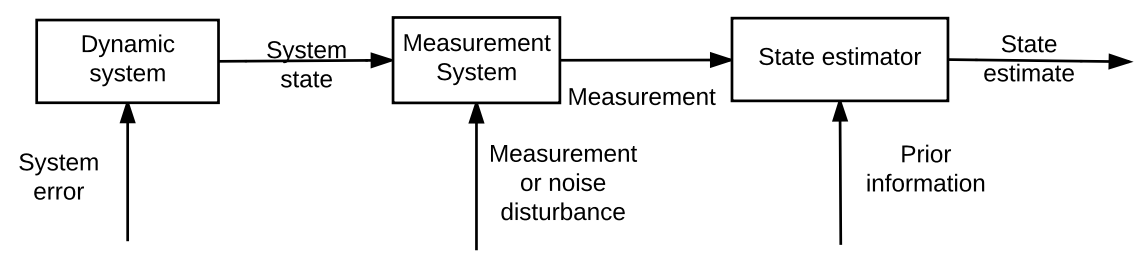

Figure 2.3: Block diagram of the state estimation problem [9].

is given by the Kalman filter (KF) as explained in Section 2.3.3. Section 2.3.4 relaxes the linearity assumption and provides algorithms for nonlinear SSM.

\subsubsection{Stochastic State Space Models}

State-space models relate the observed measurements to the latent state variable. The latent variables are not observed, but are reconstructible from the measured data. In the SSM framework, the measurement equation and system dynamics are modeled separately, where the latter predicts how the states of the system evolve over time.

The system dynamics are often continuous in time while observations are taken at discrete time instants. In this work, the system dynamics are restricted to discrete time descriptions. These are obtained by discretization of the continuous time dynamics where differential equations are replaced with difference equations.

To better model the characteristics of real systems, a method for predicting behaviors with some kind of randomness is required. Thus, a stochastic SSM is used to model a signal by introducing stochastic variables into the SSM [8]. The randomness can be modeled in both continuous and discrete times. However, stochastic variables in discrete time are studied in this work. An introduction to stochastic SSM is given in $[8,62]$.

\section{General Descriptions}

A generic form of stochastic SSM equations for the $k$-th time index can be given as

$$
\begin{aligned}
\mathbf{x}_{k+1} & =\mathbf{f}_{k}\left(\mathbf{x}_{k}, \mathbf{w}_{k}\right), \\
\mathbf{y}_{k} & =\mathbf{h}_{k}\left(\mathbf{x}_{k}, \mathbf{e}_{k}\right),
\end{aligned}
$$

where $\mathbf{x}_{k} \in \mathbb{R}^{n}$ is the current state vector, and $\mathbf{w}_{k} \in \mathbb{R}^{n_{\mathrm{w}}}$ is the unmeasured disturbance input to the system. $\mathbf{x}_{k+1}$ is the upcoming state, and $\mathbf{e}_{k} \in \mathbb{R}^{n_{e}}$ is the measurement noise and $\mathbf{y}_{k} \in \mathbb{R}^{m}$ contains measurements. The state difference (2.6a) uses a function $\mathbf{f}_{k}(\cdot)$, to account for the relation between the current state and the 
process noise with the upcoming state. The measurement equation (2.6b) utilizes a function $\mathbf{h}_{k}(\cdot)$ to connect the current state and the measurement noise $\mathbf{e}_{k}$ to $\mathbf{y}_{k}$.

The initial state $\mathbf{x}_{0}$, the noise signals $\mathbf{w}_{k}$ and $\mathbf{e}_{k}$ are the stochastic variables of the SSM. The probabilistic state-space model and the stochastic variables $\mathbf{w}_{k}$, and $\mathbf{e}_{k}$ are defined as

$$
\begin{aligned}
\mathbf{x}_{k} & \sim p\left(\mathbf{x}_{k} \mid \mathbf{x}_{k-1}\right), \\
\mathbf{y}_{k} & \sim p\left(\mathbf{y}_{k} \mid \mathbf{x}_{k}\right), \\
\mathbf{w}_{k} & \sim p\left(\mathbf{w}_{k}\right), \\
\mathbf{e}_{k} & \sim p\left(\mathbf{e}_{k}\right), \\
\mathbf{x}_{0} & \sim p\left(\mathbf{x}_{0}\right) .
\end{aligned}
$$

Generally, marginal probability densities are used as in (2.6) with complementary information about correlation between $\mathbf{x}_{0}, \mathbf{w}_{k}$, and $\mathbf{e}_{k}$. The notation in $(2.7 \mathrm{e})$ means that $\mathbf{x}_{0}$, for instance, has a distribution with density $p\left(\mathbf{x}_{0}\right)$.

Some assumptions are made for the problems considered in this work. First, time index starts at $k=0$ and the first measurement is taken at the next time, $k=1$. At each time instance $k$, both functions $\mathbf{f}_{k}(\cdot)$ and $\mathbf{h}_{k}(\cdot)$ are known. The noise, $\mathbf{w}_{k}$ and $\mathbf{e}_{k}$, characteristics as well as the distribution of $\mathbf{x}_{0}$ are also assumed to be known. For simplicity, the noises are assumed to be zero mean Gaussian independent random variables. Moreover, the initial state $\mathbf{x}_{0}$ is assumed to be independent to the measurement and process noises.

As stated earlier, (2.6) corresponds to a very generic SSM, spanning a broad range of features. For example, there is no restriction on functions $\mathbf{f}_{k}(\cdot)$ and $\mathbf{h}_{k}(\cdot)$ in terms of time-varying or time-invariant characteristics. Furthermore, nonlinearity in both functions can be of any kind. Stochastic variables can also follow any arbitrary distribution. However, a common special case of (2.6) is the stochastic SSM with nonlinear states in the dynamic model with a white Gaussian noise entering the model additively. It is called the additive white Gaussian noise state-space model

$$
\begin{aligned}
\mathbf{x}_{k+1} & =\mathbf{f}_{k}\left(\mathbf{x}_{k}\right)+\mathbf{w}_{k}, \\
\mathbf{y}_{k} & =\mathbf{h}_{k}\left(\mathbf{x}_{k}\right)+\mathbf{e}_{k},
\end{aligned}
$$

where transition function, $\mathbf{f}_{k}(\cdot)$, and measurement function, $\mathbf{h}_{k}(\cdot)$, are both arbitrary nonlinear functions. The dynamic and the measurement models of the system are represented by their corresponding PDFs as $p\left(\mathbf{x}_{k+1} \mid \mathbf{x}_{k}\right)$ and $p\left(\mathbf{y}_{k} \mid \mathbf{x}_{k}\right)$, respectively. The initial state is defined as

$$
\mathbf{x}_{0} \sim \mathcal{N}\left(\overline{\mathbf{x}}_{0}, \mathbf{P}_{0}\right),
$$

and the white Gaussian noise $\mathbf{w}_{k}$ and $\mathbf{e}_{k}$ are independent and with the following distributions

$$
\begin{aligned}
\mathbf{w}_{k} & \sim \mathcal{N}\left(0, \mathbf{Q}_{k}\right), \\
\mathbf{e}_{k} & \sim \mathcal{N}\left(0, \mathbf{R}_{k}\right) .
\end{aligned}
$$

The presented approximate filtering techniques in Section 2.3.4 employ (2.8). 


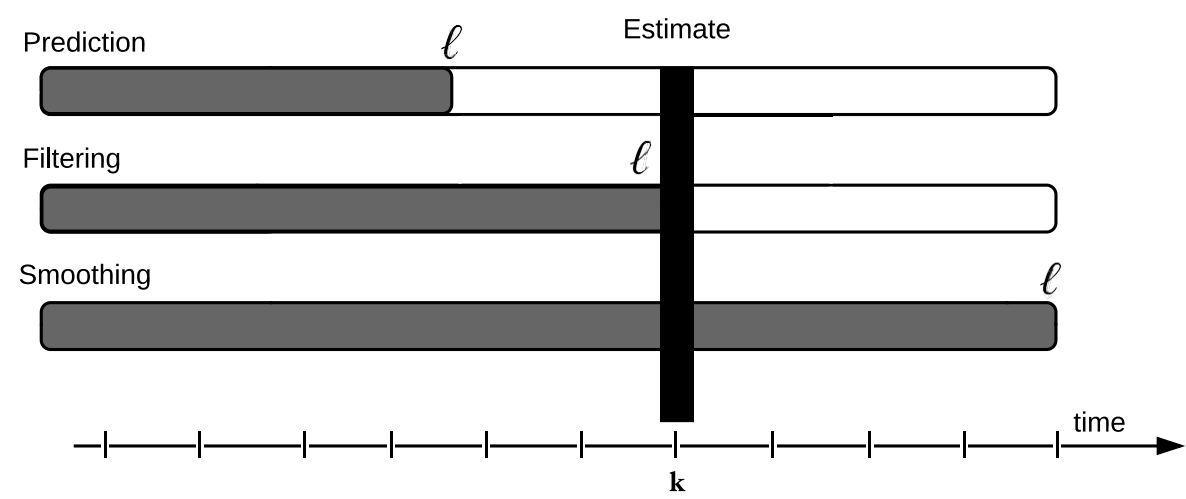

Figure 2.4: Illustration of different estimation problems. The state of interest is indicated with a black bar at time $k$. The available measurements up to time $\ell$ are illustrated by gray color [81].

\subsubsection{State Estimation}

Given a set of measurements $\mathbf{Y}_{1: \ell}=\left[\mathbf{y}_{1}, \ldots, \mathbf{y}_{\ell}\right]$, the estimation problem casts in to the one that infers the state estimates $\hat{\mathbf{X}}_{1: \ell}=\left[\hat{\mathbf{x}}_{1}, \ldots, \hat{\mathbf{x}}_{\ell}\right]$ of the true states $\mathbf{X}_{1: \ell}=\left[\mathbf{x}_{1}, \ldots, \mathbf{x}_{\ell}\right]$. The state-space model (2.12) is used for state estimation.

In the Bayesian framework, states are interpreted as random variables with a certain distribution. Given the prior knowledge of the stochastic processes $\mathbf{w}_{k}$, and $\mathbf{e}_{k}$, the objective of the Bayesian state estimation problem is to find the conditional posterior density $p\left(\mathbf{x}_{k} \mid \mathbf{Y}_{1: \ell}\right)$. The posterior estimation, given all measurements up to $\ell$, gradually becomes computationally intractable when $\ell$ gets large. To tackle this problem, Bayesian state estimation is solved under an additional assumptions to deal with this issue. That is, the state $\mathbf{x}_{k}$, follows a Markov process

$$
p\left(\mathbf{x}_{k} \mid \mathbf{x}_{0}, \ldots, \mathbf{x}_{k-1}\right)=p\left(\mathbf{x}_{k} \mid \mathbf{x}_{k-1}\right) .
$$

Nevertheless, the discussion of practical algorithms is postponed to the next section and here, we continue to describe the concepts behind the Bayesian state estimation problem.

Generally, the marginal distributions can be considered as a solution to state estimation problems instead of full join distributions which require complex computations. Filtering, prediction and smoothing are examples of these marginal distributions. These different distribution are distinguished by the temporal relation between the time, $k$, at which states are estimated and the available measurements time interval, $\ell$, as illustrated in Figure 2.4 and more thoroughly discussed in $[9,81]$. 
- Prediction

The prediction phase of the Bayesian filter computes the prediction distributions. The first row in Figure 2.4 illustrates a prediction scheme, in which $\ell<k$. More details are given in $[9,81]$.

- Filtering

In the filtering problem, $k$ is equal to $\ell$ and the posterior density distribution which is going to be estimated now takes the form $p\left(\mathbf{x}_{k} \mid \mathbf{Y}_{1: k}\right)$. This can be defined as an online sequential problem where the filtering density can be computed quickly by applying Bayes' rule to the prediction density.

The second row in Figure 2.4 illustrates the Bayesian filtering problem where $\ell=k$. The gray part represents the available measurement sets which reaches up to and includes time $k$.

- Smoothing

A Bayesian smoother computes smoothing distributions. The third row in Figure 2.4 illustrates Bayesian smoothing problem in which $\ell>k$. The smoothing distributions are the marginal distributions of state $\mathbf{x}_{k}$ given certain measurement interval, $\mathbf{Y}_{1: \ell}$.

As mentioned in the beginning of this section, the final goal is to find a point estimate of the states represented by $\hat{\mathbf{X}}_{1: \ell}$ together with some measure of uncertainty assigned to each estimate. One approach is to estimate the full posterior density, $p\left(\mathbf{x}_{k} \mid \mathbf{Y}_{1: k}\right)$, as introduced in the filtering above, and then define a point estimate and the uncertainty indicator from the statistics of the approximated distribution.

The Kalman filtering algorithm applies to linear Gaussian systems and computes the full posterior $\mathcal{N}\left(\mathbf{x}_{k} ; \hat{\mathbf{x}}_{k \mid k}, \mathbf{P}_{k \mid k}\right)$. This allows for extracting an estimate of the state $\hat{\mathbf{x}}_{k \mid k}$ and covariance $\mathbf{P}_{k \mid k}$. The reason is that a linear transformation of a Gaussian distribution is also a Gaussian whose sufficient statistics are the value of interest. However, as mentioned before, nonlinear and/or non-Gaussian models require approximations and cannot be handled by the Kalman filters. In case of nonlinear models, the extended Kalman filters can be applied.

\section{Linear model}

Imposing the linearity assumption on the dynamic and measurement models simplifies the computations considerably. The well-known linear Gaussian statespace model is obtained by assuming the functions $\mathbf{f}_{k}(x)$ and $\mathbf{h}_{k}(x)$ to be linear as

$$
\begin{aligned}
\mathbf{x}_{k+1} & =\mathbf{F}_{k} \mathbf{x}_{k}+\mathbf{G}_{k} \mathbf{w}_{k}, \\
\mathbf{y}_{k} & =\mathbf{H}_{k} \mathbf{x}_{k}+\mathbf{e}_{k},
\end{aligned}
$$

where $\mathbf{F}_{k}$ is a transition matrix and $\mathbf{H}_{k}$ is a measurement matrix. These two matrices are assumed to be independent of the state $\mathbf{x}_{k}$.

The inference in the linear model is significantly simplified. The reason is that a linear transformation of the Gaussian distributed initial state, does not 
change the posterior distribution. That is, all subsequent predictions and states will also be Gaussian distributed. In the special case of linear model (2.12) with additive Gaussian noise contributions, the well-known Kalman Filter, presented in Section 2.3.3, provides the optimal state estimates.

In cases of nonlinear state-space models, approximate filtering algorithms treat nonlinearities in different ways. Markov chain Monte Carlo approaches directly estimate the distribution of the states which undergo a nonlinear transformation. Another method is to first derive the approximate linearized equations and then treat them as a linear state-space model. The extended Kalman filter (EKF) uses this approach and is introduced in Section 2.3.4.

\subsubsection{Kalman Filter}

The Kalman filter is a very popular algorithm for estimating the state of linear systems. This filter is named after Rudolph E. Kalman, one of the primary developers of its theory who introduced the method in [45]. Kalman provided recursive formulas suited for time-varying linear filtering problems. More details about the KF history can be found in [44].

The KF is an optimal estimator in the sense that it minimizes the estimated error covariance given that some presumed conditions are met. Furthermore, as mentioned in [6], for linear systems with arbitrary noise, the KF is the best linear unbiased estimator (BLUE). In fact, $\mathrm{KF}$ is a linear filter which updates the mean and the covariance of the estimate to minimize the error of the estimated parameters or states. Both the measurements and the state of the linear system are perturbed by white Gaussian noise. In situations where the noise is not Gaussian, the KF is still the best linear unbiased estimator while nonlinear estimators may have better performance.

Being the optimal estimator together with good implementation characteristics makes the KF a celebrated solution. Furthermore, the KF is convenient for online real-time processing which further broadens its applicability. Unlike available snapshot solutions where each measurement is processed separately, the KF fuses multiple information to provide a better estimate. Using the system dynamics modeled by physical laws of motion, for example, known control inputs, and the possibility of fusing measurements from different types, make the KF a powerful tool in estimation applications.

The states of linear state-space systems described by (2.12) can be estimated by the KF. There are many alternative ways to represent the KF equations as described and derived in [6] and [44]. In this thesis, the KF is presented with alternating time update phase and the measurement update phase, where the dynamics of the system is handled and the measurements are fused in the estimate, respectively.

The KF prediction step based on the dynamic model given by (2.12) can be expressed as

$$
\begin{aligned}
\hat{\mathbf{x}}_{k \mid k-1} & =\mathbf{F}_{k} \hat{\mathbf{x}}_{k-1 \mid k-1}, \\
\mathbf{P}_{k \mid k-1} & =\mathbf{F}_{k} \mathbf{P}_{k-1 \mid k-1} \mathbf{F}_{k}^{\top}+\mathbf{G}_{k} \mathbf{Q}_{k} \mathbf{G}_{k}^{\top},
\end{aligned}
$$


where $\hat{\mathbf{x}}_{k \mid k-1}$ is the mean and $\mathbf{P}_{k \mid k-1}$ is the covariance matrix of the Gaussian prior. Note that the subscript $k \mid k-1$ indicates the current value of a quantity at time $k$ given its value at time $k-1$. This prediction gives the new prior distribution $\mathcal{N}\left(\hat{\mathbf{x}}_{k \mid k-1}, \mathbf{P}_{k \mid k-1}\right)$ and is computed from the previous Gaussian posterior $\mathcal{N}\left(\hat{\mathbf{x}}_{k-1 \mid k-1}, \mathbf{P}_{k-1 \mid k-1}\right)$.

In the measurement update phase the prior is updated whenever a measurement is available. This process can be shown to be

$$
\begin{aligned}
\hat{\mathbf{y}}_{k} & =\mathbf{H}_{k} \hat{\mathbf{x}}_{k \mid k-1}, \\
\varepsilon_{k} & =\mathbf{y}_{k}-\hat{\mathbf{y}}_{k}, \\
\mathbf{S}_{k} & =\mathbf{H}_{k} \mathbf{P}_{k \mid k-1} \mathbf{H}_{k}^{\top}+\mathbf{R}_{k}, \\
\mathbf{K}_{k} & =\mathbf{P}_{k \mid k-1} \mathbf{H}_{k}^{\top} \mathbf{S}_{k}^{-1}, \\
\hat{\mathbf{x}}_{k \mid k} & =\hat{\mathbf{x}}_{k \mid k-1}+\mathbf{K}_{k} \varepsilon_{k}, \\
\mathbf{P}_{k \mid k} & =\left(\mathbf{I}-\mathbf{K}_{k} \mathbf{H}_{k}\right) \mathbf{P}_{k \mid k-1},
\end{aligned}
$$

where $\varepsilon_{k}$ and $\boldsymbol{S}_{k}$ are called innovation/residual and innovation/residual covariance matrix, respectively. $\varepsilon_{k}$ denotes the difference between the predicted and the observed output, while $S_{k}$ represents the uncertainty of the predicted output. The $\mathbf{K}_{k}$ is the Kalman gain and is a factor of the correction. $\hat{\mathbf{x}}_{k \mid k}$ and $\mathbf{P}_{k \mid k}$ are the mean and the covariance matrix of the Gaussian posterior, respectively. I is an identity matrix. There are many ways to formulate the covariance update $(2.14 \mathrm{f})$, for instance, $\mathbf{P}_{k \mid k}$ can be represented as a sum of two positive definite symmetric matrices which is called Joseph's form

$$
\mathbf{P}_{k \mid k}=\left(\mathbf{I}-\mathbf{K}_{k} \mathbf{H}_{k}\right) \mathbf{P}_{k \mid k-1}\left(\mathbf{I}-\mathbf{K}_{k} \mathbf{H}_{k}\right)^{\top}+\mathbf{K}_{k} \mathbf{R}_{k} \mathbf{K}_{k}^{\top} .
$$

However, it requires more matrix manipulations compared to (2.14f). The KF algorithm starts with a time update and is initialized with

$$
\begin{aligned}
\hat{\mathbf{x}}_{0 \mid 0} & =\mathbf{x}_{0}, \\
\mathbf{P}_{0 \mid 0} & =\mathbf{P}_{0} .
\end{aligned}
$$

\subsubsection{Extended Kalman Filter}

Although the Kalman filter could provide an optimal solution to some state estimation problems, some limiting assumptions are applied in its derivation; the state space model is linear and the predicted state and the measurement are jointly Gaussian distributed.

The underlying assumptions used in the derivation of the KF recursions make it inapplicable to many real life problems, where either the dynamics or the measurement models, or both, are nonlinear. The reason is that the joint prediction density, $p\left(\mathbf{x}_{k}, \mathbf{y}_{k} \mid \mathbf{Y}_{1: k-1}\right)$, is not Gaussian for nonlinear systems even if the noise sources are Gaussian. In these situations, the problem could be treated with approximate filtering algorithms. EKF applied to nonlinear models is demonstrated and evaluated in early studies in $[42,82,87]$. 
EKF is an extension to the KF with the possibility of handling nonlinear models. It handles the nonlinearities in the models by approximating the nonlinear models with corresponding linear models. That is, the nonlinear equations are first linearized and then the approximate filtering densities are computed.

Linearization is performed using the first order Taylor series expansion at the current estimate in the EKF. Then, the standard KF can be used. However, this approach might yield poor performance if the nominal trajectory deviates from the true $\mathbf{X}_{1: k}$. To overcome this issue, the EKF uses the available information found and estimated by the filter as the linearization points, consecutively. In this method, the latest estimate is regarded as the best available information on the state and is used for linearization in the next iteration.

Using the first order Taylor expansion on the nonlinear SSM (2.8) and simplifying the expressions by assuming that the process noise is additive, (2.8a) and $(2.8 \mathrm{~b})$ can be used in the time and the measurement update phases, respectively. The prediction phase for the EKF algorithm is given as follows

$$
\begin{aligned}
\hat{\mathbf{x}}_{k \mid k-1} & =\mathbf{f}\left(\hat{\mathbf{x}}_{k-1 \mid k-1}\right), \\
\mathbf{P}_{k \mid k-1} & =\mathbf{f}^{\prime}\left(\hat{\mathbf{x}}_{k-1 \mid k-1}\right) \mathbf{P}_{k-1 \mid k-1} \mathbf{f}^{\prime}\left(\hat{\mathbf{x}}_{k-1 \mid k-1}\right)^{\top}+Q_{k},
\end{aligned}
$$

where $\mathbf{f}^{\prime}(\cdot)$ is the gradient of $\mathbf{f}(\cdot)$ with respect to $\mathbf{x}$ which is

$$
\mathbf{f}^{\prime}\left(\hat{\mathbf{x}}_{k-1 \mid k-1}\right)=\left.\frac{\partial}{\partial \mathbf{x}} \mathbf{f}(\mathbf{x})\right|_{\mathbf{x}=\hat{\mathbf{x}}_{k-1 \mid k-1}} .
$$

The new prior distribution, $\mathcal{N}\left(\hat{\mathbf{x}}_{k-1 \mid k}, \mathbf{P}_{k-1 \mid k}\right)$, is computed based on the previous Gaussian posterior $\mathcal{N}\left(\hat{\mathbf{x}}_{k-1 \mid k-1}, \mathbf{P}_{k-1 \mid k-1}\right)$. The next step is the measurement update which updates the prior with the new information whenever new measurements are available

$$
\begin{aligned}
\hat{\mathbf{y}}_{k} & =\mathbf{h}\left(\hat{\mathbf{x}}_{k \mid k-1}\right), \\
\varepsilon_{k} & =\mathbf{y}_{k}-\hat{\mathbf{y}}_{k}, \\
\mathbf{S}_{k} & =\mathbf{h}^{\prime}\left(\hat{\mathbf{x}}_{k \mid k-1}\right) \mathbf{P}_{k \mid k-1} \mathbf{h}^{\prime}\left(\hat{\mathbf{x}}_{k \mid k-1}\right)^{\top}+\mathbf{R}_{k}, \\
\mathbf{K}_{k} & =\mathbf{P}_{k \mid k-1} \mathbf{h}^{\prime}\left(\hat{\mathbf{x}}_{k \mid k-1}\right)^{\top} \mathbf{S}_{k}^{-1}, \\
\hat{\mathbf{x}}_{k \mid k} & =\hat{\mathbf{x}}_{k \mid k-1}+\mathbf{K}_{k} \boldsymbol{\varepsilon}_{k}, \\
\mathbf{P}_{k \mid k} & =\left(\mathbf{I}-\mathbf{K}_{k} \mathbf{h}^{\prime}\left(\hat{\mathbf{x}}_{k \mid k-1}\right)\right) \mathbf{P}_{k \mid k-1},
\end{aligned}
$$

where $\mathbf{h}^{\prime}(\cdot)$ is the Jacobian of the $\mathbf{h}(\cdot)$ function

$$
\mathbf{h}^{\prime}\left(\hat{\mathbf{x}}_{k \mid k-1}\right)=\left.\frac{\partial}{\partial \mathbf{x}} \mathbf{h}(\mathbf{x})\right|_{\mathbf{x}=\hat{\mathbf{x}}_{k \mid k-1}} .
$$

The EKF algorithm starts with performing the time update phase and assigning the initial value for the states and the covariance matrix similar to the KF. That is, $\hat{\mathbf{x}}_{0 \mid 0}=\mathbf{x}_{0}$ and $\mathbf{P}_{0 \mid 0}=\mathbf{P}_{0}$. As discussed earlier, the linearization in the measurement update at time $k$ is based on the predicted state $\hat{\mathbf{x}}_{k \mid k-1}$.

The second order EKF, also known as EKF with bias compensation, is one example where the Hessian of the nonlinear equation is computed at each iteration 
to achieve a better approximation. One shortcoming of such algorithms is the necessity of higher order differentiations that can cause vastly increased computational complexity.

\subsubsection{Rauch-Tung-Striebel Smoother}

At each time step $k$, Bayesian smoothers can estimate the posterior distribution of the state conditioned on all obtained measurements, $p\left(\mathbf{x}_{k} \mid \mathbf{Y}_{1: \ell}\right)$. In other words, given all the measurements in $\{1: \ell\}$, Bayesian smoothing, at time step $k$, computes the marginal posterior distribution of the state $x_{k \mid \ell}$ where $\ell>k$.

For computing the marginal posterior distribution, as the first step, filtering posterior distributions of the states should be obtained by a normal Bayesian filter, followed by a backwards recursion [54].

The Rauch-Tung-Striebel smoother (RTSS) provides the closed form smoothing solution to the linear filtering model $(2.12)[17,75,81]$

$$
p\left(\mathbf{x}_{k} \mid \mathbf{Y}_{1: \ell}\right)=\mathcal{N}\left(\mathbf{x}_{k} \mid \hat{\mathbf{x}}_{k-1 \mid k}, \mathbf{P}_{k-1 \mid k}\right) \text {. }
$$

The RTSS performs a forward and backward recursion. The forward recursion is the same as the KF algorithm and the backward recursion for RTSS is

$$
\begin{aligned}
\mathbf{D}_{k-1 \mid k} & =\mathbf{P}_{k-1 \mid k-1} \mathbf{F}_{k}^{\top} \mathbf{P}_{k \mid k-1}^{-1}, \\
\hat{\mathbf{x}}_{k-1 \mid k} & =\hat{\mathbf{x}}_{k-1 \mid k-1}+\mathbf{D}_{k-1 \mid k}\left(\hat{\mathbf{x}}_{k \mid K}-\hat{\mathbf{x}}_{k \mid k-1}\right), \\
\mathbf{P}_{k-1 \mid k} & =\mathbf{P}_{k-1 \mid k-1}+\mathbf{D}_{k-1 \mid k}\left(\mathbf{P}_{k \mid K}-\mathbf{P}_{k \mid k-1}\right) \mathbf{D}_{k}^{\top},
\end{aligned}
$$

where $\mathbf{P}_{k \mid k-1}$ and $\mathbf{P}_{k-1 \mid k-1}$ are the covariances obtained by KF prediction step (2.13b) and measurement update phase (2.14f), receptively. $\hat{\mathbf{x}}_{k \mid k-1}$ and $\hat{\mathbf{x}}_{k-1 \mid k-1}$ are the mean computed by KF prediction step (2.13a) and KF measurement update $(2.14 \mathrm{e})$. The last time step $\ell$ is the starting point for the smoother recursion [81].

\subsubsection{Extended Rauch-Tung-Striebel Smoother}

Similar to the KF, RTSS can only be applied to linear models. Hence, for problems that have nonliner dynamic or/and measurement models RTSS is not applicable. Applying first order approximations to RTSS prediction equations is a solution for such problems which is called Extended Rauch-Tung-Striebel Smoother (ERTSS).

The ERTSS algorithm is defined as

$$
\begin{aligned}
\mathbf{D}_{k-1 \mid k} & =\mathbf{P}_{k-1 \mid k-1} \mathbf{f}^{\prime}\left(\hat{\mathbf{x}}_{k-1 \mid k-1}\right)^{\top} \mathbf{P}_{k \mid k-1}^{-1}, \\
\hat{\mathbf{x}}_{k-1 \mid k} & =\hat{\mathbf{x}}_{k-1 \mid k-1}+\mathbf{D}_{k-1 \mid k}\left(\hat{\mathbf{x}}_{k \mid K}-\hat{\mathbf{x}}_{k \mid k-1}\right), \\
\mathbf{P}_{k-1 \mid k} & =\mathbf{P}_{k-1 \mid k-1}+\mathbf{D}_{k-1 \mid k}\left(\mathbf{P}_{k \mid K}-\mathbf{P}_{k \mid k-1}\right) \mathbf{D}_{k}^{\top},
\end{aligned}
$$

where $\mathbf{P}_{k \mid k-1}$ and $\mathbf{P}_{k-1 \mid k-1}$ are the covariances obtained by the EKF prediction step (2.17b) and measurement update phase (2.19f), receptively. $\hat{\mathbf{x}}_{k \mid k-1}$ and $\hat{\mathbf{x}}_{k-1 \mid k-1}$ are the mean computed in the EKF prediction step (2.17a) and the EKF measurement update $(2.19 \mathrm{e})$. The matrix $\mathbf{f}^{\prime}(\cdot)$ is the Jacobian matrix of $f(\cdot)$. 


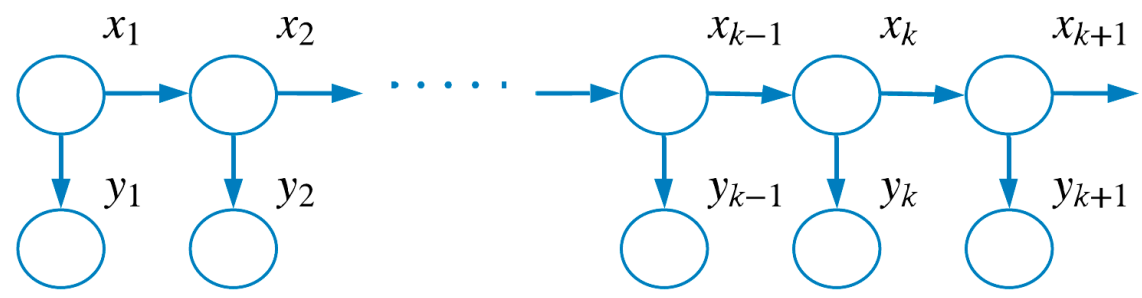

Figure 2.5: A Bayesian network representing the factorization of joint probability in the hidden Markov model. The sequential observations $y_{k}$ are conditioned on the corresponding state $x_{k}[14]$.

\subsection{Hidden Markov Model}

In this section, we deal with a discrete finite scalar state and present a well-known algorithm to find the filtering and smoothing distributions. The dynamic systems introduced in the previous section, in the contrary, solved the state-space problem with real-valued state vector. Hidden Markov model is one important category of state space models with applications in different areas such as artificial intelligence, pattern recognition, and computer vision [33]. HMM assumes that the observation at time $k, \mathbf{y}_{k}$, is generated by a process whose state, $\mathbf{x}_{k}$, is hidden from the observer and is discrete.

The graphical structure of the HMM is presented in Figure 2.5 in which, using the Bayesian networks properties, the conditional independence requirement of the discrete state is satisfied

$$
\mathbf{x}_{k+1} \Perp \mathbf{x}_{k-1} \mid \mathbf{x}_{k} .
$$

The joint distribution is then given by

$$
\begin{aligned}
p\left(\mathbf{x}_{1}, \ldots, \mathbf{x}_{K}, \mathbf{y}_{1}, \ldots, \mathbf{y}_{K}\right) & =p\left(\mathbf{x}_{1: K}\right) p\left(\mathbf{y}_{1: K} \mid \mathbf{x}_{1: K}\right) \\
& =p\left(\mathbf{x}_{1}\right)\left[\prod_{k=2}^{K} p\left(\mathbf{x}_{k} \mid \mathbf{x}_{k-1}\right)\right] \prod_{k=1}^{K} p\left(\mathbf{y}_{k} \mid \mathbf{x}_{k}\right),
\end{aligned}
$$

where the notation $\mathbf{x}_{1: K}$ denotes $\mathbf{x}_{1}, \ldots, \mathbf{x}_{K}$. Let $n_{x}$ denote the dimension of the discrete state vector. The marginal distribution over the initial state, $p\left(\mathbf{x}_{1}\right)$ is represented by a vector $\pi$.

Given the initial state distribution, in order to form the joint distribution (2.23), we need to define the transition model $p\left(\mathbf{x}_{k} \mid \mathbf{x}_{k-1}\right)$ and the observation model $p\left(\mathbf{y}_{k} \mid \mathbf{x}_{k}\right)$. In HMM, it is usually assumed that the transition and observation models are time-invariant [33]. Hence, the transition model can be defined by a $n_{x} \times n_{x}$ state transition matrix $\pi$ the elements of which are the transition probabilities. In this thesis, the observations used in HMM are discrete 
vectors of size $n_{y}$. Therefore, the observation model can be defined using a $n_{x} \times n_{y}$ emission matrix.

An efficient way of computing the posterior distribution of the states given the observations is by employing the forward-backward (FB) algorithm [72] or the Baum-Welch algorithm [12]. Among different variations of the same algorithm, the one known as alpha-beta algorithm $[14,73]$ is used in this thesis.

Using the FB algorithm, the filtering, $p\left(\mathbf{x}_{k} \mid \mathbf{y}_{1: k}\right)$, and smoothing, $p\left(\mathbf{x}_{k} \mid \mathbf{y}_{1: K}\right)$, distributions can be computed separately by defining two independent values

$$
\alpha\left(\mathbf{x}_{k}\right)=p\left(\mathbf{y}_{1}, \ldots, \mathbf{y}_{k}, \mathbf{x}_{k}\right)
$$

and

$$
\beta\left(\mathbf{x}_{k}\right)=p\left(\mathbf{y}_{k+1}, \ldots \mathbf{y}_{K} \mid \mathbf{x}_{k}\right) .
$$

For the considered discrete-time, first-order HMM, in which the hidden state $\mathbf{x}_{k}$ depends only on its one step predecessor, the selected distributions can be computed by

- Filtering:

$$
p\left(\mathbf{x}_{k} \mid \mathbf{y}_{1: k}\right)=\frac{\alpha\left(\mathbf{x}_{k}\right)}{\sum_{x_{k}} \alpha\left(x_{k}\right)},
$$

where it can be shown that the forward recursions for computing $\alpha\left(\mathbf{x}_{k}\right)$ are given by

$$
\alpha\left(\mathbf{x}_{k}\right)=p\left(\mathbf{y}_{k} \mid \mathbf{x}_{k}\right) \sum_{\mathbf{x}_{k-1}} \alpha\left(\mathbf{x}_{k-1}\right) p\left(\mathbf{x}_{k} \mid \mathbf{x}_{k-1}\right),
$$

initialized at $\alpha\left(\mathbf{x}_{1}\right)=p\left(\mathbf{y}_{1}, \mathbf{x}_{1}\right)=p\left(\mathbf{x}_{1}\right) p\left(\mathbf{y}_{1} \mid \mathbf{x}_{1}\right)$.

- Smoothing:

$$
p\left(\mathbf{x}_{k} \mid \mathbf{y}_{1: K}\right)=\frac{\alpha\left(\mathbf{x}_{k}\right) \beta\left(\mathbf{x}_{k}\right)}{\sum_{x_{k}} \alpha\left(x_{k}\right) \beta\left(x_{k}\right)},
$$

where it can be shown that the backward recursions for computing $\beta\left(\mathbf{x}_{k}\right)$ are given by

$$
\beta\left(\mathbf{x}_{k}\right)=\sum_{\mathbf{x}_{k+1}} \beta\left(\mathbf{x}_{k+1}\right) p\left(\mathbf{y}_{k+1} \mid \mathbf{x}_{k+1}\right) p\left(\mathbf{x}_{k+1} \mid \mathbf{x}_{k}\right),
$$

initialized at $\beta\left(\mathbf{x}_{K}\right)=1$.

\subsection{Summary}

This chapter has introduced models for sensor observations and general state space models, including a simple PDR model, and the most important tools (Kalman and HMM filters and smoothers) for making state inference applicable to both the PDR model, presented in Chapter 4, and other state space models, presented in Chapter 3. 


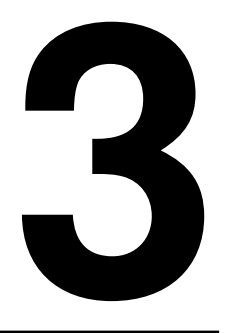

\section{Statistical Machine Learning}

Machine learning, in general terms, is the science of making machines learn, from past experiences, how to act in future without being explicitly programmed. Given a dataset containing different examples, the machine learning problem is to develop algorithms that learn from the examples to predict future, unseen, events.

Figure 3.1 presents a graphical perspective of the whole process in which the training data is given as the input. The machine then analyzes the data to find a meaningful pattern between different features of the given input and produces a statistical model of the data. The model is further used for different purposes, e.g. prediction, classification, etc.

In this chapter, basic concepts of machine learning problems are first presented in Section 3.1. Considering the main focus of this thesis, some classification methods are selected and summarized in Section 3.2. A feed forward neural network classifier, a deep learning approach, is presented in detail in Section 3.2.3. Section 3.3 employs the HMM framework introduced in Chapter 2 to capture the correlation in the considered classification problem.

\subsection{Basic Concepts}

The majority of practical machine learning falls into two main categories; supervised learning and unsupervised learning. Let $\mathcal{T}$ denote the training set containing $n_{r}$ training inputs, $\mathcal{T}=\left\{x^{r}\right\}_{r}^{n_{r}}$. In unsupervised learning, the goal is to learn more about the data by modeling its underlying distribution or structure.

In supervised learning problems, on the other hand, we have a labeled training set, denoted by $\mathcal{T}=\left\{\left(x^{r}, y^{r}\right)\right\}_{r}^{n_{r}}$, where $x$ and $y$ are the input-output pairs, respectively. The training input $x^{r}$, also known as feature, attribute or covariate, is normally a $D$-dimensional vector of simple or complex structured objects, such 


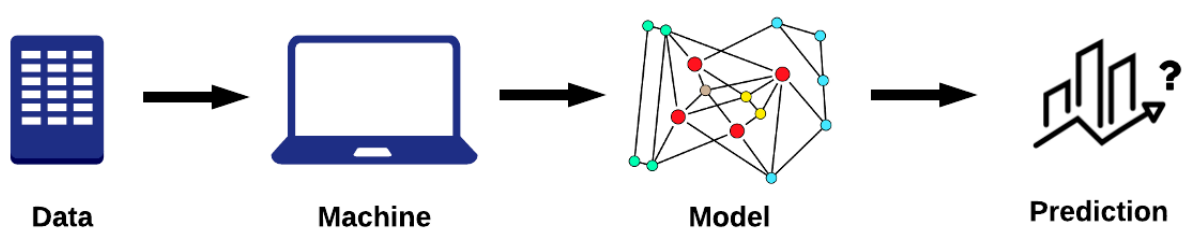

Figure 3.1: A schematic illustration of machine learning concept.

as number, image, graph, etc.

Supervised learning strives to learn a mapping from inputs to outputs that can be formulated as a function approximation problem. For any input $x$, output $y$ and an unknown function $q(\cdot)$, assume $y=q(x)$. The goal of learning is to approximate the true function $q$ given a labeled training set. This allows us to predict the output quantity for any, unseen, input after the training phase.

The most trivial solution for finding the output, upon receiving a new input, is to look through the training set and try to match the new input with the closest training example. The known output of the best match in the training example can then be set as the output of the unknown new input. To deal with most of practical problems, however, more complex algorithms are required.

The existing supervised learning algorithms are either parametric or nonparametric. In the former group, the data is assumed to follow a certain set of properties hence can be modeled using a fixed number of parameters. Nonparametric algorithms, on the other hand, make fewer assumptions about the data and use a flexible-number of parameters for modeling which typically grows throughout the learning process.

Parametric algorithms can be more computationally efficient than the nonparametric ones. However, if the data does not satisfy the underlying assumptions, performance degradation can be non-negligible. Therefore, there are computational cost and accuracy trade-offs between the two type of algorithms.

The output, response variable, can be a real-valued scalar as in regression problems or a finite set of nominal or categorical variables $y^{r}$ as in some classification applications. Problems with only two class labels, eg. $y^{r} \in 0,1$, are known as binary classification whereas multi-label classification, that is the scope of this thesis, refers to those problems in which $n_{c}>2$.

\subsection{Classification Methods}

In this work, we divide the data into training set of size $n_{r}$, and development set and test set, both of equal sizes $n_{d}=n_{e}=\frac{M-n_{r}}{2}$. It must be noted that the development and test set partitioning can be done in different ways. Here, given the size of the used dataset, we consider them to be of equal sizes.

It is worth noting that the development set is used to cross-validate all the trained algorithms using classifiers introduced in Section 3.2 in order to avoid overfitting, underfitting or any other penalties that might increase classification 
error of the test set. The hyperparameters of the algorithms are all selected such that the development set error is minimized. Then, as the final step the trained classifiers are applied to test set (the part of the dataset which has not been seen during the training procedure) and the performance of the proposed algorithms is evaluated and reported in Chapter 7.

\subsubsection{Weighted $K$-Nearest Neighbor}

The $K$-nearest neighbor (KNN) is a simple, non-parametric classifier which computes the distance, e.g. Euclidean distance, between the test (unseen) input and the training data points. Selecting the $K$ training points with the closest distance to the input, the algorithm counts how many members of each class are in this set.

Let $\boldsymbol{X}_{r}$ be the $r$ :th training example in the whole training set $\mathcal{T}$. For a new point in the development set, $\boldsymbol{X}_{d}$, the KNN classifier estimates the membership probability as the empirical fraction given by

$$
\begin{aligned}
\hat{\mathcal{C}}_{d}= & p\left(\boldsymbol{X}_{d} \in c \mid \mathcal{T}, K\right) \\
= & \frac{1}{K} \sum_{r \in N_{K}\left(\boldsymbol{X}_{d}, \mathcal{T}\right)} \mathbb{I}\left(\boldsymbol{X}_{r} \in c\right),
\end{aligned}
$$

where $N_{K}\left(\boldsymbol{X}_{d}, \mathcal{T}\right)$ are the indices of the $K$ nearest points to $\boldsymbol{X}_{d}$ in the training set $\mathcal{T} . \mathbb{I}(\cdot)$ is the indicator function defined as

$$
\mathbb{I}(v)= \begin{cases}1 & \text { if } v \text { is true } \\ 0 & \text { otherwise }\end{cases}
$$

The weighted-KNN is an extension of the KNN which gives higher weights to the classes in the training set which are particularly close to the new input $\boldsymbol{X}_{d}$ compared to the rest of the neighbors further away from $\boldsymbol{X}_{d}$. Let $w_{d, r}$ denote the weight assigned to each training example $r$, the membership probability can be computed as

$$
\hat{\mathcal{C}}_{d}=\frac{\sum_{r \in N_{K}\left(\boldsymbol{X}_{d}, \mathcal{T}\right)} w_{d, r} \times \mathbb{I}\left(\boldsymbol{X}_{r} \in c\right)}{\sum_{r \in N_{K}}\left(\boldsymbol{X}_{d}, \mathcal{T}\right)} w_{d, r} .
$$

The weighting scheme used in this thesis is the squared inverse Euclidean distance which gives each neighbor a weight of $w_{d, r}=1 / D_{r}{ }^{2}$, where $D_{r}$ is the Euclidean distance to the $r$ :th neighbor. 


\subsubsection{Gaussian Process}

A random process $q(\cdot)$ is Gaussian if

$$
\begin{aligned}
& \forall n \in \mathbb{N}, \quad \forall \delta_{1}, \ldots, \delta_{n} \in \mathbb{R}^{n}: \\
& \left(\begin{array}{c}
q\left(\delta_{1}\right) \\
q\left(\delta_{2}\right) \\
\vdots \\
q\left(\delta_{n}\right)
\end{array}\right) \sim \mathcal{N}\left(\left(\begin{array}{c}
\mu\left(\delta_{1}\right) \\
\mu\left(\delta_{2}\right) \\
\vdots \\
\mu\left(\delta_{n}\right)
\end{array}\right),\left(\begin{array}{ccc}
\kappa\left(\delta_{1}, \delta_{1}\right) & \ldots & \kappa\left(\delta_{1}, \delta_{n}\right) \\
\kappa\left(\delta_{2}, \delta_{1}\right) & \ldots & \kappa\left(\delta_{2}, \delta_{n}\right) \\
\vdots & \ddots & \vdots \\
\kappa\left(\delta_{n}, \delta_{1}\right) & \ldots & \kappa\left(\delta_{n}, \delta_{n}\right)
\end{array}\right)\right),
\end{aligned}
$$

where for two random variables $\delta$ and $\delta^{\prime}, \mu(\delta)=E[q(\delta)]$ is the mean function, and $\kappa\left(\delta, \delta^{\prime}\right)=E\left[(q(\delta)-\mu(\delta))\left(q\left(\delta^{\prime}\right)-\mu\left(\delta^{\prime}\right)\right)\right]$ is the positive definite covariance function. Let $\delta=\left[\delta_{1}, \ldots, \delta_{n}\right] \in \mathbb{R}^{n}$, we get

$$
q(\delta) \sim \mathcal{G P}\left(\mu(\delta), \kappa\left(\delta, \delta^{\prime}\right)\right),
$$

where the notation $\mathcal{G P}(\cdot, \cdot)$ denotes a Gaussian process (GP). Given the training set $\mathcal{T}$ containing input vectors and their corresponding class labels, $\left\{\boldsymbol{X}_{r}, c_{r}\right\}_{r=1}^{n_{r}}$, the Gaussian process classifier predicts the class membership probability for a new, unseen development point $\boldsymbol{X}_{d}$. If the function values $q^{(r)} \triangleq q\left(\boldsymbol{X}_{r}\right)$ were known, it would be possible to use a GP for regression to find $q\left(\boldsymbol{X}_{d}\right)$ and then map it into the interval $[0,1]$ by means of the softmax function. However, the training data only includes the class labels $c_{r}$.

To solve this problem, as discussed in [74], we introduce the latent function values at all $n_{r}$ training examples, for all classes

$$
q^{(r)} \triangleq \boldsymbol{q}=\left(q_{1}^{(1)}, \ldots, q_{1}^{\left(n_{r}\right)}, q_{2}^{(1)}, \ldots, q_{2}^{\left(n_{r}\right)}, \ldots, q_{n_{c}}^{(1)}, \ldots, q_{n_{c}}^{\left(n_{r}\right)}\right)^{\top}, \quad r=1, \ldots, n_{r} .
$$

Define $\mathcal{C}$ as a vector of the same length as $\boldsymbol{q}$. For each training example, the value of $\mathcal{C}$ for the true class label is 1 and for the rest of the classes is 0 . The distribution of the latent variable $q_{d}$, corresponding to the development point $\boldsymbol{X}_{d}$, can then be computed by

$$
p\left(q_{d} \mid \boldsymbol{X}_{d}, \mathcal{T}, \mathcal{C}\right)=\int p\left(q_{d}, \boldsymbol{q} \mid \boldsymbol{X}_{d}, \mathcal{T}, \boldsymbol{q}\right)=\int p\left(q_{d} \mid \boldsymbol{X}_{d}, \mathcal{T}, \boldsymbol{q}\right) p(\boldsymbol{q} \mid \mathcal{T}, \mathcal{C}) d \boldsymbol{q},
$$

which is further used to compute the class membership distribution

$$
\hat{\mathcal{C}}_{d}=\int \sigma\left(q_{d}\right) p\left(q_{d} \mid \boldsymbol{X}_{d}, \mathcal{T}, \mathcal{C}\right) d q_{d}
$$

where $\sigma\left(q_{d}\right)$ is the softmax function, which for any vector $\varphi \in \mathbb{R}^{n_{\varphi}, 1}$ is given by

$$
\sigma(\varphi)_{j}=\frac{\exp \left(\varphi_{j}\right)}{\sum_{i=1}^{n_{\varphi}} \exp \left(\varphi_{i}\right)}
$$

The non-Gaussian likelihood in (3.7) makes the integral analytically intractable. One approach is to use Laplace approximation [74, 97] to form 


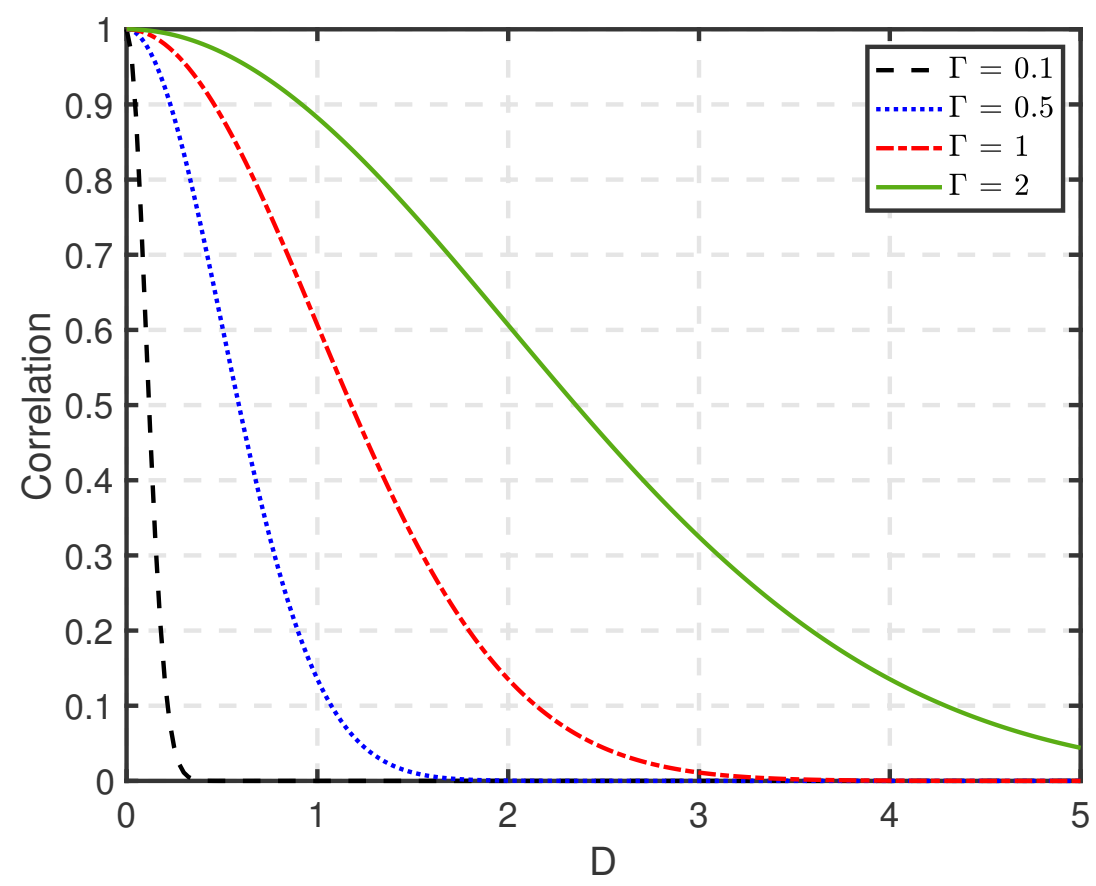

Figure 3.2: Squared exponential kernel for $\sigma_{q}=1$ and different values of $\Gamma$. Larger $\Gamma$ results in highly correlated values for close points.

$p(\hat{\boldsymbol{q}} \mid \mathcal{T}, \mathcal{C}) \approx \mathcal{N}\left(\hat{\boldsymbol{q}}, \boldsymbol{A}^{-1}\right)$ with the statistics estimated as

$$
\begin{aligned}
\hat{\boldsymbol{q}} & =\underset{\boldsymbol{q}}{\arg \max } p(\boldsymbol{q} \mid \mathcal{T}, \mathcal{C}), \\
\boldsymbol{A} & =-\left.\nabla \nabla \log p(\boldsymbol{q} \mid \mathcal{T}, \mathcal{C})\right|_{\boldsymbol{q}=\hat{\boldsymbol{q}}} .
\end{aligned}
$$

In this thesis, the mean function $\mu(\cdot)$ is assumed to be constant, corresponding to a stationary GP, and set to zero. The covariance function is the squared exponential (SE) kernel, $\mathcal{K}_{S E}$, which for two arbitrary points, $\delta$ and $\delta^{\prime}$, is given by

$$
\mathcal{K}_{\mathrm{SE}}\left(\delta, \delta^{\prime}\right)=\sigma_{q}^{2} \exp \left\{-\frac{D_{\delta}^{2}}{2 \Gamma^{2}}\right\},
$$

where $D_{\delta}=\left\|\delta-\delta^{\prime}\right\|$ is a function of input training points and $\Gamma$ and $\sigma_{q}$ are the hyper parameters. While $\sigma_{q}$ controls the amount of deviation around the posterior mean at each point, $\Gamma$ specifies the smoothness of the estimated function. For example, as illustrated in Figure 3.2, a higher value of $\Gamma$ results in highly correlated function values for close points. 


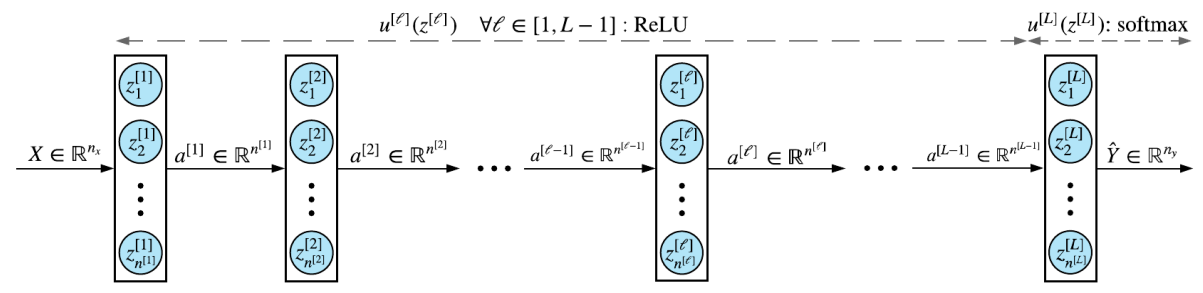

Figure 3.3: The L-layer feed forward neural network structure with $n^{[\ell]}$ number of hidden units in each hidden layer $\ell, \ell \in 1, \ldots, L-1$. The activation functions $u^{[\ell]}\left(z^{[\ell]}\right)$ in all of the hidden layers are $R e L U$ and the activation function in the output layer $u^{[L]}\left(z^{[L]}\right)$ is softmax.

\subsubsection{Feed Forward Neural Network}

The last classifier considered in this thesis is based on feed forward neural network, trained using the $n_{r}$ training examples. A rather shallow, fully connected neural network is developed which outputs probabilities of class memberships given the input vectors computed using the softmax function.

Let $\ell \in[1, \ldots, L]$ denote the number of layers, including hidden layers and the output layer, and $n^{[\ell]}$ denote the number of hidden units, or neurons, in the $\ell:$ th layer of the neural network. The fully connected neural network structure is given in Figure 3.3 in which the output layer, corresponding to the classes defined in Table 7.2, is a vector of size $n_{c}=8$.

For consistency in the derivations that follows, assume that $\ell=0$ corresponds to the input layer, i.e. $\mathbf{X}=a^{[0]}$. Then, the output of each hidden layer, denoted by $a^{[\ell]}$, can be computed by a linear transformation of the previous layer's output $a^{[\ell-1]}$ followed by a nonlinear transformation using an activation function denoted by $u^{[\ell]}(\cdot)$

$$
\begin{aligned}
& z^{[\ell]}=w^{[\ell]} a^{[\ell-1]}+b^{[\ell]}, \\
& a^{[\ell]}=u^{[\ell]}\left(z^{[\ell]}\right),
\end{aligned}
$$

where $w^{[\ell]} \in \mathbb{R}^{n^{[\ell]} \times n^{[\ell-1]}}$ is the weight matrix and $b^{[\ell]} \in \mathbb{R}^{n^{[\ell]} \times 1}$ is the bias vector of the $\ell$ :th layer. To better explain the procedure, Figure 3.4 illustrates the linear and non-linear transformations of a single unit of the $\ell$ :th layer in which $w_{n^{[\ell]}}^{[\ell]}$ denotes the weight vector and $b_{n^{[\ell]}}^{[\ell]}$ denotes the bias scalar corresponding to the $n^{[\ell]}$ :th hidden unit. In this thesis, we use the rectified linear unit (ReLU) as the activation functions for all the hidden layers. For any value $\phi \in \mathbb{R}$, the ReLU activation function is given by

$$
u_{\text {relu }}(\phi)=\max (0, \phi) .
$$

Upon taking the path through all hidden layers $\ell \in[1, \ldots L-1]$, the output of the NN classifier is computed by first passing $a^{[L-1]}$ through the same linear 


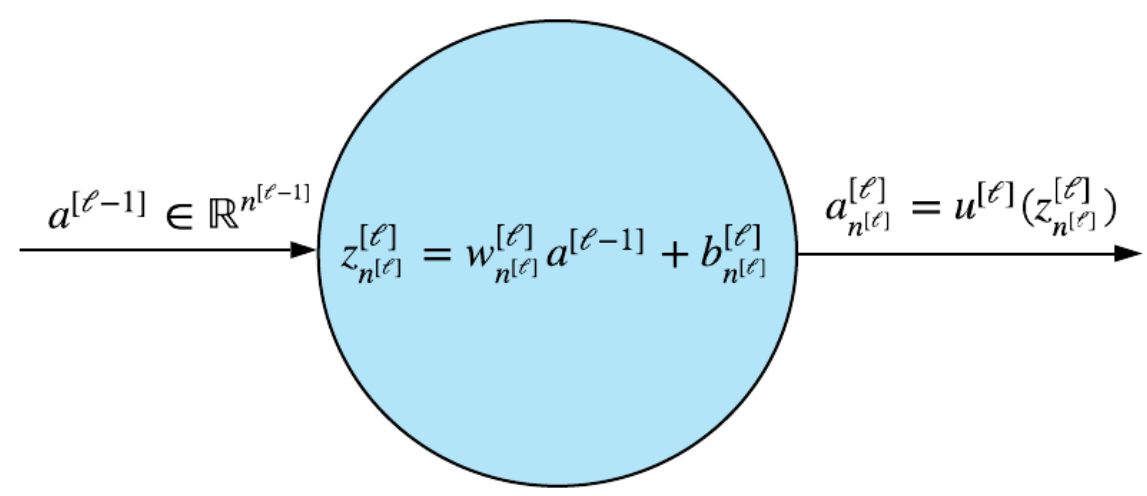

Figure 3.4: Input-output relationship in the $n^{[\ell]}:$ th hidden unit of layer $\ell$.

function (3.12a) to find $z^{[L]}$. A softmax regression layer is then used to estimate the class membership probabilities $a^{[L]}=\hat{\mathcal{C}}=\sigma\left(z^{[L]}\right)$. The unknown weight and bias parameters are learned using back propagation.

After one full forward propagation, in the backward path, an optimization algorithm is employed to find the optimal value of the unknown parameters $w^{[\ell]}$ and $b^{[\ell]}, \ell \in[1, \ldots, L]$ for a given loss function. A softmax classifier is typically trained using the cross-entropy loss function. Given the true classes vector $\mathcal{C}_{r} \in$ $\mathbb{R}^{8}$, one-hot encoded vector, with 1 at the true class and zeros elsewhere, and the estimated class vector $\hat{\mathcal{C}}_{r}$, the loss function, for each training example is given by

$$
\mathcal{L}\left(\hat{\mathcal{C}}_{r}, \mathcal{C}_{r}\right)=-\sum_{c=1}^{8} \mathcal{C}_{c} \log \left(\hat{\mathcal{C}}_{c}\right)+\frac{\lambda}{2 m} \sum_{l=1}^{L}\left\|w^{[l]}\right\|_{F}^{2},
$$

where $\|\cdot\|_{F}$ operator denotes the Frobenius norm of the matrix. The second term on the right hand side of $(3.14 \mathrm{a})$ is the regularization term added to deal with the overfitting problem. Let $\mathcal{L}\left(\hat{\mathcal{C}}_{\mathrm{r}}, \mathcal{C}_{r}\right)$ denote the loss function for all the unknown parameters $\theta=\left\{w_{n^{\ell}}^{[\ell]}, b_{n^{[\ell]}}^{[\ell]}\right\}_{\ell=1}^{L}$. The cost function, given all of the $m$ training examples, can then be obtained by

$$
J(\theta)=\frac{1}{m} \sum_{\tau=1}^{m} \mathcal{L}\left(\hat{\mathcal{C}}_{r}, \mathcal{C}_{r}\right)
$$

In this work, we use the adaptive learning rate optimization (Adam) first introduced in [53] to optimize the cost (3.14b). The Adam optimization algorithm combines the benefits of two optimization methods; gradient descent with momentum [71] and root mean squared prop, RMSprop that is an unpublished adaptive learning rate method proposed by Geoff Hinton in his online course ${ }^{1}$. The

1 http://www.cs.toronto.edu/ tijmen/csc321/slides/lecture_slides_lec6.pdf 
Adam method, in addition to storing an exponentially decaying average of past squared gradients as in RMSprop, also keeps an exponentially decaying average of past gradients, similar to momentum.

Let $d a^{[\ell]}=\frac{\partial J}{\partial a^{\ell \ell]}}$ denote the derivative of the cost function with respect to the activation function at the $\ell$ :th layer. Hence, the derivative of the cost function with respect to the activation function of the last layer initiates the back propagation. For softmax classification, we can easily derive

$$
d a^{[L]}=a^{[L]}-\mathcal{C}^{\operatorname{tr}} .
$$

Computing $d a^{[L]}$ and using the chain rule of derivatives, for each layer $\ell$, one can define

$$
\begin{aligned}
& d z^{[\ell]}=d a^{[\ell]} \frac{\partial a^{[\ell]}}{\partial z^{[\ell]}} \\
& d w^{[\ell]}=d z^{[\ell]} \frac{\partial z^{[\ell]}}{\partial w^{[\ell]}} \\
& d b^{[\ell]}=d z^{[\ell]} \frac{\partial z^{[\ell]}}{\partial b^{[\ell]}} .
\end{aligned}
$$

In order to use the Adam algorithm for finding the optimal values of the unknown parameters, in the backward propagation, the momentum terms $V d w^{[\ell]}$ and $V d b^{[\ell]}$ should be introduced. Furthermore, two additional variables $S d w^{[\ell]}$ and $S d b^{[\ell]}$ corresponding to the RMSprop algorithm are also considered.

At each iteration, using the current mini-batch, the derivative of unknown parameters $d w^{[\ell]}$ and $d b^{[\ell]}$ are first computed. Then, the momentum and RMSprop variables are updated using exponentially weighted average procedure as described in [53]. Finally, the unknown parameters are updated using the updated values of the momentum and RMSprop variables

$$
\begin{gathered}
w^{[\ell]}=w^{[\ell]}-\alpha \frac{V d w^{[\ell]}}{\sqrt{S d w^{[\ell]}}+\epsilon}, \\
b^{[\ell]}=b^{[\ell]}-\alpha \frac{V d b^{[\ell]}}{\sqrt{S d b^{[\ell]}}+\epsilon},
\end{gathered}
$$

where $\alpha$ is the learning rate of the gradient descent algorithm and $\epsilon$ is another hyperparameter of the algorithm mainly for numerical stability. Although the learning rate $\alpha$ needs to be tuned while training the network, the other hyperparameters can be chosen according to the values suggested by the authors in [53], as discussed in Section 7.2.

In addition to the regularization procedure, the proposed method also employs dropout [88] to avoid overfitting the data. For this purpose, during the training phase, $5 \%$ of the hidden units are ignored. The hidden units to be omitted are randomly selected at each epoch. 


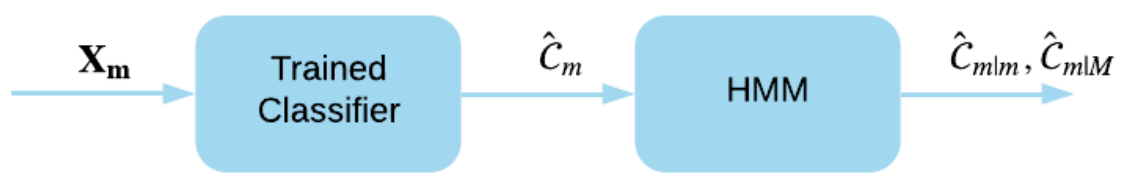

Figure 3.5: The output of the trained neural network, $\hat{\mathcal{C}}_{m}$, is smoothed using an additional HMM block providing a final estimate of the classes, $\hat{\mathcal{C}}_{m \mid M}$.

\subsection{Temporally Correlated Classes}

For the classification problem at hand, we note that there is a high probability that the activity profile and the device mode will remain constant between two consecutive gait cycles. This implies that there is a high temporal correlation between the classes. This additional information can improve the classification rate noticeably and can be modeled using a discrete time, discrete state hidden Markov model as introduced in Section 2.4.

Let $\hat{\mathcal{C}}_{e}, e \in\left\{0, \ldots, n_{e}\right\}$, contain the estimated class membership of the test data samples estimated by any of the classifiers introduced in Sec. 3.2 and $n_{e}=\frac{M-n_{r}}{2}$. It is worth noting that HMM is only applied to the test set. For the sake of simplicity in the notation, we remove the subscript $e$; hence, in what follows, by the $m$ :th gait cycle, we mean the $m$ :th gait cycle in the test set data.

In order to capture the temporal correlation between the classes, as shown in Fig. 3.5, the estimated class membership vectors $\hat{\mathcal{C}}_{m}$ for gait cycle $m$ are fed into an HMM block whose output is the final class membership estimates $\hat{\mathcal{C}}_{m \mid m}$ for filtering and $\hat{\mathcal{C}}_{m \mid M}$ for smoothing approaches.

We let the classification vector $\hat{\mathcal{C}}_{m \mid M}$ denote the hidden states of the HMM and define the observation vector at each time instance based on the classifier output $\hat{\mathcal{C}}_{m}$. The joint distribution of the observation and hidden states can be computed from (2.23).

The emission model is given by the confusion matrix presented in Table 7.3. Additionally, we assume that the states are highly likely to remain in the same regime between the consecutive gait cycles $m-1$ and $m$. This can be captured in the transition matrix $\Pi$ as

$$
\Pi_{i j}= \begin{cases}0.99 & \text { if } i=j \\ \frac{0.01}{n_{c}-1} & \text { otherwise }\end{cases}
$$

where $n_{c}$ is the number of states, here classes, of the HMM model. The classifiers introduced in this section are evaluated using the experimental data in Chapter 7 in which $n_{r}$ training examples together with the development set are used to train multiple classifiers. 


\subsection{Summary}

Basic concepts of machine learning problems, particularly the classification problem, have been introduced in this chapter. Three different classifiers, from a simple $K$-nearest neighbor approach to a more complex neural network solution, were presented and it was shown how the temporal correlation between the classes can be captured. In chapter 7 , the results obtained from applying the trained classifiers introduced in Section 3.2, using a set of novel features, are presented and compared with both filtering and smoothing estimates introduced in Section 3.3. 


\section{Part II}

\section{Applications}





\section{4}

\section{Gait Parameter Estimation}

An earlier version of this application is presented in:

P. Kasebzadeh, C. Fritsche, G. Hendeby, F. Gunnarsson, F. Gustafsson. Improved Pedestrian Dead Reckoning Positioning With Gait Parameter Learning. In Proceedings of the 19th International Conference on Information Fusion, pages 379-385, Heidelberg, Germany, July 2016.

This chapter extends the general PDR algorithm introduced in Chapter 2 to solve a personal navigation problem. The results obtained from the approaches proposed by the author in [48] are presented. Additionally, an extended version of these approaches is investigated in this chapter.

We propose an improved PDR algorithm that learns gait parameters in time intervals when external positioning information is available, for instance from GPS or an indoor positioning system (IPS). Then, the learned gait parameters are used for dead reckoning when no position measurement is available.

Internal gait parameters in the PDR algorithm, such as the step length and the step detection threshold are estimated by a novel filtering approach. The proposed approach is based on a multi-rate Kalman filter bank that estimates the gait parameters when position measurements are available to enhance the performance of the PDR in time intervals when such measurements do not exist. One motivating example is the scenario in which navigation is requested when the user's path contains both outdoor and indoor intervals and the GPS measurements are not always available. The effectiveness of the new approach is investigated on several simulated data as well as data from real world experiments.

We start by introducing PDR models which are extended versions of the model presented in [48] in Section 4.1. In the proposed methods, the step length is considered as a state therefore we need to detect the steps in advance. Section 4.2 introduces the step detection algorithm and step length estimation methods. The 
filtering solution of the considered application is explained in Section 4.3. Section 4.4 discusses the dead reckoning improvements by learning the gait parameters on a number of real-world experiments.

\subsection{Pedestrian Odometry}

In pedestrian navigation systems, the double-integrating strap-down method suffers from an error in the position estimate. This error propagates and increases quadratically with time [98]. Integrating gait parameters could be a solution to this issue. The gait parameters can be utilized in PDR algorithms to improve the performance.

Traditionally, PDR algorithms detect gait parameters, such as the number of detected steps and the step length, in order to determine the travelled distance. This approach is applied in navigation applications in many studies, e.g. $[78,79]$. The basic longitudinal model is given by

$$
d_{s+1}=d_{s}+L+n_{s}
$$

where $d_{s}$ is the total traveled distance, $s$ is the step index, $L$ is the step length and $n_{s}$ is noise. This is a simplified version of the standard model that was introduced in (2.3), considering only one state $d$ with $L$ as an input to the model.

Estimating a pedestrian's step length and determining a suitable threshold for step detection from IMU accelerometer measurements need to be treated appropriately as is discussed in Section 4.2. However, we first introduce the extended versions of the pedestrian odometric model in the following subsections.

\subsubsection{Extended Pedestrian Odometric Model}

States of the extended pedestrian odometric model contain extra components defined by the dimension of the system. One-dimensional models, known as longitudinal models, add only the travelled distance $d$ to the conventional pedestrian odometric model, while in two dimensional horizontal models the step length, angular rate, and bias are also considered.

\section{Longitudinal Model}

A longitudinal multi-rate model is proposed that is updated each time a step is detected or a GPS position estimate becomes available. The underlying motion model is the standard constant velocity (CV) model extended with step length. This model has a state vector $\mathbf{x}=[d, v, L]^{T}$ with travelled distance $d$, velocity $v$ and step length $L$. This model was introduced in [48].

The dynamic model from one event $t_{k}$ to the next event at $t_{k+1}$ is given by either an observed GPS position or a detected step

$$
\mathbf{x}_{t_{k+1}}=\mathbf{F}\left(t_{k+1}-t_{k}\right) \mathbf{x}_{t_{k}}+\mathbf{G}\left(t_{k+1}-t_{k}\right) \mathbf{w}_{t_{k+1}},
$$


where $\mathbf{w}_{t_{k+1}}$ denotes the process noise

$$
\mathbf{w}_{t_{k+1}} \sim \mathcal{N}(0, \mathbf{Q}), \quad \mathbf{Q}=\left[\begin{array}{cc}
\sigma_{a}^{2} & 0 \\
0 & \sigma_{L}^{2}
\end{array}\right] .
$$

The matrices $\mathbf{F}$ and $\mathbf{G}$ are given by

$$
\mathbf{F}(T)=\left[\begin{array}{lll}
1 & T & 0 \\
0 & 1 & 0 \\
0 & 0 & 1
\end{array}\right], \quad \mathbf{G}(T)=\left[\begin{array}{cc}
\frac{T^{2}}{2} & 0 \\
T & 0 \\
0 & 1
\end{array}\right],
$$

where $T$ is the sampling time. In this model, the measurement data is asynchronous, i.e. the time elapsed between two filter updates will depend on which measurement is currently being processed; either a step is detected or a GPS position is available. The general form of the measurement model is given by

$$
y_{k}=\mathbf{H}_{k} \mathbf{x}_{k}+e_{k},
$$

where $\mathbf{H}_{k}$ is a mapping matrix and $e_{k}$ denotes measurement noise. In case a GPS position estimate is available, it is converted to the corresponding velocity as

$$
v_{t_{l}}=\frac{\left\|\hat{\mathbf{p}}_{t_{l}}-\hat{\mathbf{p}}_{t_{l-1}}\right\|}{t_{l}-t_{l-1}}
$$

where $\|\cdot\|$ denotes the $L_{2}$ norm and $\hat{\mathbf{p}}_{t_{l}}$ and $\hat{\mathbf{p}}_{t_{l-1}}$ denote the GPS position estimates available from the current time instance $t_{l}$ and the previous time instance $t_{l-1}$. It is then possible to express the model for the GPS measurement as follows

$$
y_{t_{l}}^{\mathrm{GPS}}=\mathbf{H}_{t_{l}} \mathbf{x}_{t_{l}}+e_{t_{l}}^{\mathrm{GPS}} \text {, }
$$

where $e_{t_{l}}^{\text {GPS }}$ is the noise assumed to be zero-mean Gaussian distributed with variance $\sigma_{\mathrm{v}, \mathrm{GPS}}^{2}$, and $\mathbf{H}_{t_{l}}=[0,1,0]$.

Since there are no direct observations available for step detection, we define an artificial measurement that is used whenever a step is detected (the step detection algorithm is given in Section 4.2). The step detection measurement model is given by

$$
y_{t_{s}}^{\text {step }}=\mathbf{H}_{t_{s}} \mathbf{x}_{t_{s}}+e_{t_{s}}^{\text {step }},
$$

where $\mathbf{H}_{t_{s}}=\left[0, \Delta t_{s},-1\right]$ with $\Delta t_{s}=t_{s}-t_{s-1}$ as the time difference between two consecutive step detections regardless of how many GPS measurements are detected in between, and $e_{t_{s}}^{\text {step }}$ is the noise assumed to be zero-mean Gaussian distributed with variance $\sigma_{\text {step }}^{2}$.

This longitudinal model is linear in the state, and the solution for this problem fits the linear Kalman filter.

\subsubsection{Horizontal Model}

In this section, the two-dimensional multi-rate horizontal models are proposed. The dynamic model is based on a coordinated turn (CT) model with polar velocity extended with step length. A preliminary version of these models has been introduced in [48]. 


\section{Model 1}

The nonlinear CT allows for a varying turn rate and also a varying target speed. The proposed model has a state vector $\mathbf{x}$ with seven components: position $\mathrm{x}$, position $y$, polar velocity $v$, heading $\psi$, heading rate $\omega$, bias $b$, and step length $L$. The resulting differential equation for the state is given by

$$
\dot{\mathbf{x}}=\left[\begin{array}{c}
\dot{\mathrm{x}} \\
\dot{\mathrm{y}} \\
\dot{v} \\
\dot{\psi} \\
\dot{\omega} \\
\dot{b} \\
\dot{L}
\end{array}\right]=\left[\begin{array}{c}
v \cos (\psi) \\
v \sin (\psi) \\
a \\
\omega \\
\alpha \\
0 \\
0
\end{array}\right]
$$

where $a$ is linear acceleration and $\alpha$ is turn rate. The differential equations require discretization in order to apply discrete-time filtering techniques. The result has the form

$$
\mathbf{x}_{t_{k+1}}=\mathbf{f}\left(\mathbf{x}_{t_{k}}, t_{k+1}-t_{k}\right)+\mathbf{g}\left(\mathbf{x}_{t_{k}}, t_{k+1}-t_{k}\right) \mathbf{w}_{t_{k+1}},
$$

where $\mathbf{w}_{t_{k+1}}$ is assumed to be uncorrelated zero-mean Gaussian noise with covariance matrix $\mathbf{Q}=\operatorname{diag}\left(\left[\sigma_{a}^{2}, \sigma_{\alpha}^{2}, \sigma_{L}^{2}\right]\right)$. Discretization of (4.9) yields

$$
\mathbf{f}(\mathbf{x}, T)=\left[\begin{array}{c}
x+\frac{2 v}{\omega} \sin \left(\frac{\omega T}{2}\right) \cos \left(\psi+\frac{\omega T}{2}\right) \\
y+\frac{2 v}{\omega} \sin \left(\frac{\omega T}{2}\right) \sin \left(\psi+\frac{\omega T}{2}\right) \\
v \\
\psi+\omega T \\
\omega \\
b \\
L
\end{array}\right],
$$

where we have assumed $a$ and $\alpha$ to be zero. The zero-order-hold discretization [36] is applied in order to find $\mathbf{g}(\mathbf{x}, T)$ as

$$
\mathbf{g}(\mathbf{x}, T)=\left[\begin{array}{cccc}
\frac{T^{2}}{2} \cos (\psi) & 0 & 0 & 0 \\
\frac{T^{2}}{2} \sin (\psi) & 0 & 0 & 0 \\
T & 0 & 0 & 0 \\
0 & \frac{T^{2}}{2} & 0 & 0 \\
0 & T & 0 & 0 \\
0 & 0 & 1 & 0 \\
0 & 0 & 0 & 1
\end{array}\right]
$$

For the horizontal model it is assumed that the 2D GPS positions, step detection as well as turn rates from gyroscopes are available as measurements. The model for the GPS position estimates is given by

$$
\mathbf{y}_{t_{l}}^{\mathrm{GPS}}=\left[\begin{array}{ccccccc}
1 & 0 & 0 & 0 & 0 & 0 & 0 \\
0 & 1 & 0 & 0 & 0 & 0 & 0
\end{array}\right] \mathbf{x}_{t_{l}}+\mathbf{e}_{t_{l}}^{\mathrm{GPS}},
$$


where $\mathbf{e}_{t_{l}}^{\text {GPS }}$ is noise on the GPS position estimates, which is assumed to be zeromean Gaussian distributed with covariance matrix $\mathbf{R}^{\mathrm{GPS}}=\operatorname{diag}\left(\left[\sigma_{\mathrm{x}, \mathrm{GPS}}^{2}, \sigma_{\mathrm{y}, \mathrm{GPS}}^{2}\right]\right)$. The measurement model for the step detection is analogous to the longitudinal model presented in [48] and is given by

$$
y_{t_{s}}^{\text {step }}=\left[\begin{array}{lllllll}
0 & 0 & \Delta t_{s} & 0 & 0 & 0 & -1
\end{array}\right] \mathbf{x}_{t_{s}}+e_{t_{s}}^{\text {step }}
$$

The gyroscope measurement model is given in (2.2). For convenience, we restate it in a suitable form for the following discussion as

$$
y_{t_{g}}^{\text {gyro }}=\omega_{z, t_{g}}+b_{t_{g}}^{\text {gyro }}+e_{t_{g}}^{\text {gyro }}
$$

where $\omega_{z, t_{g}}$ is the yaw rate which is the gyroscope measurement in the horizontal plane, $b^{\text {gyro }}$ denotes the gyroscope bias, and $t_{g}$ denotes the time instance when gyroscope measurements are available, and $e_{t_{g}}^{\text {gyro }}$ denotes noise assumed to be zero-mean Gaussian with variance $\sigma_{\text {gyro }}^{2}$. It is worth noting that we considered the sensor in body frame, with respect to the navigation frame. The gyroscope measurement model with bias is given by

$$
y_{t_{g}}^{\text {gyro }}=\left[\begin{array}{lllllll}
0 & 0 & 0 & 0 & 1 & 1 & 0
\end{array}\right] \mathbf{x}_{t_{g}}+e_{t_{g}}^{\text {gyro }} .
$$

\section{Model 2}

The proposed model has a state vector $\mathbf{x}$ with eight components: current position $\mathrm{x}^{1}$, current position $\mathrm{y}^{1}$, previous position when step was detected $\mathrm{x}^{2}$, previous position when step was detected $\mathrm{y}^{2}$, polar velocity $v$, heading $\psi$, heading rate $\omega$, and step length $L$. The resulting differential equation for the state is given by

$$
\dot{\mathbf{x}}=\left[\begin{array}{c}
\dot{\mathrm{x}}^{1} \\
\dot{\mathrm{y}}^{1} \\
\dot{\mathrm{x}}^{2} \\
\dot{\mathrm{y}}^{2} \\
\dot{v} \\
\dot{\psi} \\
\dot{\omega} \\
\dot{L}
\end{array}\right]=\left[\begin{array}{c}
v \cos (\psi) \\
v \sin (\psi) \\
0 \\
0 \\
a \\
\omega \\
\alpha \\
0
\end{array}\right],
$$

where $a$ is linear acceleration and $\alpha$ is turn rate. The differential equations require discretization in order to apply discrete-time filtering techniques. The result gets the form

$$
\mathbf{x}_{t_{k+1}}=\mathbf{f}\left(\mathbf{x}_{t_{k}}, t_{k+1}-t_{k}\right)+\mathbf{g}\left(\mathbf{x}_{t_{k}}, t_{k+1}-t_{k}\right) \mathbf{w}_{t_{k+1}},
$$


where $\mathbf{w}_{t_{k+1}}$ is assumed to be uncorrelated zero-mean Gaussian noise with covariance matrix $\mathbf{Q}=\operatorname{diag}\left(\left[\sigma_{a}^{2}, \sigma_{\alpha}^{2}, \sigma_{L}^{2}\right]\right)$. The discretization of (4.17) yields

$$
\mathbf{f}(\mathbf{x}, T)=\left[\begin{array}{c}
\mathrm{x}^{1}+\frac{2 v}{\omega} \sin \left(\frac{\omega T}{2}\right) \cos \left(\psi+\frac{\omega T}{2}\right) \\
\mathrm{y}^{1}+\frac{2 v}{\omega} \sin \left(\frac{\omega T}{2}\right) \sin \left(\psi+\frac{\omega T}{2}\right) \\
\mathrm{x}^{2} \\
\mathrm{y}^{2} \\
v \\
\psi+\omega T \\
\omega \\
L
\end{array}\right],
$$

where we have assumed $a$ and $\alpha$ to be zero. The zero-order-hold discretization [36] is applied in order to find $g(\mathbf{x}, T)$ which is given by

$$
\mathbf{g}(\mathbf{x}, T)=\left[\begin{array}{ccc}
\frac{T^{2}}{2} \cos (\psi) & 0 & 0 \\
\frac{T^{2}}{2} \sin (\psi) & 0 & 0 \\
0 & 0 & 0 \\
0 & 0 & 0 \\
T & 0 & 0 \\
0 & \frac{T^{2}}{2} & 0 \\
0 & T & 0 \\
0 & 0 & 1
\end{array}\right] .
$$

For the horizontal model it is also assumed that 2D GPS position estimates, step detection as well as turn rates from gyroscopes are available as measurements. The model for the GPS position estimates is given by

$$
\mathbf{y}_{t_{l}}^{\mathrm{GPS}}=\left[\begin{array}{cccccccc}
1 & 0 & 0 & 0 & 0 & 0 & 0 & 0 \\
0 & 1 & 0 & 0 & 0 & 0 & 0 & 0
\end{array}\right] \mathbf{x}_{t_{l}}+\mathbf{e}_{t_{l}}^{\mathrm{GPS}},
$$

where $\mathbf{e}_{t_{l}}^{\text {GPS }}$ is noise on the GPS position estimates, which is assumed to be zeromean Gaussian distributed with covariance matrix $\mathbf{R}^{\mathrm{GPS}}=\operatorname{diag}\left(\left[\sigma_{\mathrm{x}, \mathrm{GPS}}^{2}, \sigma_{\mathrm{y}, \mathrm{GPS}}^{2}\right]\right)$.

The gyroscope measurement model is given as

$$
y_{t_{g}}^{\text {gyro }}=\omega_{z, t_{g}}+e_{t_{g}}^{\text {gyro }},
$$

where the only difference with (4.15) is that the bias is neglected in this case because it is compensated via calibration. Hence

$$
y_{t_{g}}^{\text {gyro }}=\left[\begin{array}{llllllll}
0 & 0 & 0 & 0 & 0 & 0 & 1 & 0
\end{array}\right] \mathbf{x}_{t_{g}}+e_{t_{g}}^{\text {gyro }} .
$$

Since there are no direct observations for step detections available, we define an artificial measurement. The measurement model for the step length is given by

$$
y_{t_{s}}^{\text {step }}=\mathbf{H} \mathbf{x}_{t_{s}}+e_{t_{s}}^{\text {step }}
$$


where $\mathbf{H}$ denotes the gradient of $\mathbf{H}=h^{\prime}(x)$ and $h(x)=\left\|p_{2}-p_{1}\right\|-L$. Here, $H$ is given by

$$
\mathbf{H}=\left[\begin{array}{llllllll}
\frac{\partial h(x)}{\partial x^{1}} & \frac{\partial h(x)}{\partial y^{1}} & \frac{\partial h(x)}{\partial x^{2}} & \frac{\partial h(x)}{\partial y^{2}} & 0 & 0 & 0 & \frac{\partial h(x)}{\partial L}
\end{array}\right]
$$

In contrast to the longitudinal model, the horizontal model is nonlinear in the states due to the nonlinear process model. Hence, nonlinear filters have to be used for state estimation, and we will make use of the Extended Kalman filter, presented in Section 2.3.4, in our proposed PDR solution.

\subsection{Step Detection}

Step detection is one of the challenges of using PDR algorithm for navigating pedestrians. Here, we overview the recent research work that have been done concerning this problem and then propose an algorithm for estimating the gait parameter.

The most used signals for step detection purposes are those corresponding to acceleration and angular rate. Depending on the application at hand, two detection algorithms are used. In scenarios with body-mounted devices, static detection is performed while in case of hand-held devices zero-cross detection and peak detection are applied. One source of error in these methods is due to multiple peak values per step. To address this false detection problem $[61,78$, $83,84,92]$, look at the norm of the total acceleration rather than looking only at the vertical acceleration component.

Another possible source of error in step detection algorithms is the orientation of the sensor that is used for measuring signals. To avoid disturbances caused by this arbitrary orientation on the vertical signal component, [46] projects acceleration in local coordinate system to a global one. In this way, it is possible to resolve issues due to the placement of the device.

Accurate estimation of the number of steps from the peaks of the accelerometer signal could be improved by filtering the signal. That is, one can modify the output waveform of the tri-axial accelerometer and then count the number of peaks. In this regard, [61] considers a band-pass filtering approach. Signal segmentation, by means of windowing techniques, is also studied in the literature. [102] applies a filter with a low and high cut-off value. Then, by comparing steps and the threshold value, the number of steps is estimated.

Pre-processing the raw accelerometer signal is investigated in [46]. After the pre-processing phase, a high-pass filter is applied to the acceleration in the $z$ axis in global coordinates to remove the effects of the Earth's gravity. Then, a low-pass filtering procedure is used to remove the high frequency random noise components.

In case of hand-held devices, extra attention is needed as the device might be swinging in the user's hand. The swinging pattern generates incorrect peaks that need to be addressed. [61] introduces two thresholds $\tau$ and $\sigma$ for time and peaks to remove the errors involved in step counting. Depending on the sampling 
frequency, data analyses, and test results, they adjust values of these thresholds. Taking advantages of the motion pattern is another solution proposed in [84]. The authors apply different peak thresholds in different step detection algorithms based on the motion pattern. In [78], it is shown that it is possible to use motion recognition first and then use accelerometer and gyroscope signal in order to detect the steps.

\subsubsection{Step Detection Algorithm}

A large number of studies consider the three-axis accelerometer signal as a sufficient source of information required to detect the occurrence of a step. The vertical axis, relative to the ground, contains all information corresponding to the step-specific peak. However, the orientation of the sensor may cause some disturbances on the vertical signal component. One typical example is when the IMU is embedded in hand-held devices such as smartphones. In order to avoid disturbances, the norm of the total acceleration is used instead of looking only at the vertical acceleration component.

Algorithm 1 Step Detection [48].

- Input: Three-axis Accelerometer Signal

1. Compute the norm of the accelerometer signal:

$$
a=\sqrt{a_{x}^{2}+a_{y}^{2}+a_{z}^{2}} .
$$

2. Band-pass filter the resulting signal.

3. If the filtered signal exceeds a defined threshold $h$, a step is detected.

4. Among all sets of accelerations that are larger than the threshold, before the signal drops below the threshold again, the one with the highest value is selected as the step.

- Output: Step detections

Algorithm 1 illustrates the step detection algorithm. In this algorithm, the variables $a_{x}, a_{y}$, and $a_{z}$ denote the accelerometer components. In order to improve the quality of the signal, a Butterworth band-pass filter with a proper cutoff frequency is applied to attenuate all frequencies outside the desired range.

Defining a suitable threshold $h$ is a non-trivial and important task. The threshold needs to be adjusted for different people with different characteristics. In order to make our point clear, we have designed an experiment in which three people with different attributes (height and weight) and walking behaviors have participated. All of them walked along the same rectangular trajectory. In order to have a better classification per person, they were asked to walk in a slow 


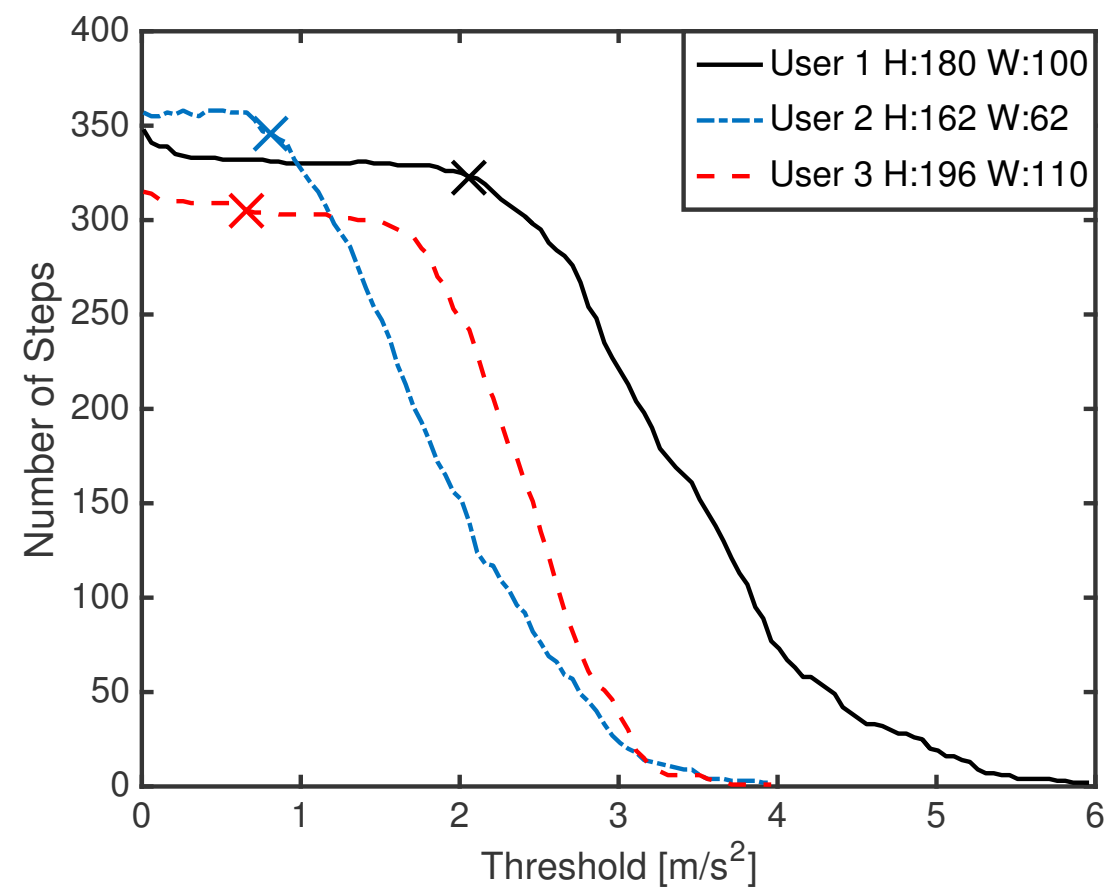

Figure 4.1: Number of steps detected versus the step detection threshold $h$ for users with different height $(H)$ and weight $(W)$. The crosses indicate true number of steps [48, O2016 IEEE].

pace and count their steps, so that these can be used as the ground truth. As Figure 4.1 shows the threshold for each user is unique. Furthermore, choosing a too large threshold will underestimate the number of detected steps. For instance, the number of detected steps for user 2 drops rapidly for larger thresholds.

Different walking speeds might also affect the optimal threshold value. Figure 4.2 illustrates the step detection performance for different paces of a single user. We observe that different speeds yield different step detection curves, hence choosing the right threshold becomes important.

It is worth noting that in all of our experiments the user is walking during the whole data collection experiment. This means that below a certain threshold, the step detection curves become (relatively) flat. Hence, one could argue that a properly chosen (small enough) fixed threshold might be sufficient to obtain good step detection performance. However, in reality the user is not walking all the time. The user motion will rather be interrupted by shorter (or longer) periods of stand still, resulting in step detection curves that are no longer flat and would result in a significant increase of false detections, if the threshold is chosen too small. The effect of different motion modes is illustrated in Figure 4.3 where two users were asked to walk with different modes and as in the previous 


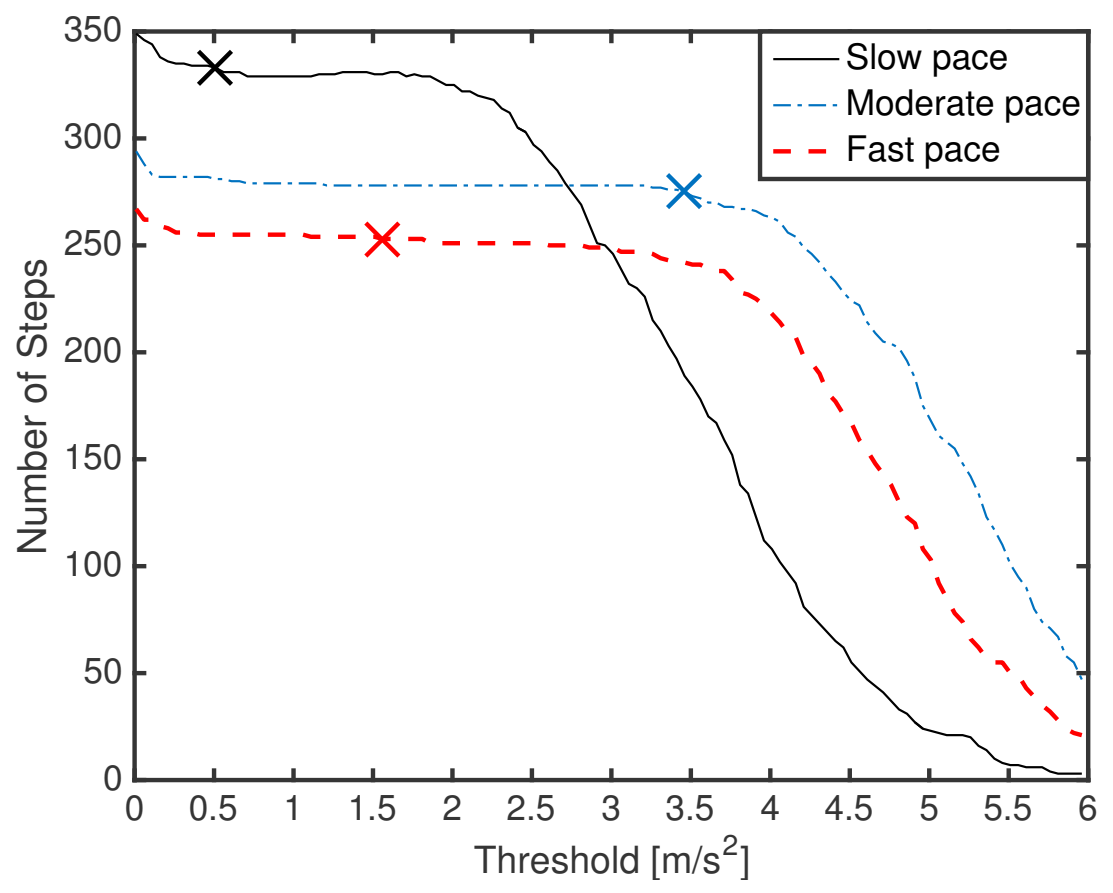

Figure 4.2: Number of steps detected versus the step detection threshold $h$ for one user with different walking modes. The crosses indicate true number of steps [48, C2016 IEEE].

case, the ground truth is their counts of taken steps. The step detection curves are no longer flat and a too small threshold results in too many false detections.

\subsubsection{Step Length Estimation}

Lately, much research has been conducted on step length estimation [41, 46, $61,92]$. One method for estimating the step length is to consider the relations between output waveform of the tri-axial accelerometer and step length, also known as the empirical formula. In this method, the step length is computed by using the difference between maximum and minimum output waveform of a tri-axial accelerometer in each step. Then, a calibration factor based on the ratio of the real and the estimated reference trajectory distances must be applied.

The estimation approach using an empirical formula and back propagation (BP) neural network is studied in [61, 92]. In [41], the calibration factor of the empirical formula is selected adaptively. That is, instead of using a constant factor for all steps, a polynomial function of the average step velocity is used for derivation of the calibration factor. The degree of the polynomial is then given by an $N$-fold cross-validation being applied to the dataset. 


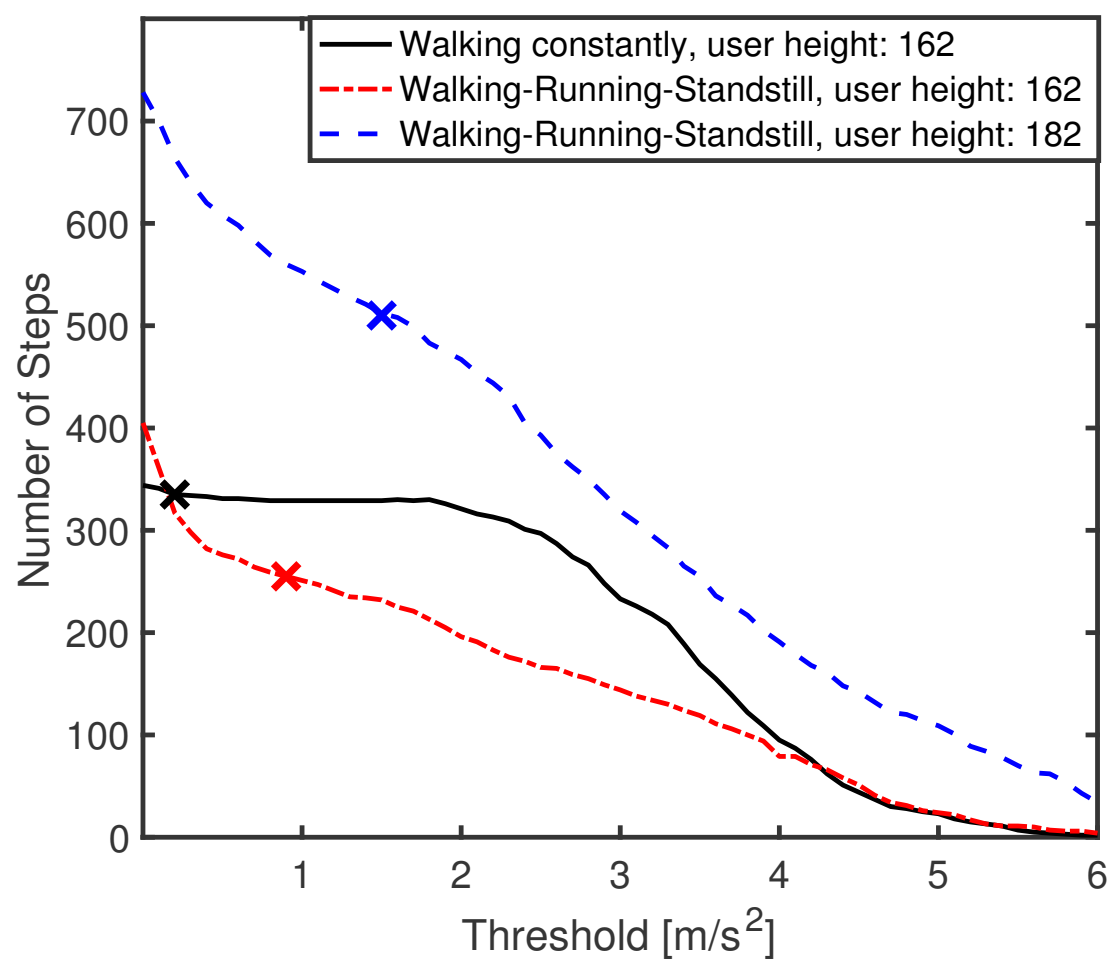

Figure 4.3: Number of steps detected versus the step detection threshold $h$ for a single user with different walking paces. The crosses indicate true number of steps.

Linear formula is another common method for step length estimation. In case of walking or running pedestrians, [102] applies the linear formula to estimate the step length. Pre-initialized parameters required in this method are obtained by analyzing a large amount of experiment data.

Depending on the motion pattern, [102] uses different formulas to estimate the step length in an application that contains going up or down the stairs. The horizontal length of a stair and the threshold determined by the step speed are used for step length estimation.

A method based on short time Fourier transform (STFT) is introduced in [78]. Independent of the step detection process, the authors use STFT to extract the frequency content of the device's signal. Based on the detected frequency and linear relationship between frequency and step length, the step length is estimated.

In our proposed algorithm, the step length is considered as a state in our model and will be estimated both online and offline using multi-rate filtering in a filter bank framework. That is, the step length estimation process does 
not require any pre-initialized parameters and is compatible with the pedestrian speeds which may vary due to changing the walking pattern.

\subsection{Filter Bank}

There are applications where the system needs to have multiple operational models. Given the true model, it is possible to use an appropriate filter at each mode of the operation. However, since the true mode is unknown, a filter bank enumerates all possible combinations and keeps track of their likelihood.

The generic model (2.6) then needs to be extended to include the mode parameter as

$$
\begin{aligned}
\mathbf{x}_{k+1} & =\mathbf{f}_{k}\left(\mathbf{x}_{k}, \mathbf{w}_{k}, \boldsymbol{\delta}_{k}\right), \\
\mathbf{y}_{k} & =\mathbf{h}_{k}\left(\mathbf{x}_{k}, \mathbf{e}_{k}, \boldsymbol{\delta}_{k}\right),
\end{aligned}
$$

where $\delta_{k}$ denotes the mode at time instant $k$. The filter bank treats different filters individually. Assuming that each filter is the correct one, the estimator computes the likelihood of each filter. The state estimates obtained from all the filters are then blended and weighted by their likelihood. One challenge in constructing filter banks is to avoid exponential growth of the number of possible mode combinations using a mode reduction technique.

The mode reduction methods are based on pruning or merging the tree of the mode sequences. Keeping track of the likelihood of each mode combination, pruning reduces the size of a filter bank by discarding unlikely mode combinations. Merging, on the other hand, combines the branches with the same history over a window of a specific length.

The pruning and/or merging can be performed in different ways [36, 38]. Interacting multiple model (IMM), Generalized peseudo-Bayesian filter [16], and the adaptive forgetting through multiple models [7] are among typical available methods.

The filter bank that is used in this work is tailored to our case and is slightly different from the standard form of multiple model adaptive estimators. The number of the parallel filters is fixed and the true threshold is considered as an unknown parameter and parallel filters run with different hypotheses. Subsequently, all mode probabilities need to be evaluated to find the best-fit threshold.

The mode probabilities are estimated by the filter bank in both offline and online fashions. In the offline case, the threshold has been estimated by minimizing a likelihood cost function where the proposed approach processes batches of real data for each experiment. The result of this work have been presented in [48]. In the online case, the proposed approach processes the data sequentially and all mode probabilities are merged to find the best fit threshold at each time. IMM filter is the method that is used for merging the mode probabilities in our study. The transition probability matrix (TPM), $\pi$, in IMM filter is assumed to be known. In the rest of this section, offline and online filter bank estimators are described in more details. 


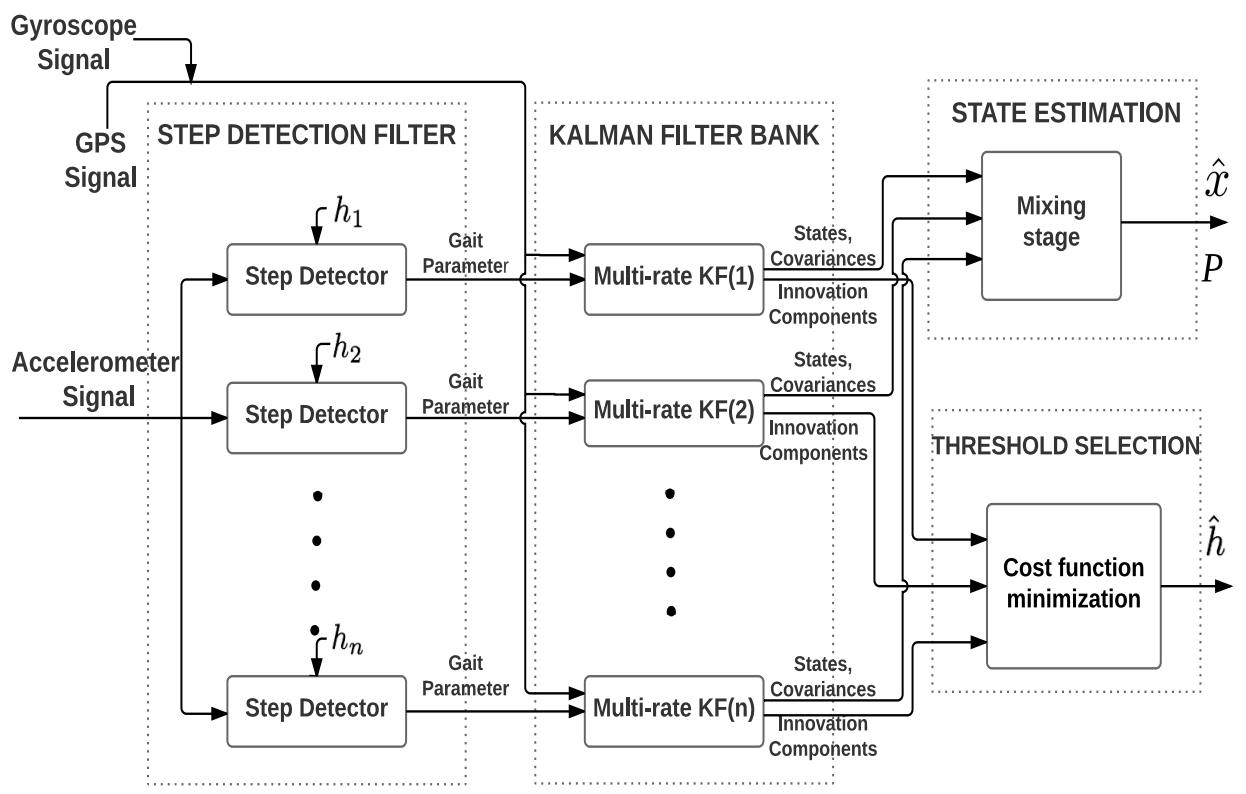

Figure 4.4: Block diagram of PDR algorithm with gait parameter learning [48, C2016 IEEE].

\subsubsection{Offline Kalman Filter Bank}

The block diagram of the proposed PDR algorithm that learns gait parameters is shown in Figure 4.4. The algorithm consists of four blocks. In the step detection filter block, $N$ different thresholds are applied to a bank of step detectors, whose inputs are the accelerometer signals. The detected steps, as the output of this filter, are passed to the Kalman filter bank block consisting of $N$ multi-rate (Extended) Kalman filters, that process the step detections, GPS position estimates (and gyroscope measurements). The results of this filter bank are then passed to the state estimation and threshold selection block.

The threshold selection block is responsible for processing $N$ threshold dependent innovation vectors $\varepsilon_{k}(h)$ and corresponding innovation covariances $\mathbf{S}_{k}(h)$ to obtain a threshold estimate $\hat{h}$ for the step detector based on minimizing some likelihood cost function. In the state estimation block, the $N$ estimated states together with the error covariances and other filter parameters are passed as inputs to a mixing stage that produces the final filter output consisting of a state vector estimate $\hat{\mathbf{x}}$ and the corresponding error covariance $\mathbf{P}[36]$.

While the proposed approach should be fully capable of processing the data sequentially, the majority of blocks could be only tested by processing batches of real data for each experiment. In particular, all blocks except the state estimation block, were each processed offline using batches of data. This means that the step detection filter processed all data at once. The output of this filter, a batch 


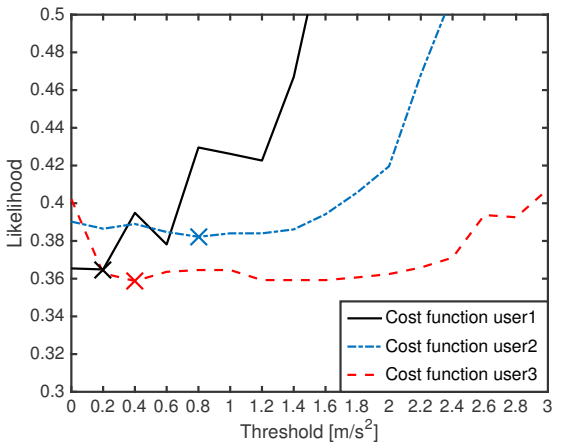

(a) Three different users with different characteristics.

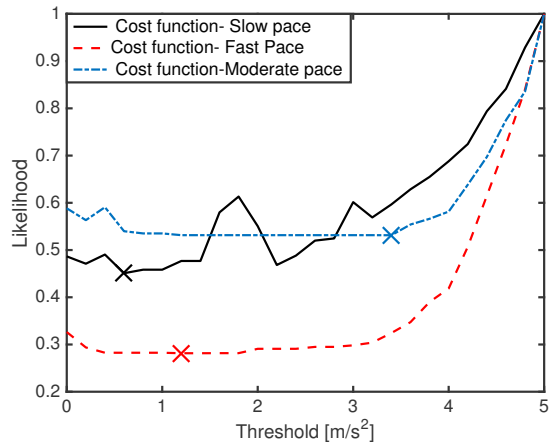

(b) A single user with three different walking paces.

Figure 4.5: Likelihood cost function against the step detection threshold $h$. The crosses indicate the estimated threshold $\hat{h}$ where the likelihood cost function is minimized [48, (C2016 IEEE].

of detected steps, was then fed into the Kalman filter bank to output a batch of innovations and covariances. The threshold estimation block has been adapted to process batches of data. More specifically, the threshold estimate $\hat{h}$ for each experiment is obtained by minimizing the following likelihood cost function

$$
\hat{h}=\arg \min _{h} \frac{\sum_{k=1}^{N} \varepsilon_{k}^{T}(h) \mathbf{S}_{k}^{-1}(h) \varepsilon_{k}(h)+\log \mathbf{S}_{k}(h)}{N},
$$

where $N$ is the length of the batch. A small $h$, gives many false detected steps resulting in large innovations. Conversely, a large $h$ results in missed detection, yielding large innovations again. While the proposed analysis is not fully sequential, it gives a very good insight on the potential of the proposed approach for PDR and gait parameter learning.

Figure $4.5 \mathrm{a}$ and $4.5 \mathrm{~b}$ present results for the threshold selection block. Here, the (batch) of measurement data corresponding to three different users all walking slowly but with different physical characteristics and the data corresponding to the single user with different walking paces have been processed. The data batches used here are identical to the ones that have been used for generating Figure 4.1 and Figure 4.2. It can be observed that all cost functions are different and yield different estimated $\hat{h}$ values when the likelihood cost function is minimized.

In Table 4.1, the estimated thresholds from minimizing the likelihood cost function are compared to the "true" thresholds obtained from counting the total number of steps during the experiments. It can be observed that the estimated thresholds $\hat{h}$ are in good agreement with the true ones, and hence the criterion given in (4.27) seems to be a good indicator to choose the step detection threshold. 
Table 4.1: Estimated step detection threshold $\hat{h}$ versus true threshold [48].

\begin{tabular}{|c|c|c|}
\hline & $\begin{array}{c}\text { True Threshold } \\
{\left[\mathrm{m} / \mathrm{s}^{2}\right]}\end{array}$ & $\begin{array}{c}\text { Estimated threshold } \\
{\left[\mathrm{m} / \mathrm{s}^{2}\right]}\end{array}$ \\
\hline User 1 & 2.06 & 2.0 \\
\hline User 2 & 0.81 & 0.8 \\
\hline User 3 & 0.61 & 0.4 \\
\hline Slow pace & 0.51 & 0.6 \\
\hline Moderate pace & 3.46 & 3.4 \\
\hline Fast pace & 1.56 & 1.4 \\
\hline
\end{tabular}

\subsubsection{Online Kalman Filter Bank}

This section proposes an online PDR algorithm featuring gait parameters learning using IMM. In order to simplify the procedure first a single step of the IMM filter is presented as an algorithm and then the whole algorithm is presented in a block diagram and each block is described in detail.

\section{IMM Filter}

Let $\left\{\hat{\mathbf{x}}_{k \mid k}^{j}, \mathbf{P}_{k \mid k}^{j}, \mu_{k}^{j}\right\}_{j=1}^{N}$ denote the previous sufficient statistics. Then, a single step of the IMM algorithm to obtain current sufficient statistics $\left\{\hat{\mathbf{x}}_{k+1 \mid k+1}^{i}, \mathbf{P}_{k+1 \mid k+1}^{i}, \mu_{k+1}^{i}\right\}_{i=1}^{N}$ is described in the sequel in Algorithm $2[9,10]$.

The block diagram of the proposed online PDR algorithm featuring gait parameters learning is shown in Figure 4.6. The block diagram consists of six blocks. The "mixing probability calculation" block processes $N$ mode probabilities $\mu_{k}^{i}$ to obtain mixing probability $\mu_{k}^{j i}$ presented in the "Mixing" step in Algorithm 2. The "Mixing block" mixes the state estimates and their covariances using the estimated probabilities. The computed mixed statistics together with the detected steps, extracted by the "step detection" block, are then passed to the Kalman filter bank.

The output calculation step in Algorithm 2 gives the output of the whole process. However, it does not affect the sufficient statistics for the next loop as illustrated in "output estimation calculation" block in Figure 4.6.

\subsection{Dead Reckoning in Different Environments}

All the proposed approaches in this chapter are evaluated by both simulated and real field data. 


\section{Algorithm 2 IMM Filter $[9,95]$}

- Input: previous sufficient statistics

- Mixing: Compute the mixing probability $\mu_{k}^{j i}$, mixed estimates $\hat{\mathbf{x}}_{k \mid k}^{0 i}$ and covariances $\mathbf{P}_{k \mid k}^{0 i}$ :

$$
\begin{aligned}
\mu_{k \mid k}^{j i} & =\frac{\pi_{j i} \mu_{k}^{j}}{\sum_{l=1}^{N} \pi_{l i} \mu_{k}^{l}}, \\
\hat{\mathbf{x}}_{k \mid k}^{0 i} & =\sum_{j=1}^{N} \mu_{k \mid k}^{j i} \hat{\mathbf{x}}_{k \mid k}^{j}, \\
\mathbf{P}_{k \mid k}^{0 i} & =\sum_{j=1}^{N} \mu_{k \mid k}^{j i}\left[\mathbf{P}_{k \mid k}^{j}+\left(\hat{\mathbf{x}}_{k \mid k}^{j}-\hat{\mathbf{x}}_{k \mid k}^{0 i}\right)\left(\hat{\mathbf{x}}_{k \mid k}^{j}-\hat{\mathbf{x}}_{k \mid k}^{0 i}\right)^{T}\right] .
\end{aligned}
$$

- Mode Matched Prediction Update: Compute the $\hat{\mathbf{x}}_{k+1 \mid k}^{i}$ and $\mathbf{P}_{k+1 \mid k}^{i}$

$$
\begin{aligned}
\hat{\mathbf{x}}_{k+1 \mid k}^{i} & =\mathbf{F}(i) \hat{\mathbf{x}}_{k \mid k}^{0 i}, \\
\mathbf{P}_{k+1 \mid k}^{i} & =\mathbf{F}(i) \mathbf{P}_{k \mid k}^{0 i} \mathbf{F}^{T}(i)+\mathbf{G}(i) \mathbf{Q G}{ }^{T}(i) .
\end{aligned}
$$

- Mode Matched Measurement Update: Compute the update estimate $\hat{\mathbf{x}}_{k+1 \mid k+1}$, covariance $\mathbf{P}_{k+1 \mid k+1}$ and update the mode probability $\mu_{k+1}^{i}$

$$
\begin{aligned}
\hat{\mathbf{y}}_{k+1 \mid k}^{i} & =\mathbf{H}(i) \hat{\mathbf{x}}_{k+1 \mid k}^{i}, \\
\mathbf{S}_{k+1}^{i} & =\mathbf{H}(i) \mathbf{P}_{k+1 \mid k}^{i} \mathbf{H}(i)^{T}+\mathbf{R}, \\
\mathbf{K}_{k+1}^{i} & =\mathbf{P}_{k+1 \mid k}^{i} \mathbf{H}^{T}(i)\left(\mathbf{S}_{k+1}^{i}\right)^{-1}, \\
\hat{\mathbf{x}}_{k+1 \mid k+1}^{i} & =\hat{\mathbf{x}}_{k+1 \mid k}^{i}+\mathbf{K}_{k+1}^{i}\left(\mathbf{y}_{k}-\hat{\mathbf{y}}_{k+1 \mid k}^{i}\right), \\
\mathbf{P}_{k+1 \mid k+1}^{i} & =\mathbf{P}_{k+1 \mid k+1}^{i}-\mathbf{K}_{k+1}^{i} \mathbf{S}_{k+1}^{i} \mathbf{K}_{k+1}^{i T}, \\
\mu_{k+1}^{i} & =\frac{\mathcal{N}\left(\mathbf{y}_{k+1} ; \hat{\mathbf{y}}_{k+1 \mid k}^{i}, \mathbf{S}_{k+1}^{i}\right) \sum_{j=1}^{N} \pi_{j i} \mu_{k}^{j}}{\sum_{l=1}^{N} \mathcal{N}\left(\mathbf{y}_{k+1} ; \hat{\mathbf{y}}_{k+1 \mid k}^{l}, \mathbf{S}_{k+1}^{l}\right) \sum_{j=1}^{N} \pi_{j l} \mu_{k}^{j}} .
\end{aligned}
$$

- Output Estimate Calculation: Compute the overall $\hat{\mathbf{x}}_{k+1 \mid k+1}$ and $\mathbf{P}_{k+1 \mid k+1}$

$$
\begin{aligned}
\hat{\mathbf{x}}_{k+1 \mid k+1} & =\sum_{i=1}^{N} \mu_{k+1}^{i} \hat{\mathbf{x}}_{k+1 \mid k+1}^{i}, \\
\mathbf{P}_{k+1 \mid k+1} & =\sum_{i=1}^{N} \mu_{k+1}^{i}\left[\mathbf{P}_{k+1 \mid k+1}^{i}+\left(\hat{\mathbf{x}}_{k+1 \mid k+1}^{i}-\hat{\mathbf{x}}_{k+1 \mid k+1}\right)\left(\hat{\mathbf{x}}_{k+1 \mid k+1}^{i}-\hat{\mathbf{x}}_{k+1 \mid k+1}\right)^{T}\right] .
\end{aligned}
$$

- Output: current sufficient statistics. 


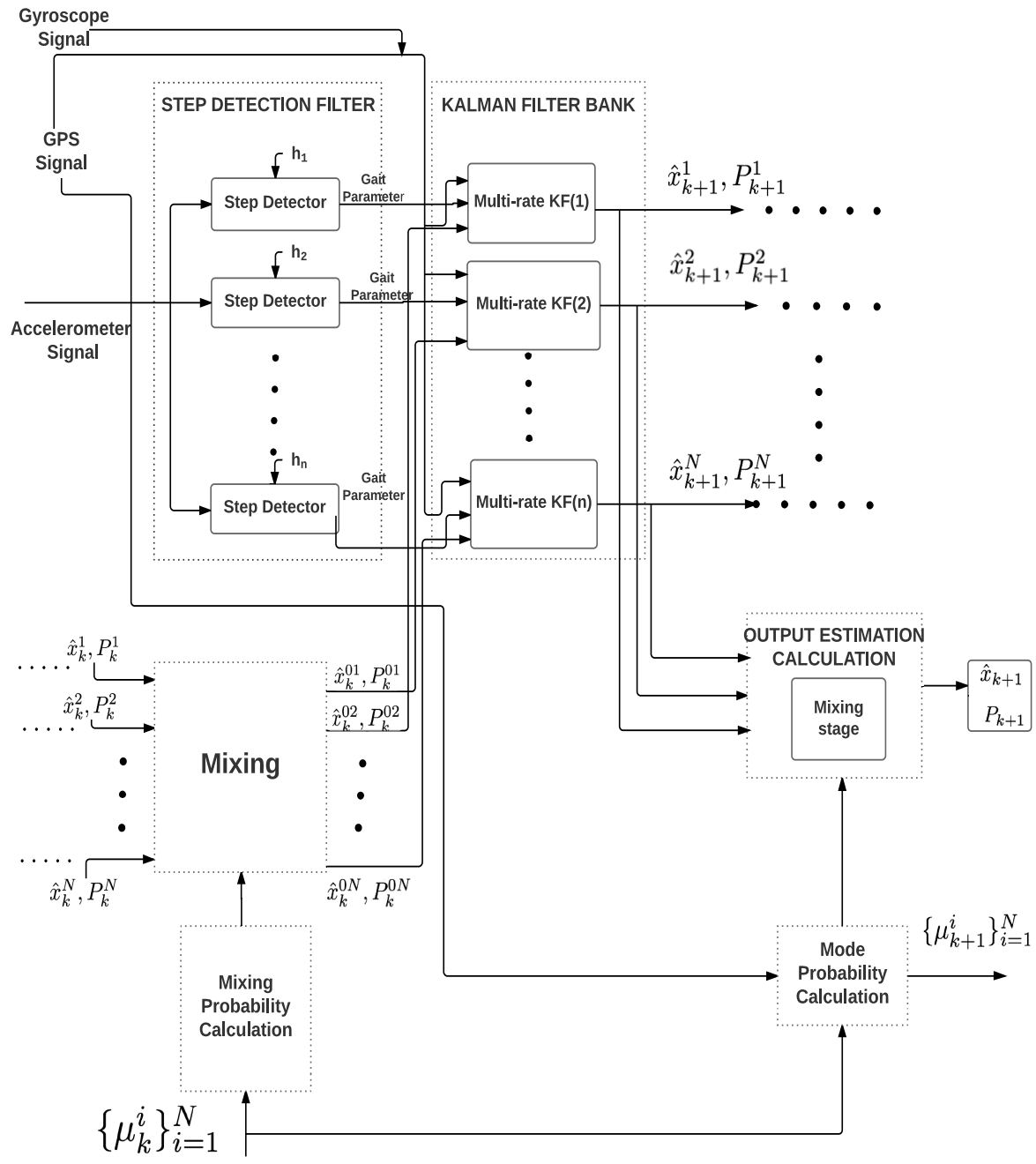

Figure 4.6: Block diagram of one step of the online Kalman filter bank using IMM algorithm for $\mathrm{N}$ models. 


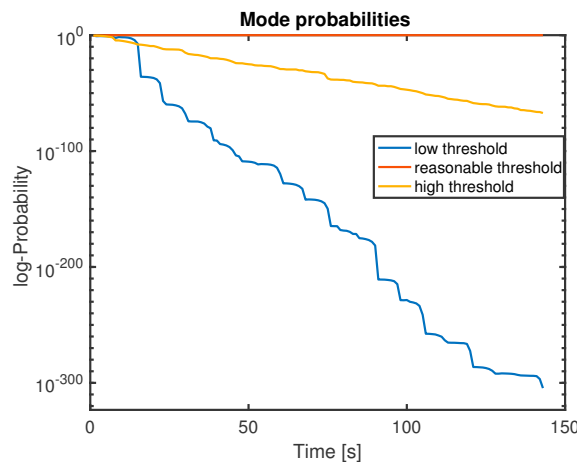

(a) Probability mode for three different thresholds

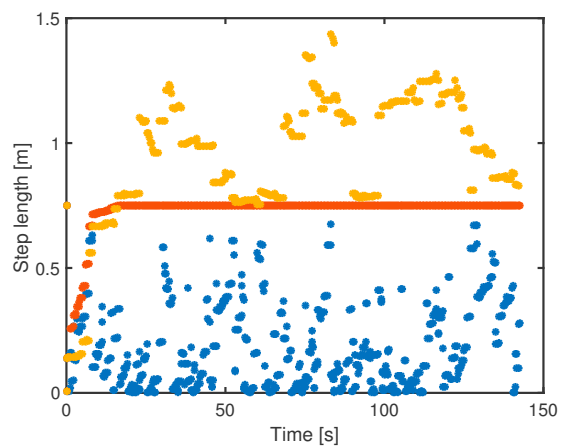

(b) Estimated step length for three different thresholds.

Figure 4.7: The results are the "Kalman Filter bank" block outputs represented in Figure 4.6. The data is generated for the longitudinal model and the online Kalman filter bank approach is used.

\subsubsection{Simulated Environment}

Generated data is used to verify the models under perfect conditions without noise. It also allows for using the true value for the parameter as reference and compare with the estimate.

\section{Longitudinal Model}

The results of the simulated data for the longitudinal model are represented in Figure 4.7 and Figure 4.8. Figure 4.7 shows the mode probabilities and the estimated step lengths for three different thresholds. The true step length in this scenario is $0.75 \mathrm{~m}$. The process noise covariance matrix for this model is $\mathbf{Q}=\operatorname{diag}([0.1,0.001])$ and the measurement noise for GPS and step length are $0.1 \mathrm{~m}$ and $0.01 \mathrm{~m}$, respectively.

Setting a reasonable threshold results in accurate step length estimates. Meanwhile, applying a very low/high threshold makes the estimated quantities to diverge from true value. Figure 4.7a indicates the "reasonable threshold" with solid red line which has the highest probability and, as shown in Figure 4.7b, the step length estimation is highly accurate.

By mixing the threshold results, the step lengths and the traveled distances are estimated accurately as illustrated in Figure 4.8. Fig 4.8a represents the step length estimation results which is the output of the "output estimation calculation" block in Figure 4.6. Moreover, Figure $4.8 \mathrm{~b}$ presents the root mean square error of the estimated travelled distance. 


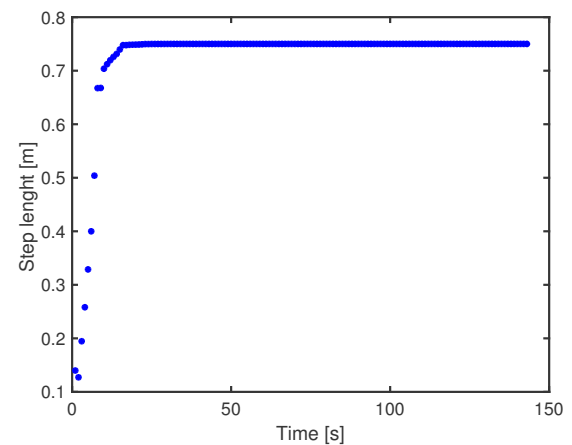

(a) Estimated step length by mixing hypotheses for the thresholds. The results is from the "output estimation calculation" block in Figure 4.6.

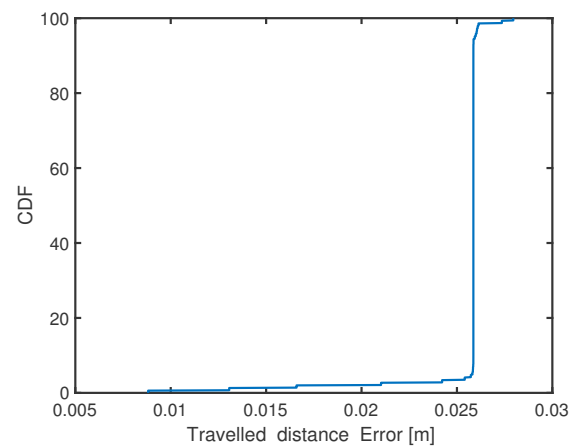

(b) The CDF of root mean square error for estimated travelled distance.

Figure 4.8: The data is generated for the longitudinal model and the Kalman filter bank approach is used.

\section{Horizontal Model}

Data for the the horizontal model is also generated and used for performance evaluations. The true step length in this scenario is also assumed to be $0.75 \mathrm{~m}$. The process noise covariance matrix for this model is $\mathbf{Q}=\operatorname{diag}([0.1,0.1,0.001])$ and the measurement noise standard deviation for GPS, gyroscope and step length are $0.1,0.2$, and 0.01 , respectively. The thresholds are set with the same value as in the longitudinal model. In Figure 4.9a the "reasonable threshold" indicated with solid red line represents the highest probability. The estimated step lengths, presented in Figure 4.9b, for this threshold are the closest to the true step length.

By mixing the threshold results, the step lengths and position are estimated accurately as illustrated in Figure 4.10. Figure 4.10a presents the step length estimation results which is the output of the "output estimation calculation" block in Figure 4.6. Moreover, Figure 4.10b presents the estimated position over the true position. The results indicate that the estimated trajectory follows the true path.

\subsubsection{Real Environment}

In this section, the performance of the models introduced in Section 4.1.1 are evaluated using real field experiments. The result obtained from the 2-D models are also compared and advantages and disadvantages of each model are discussed. 


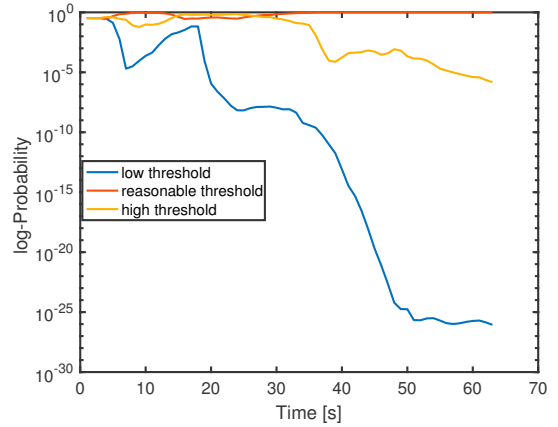

(a) Probability mode for three different thresholds.

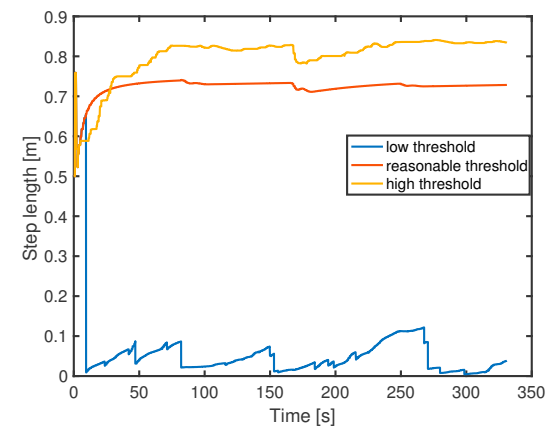

(b) Estimated step length for three different thresholds.

Figure 4.9: The results of the "Kalman Filter bank" block in Figure 4.6, using the data generated for the horizontal model.

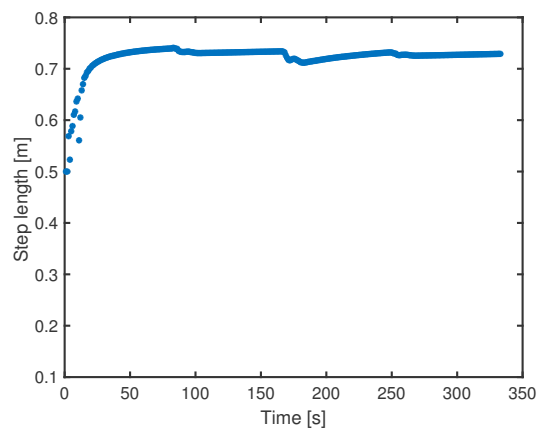

(a) Estimated step length by mixing hypotheses for the thresholds.

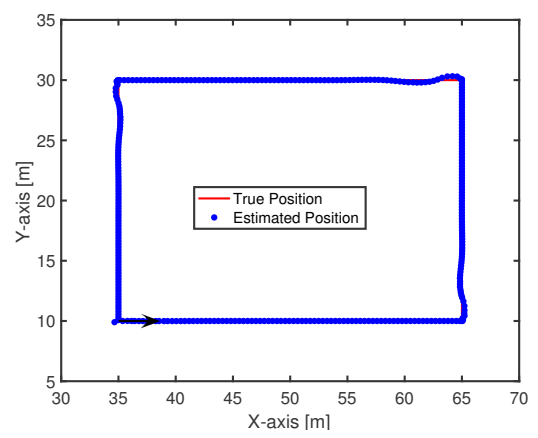

(b) Estimated position against the true position.

Figure 4.10: The results of the "output estimation calculation" block in Figure 4.6, using the data generated for the horizontal model.

\subsubsection{Data Collection}

Several candidates with different weights and heights participated in the data collection experiment. For each candidate, different motion modes consisting of walking (W), running (R), and standing still (SS) are considered. The candidates followed two trajectories, each with three or four sharp turns, while holding a smartphone fixed in hand in front of them. The Sensor Fusion Android app [39, 60] was used to log the measurements from GPS, gyroscope and accelerometer 
Table 4.2: Measurement Scenarios

\begin{tabular}{|c|l|l|l|}
\hline Scenario & Device mode & \multicolumn{1}{|c|}{ Motion mode } & Location \\
\hline Liu1 & Fixed in hand & Walking (W) & Outdoor \\
\hline Liu2 & Fixed in hand & $\begin{array}{l}\text { Walking (W) } \\
\text { Standing Still (SS) } \\
\text { Running (R) }\end{array}$ & Outdoor \\
\hline
\end{tabular}

sensors.

In this study, two different scenarios are considered to evaluate the proposed methods. A summary of the scenarios is given in Table 4.2.

\section{Offline Kalman Filter Bank Results}

Table 4.3 presents the results obtained from applying the longitudinal model described in Section. 4.1.1 to the collected data. The elements of the process noise covariance matrix for this model were selected as $\sigma_{a}^{2}=10\left(\mathrm{~m} / \mathrm{s}^{2}\right)^{2}$ and $\sigma_{L}^{2}=0.002 \mathrm{~m}^{2}$, and the measurement noise variances for GPS and step length are $\sigma_{\mathrm{v}, \mathrm{GPS}}^{2}=9(\mathrm{~m} / \mathrm{s})^{2}$ and $\sigma_{\text {step }}^{2}=0.04 \mathrm{~m}^{2}$, respectively.

The results of adaptively determining the step detection threshold are compared to the results when the threshold is fixed such that it suits user 1 . In Table 4.3, the estimates are compared to the ground truth values for the gait parameters and the traveled distances. As Table 4.3 suggests, the estimates obtained from using the fixed threshold for user 1 are comparable to those obtained with the adaptive scheme. However, the fixed threshold does not perform well for the second user. In that case, choosing a too large step detection threshold results in too few steps being detected. Hence, the threshold that suits user 1 works less well with user 2.

Next, we evaluate the performance of the horizontal model using the real data. The elements of the process noise covariance matrix for the horizontal model were selected as $\sigma_{a}^{2}=10, \sigma_{\alpha}^{2}=0.5$, and $\sigma_{L}^{2}=0.002$ and the measurement noise variance for GPS was selected as $\sigma_{\mathrm{p}, \mathrm{GPS}}^{2}=9$, for gyroscope as $\sigma_{\text {gyro }}^{2}=0.64$, and for the step length as $\sigma_{\text {step }}^{2}=0.04$.

Figure 4.11 presents the results obtained from using the horizontal model in which the PDR estimation is shown with adaptive and fixed gait step detection thresholds (again chosen to suit user 1 the best) when two users walked slowly. The threshold for step detection has been estimated for Figure 4.11a and Figure 4.11c. Conversely, in Figure 4.11e the fixed threshold is used and applied to the same measurement set as used in Figure 4.11c. The GPS signal (green dots) is only assumed available for a few seconds in the beginning and middle of experiments.

Using the adaptive step detection threshold, as shown in Figure 4.11, provides reasonably good position estimates even during periods without GPS coverage.

At the same time, Figure 4.11e shows how large influence poorly chosen gait 
Table 4.3: Estimated gait parameters and travelled distance versus true one [48].

\begin{tabular}{|c|c|c|c|c|}
\hline & $\begin{array}{c}\text { Traveled } \\
\text { distance } \\
{[\mathrm{m}]}\end{array}$ & $\begin{array}{c}\text { Number } \\
\text { Of } \\
\text { Steps }\end{array}$ & $\begin{array}{c}\text { Position } \\
\text { error at the } \\
\text { end point } \\
{[\mathrm{m}]}\end{array}$ & $\begin{array}{c}\text { Threshold } \\
{\left[\mathrm{m} / \mathrm{s}^{2}\right]}\end{array}$ \\
\hline $\begin{array}{c}\text { Ground Truth } \\
\text { User } 1\end{array}$ & 248 & 333 & 0 & 2.06 \\
\hline $\begin{array}{l}\text { User 1 with } \\
\text { adaptive } \\
\text { gait parameters }\end{array}$ & 246.1 & 329 & 6.9 & 2 \\
\hline $\begin{array}{l}\text { User } 1 \text { with } \\
\text { fixed } \\
\text { gait parameters }\end{array}$ & 245.2 & 321 & 8.6 & 4 \\
\hline $\begin{array}{c}\text { Ground Truth } \\
\text { User } 2\end{array}$ & 242 & 346 & 0 & 0.81 \\
\hline $\begin{array}{c}\text { User } 2 \text { with } \\
\text { adaptive } \\
\text { gait parameters }\end{array}$ & 240.1 & 352 & 9.8 & 0.8 \\
\hline $\begin{array}{l}\text { User } 2 \text { with } \\
\text { fixed } \\
\text { gait parameters }\end{array}$ & 227.7 & 141 & 32.8 & 2 \\
\hline
\end{tabular}

parameters can have on the end result, when compared to adaptively chosen parameters. It should be noted that in these limited experiments, simply choosing a very low step detection threshold would improve the result. However, we anticipate this not to be the case in more realistic settings with uneven gait and regular stops which motivates the choice of parameters here.

The step lengths estimated with adaptive threshold, presented in Figure $4.11 \mathrm{~d}$ and Figure $4.11 \mathrm{~b}$, are fairly constant. In this figure, green lines indicate availability of the GPS signal, as in the previous case. Figure $4.11 \mathrm{f}$ shows the negative effect on the step length when the steps are incorrectly detected due to a poor step detection threshold. 


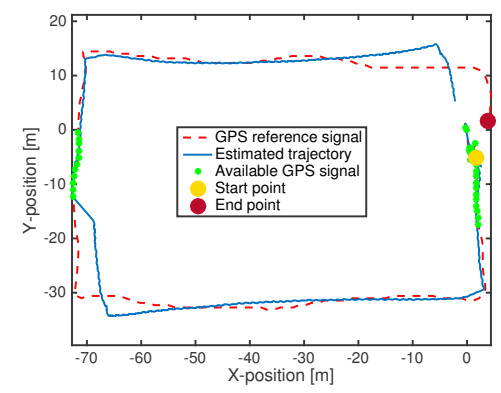

(a) User 1 with adaptive parameters.

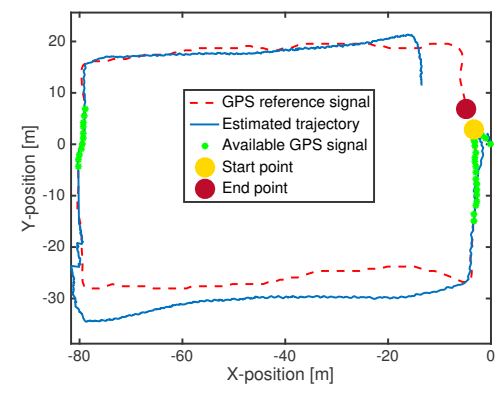

(c) User 2 with adaptive parameters.

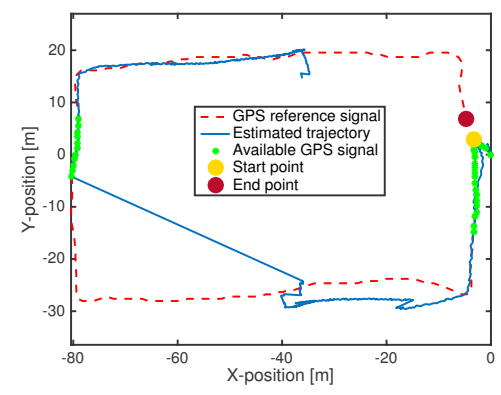

(e) User 2 with fixed parameters.

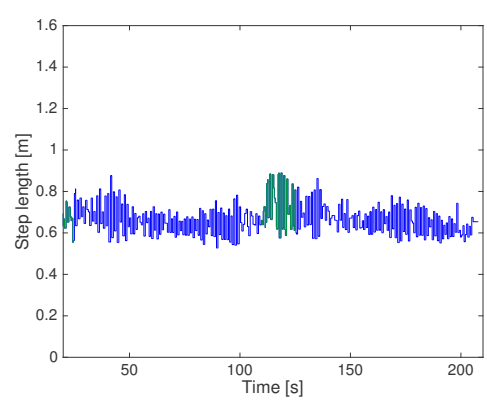

(b) The estimated step lengths, the green lines indicate time slots of available GPS signals.

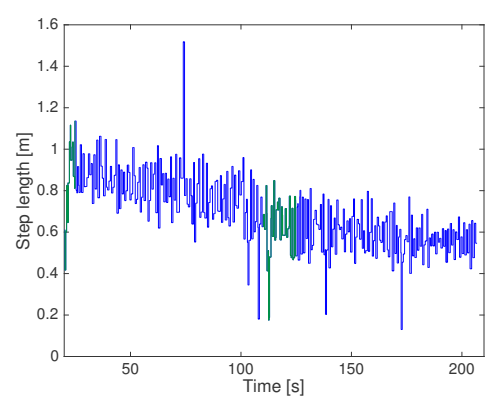

(d) The estimated step lengths, the green lines indicate time slots of available GPS signals.

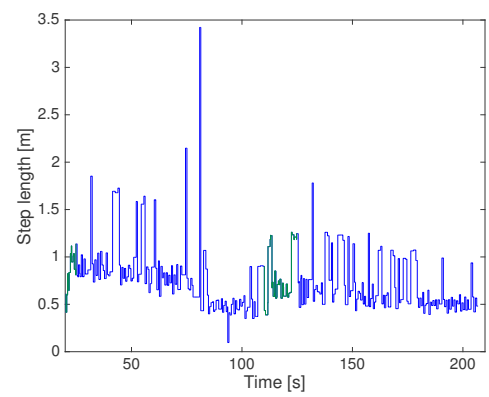

(f) The estimated step lengths, the green lines indicate time slots of available GPS signals.

Figure 4.11: Illustration of the estimated trajectory with PDR and GPS reference trajectory. The green dots indicate where GPS signals were assumed available. The user starts walking south and in total makes four sharp turns until the end point is reached. The users walked slowly and with constant speed during the data collection [48, C2016 IEEE]. 


\section{Online Kalman Filter Bank Results}

The online Kalman filter bank results are presented in this section. The results of the longitudinal model are gathered for different walking modes; in the simplest scenario, the user walks with constant speed during the whole measurement, while complex scenarios mimic a more realistic application in which users change their motion mode between walking, standing still, and running. In the following figures, different modes are distinguished by their initial letter; "W" stands for walking, "SS" for standing still and " $\mathrm{R}$ " for running. The process noise covariance matrix for this model is $Q=\operatorname{diag}([10,0.00000001])$ and the measurement noises standard deviations, for the GPS and step length are selected as before. The TPM matrix is a $3 \times 3$ identity matrix since three hypotheses are used in this scenario.

The results obtained for the simple walking mode are summarized in Figure 4.12 . Figure $4.12 \mathrm{a}$ and Figure $4.12 \mathrm{~b}$ represent the mode probability with and without GPS signal for three different thresholds. According to these figures, the mode probability for the second threshold has the highest value that is very well in-line with our expectations compared to the other two thresholds that stand for extreme low and high values.

The results of the estimated step lengths for these two situations (with GPS as well as the GPS denied conditions), are illustrated in Figure 4.12c and Figure $4.12 \mathrm{~d}$, respectively. The step length estimate remains constant for all measurements in both situations, as expected since the user is always walking with constant speed throughout the whole experiment. The step lengths are estimated to be around $0.8 \mathrm{~m}$ which is very reasonable for a normal person.

The traveled distance estimation error for both environments is computed and illustrated using a cumulative distribution function (CDF) in Figure 4.12e and Figure 4.12f. As the figures indicate, the traveled distance is estimated with rather a low error, even when the GPS is not always available. The ground truth is assumed to be the position measurements provided by the GPS. 


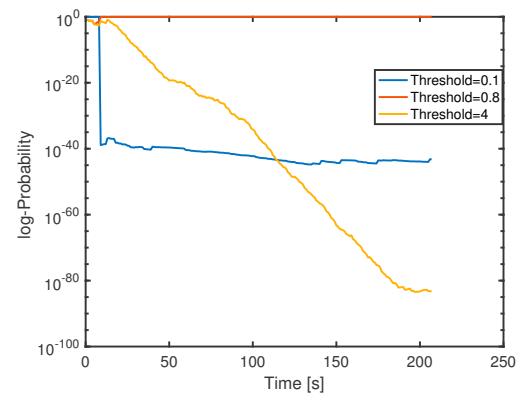

(a) Mode probability for three different thresholds. GPS is available during entire experiment.

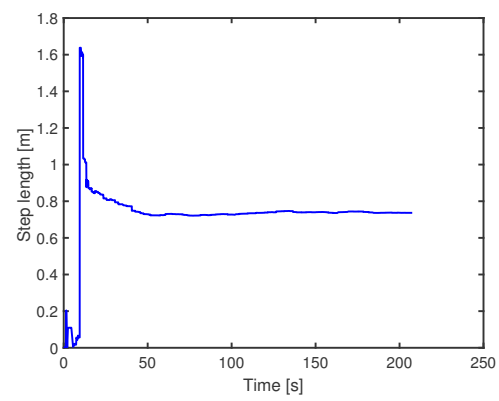

(c) The estimated step length. GPS is available during entire experiment.

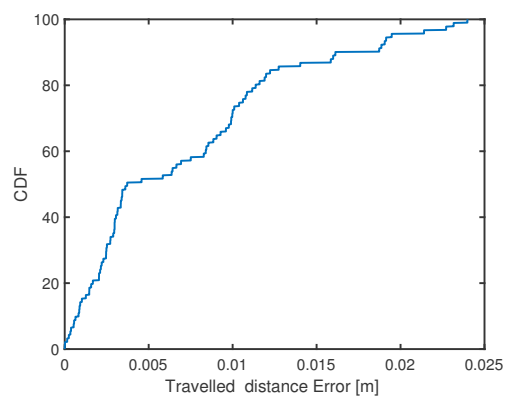

(e) The CDF of root mean square error for estimated travelled distance. GPS is available during entire experiment.

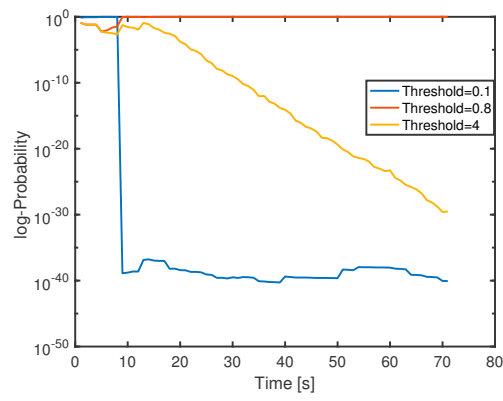

(b) Mode probability for three different thresholds in GPS denied environment.

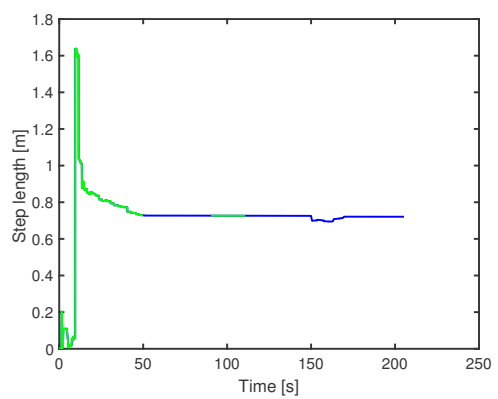

(d) The estimated step length in GPS denied environment. Green solid line indicate where GPS signals were assumed available.

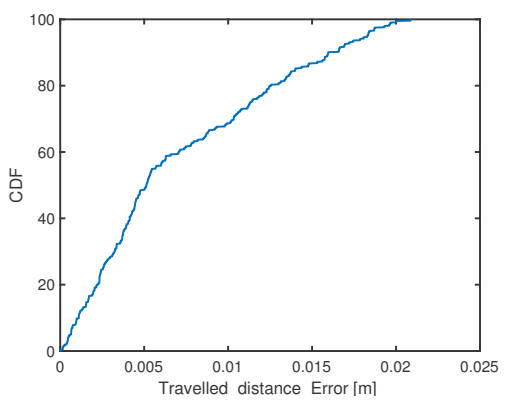

(f) The CDF of root mean square error for estimated travelled distance in GPS denied environment.

Figure 4.12: Results for longitudinal model with real data for simple walking pattern. 


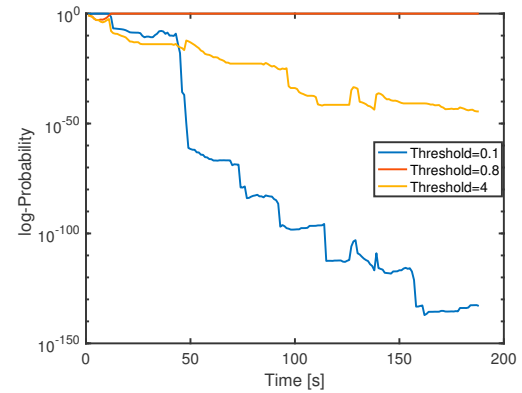

(a) Mode probability for three different thresholds. GPS is available during entire experiment.

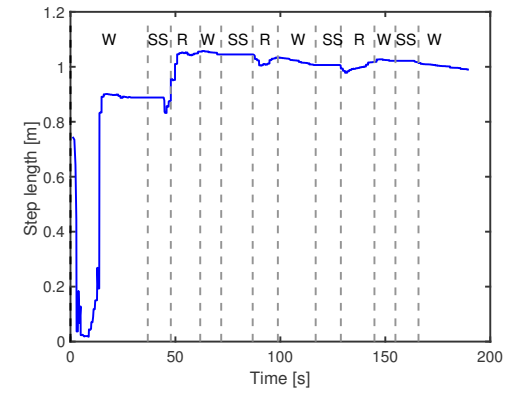

(c) The estimated step length. GPS is available during entire experiment.

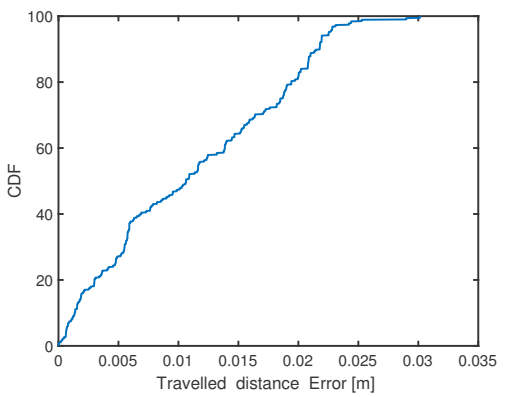

(e) The CDF of root mean square error for estimated travelled distance with GPS signal always available during entire experiment.

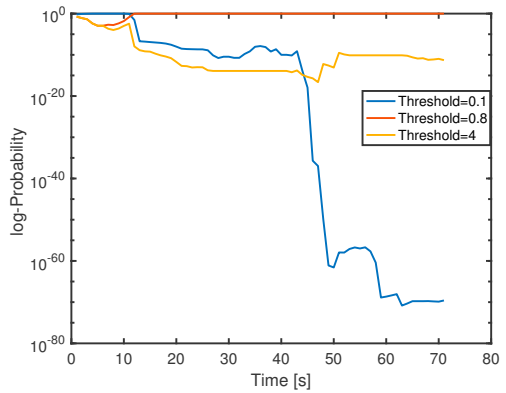

(b) Mode probability for three different thresholds in GPS denied environment.

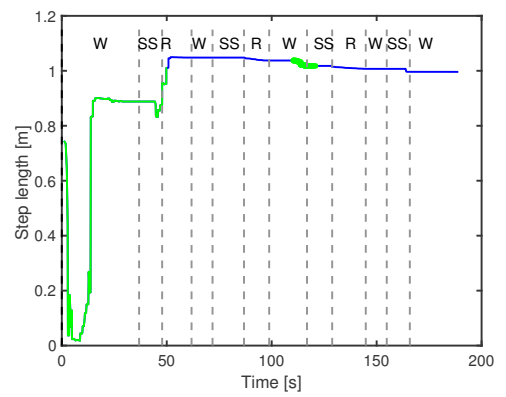

(d) The estimated step length in GPS denied environment. Green solid line indicate where GPS signals were assumed available.

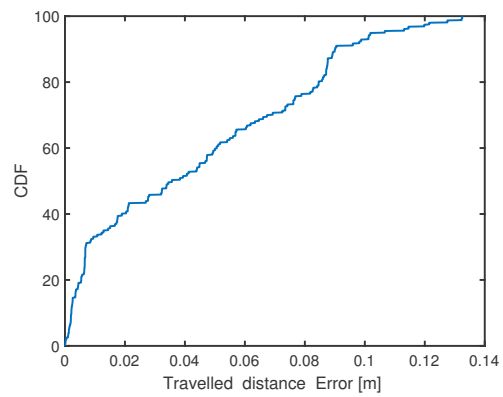

(f) The CDF of root mean square error for estimated travelled distance in GPS denied environment.

Figure 4.13: Results for longitudinal model with real data for complex walking pattern. 
The complex walking pattern in the longitudinal model is also evaluated and the results are reported in Fig 4.13. Similar conclusions as in the simple walking mode are valid in these scenarios as well. As these figures suggest, the PDR with gait parameter learning results in good estimation for step length and traveled distance even during periods without GPS coverage.

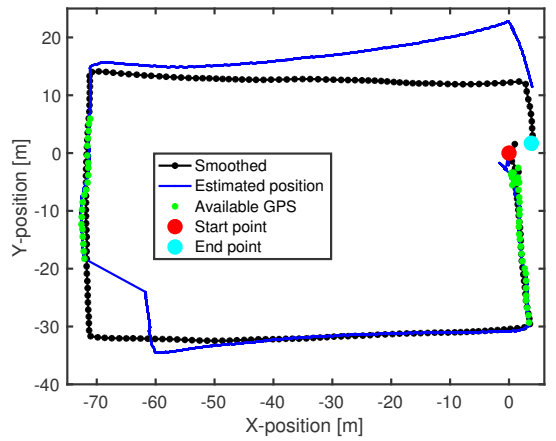

(a) Estimated trajectory with PDR. Only walking is performed by user.

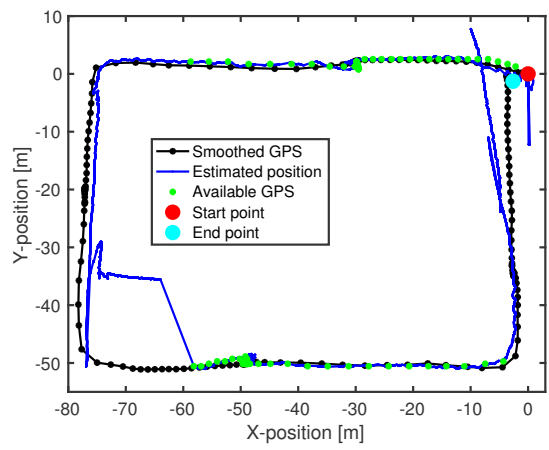

(c) Estimated trajectory with PDR. Different motion modes $(W, R, S S)$ are performed by the user.

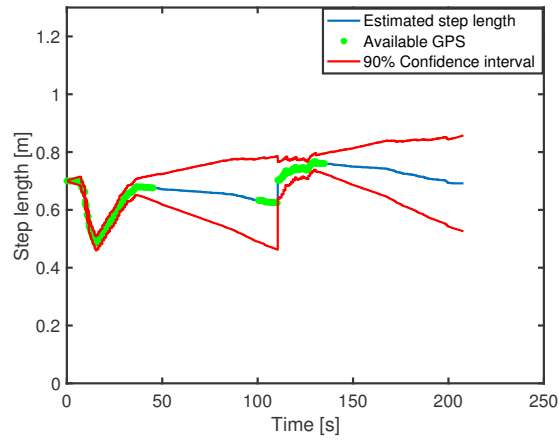

(b) Estimated step length with PDR. Only walking is performed by user.

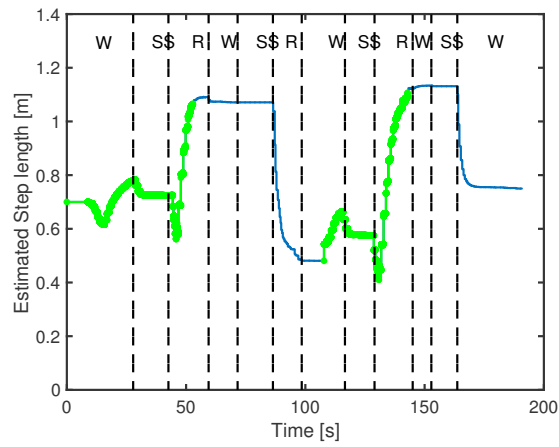

(d) Estimated step length with PDR. Different motion modes $(W, R, S S)$ are performed by the user.

Figure 4.14: Illustration of the estimated results obtained by "Model 1" proposed in Section 4.1.2, against the smoothed GPS as the reference trajectory. Green dots indicate time instances at which GPS signals are available. 
The results for the horizontal model are presented in both simple and complex walking patterns. The elements of the process noise covariance matrix for both horizontal models were selected as $\sigma_{a}^{2}=10\left(\mathrm{~m} / \mathrm{s}^{2}\right)^{2}, \sigma_{\alpha}^{2}=10 \mathrm{rad}^{2}$ and $\sigma_{L}^{2}=1 e-$ $5 \mathrm{~m}^{2}$.

\section{Model 1}

Figure 4.14 presents the results for "Liu1" and "Liu2" scenario using "model 1 " proposed in Section 4.1.2. In this figure, the step length gradually decreases after the GPS is switched off. This causes large position estimation errors. For "Liu2" scenario, in the intervals where GPS update is unavailable and no steps are detected from the accelerometer signal, filters perform the prediction phase only. This results in the case in which the user is mistakingly assumed to be walking forward with the same speed and step length. Large position estimation errors are hence obtained in this scenario using "model 1".

\section{Model 2}

"Model 2" is proposed in Section 4.1.1 to improve the step length estimation performance. In this model, the step length is independent of the speed and is computed based on the previous estimated position. In this model, the states are extended with previous estimated position (when the step is detected) and will be used when the new step is detected to estimate the step length. Figure 4.15 presents results of this approach for "liu1" scenario. As the figure suggests, the estimations are improved and the decreasing trend of the step length has a much lower slope compared to the previous model. However, the result of position estimation for "liu2" scenario still contains large error.

\subsubsection{Standing Still Detection}

To improve the performance of position estimation for the scenarios containing standing still, an algorithm has been implemented to detect the standing still modes. For this purpose, two constants are defined to check if either GPS is available or a step is detected.

The GPS signals are usually received every second, hence the GPS constant is set to 1.6 second to have large enough margin in case the signal is received with some delay. For step detection, depending on the motion mode, the value varies between 0.5 to 0.7 second for walking and running modes, respectively. Here, the constant is set to 0.8 second. If the difference between the current received measurement time and the previous GPS time and step time exceeds the defined limit, it is assumed that neither GPS signal is received nor step is detected. This implies that the subject is in standing still mode.

In cases where the standing still mode is detected, the values for the speed and the step length are set to zero and the prediction phase in the filter will only update two states, heading and angular velocity. As soon as a new step is detected or GPS signal becomes available, the speed and step length will be set 


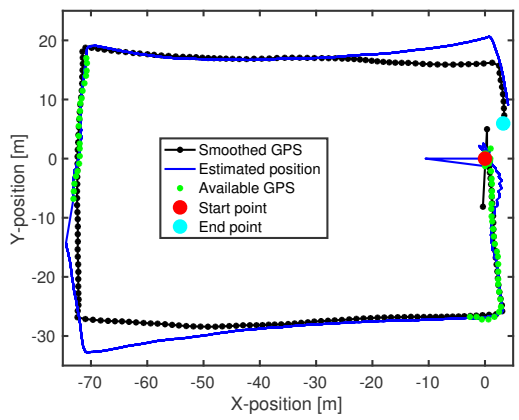

(a) Estimated trajectory with PDR. Only walking is performed by user.

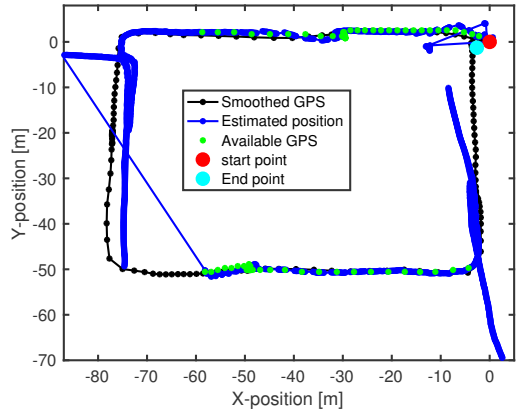

(c) Estimated trajectory with PDR. Different motion modes $(W, R, S S)$ are performed by the user.

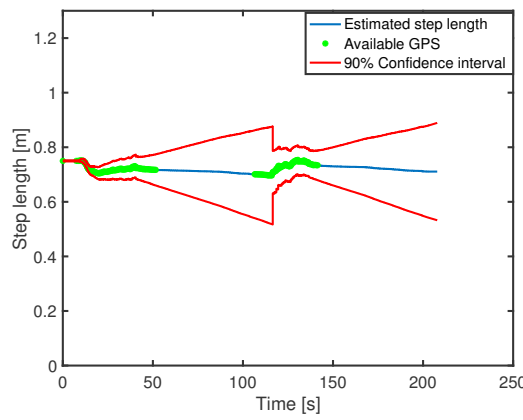

(b) Estimated step length with PDR. Only walking is performed by user.

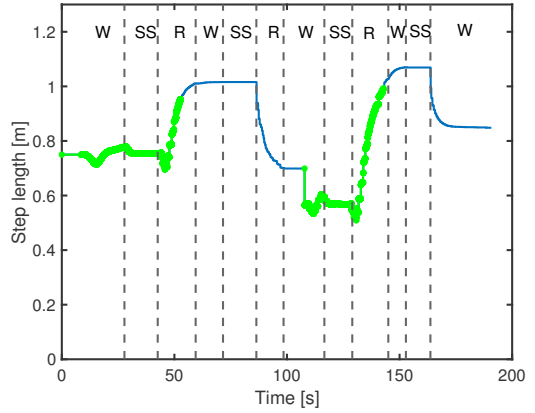

(d) Estimated step length with PDR. Different motion modes $(W, R, S S)$ are performed by the user.

Figure 4.15: Illustration of the estimated results obtained by "Model 2" proposed in Section 4.1.2, against the smoothed GPS as the reference trajectory. Green dots indicate time instances at which GPS signals are available.

to the last value before standing still mode and prediction will apply for all state components.

Figure 4.16 represents the performance of the PDR estimation using standing still detection algorithm. In these figures, the estimated positions are improved and follow the trajectory. However, there is a drift in the last part of the trajectory. GPS signal is disconnected at point $(-18,-50) \mathrm{m}$ where the user was in the running mode. The speed remains the same for the rest of the measurement and the step length is estimated around $1.1 \mathrm{~m}$ which corresponds to the running mode rather than walking. However, since the user was actually walking in that interval, until the end point is reached, the position estimation will contain some errors at the end of the trajectory.

It is worth mentioning that by using standing still detection algorithm both 


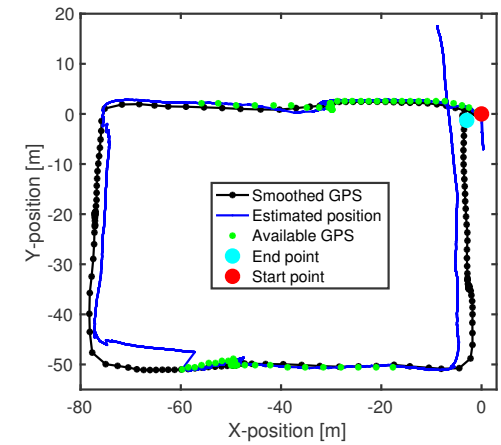

(a) Estimated trajectory with PDR. "Model 1"

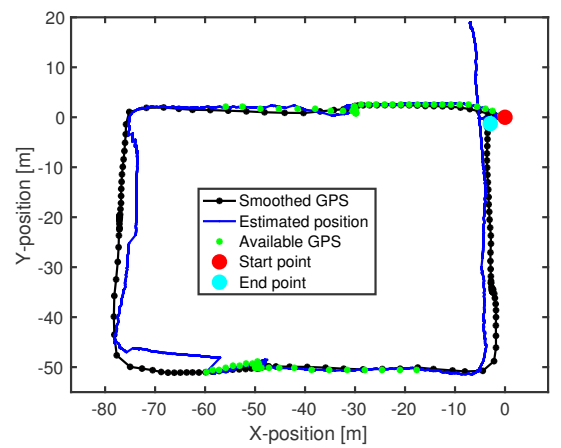

(c) Estimated trajectory with PDR. "Model 2"

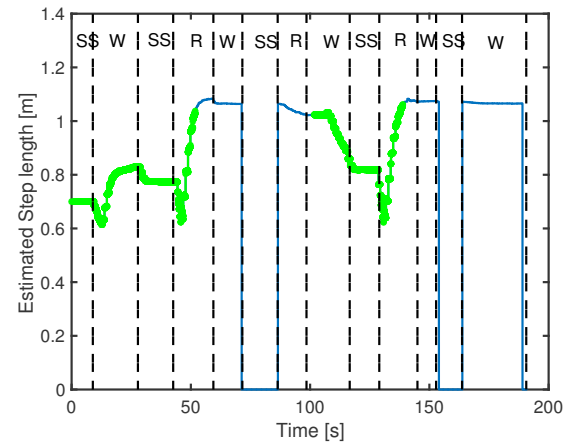

(b) Estimated step length with PDR. "Model 1"

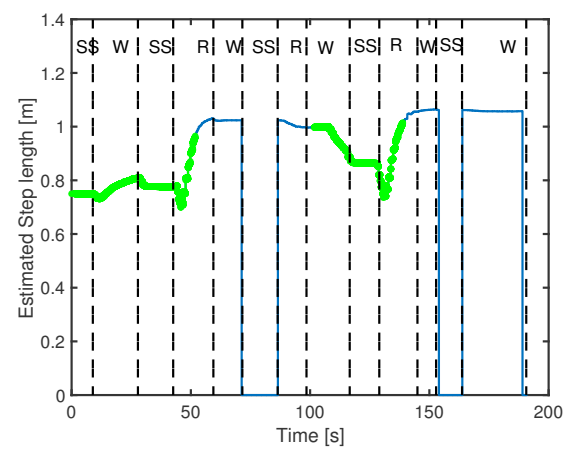

(d) Estimated step length with PDR. "Model 2"

Figure 4.16: Illustration of the estimated results obtained by "Model 1" and "Model 2" using standing still detector against the smoothed GPS as the reference trajectory. Green dots indicate time instances at which GPS signals are available.

proposed horizontal models have almost similar performance, though it can be seen in step length estimation that "model 2" has slightly better performance.

\subsubsection{Motion Mode Classification Using HMM}

Using standing still detection improved the estimated position accuracy. However, the existing drift in the estimates made some room for further improvements. As mentioned earlier, in standing still detection algorithm the last speed before standing still mode was stored. Irrespective of the user's motion after the standing period, the stored value was then used as soon as a step was detected. As Figure 4.16 suggests, this approach negatively affects the estimates. 


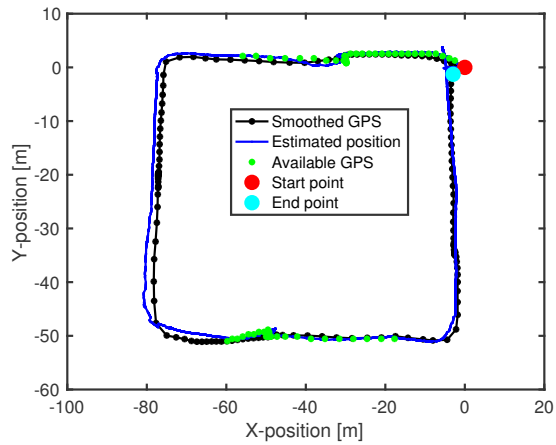

(a) Estimated trajectory with PDR.

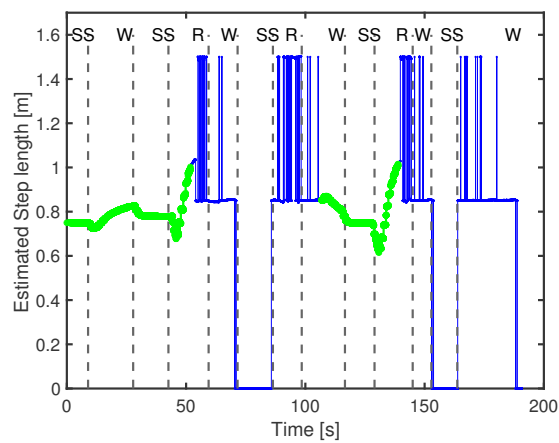

(b) Estimated step length with PDR.

Figure 4.17: Illustration of the estimated results obtained by "Model 1" proposed in Section 4.1.2, against the smoothed GPS as the reference trajectory using motion mode classification. Green dots indicate time instances at which GPS signals are available. Different motion modes $(W, R, S S)$ are performed by the user.

In order to improve the algorithm, a motion mode classification has been applied to the data while the GPS is not available. The step length and speed will be adjusted properly by the motion mode detection. Figure 4.17 represents the performance of the PDR estimation using motion mode classification. In these figures, the estimated positions are improved and follow the trajectory and the drifting issue is resolved. However, due to some miss-classification, the step length estimation is jumping between running mode and walking mode.

\subsection{Conclusion}

Inertial measurements are another source of information that can be used either in standalone positioning algorithms or together with other radio measurements. To mitigate the main disadvantage of the standalone INS systems, being the integration drift caused by integrating inertial sensors measurements, a framework for a pedestrian dead reckoning algorithm was developed.

In this application, we proposed two extended PDR models in Section 4.1.1 which were the extended version of basic PDR, given in Section 4.1. The approach was based on an offline and online multi-rate Kalman filter bank that learned gait parameters in time intervals when GPS position estimates were available. This improved the PDR in time intervals when the measurement is unavailable. The proposed methods had been evaluated using simulated data as well as real experimental IMU measurements, presented in Section 4.4 where it was shown that promising results could be obtained especially when the step detection threshold was chosen adaptively.

In the offline approach, the algorithm assumed a subject moving with con- 
stant speed. It is explained that the results deteriorate if a fixed threshold is used that is not appropriately chosen to suit the current user.

To relax the constant velocity assumption, the online approach set no restriction on the subjects speed and the experiment was done while different walking models were considered; running, walking and standing still. However, it was shown in the online Kalman filter bank in Section 4.4 .2 that by having more variations in the user's walking patterns and with regular stops or running, the step detection and position estimation accuracy were affected. 


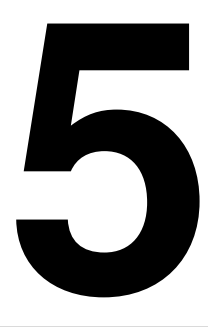

\section{IMU Dataset For Motion and Device Mode}

This work is presented in:

P. Kasebzadeh, G. Hendeby, C. Fritsche, F. Gunnarsson, F. Gustafsson. IMU Dataset For Motion and Device Mode Classification. In Proceedings of the 8th International Conference on Indoor positioning and indoor navigation, Sapporo, Japan, September 2017.

In this chapter, we study human motion and device mode classification. Classification of motion mode (walking, running, standing still) and device mode (hand-held, in pocket, in backpack) is an enabler in PNS for the purpose of design parameter settings.

Our main contribution is to make available one of the most extensive datasets for this problem, including inertial data from eight users, each one performing three pre-defined trajectories carrying four smartphones and seventeen inertial measurement units on the body. All kind of metadata is available such as the ground truth of all modes and position. A second contribution is the first study on a joint classifier of motion and device mode, respectively, where preliminary but promising results are presented.

The rest of the chapter is organized as follows: Section 5.1 explains experimental setup in detail, followed by a description of available data in Section 5.2. In Section 5.3 data analysis and initial classification results are presented.

\subsection{Experiment Setup}

Our investigation is based on an extensive experimental study where different users repeated the same trajectories and sequences of modes. We logged data using low cost sensors including acceleration, angular rate, magnetic field, barometric pressure and also GPS as a position reference. Data were measured by using 
four smartphones and 17 IMUs configured in a body suit. The IMUs generate data of somewhat higher accuracy than the smartphones, and the body suit software makes use of advanced biomechanical models to provide accurate description of true motion of all body parts, which in turn could be used to simulate data from any other part of the body. Ground truth of the device and motion mode is available from the experimental setup. Table 5.1 summarizes different device and motion modes. In order to simplify referring to each of these scenarios, Table 5.1 also assigns a specific class to each of them. We believe this to be one of the most extensive datasets publicly available for PNS.

Table 5.1: Motion-Device mode Classification [49].

\begin{tabular}{|c|c|c|c|}
\hline Device mode $\quad \begin{array}{c}\text { Motion } \\
\text { mode }\end{array}$ & $\begin{array}{l}\text { Standing Still } \\
\text { (SS) }\end{array}$ & $\begin{array}{c}\text { Walking } \\
\text { (W) }\end{array}$ & $\begin{array}{l}\text { Running } \\
\text { (R) }\end{array}$ \\
\hline Fixed hand (1) & \multirow{4}{*}{ Class SS } & Class W1 & Class R1 \\
\hline Swinging hand (2) & & Class W2 & Class R2 \\
\hline Pocket (3) & & Class W3 & Class R3 \\
\hline Backpack (4) & & Class W4 & Class R4 \\
\hline
\end{tabular}

In this section, we first introduce the hardware used in the experiments, then all measurement scenarios are described in detail. Finally, characteristics of all participants are presented to give a better comparison on signal behavior for different subjects with different attributes.

\subsubsection{Sensors}

The hardware can be grouped into two categories; high and low quality; MVN ${ }^{1}$ and Nexus 5, respectively. Subsequently, the signals from the mobile phones will be compared with the MVN system to have a better classification for mobile signals.

\section{Xsens MVN Motion Capture}

The Xsens MVN system has been used to capture the whole body motion. In the experiments the "MVN Awinda" system has been used [99]. It contains 17 wireless Motion Trackers (MTw), an Awinda station, and MTw full body velcro straps.

The MTw is a miniature inertial measurement unit containing a 3-D linear accelerometer, a 3-D rate gyroscope, a 3-D magnetometer, and a barometer.

The 17 trackers are placed at strategic locations on the body (secured by straps), to measure motion of each body segment. Figure 5.1 shows the location of the straps and attached MTw. The MVN system is controlled by the MVN studio software. A snapshot of the MVN system is shown in Figure 5.2.

\footnotetext{
$1_{\text {https://www.xsens.com/products/xsens-mvn-analyze/ }}$
} 


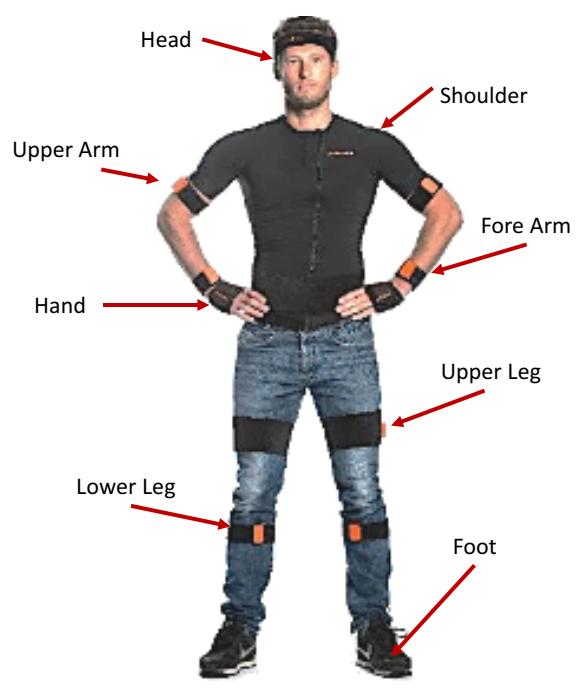

Figure 5.1: MVN Awinda Straps by courtesy of Xsens [49, C2017 IEEE].

\section{Nexus 5}

Four Nexus 5 smartphones are carried by the subjects in the experiments. The Nexus 5 is equipped with multiple sensors; A-GPS, the 3-D linear accelerometer, the 3 -D rate gyroscope, and the barometer are the sensors that considered in this work.

The Sensor Fusion Android app [40, 60], installed on the Nexus 5 phones, is used to log the sensors measurements from the phones.

All phone locations used in the experiment are presented in Table 5.1. A summary of the details about both the MVN system and the phone is provided in Table 5.2; application, sampling frequency, sensor's positions, and IMU sensors.

\subsubsection{Scenarios}

Measurements were collected in a building at Twente university. During the experiments the subjects walked three different paths, with a mixture of different motion modes, as represented in Table 5.1. We followed certain rules when gathering the data so that the obtained measurements should mimic reality. For instance, to avoid any abnormal behavior the subjects were asked to carry one smartphone in the hand at each time. One run-through of the scenarios including a preparation phase lasts around 45-60 min.

Figure 5.3 illustrates three different paths on top of the map of the area where measurements were obtained. The paths on the maps are for illustrative purposes and are inexact. For each path in Figure 5.3, the subject holds one mobile phone in the hand, two more phones in front and back pockets, and one in the backpack. In this section, we thoroughly present the three measurement scenarios studied 


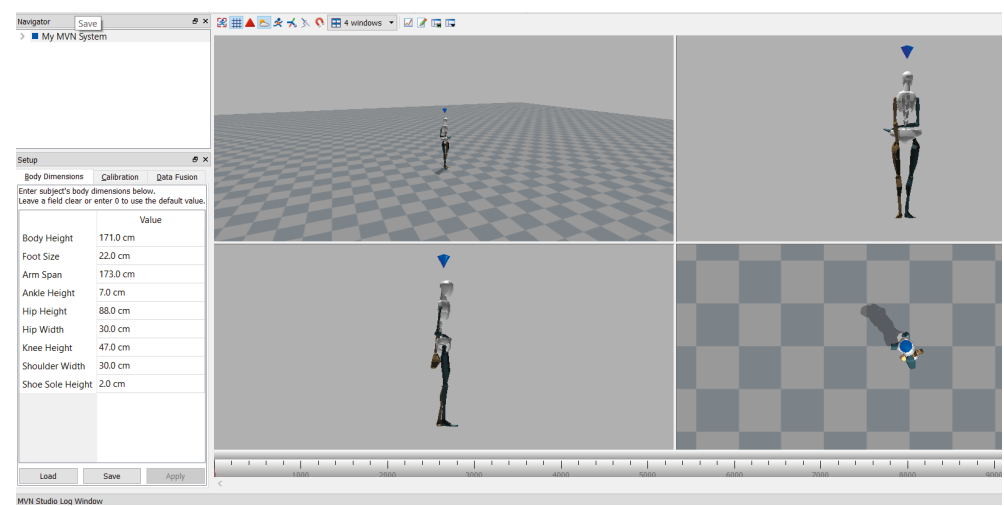

Figure 5.2: MVN Studio BIOMECH application [49, C2017 IEEE].

Table 5.2: Measurement device specifications [49].

\begin{tabular}{|c|c|c|c|c|}
\hline Device & Application & $\begin{array}{c}\text { Sampling } \\
\text { Frequency } \\
{[\text { Hz] }}\end{array}$ & Position & Sensors \\
\hline Xsens & Awinda & 60 & $\begin{array}{c}\text { Head } \\
\text { Shoulders } \\
\text { Upper Arms } \\
\text { Fore Arms } \\
\text { Upper Legs } \\
\text { Lower Legs } \\
\text { Hands } \\
\text { Feet } \\
\text { Pelvis }\end{array}$ & $\begin{array}{c}\text { Accelerometer } \\
\text { Gyroscope } \\
\text { Barometer }\end{array}$ \\
\hline Nexus 5 & $\begin{array}{c}\text { Sensor } \\
\text { Fusion } \\
\text { app }\end{array}$ & 100 & $\begin{array}{c}\text { Front Pocket } \\
\text { Back Pocket } \\
\text { Backpack }\end{array}$ & $\begin{array}{c}\text { Accelerometer } \\
\text { Gyroscope } \\
\text { Magnetometer } \\
\text { Barometer }\end{array}$ \\
\hline
\end{tabular}

in this work. One scenario contains outdoor-only measurements, while the other two scenarios combine both outdoor and indoor paths. All three scenarios share the property that the measurement begins and ends at the same point, in an outdoor environment. 


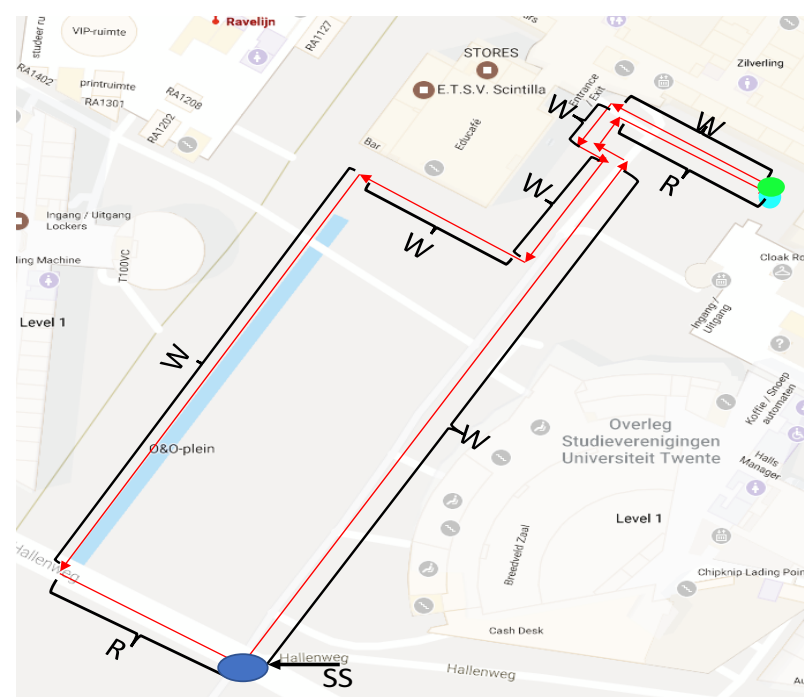

(a) Outdoor-only scenario with all classes (Case 1).

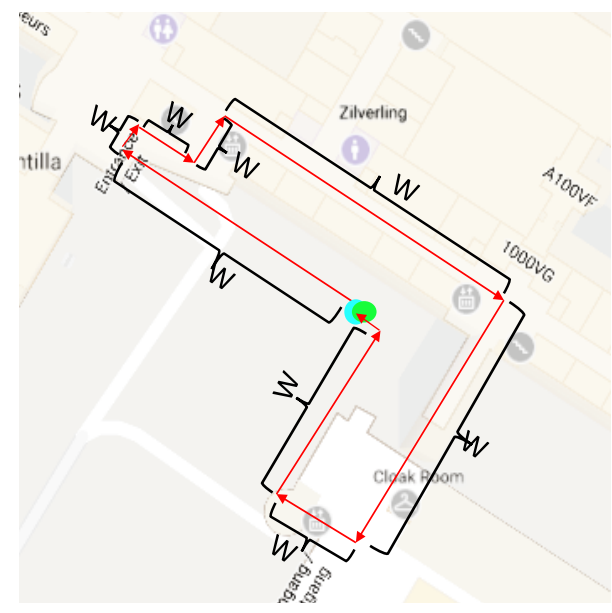

(b) Outdoor-Indoor scenario containing the $W 1$ class (Case 2).

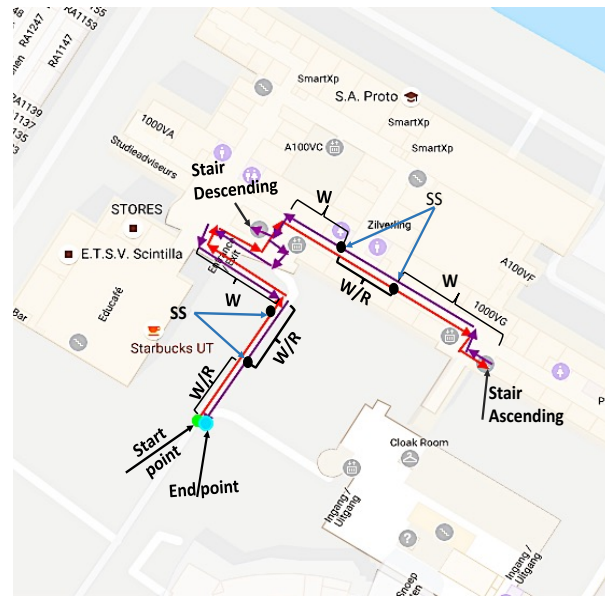

(c) Outdoor-Indoor scenario containing all classes. Forward and backward paths are indicated with red and purple colors, respectively (Case 3 and Case 4).

Figure 5.3: Different measurement scenarios. The start and end points are indicated by green and cyan dots, respectively. All the motion modes and classes are defined based on Table 5.1 [49, (C2017 IEEE].

\section{Outdoor-only, Standing Still, Walking and Running}

The simplest scenario corresponds to Figure 5.3a where the whole measurement is performed outdoors where the GPS signal is available. In this scenario, measure- 
Table 5.3: Measurement scenarios. Device and motion modes are presented in Table 5.1. Paths corresponding to each case are depicted in Figure 5.3 [49].

\begin{tabular}{|c|c|c|c|c|}
\hline Scenario & $\begin{array}{c}\text { Device } \\
\text { Mode }\end{array}$ & $\begin{array}{c}\text { Motion } \\
\text { Mode }\end{array}$ & Participants & $\begin{array}{c}\text { Duration } \\
\text { average) } \\
{[\mathbf{s}]}\end{array}$ \\
\hline Case 1 & $1,2,3,4$ & W,R,SS & $\begin{array}{c}\text { 5 Males } \\
\text { 2 Females }\end{array}$ & 190 \\
\hline Case 2 & $1,3,4$ & $\mathrm{~W}$ & $\begin{array}{c}6 \text { Males } \\
\text { 2 Females }\end{array}$ & 200 \\
\hline Case 3 & $\begin{array}{c}1,3,4 ; \\
2,3,4\end{array}$ & $\begin{array}{c}\mathrm{W} \\
\text { Upstairs } \\
\text { Downstairs }\end{array}$ & $\begin{array}{c}\text { 6 Males } \\
\text { 2 Female }\end{array}$ & 280 \\
\hline Case 4 & $\begin{array}{c}1,3,4 ; \\
2,3,4\end{array}$ & $\begin{array}{c}\text { W,R,SS } \\
\text { Upstairs } \\
\text { Downstairs }\end{array}$ & $\begin{array}{c}\text { 6 Males } \\
\text { 2 Female }\end{array}$ & 270 \\
\hline
\end{tabular}

ments are performed while the subject covers several motion and device modes, corresponding to Case 1 in Table 5.3.

\section{Outdoor-indoor, Walking}

As in the previous scenario, the measurement starts and ends at the same point outside the building. However, a bit in to the track, the subject gets into the building and walks across a corridor, as illustrated in Figure 5.3b. In this scenario, measurements are performed while the subject walks the whole path and holds the smartphone flat and fixed in the hand, corresponding to Case 2 in Table 5.3.

\section{Outdoor-indoor, Standing Still, Walking and Running}

In this scenario, we consider the most complex behavior in terms of motion modes and also the experiment path. Along the path depicted in Figure 5.3c, the subject starts outside the building, then gets into the building and passes one corridor, takes the stairs up and passes a certain distance, followed by taking the stairs down and getting back to the starting point. The designed path in this scenario, is measured for two different cases:

- Case 3: The subject walks along the path for both sets of device modes presented in Table 5.3 (each set is performed separately).

- Case 4: The subject has several motion modes along the path for both sets of device modes presented in Table 5.3 (each set is performed separately).

\subsubsection{Participants}

The described experiments were performed by twelve volunteers, 7 males and 5 females, with ages ranging from 25 to 45 years old. Due to some technical 
and practical issues (GPS signal loss outdoors, physical difficulties making it impossible for subjects to perform all the experiments), only 6 men and 2 women performed all the explained scenarios.

\subsection{Available Data}

This section provides a detailed explanation of how the data was collected followed by a description of the ground truth. Finally, we clarify the data structure as well as provide instructions on accessing different parts of the available data summarized in Table 5.3.

\subsubsection{Collected Data}

Data is gathered from the scenarios in Section 5.1.2 using the hardware specified in Section 5.1.1. Before each experiment/data collection, the MVN Awinda is calibrated. The calibration can be done by MVN studio in a less disturbed magnetic field area while the subject is standing in a fixed pose for around 5 seconds. Table 5.3 summarizes all the different scenarios. It is worth noting that the laptop connected to the Awinda station must be near the subjects while recording measurements due to short signal range. Figure 5.4 shows a subject while doing an experiment. She is wearing Xsens suit and carrying several phones.

The available data for both smartphone and Xsens suits together with units of each measurement are presented in Table 5.4. Both the raw sensory data as well as the virtual IMU measurements from the Biomechanical (BM) model are extracted from MVN studio and provided in the dataset. Although MTws are equipped with a barometer, the MVN studio cannot extract this feature, therefore, the MTw data for the barometer is not used.

The phone data is only available in raw version. The GPS signal from the phones is converted to ENU coordinates, with the first measured GPS position considered as the reference point.

\subsubsection{Ground Truth}

The ground truth plays an important role in the classification process. The classifier uses part of the data for which the corresponding class is known as training data in order to establish the discriminating criteria. The part of the ground truth which was not used for training purposes will afterwards be used for the assessment of the classification accuracy. The remaining data with unknown class could then be assigned to a class.

The classification accuracy refers to the correspondence between the class label assigned to each sample and the "true" class obtain by the ground truth. Fuzzy class boundaries and incorrectly assigned classes are two main degrading factors of classification accuracy. Additionally, if the ground truth does not represent all classes adequately the classification result and the corresponding accuracy may be unpredictable. 


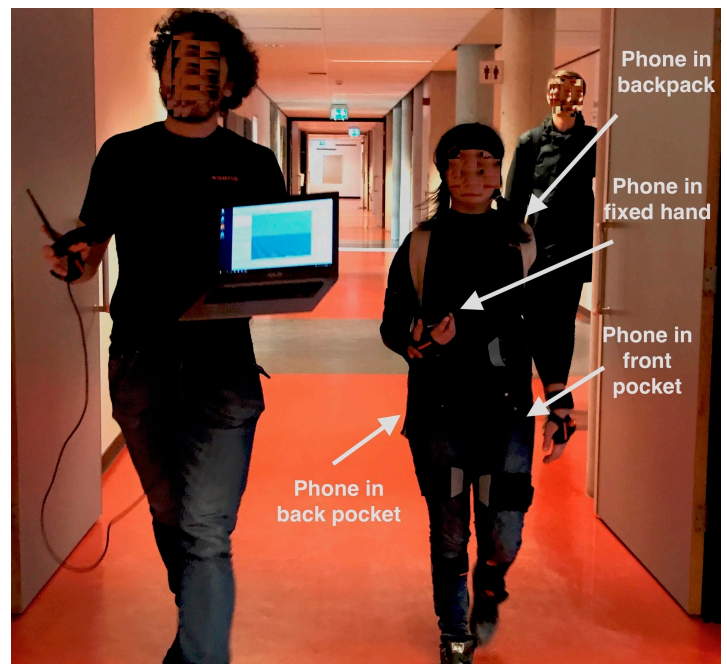

(a) The subject is walking constantly with almost constant speed and carrying a smartphone in flat and fixed hand.

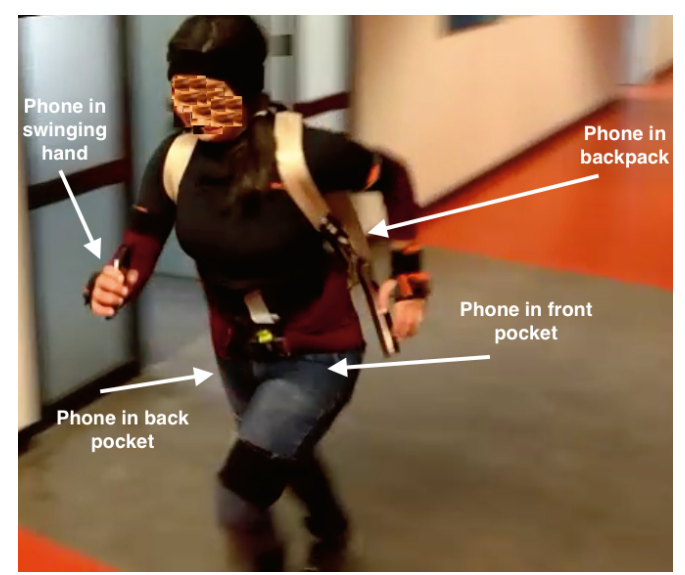

(b) The subject is running and carrying phone in swinging hand.

Figure 5.4: Photo from measurement campaign [49, C2017 IEEE].

As Figure 5.2 shows, MVN studio provides a movie of all the subject activities. We visually inspect these movies to determine the time of switch between the modes. However, the provided output of the MVN studio is a downsampled version of the signal. Thus, the visually obtained times need to be matched by the samples of the signal. We use the extracted output of the MVN studio that gives the exact time of each sample with milisecond(s) accuracy. All the samples are then labeled to appropriate classes using the derived time stamps used to form 
Table 5.4: Dataset details given from both smartphone and MTws. Signals from MTws are available in two versions; "Raw" data and filtered by some Biomechanical algorithms (BM). Smartphone data is only available in "Raw" version [49].

\begin{tabular}{|c|c|c|c|}
\hline Variable Names & Unit & MTw data & Phone data \\
\hline 3D AccR (Raw) & {$\left[\mathrm{m} / \mathrm{s}^{2}\right]$} & $\times$ & $\times$ \\
\hline 3D AngVelR(Raw) & {$[\mathrm{rad} / \mathrm{s}]$} & $\times$ & $\times$ \\
\hline 3D OriR (Raw) & {$\left[{ }^{\circ}\right]$} & $\times$ & $\times$ \\
\hline 3D MagR (Raw) & {$[\mathrm{Gauss}]$} & $\times$ & $\times$ \\
\hline GPS & {$[\mathrm{m}]$} & - & $\times$ \\
\hline Pressure & {$[\mathrm{Pa}]$} & - & $\times$ \\
\hline Mtw Position & {$[\mathrm{cm}]$} & $\times$ & - \\
\hline Velocity & {$[\mathrm{m} / \mathrm{s}]$} & $\times$ & - \\
\hline 3-D Acc (BM) & {$\left[\mathrm{m} / \mathrm{s}^{2}\right]$} & $\times$ & - \\
\hline 3-D AngVel(BM) & {$[\mathrm{rad} / \mathrm{s}]$} & $\times$ & - \\
\hline 3-D Ang.Acc (BM) & {$\left[\mathrm{rad} / \mathrm{s}^{2}\right]$} & $\times$ & - \\
\hline Ori (BM) & {$\left[{ }^{\circ}\right]$} & $\times$ & - \\
\hline Joint Angle & {$\left[{ }^{\circ}\right]$} & $\times$ & - \\
\hline Ground Truth $(\mathbf{G T})$ & - & $\times$ & $\times$ \\
\hline
\end{tabular}

the ground truth.

The ground truth is separately formed for high and low quality devices using the labels obtained. Depending on the device mode, signals obtained from appropriate MTws should be considered. For example, if the goal is to investigate the scenario in which the smartphone is being carried in hand, the hand-mounted sensor is the one that mimics that behaviors best. Another example is the case where the phone is in pocket where the most appropriate MTw revealing same properties is the one mounted on the upper leg. Similarly, we further assumed the MTw on the pelvis to simulate the scenario where the phone is being carried in backpack.

\subsubsection{Acquire Data}

The dataset containing the scenarios is defined in Table 5.3 and is available from [1]. The logged data from the phones and the MVN studio are extracted with 100 and $60 \mathrm{~Hz}$ sampling frequencies, respectively. Data from both devices are merged into a MATLAB '.mat'-file. The naming of experiments is in line with the structure given in Table 5.3. This file contains the structure of the data and attributes corresponding to each subject. For example, all measurements related to Case 4, are stored in Case 4 dataset and Case 4 . Subject (1) provides the data for all sensors and attributes associated with subject 1 .

Table 5.4 represents all available data for both devices in the measurement scenarios dataset. During the post-analysis phase of the data, some signals were identified as either corrupted or missing. As a result, the dataset contains some 
empty fields. The dataset contains 4 measurement scenarios with 7-8 measurement sets each, depending on the number of participants. To further simplify working with the dataset, a toy example with MATLAB code to extract the data is provided together with the dataset. The first measurement scenario (Case 1) for first subject is set as default. More details about setting variables and extracting desired outputs is provided in a README file attached to the dataset.

\subsection{Data Analysis}

We apply a classification algorithm to the data to assign them to the defined classes in Table 5.1. This is done in two steps; feature extraction on the raw signals, followed by a classification step.

\subsubsection{Feature Extraction}

Feature extraction is a way to bring out the inherent information in the available data, and reduce the dimensionality of the raw/pre-processed data in order to be able to apply classification algorithms on it.

The feature extraction phase is performed by dividing the inertial data in sliding windows of $N$ samples with no overlap. The window size must be selected such that it satisfies two different objectives. On one hand, it must be long enough to cover at least one gait cycle. On the other hand, it should be short enough to identify sudden motion mode transitions. In this work, we set the window size to 0.5 seconds. This translates to 50 and 30 samples for phone and motion trackers, respectively.

In the rest of this section we define the features we feed to the classifier and provide more specific examples from the dataset. All given examples correspond to Case 1 described in Table 5.3 where the subject intentionally switches between the fixed and swinging device modes.

\section{Signal Norm}

For a generic signal $S[n],\|S\|_{\max }$ denotes the maximum norm over the sampling window as follows

$$
\|S\|_{\max }=\max _{n}\|S[n]\| .
$$

The norm contains useful information used to discriminate between different modes. More precisely, any change of motion mode results in a large difference in the values of accelerometer norm, $\|a\|_{\max }$. This enables the identification of a change in the motion mode. In addition, a large difference in the values of the gyroscope norm $\|\omega\|_{\max }$ can be translated into a switch in the device mode.

To further illustrate the behavior of $\|a\|_{\max }$ relative to the change in the motion mode see Figure 5.5, where the norms of signals are depicted while the smartphone is in both fixed and swinging modes. These signals correspond to Case 1 in Figure 5.3. Figure 5.5b further presents how the gyroscope norm, $\|\omega\|_{\max }$, assists in device mode discrimination. 


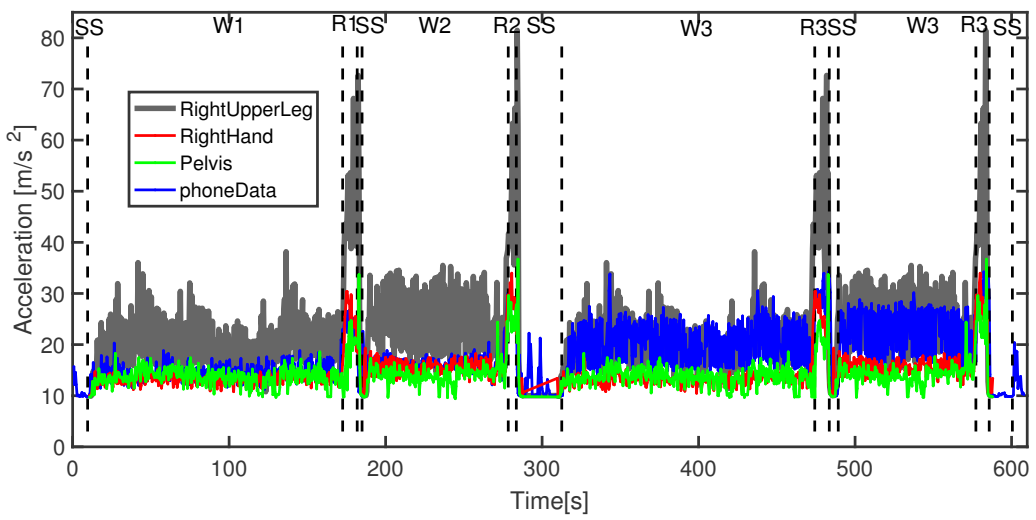

(a) Norm of accelerometer signal.

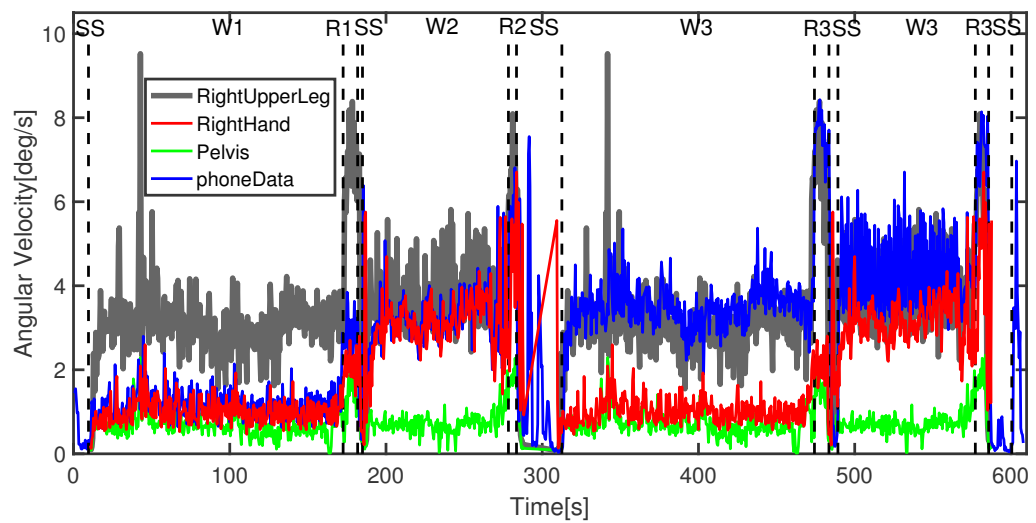

(b) Norm of gyroscope signal.

Figure 5.5: Signal norm for 4 different motion trackers together with the signal from the smartphones. The subject performed the Case 1 from Figure 5.3. Black lines separate the different classes. Related class for each section is indicated in the figures. The subject carried a smartphone in the right hand and one in the front right pocket. The device carried by hand is switching between fixed and swinging mode [49, (C2017 IEEE].

\section{Signal Energy}

The energy of the signal, $E_{S}$, is obtained by taking the squared norm of $S[n]$ and summing

$$
E_{S}=\sum_{n=0}^{N-1}\|S[n]\|^{2} .
$$




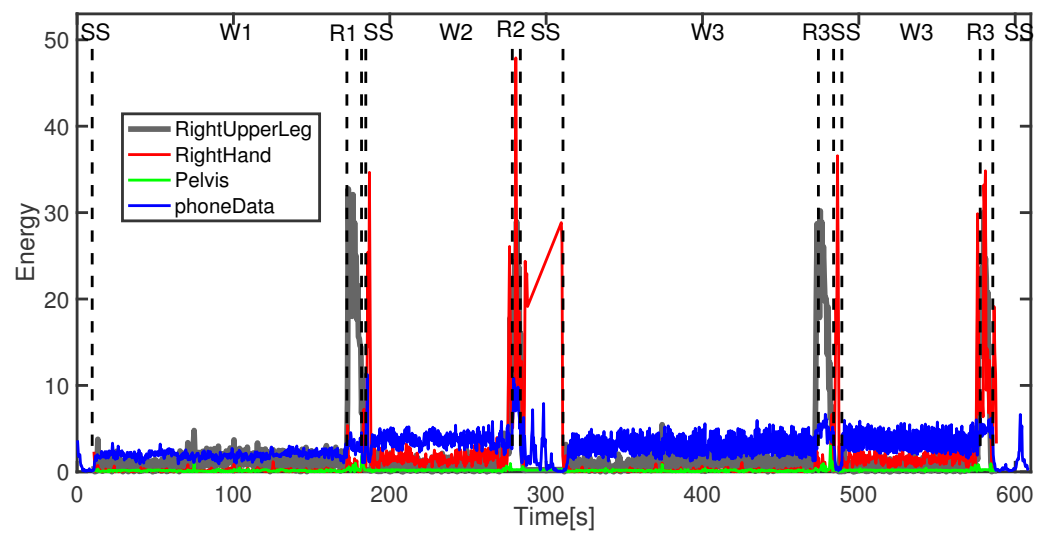

(a) Accelerometer energy signal.

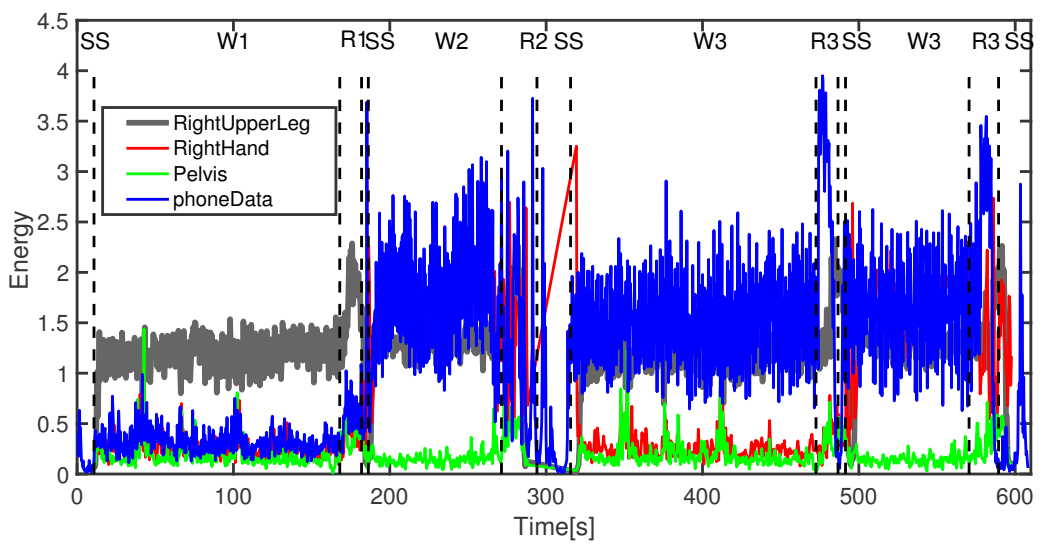

(b) Gyroscope energy signal.

Figure 5.6: Energy signal for 4 different motion trackers together with the signal from the smartphones. The sensor locations and measurement scenario are the same as the one described in Figure 5.5 [49, (C2017 IEEE].

The obtained energy is a useful feature allowing us to distinguish fixed or moving device modes due to rapid response to mode transition. For example, the gyroscope will have higher energies in swinging hand device mode than the fixed hand scenario.

Figure 5.6 shows the energy signal for both accelerometer and gyroscope. As shown in Figure 5.6a each transition between motion modes results in a noticeable change in the energy of the accelerometer signal. The effect of the device mode on the energy signal is illustrated in Figure 5.6b, where it is shown that switching from fixed to swinging mode increases the angular velocity energy drastically. 


\section{Signal variance}

For any generic signal $S[n]$ the average of the squared differences from the mean, the variance signal, is defined as follows

$$
\sigma_{S}^{2}=\frac{1}{N-1} \sum_{n=0}^{N-1}\left(\|S[n]\|-\frac{1}{N} \sum_{n=0}^{N-1}\|S[n]\|\right)^{2} .
$$

The variance signal assists to discriminate between high and low intensity movements. For example, the estimated variance of both accelerometer and gyroscope is highly informative while the objective is to distinguish between swinging mode with any other less intense movement scenario.

Figure 5.7 shows the signal variance of both accelerometer and gyroscope. One advantage of the variance signal is to enhance robustness of the classification algorithm in motion mode recognition. Running leads to more intense movements than both walking and standing still. Higher peaks in the signal variance, as shown in Figure 5.7a, can then be identified once the user switches to this mode. Additionally, the signal variance analysis can be used to recognize device mode switches. The variation of the variance signal illustrated in Figure 5.7b shows how changing between classes with different device modes, W1 and W2 for example, can be distinguished.

\section{Frequency Analysis}

This feature allows us to identify any movement such as periodic movement from aperiodic ones. Different activities have different frequencies, resulting in a varying power spectrum that assists in activity identification. Thus, the analysis of the frequency domain of inertial signals recorded with hand-held devices allows capturing the periodicity of the accelerometer/gyroscope signals due to the subject's activity. Presence or absence of peaks in the spectogram of the inertial signals gives useful insights whether the subject is having a periodic movement or standing still.

The spectogram of the gyroscope signal is obtained using Short Time Fourier Transform (STFT) and is reported in Figure 5.8. The periodicity of the walking and running mode is visible in the frequency peaks of the spectogram while it is possible to identify a static case around time 300.

\subsubsection{Classification}

As a final step in characterizing the data, a classifier is applied to the features to try to extract the motion and device modes as defined in Table 5.1.

Different machine learning algorithms can be used for the classification. For example, [90] applies a decision tree for classification purposes while [102] uses MLP and a SVM to improve the performance in terms of recognition of human activity.

Two datasets, are formed for phone and motion tracker signals as described in Section 5.2.2, separately. Each set contains eight features constructed from four 


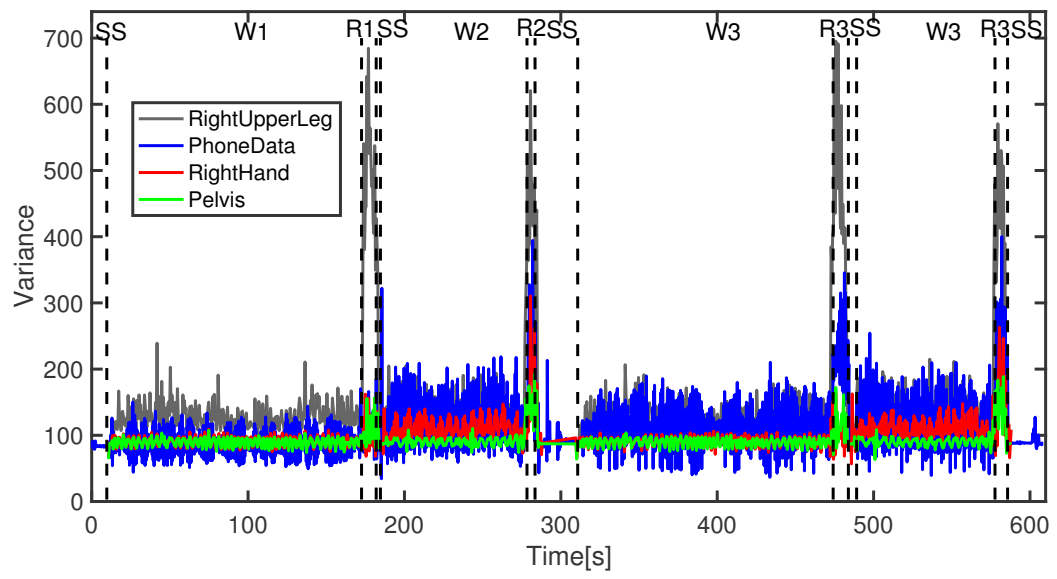

(a) Accelerometer signal variance.

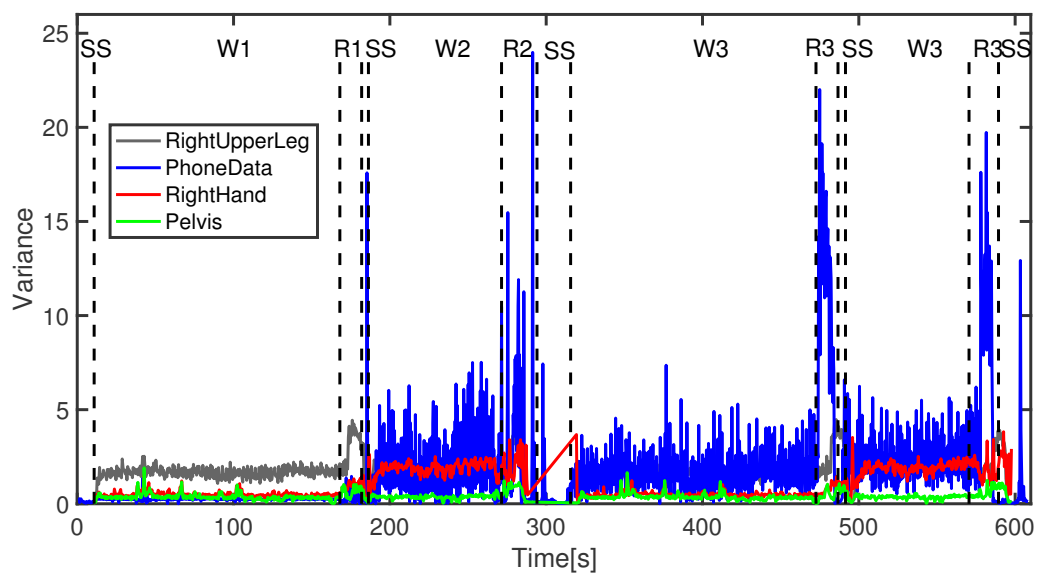

(b) Gyroscope signal variance.

Figure 5.7: Signal variance for 4 different motion trackers together with the signal from the smartphones. The sensor locations and measurement scenario are the same as the one described in Figure 5.5 [49, (C2017 IEEE].

signal attributes introduced in Section 5.3 for both gyroscope and accelerometer. Since the classes are labeled accurately for each dataset, a supervised learning approach is applied. A multivariate decision tree classifier is trained using MATLAB; Machine learning toolbox. The classifier is validated with 10 -fold crossvalidation.

The performance of the classifier for both devices are summarized in the confusion matrices given in Tables 5.6a and 5.6b. In the MTw data, having a sensor 


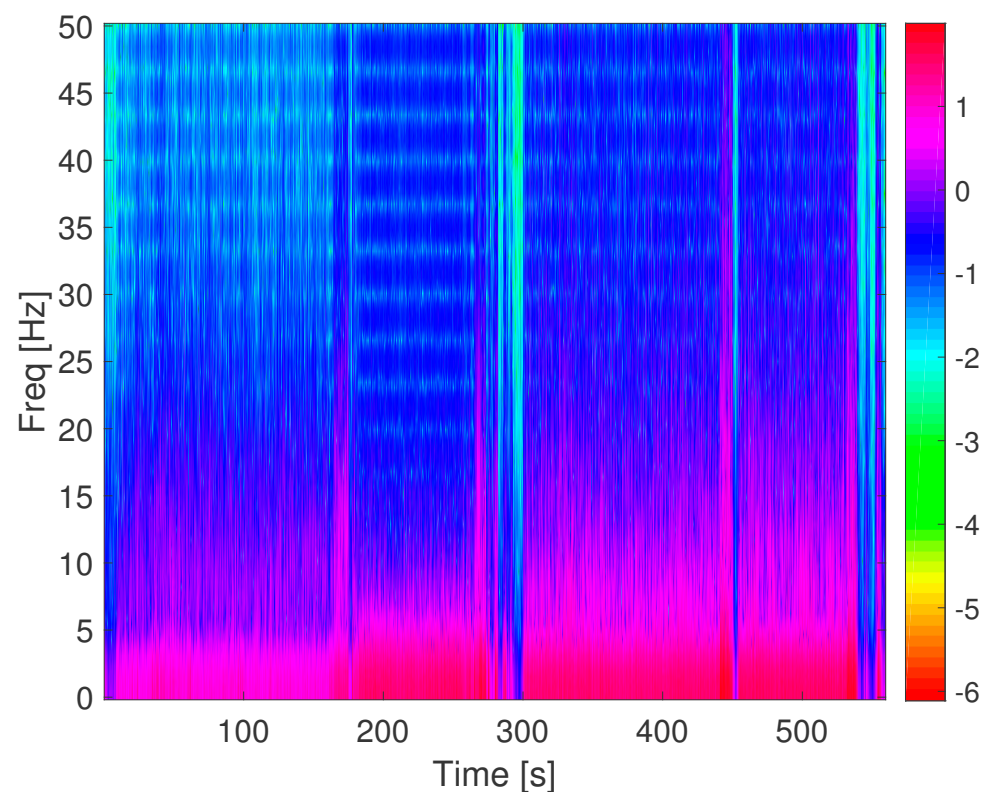

Figure 5.8: Spectogram of the smartphone's gyroscope signal carried in the right hand. The subject performed the Case 1 from Figure 5.3 [49, C2017 IEEE].

to mimic the behavior of the phone data carried by backpack is challenging. As Table 5.6a reports, the mode detection success rate for MTw sensors are over 70\% for all classes. SS and W1 classes are missclassified with W4 class corresponding to the pelvis sensor. To explain this observation, one needs to note how the pelvis sensor is rigidly mounted on the body. This leads to miss-classification with other low intensity modes. With phone data, the classification results are promising as represented by Table 5.6b. All the classes are detected correctly with over $75 \%$ for walking and standing still modes and over $56 \%$ for running mode. Running mode recognition is challenging as the phone has movement. Missclassification of R1 with R4 and W1 with W4 can be explained by the fact that the phone is fixed in either of these situations. 
Table 5.5: Confusion matrix of the modes classification represented in Table 5.1 using the binary decision tree classifier using cross-validation with 10 folds. The table shows how different annotated activities are classified in [\%].

\begin{tabular}{|c|c|c|c|c|c|c|c|c|c|}
\hline & \multicolumn{9}{|c|}{ Recognised activity } \\
\hline & \& & $p^{2}$ & $R^{3}$ & $e^{*}$ & $s^{5}$ & $\$$ & $\Delta^{2}$ & $N^{3}$ & $s^{x}$ \\
\hline R1 & 83 & 8 & 0 & 6 & 0 & 0 & 0 & 0 & 0 \\
\hline $\mathrm{R} 2$ & 5 & 72 & 1 & 0 & 0 & 0 & 0 & 1 & 0 \\
\hline R3 & 0 & 2 & 96 & 0 & 0 & 0 & 0 & 0 & 0 \\
\hline $\mathrm{R} 4$ & 5 & 0 & 0 & 85 & 1 & 0 & 0 & 0 & 1 \\
\hline SS & 0 & 2 & 0 & 1 & 79 & 2 & 0 & 0 & 1 \\
\hline W1 & 0 & 0 & 1 & 1 & 5 & 78 & 1 & 0 & 13 \\
\hline W2 & 0 & 8 & 0 & 0 & 0 & 1 & 84 & 6 & 0 \\
\hline W3 & 7 & 8 & 1 & 3 & 2 & 1 & 13 & 92 & 0 \\
\hline W4 & 0 & 0 & 1 & 4 & 13 & 17 & 1 & 0 & 84 \\
\hline
\end{tabular}

(a) Data from 4 different motion trackers

\begin{tabular}{|c|c|c|c|c|c|c|c|c|c|}
\hline & \multicolumn{9}{|c|}{ Recognised activity } \\
\hline & \& & $\Omega^{2}$ & $\beta^{3}$ & $e^{*}$ & ss & $\$$ & $\mathbf{s}^{2}$ & $N^{3}$ & $a^{x}$ \\
\hline R1 & 58 & 3 & 0 & 0 & 0 & 0 & 0 & 2 & 0 \\
\hline R2 & 1 & 60 & 4 & 4 & 0 & 0 & 1 & 2 & 0 \\
\hline R3 & 0 & 13 & 83 & 0 & 1 & 0 & 0 & 0 & 0 \\
\hline R4 & 20 & 3 & 0 & 56 & 0 & 0 & 0 & 2 & 0 \\
\hline SS & 1 & 0 & 6 & 0 & 84 & 4 & 3 & 2 & 1 \\
\hline W1 & 3 & 0 & 0 & 7 & 7 & 83 & 3 & 1 & 8 \\
\hline W2 & 1 & 3 & 6 & 7 & 2 & 1 & 77 & 14 & 0 \\
\hline W3 & 14 & 17 & 2 & 22 & 1 & 1 & 16 & 75 & 0 \\
\hline W4 & 0 & 0 & 0 & 4 & 5 & 11 & 1 & 0 & 90 \\
\hline
\end{tabular}

(b) Data from phones. 


\subsection{Conclusion}

This work presents an extensive dataset for classification and investigation of the motion and device mode for PNS, where many device modes, a number of users and a few motion modes are logged for three different trajectories. All data are fully annotated with ground truth classes, and other metadata such as position and accurate full body motion using biomeachnical models. We have discussed how the motion and device mode relate to pedestrian dead-reckoning algorithms, the working horse of PNS, and how PNS can otherwise benefit from improved and extended mode classification. The larger part of the paper described the field tests in detail. The last part suggests a few features that can be computed from the logged data, and applies a straight forward classifier on these features. The result is quite promising, yet preliminary. The dataset is publicly available [1], and we hope the research community can benefit from this to improve the classification results further. 



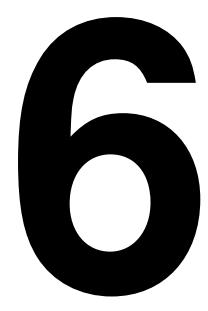

\section{Asynchronous Averaging of Gait Cycles}

This work is submitted to:

P. Kasebzadeh, G. Hendeby, and F. Gustafsson. Asynchronous averaging of gait cycles for classification of gait and device modes. Submitted to IEEE Submitted to IEEE Sensors Journal, 2019a, and available in: eprint arXiv: arXiv:1907.02329v2.

An approach for computing unique gait signature using measurements collected from body-worn IMUs is proposed. The gait signature represents one full cycle of the human gait, and is suitable for off-line or on-line classification of the gait mode. The signature can also be used to jointly classify the gait mode and the device mode. The device mode identifies how the IMU-equipped device is being carried by the user. The method is based on precise segmentation and resampling of the measured IMU signal, as an initial step, further tuned by minimizing the variability of the obtained signature within each gait cycle. Finally, a Fourier series expansion of the gait signature is introduced which provides a low-dimensional feature vector well suited for classification purposes.

The proposed method is evaluated on a large dataset involving several subjects, each one containing two different gait modes and four different device modes. The gait signatures enable a high classification rate for each step cycle.

The remainder of this chapter is organized as follows: Section 6.1 describes the considered problem. In Section 6.2, a standard threshold-based method for step detection is given followed by the solution to the gait cycle optimal segmentation problem. Section 6.3 presents a Fourier series approach to achieve a lowdimensional feature vector. The performance of the proposed method is evaluated in Section 6.4, which also includes an optional data pre-processing stage followed by the concluding remarks given in Section 6.5. 


\subsection{Problem Formulation and Notation}

Consider some physical quantity $\boldsymbol{x}(s)$, possibly multi-dimensional, that depends on gait, which can be measured with additive noise $\boldsymbol{e}(s)$ as

$$
y\left(s_{n}\right)=\boldsymbol{x}\left(s_{n}\right)+\boldsymbol{e}\left(s_{n}\right), \quad n=1,2, \ldots, N,
$$

where $s_{n}$ denotes the sampling times. We assume that the gait cycle is periodic over periods of time, and we are interested in the underlying average gait cycle of the physical quantity $g(\tau)$ on a normalized time scale $0 \leq \tau<1$. Figure $6.1 \mathrm{a}$ is an example to represent periodic gait cycles over the normalized time scale. The presented data in Figure 6.1a corresponds to a real scenario in which the walking subject was carrying a smartphone in the hand, facing upwards. All the gait segments in the figure were extracted manually from a bandpass-filtered accelerometer signal.

The challenge is to estimate the gait cycle $\hat{\boldsymbol{g}}(\tau)$ from the IMU measurements $y(s)$, and the most critical step is the segmentation in which the signal is split into the separate gait cycles. We denote the beginning of each gait cycle time by $t_{m}$ such that

$$
s_{1} \leq t_{0}<t_{1}<\cdots<t_{M} \leq s_{N} .
$$

Figure $6.1 \mathrm{~b}$ shows a histogram of step time duration, $t_{m}-t_{m-1}$, for all $M$ gait cycles in Figure 6.1a. Given these durations, we can obtain a estimate of the gait cycle, Figure 6.1a, from each segment as

$$
\hat{\boldsymbol{g}}_{m}(\tau)=x\left(t_{m-1}+\left(t_{m}-t_{m-1}\right) \tau\right), \quad \tau \in[0,1) .
$$

Then, we immediately get what is referred to as an asynchronously averaged gait cycle by

$$
\overline{\boldsymbol{g}}(\tau)=\frac{1}{M} \sum_{m=1}^{M} \hat{\boldsymbol{g}}_{m}(\tau) .
$$

The green line in Figure 6.1a represents $\overline{\boldsymbol{g}}(\tau)$ for all manually extracted gait cycles.

The key problem is thus to determine the step times such that different gait cycles $\hat{\boldsymbol{g}}_{m}(\tau)$ become as similar as possible. The considered metric as a measure of the similarity is the variance between each gait cycle and the averaged gait signature. We propose a nonlinear least squares framework where we optimize the step times in order to minimize the variance of these gait cycles

$$
\begin{aligned}
\hat{t}_{0: M} & =\arg \min _{t_{0: M}} V\left(t_{0}: t_{M}\right), \\
V\left(t_{0}: t_{M}\right) & =\frac{1}{M} \sum_{m=1}^{M}\left\|\overline{\boldsymbol{g}}(\tau)-\hat{\boldsymbol{g}}_{m}(\tau)\right\|^{2} .
\end{aligned}
$$

To make the optimization problem mathematically tractable, we will approximate the optimization problem in the following ways: 


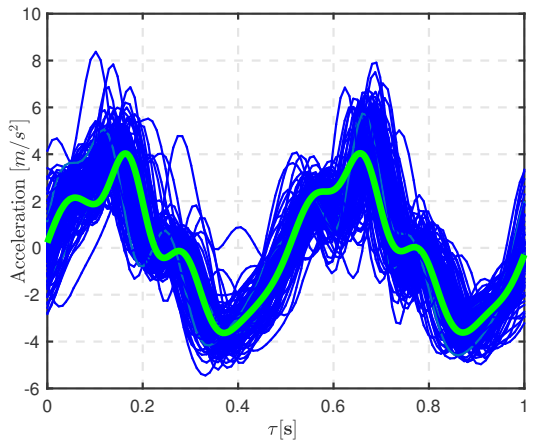

(a) Gait cycles, marked with blue lines, and averaged gait signature, marked with the green line.

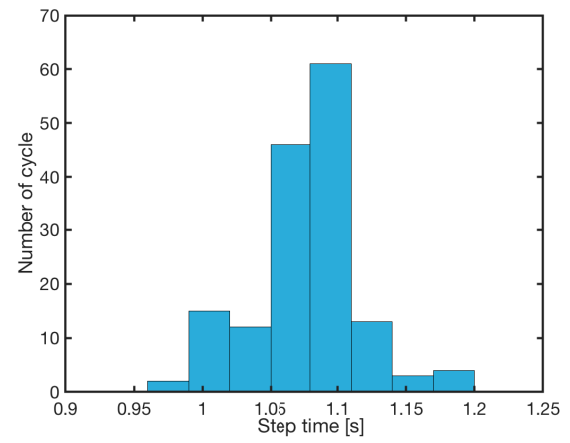

(b) Histogram of step times.

Figure 6.1: Gait cycles, manually segmented using accelerometer signal.

1. The normalized time scale is discretized to $L$ uniformly spaced grid points $\tau_{l}=(l-1) / L$ for $l=1,2, \ldots, L$.

2. The $L_{2}$-norm is used in $(6.5 \mathrm{~b})$, which gives a nonlinear least squares (NLS) problem, which often allows for efficient solvers.

Based on these assumptions, the problem can be written as

$$
\hat{t}_{0: M}=\arg \min _{t_{0: M}} \frac{1}{L M} \sum_{m=0}^{M} \sum_{l=1}^{L}\left(\overline{\boldsymbol{g}}\left(\tau_{l}\right)-\hat{\boldsymbol{g}}_{m}\left(\tau_{l}\right)\right)^{2} .
$$

These two restrictions also enable us to formulate the signal Fourier series (FS) expansion in order to reduce the model order as introduced in Section 6.3.

A solution for this optimization problem is proposed in Section 6.2.1. Moreover, we will use a rather standard step detection method based on thresholding, outlined in Section 6.2.2, in order to initialize the optimization problem.

\subsection{Optimal Segmentation of Gait Cycles}

In this section, we first suggest a solution to the optimization problem (6.6). Then, a standard threshold-based step detection method is presented in order to initialize the optimization algorithm.

It is worth noting that all solutions are provided based on the assumption that the measurements are collected in advance (the solution is for the off-line mode). However, it could be easily extended to the on-line problems. Moreover, during the optimization process, all the gait cycles should be in the same regime of gait and device modes introduced in Table 5.1. 


\subsubsection{Solution for Optimization Problem}

The sensor data should be band-pass filtered before segmentation to remove slow trends and high frequency noise. The cut-off limits in the band-pass filter should be selected to take the slowest and fastest pace into account, see Section 6.2.2 for further details. Moreover, the minimum step time $\epsilon_{l o}$ and the maximum step time $\epsilon_{u p}$ are required to be defined in advance. These bounds can be obtained from the most frequent time interval of the gait cycles given by the histogram, e.g. Figure $6.1 \mathrm{~b}$, of the detected gait cycles. The estimated step cycles should be in this interval.

The minimization problem for the objective function $V\left(t_{0}: t_{M}\right)$ is given by

$$
\begin{array}{cl}
\underset{t_{0}: t_{M}}{\operatorname{minimize}} & V\left(t_{0}: t_{M}\right) \\
\text { subject to } & \epsilon_{l o}<t_{m}-t_{m-1}<\epsilon_{u p}
\end{array}
$$

In order to make the optimization procedure feasible, it is reformulated into $M$ sub-optimal problems where each problem requires the optimal solution from the previous one. The first sub-problem is defined by

$$
\begin{array}{ll}
\underset{t_{1}}{\operatorname{minimize}} & V\left(t_{0}: t_{M}\right), \\
\text { subject to } & \epsilon_{l o}<t_{1}-t_{0}<\epsilon_{u p}
\end{array}
$$

where the outcome of the problem would be the optimal value of $t_{1}$ denoted by $\hat{t}_{1}$. Given $\hat{t}_{1}$, the second sub-problem becomes

$$
\begin{array}{ll}
\underset{t_{2}}{\operatorname{minimize}} & V\left(t_{0}, \hat{t}_{1}, t_{2}: t_{M}\right), \\
\text { subject to } & \epsilon_{l o}<t_{2}-\hat{t}_{1}<\epsilon_{u p}
\end{array}
$$

The optimized $\hat{t}_{2}$ is the outcome of the second step.

To generalize, the $i:$ th $i \in[2, \ldots, M]$ sub-problem is given by

$$
\begin{array}{ll}
\underset{t_{i}}{\operatorname{minimize}} & V\left(t_{0}, \hat{t}_{1}: \hat{t}_{i-1}, t_{i}: t_{M}\right) . \\
\text { subject to } & \epsilon_{l o}<t_{i}-\hat{t}_{i-1}<\epsilon_{u p}
\end{array}
$$

These simplified optimization problems could be solved using a general linear search method algorithm which iteratively minimizes the cost function considering the given boundaries. Derivative-free quadratic interpolation and golden section search methods are used in this work [66].

Algorithm 3 outlines the proposed solution to find the optimal gait cycle segments from a given accelerometer signal. To initialize the algorithm, the preliminary gait cycles, $\hat{\boldsymbol{g}}_{1: M}(\tau)$, are detected using a classical threshold-based step detection algorithm as described in Section 6.2.2. The initial signature, $\overline{\boldsymbol{g}}(\tau)$, is then estimated by (6.4) considering all detected $\hat{\boldsymbol{g}}_{1: M}(\tau)$. Subsequently, the cost function, $V\left(t_{0}: t_{M}\right)$, introduced in $(6.5 \mathrm{~b})$ is computed using $\overline{\boldsymbol{g}}(\tau)$ and $\hat{\boldsymbol{g}}_{1: M}(\tau)$. For each gait cycle, $\hat{\boldsymbol{g}}_{i}(\tau)$, the algorithm strives to find the optimal value $\hat{t}_{i}$ using $(6.10)$. Additionally, the initial gait signature is updated based on the new set of $t$. 


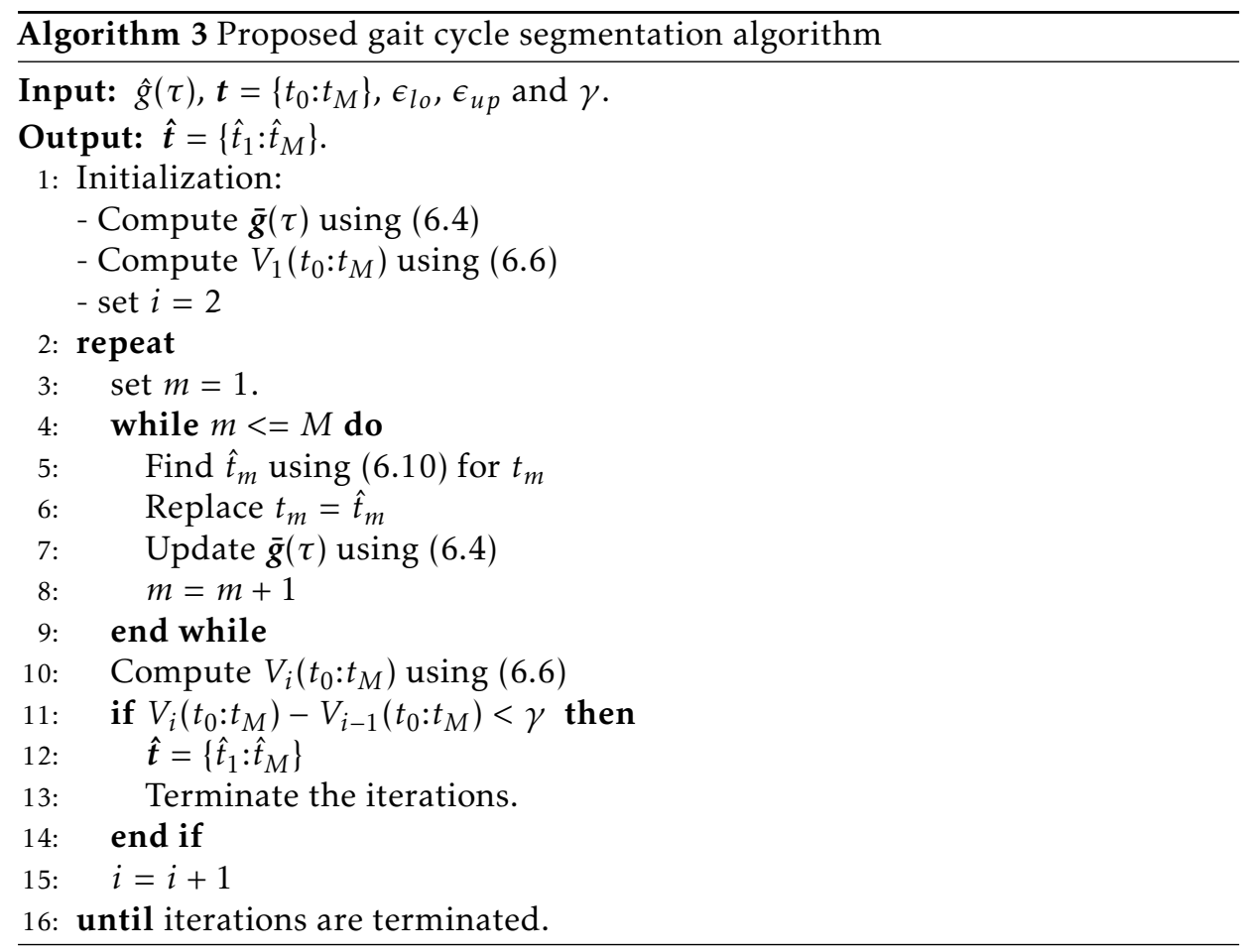

Once all the detected gait cycles have been optimized, the cost function, $V\left(t_{0}: t_{M}\right)$, will be re-computed given the estimated gait cycles. Henceforth, the new optimal gait cycles and updated signature will be used as the initialization for the next iteration of optimization problems. The termination criterion for this iterative algorithm is based on the decrement on the cost function. Hence, the algorithm will be iterated until the absolute decrease of cost function falls below the given threshold $\gamma$.

The performance of the optimization problem is evaluated for all introduced scenarios in Table 5.1 in Section 6.4.

\subsubsection{Classical Gait Segmentation}

To initialize the algorithm for offline applications, a threshold based step detection method is employed. A gait cycle, containing two consecutive toe-off moments of the same foot, can be crudely detected by defining two thresholds. If the peak threshold $\epsilon_{p}$ and the valley threshold $\epsilon_{v}$ are each crossed twice, then a cycle is taken. The measured signal will be compared against both $\epsilon_{p}$ and $\epsilon_{v}$ to find the time interval that it takes for the signal to cross both thresholds twice. Each gait cycle is then defined as sample values along this interval.

All the steps of the threshold-based step detection algorithm are summarized in Algorithm. 4. As a first step, in the gait cycle detection algorithm, we need to define the hyperparameter values for $\epsilon_{p}$ and $\epsilon_{v}$. These values depend on human 
activity mode and device mode. Different approaches are introduced to consider proper thresholds, either pre-defined or adaptive [48, 57, 102]. In this work, predefined thresholds on norm of acceleration value have been used to detect peaks and valleys.

By considering a proper threshold for each scenario, the gait cycle can be extracted by comparing the norm of the acceleration signal at each time with the $\epsilon_{p}$ or $\epsilon_{v}$. Peak and valley should be hit sequentially. However, we do not know which one comes first. Hence, in order to make sure that both peaks and both valleys have been detected in order, two flags $h i t_{p}$ or $h i t_{v}$ are defined.

Algorithm 4 is applied to the experimental dataset (containing 8 scenarios), as introduced in Section 6.4 to get the gait cycle segmentations. The hyperparameter values are set to $\epsilon_{p}=2 \mathrm{~m} / \mathrm{s}^{2}$ and $\epsilon_{v}=-2 \mathrm{~m} / \mathrm{s}^{2}$ for walking mode and $\epsilon_{p}=4 \mathrm{~m} / \mathrm{s}^{2}$ and $\epsilon_{v}=-5 \mathrm{~m} / \mathrm{s}^{2}$ for running mode. The extracted segments are illustrated in Figure 6.2. It is worth noting that all the gait cycle times are normalized such that $\tau \in[0,1)$.

In this work, in order to extract the pattern of the gait cycle with better quality and to avoid bias drift, a pre-processing step is applied to the raw measured data. For this purpose, the signal is filtered through a fourth-order Butterworth filter.The norm of the filtered signal is considered in this work in order to avoid unpredicted disturbances of the vertical acceleration that the orientation of the sensor may cause.

The initial gait signatures, $\mathbf{\mathbf { g }}(\tau)$, computed by (6.4) are plotted over the detected gait cycles for all the scenarios and indicated with thick green line in Figure 6.2. As the figures suggest, although a general pattern is visible for the extracted gait cycles in all scenarios, further tuning is required. For example, when the device is in swinging mode, Figure $6.2 \mathrm{c}$ and Figure $6.2 \mathrm{~d}$, corresponding to walking and running with swinging hand, the gait cycles are very noisy and there are some misdetected gait segments. Moreover, in "W4" scenario, presented in Figure $6.2 \mathrm{~g}$, corresponding to walking with backpack device mode, there is some shifting. In the running activity mode the patterns are quite noisy and the lengths of the cycle times also vary as shown in the histograms corresponding to running gait modes.

Unexpected behaviors in the motion and the device modes are unpredictable and always exist in pedestrians' daily activities. Hence, these classical algorithms such as the one given by Algorithm 4 should be complemented by more advanced methods.

The introduced optimal segmentation in Section 6.2.1, suggests a solution to take care of unexpected behaviors and reduce the rate of error. The performance of the proposed method is evaluated on a large dataset and presented in Section 6.4.

\subsection{Data Reduction with Fourier Series}

In this section, we are looking for a low order approximation, which can be recasted as a least squares (LS) estimation problem using a linear regression frame- 
Algorithm 4 Gait cycle detection

Input: Norm of accelerometer signal

$$
\|\boldsymbol{a}(k)\|=\sqrt{\boldsymbol{a}_{x}^{2}+\boldsymbol{a}_{y}^{2}+\boldsymbol{a}_{z}^{2}}
$$

- Lower bound $\epsilon_{\text {lo }}$ and upper bound $\epsilon_{\text {up }}$ thresholds, if hit step is occurred Output: Set of gait cycles $\left\{Y_{m}\right\}_{m=1}^{M}$

1: Initialization:

- Set counters $c_{\text {up }}=0$ and $c_{\mathrm{lo}}=0$ representing the number of times upper and lower bounds are hit

- set hit $_{p}=$ FALSE and hit $_{v}=$ FALSE

$-k_{\text {start }}=1$

- $k=k_{\text {start }}$

$-m=1$

2: repeat

3: $\quad$ if $\|\boldsymbol{a}(k)\| \geq \epsilon_{\text {up }}$ then

4: $\quad c_{\text {up }}=c_{\text {up }}+1$

5: $\quad$ hit $_{p}=$ TRUE, hit $_{v}=$ FALSE

6: $\quad$ end if

7: $\quad$ if $\|\boldsymbol{a}(k)\| \leq \epsilon_{\text {lo }}$ then

8: $\quad c_{\mathrm{lo}}=c_{\mathrm{lo}}+1$

9: $\quad$ hit $_{p}=$ FALSE, hit $_{v}=$ TRUE

10: $\quad$ end if

11: $\quad k=k+1$

12: until hit $_{p}=\mathrm{TRUE}$ or $h i t_{v}=\mathrm{TRUE}$

13: while $c_{\text {up }}<2$ and $c_{\text {lo }}<2$ and $k \leq N$ do

14: $\quad$ if $\|\boldsymbol{a}(k)\| \geq \epsilon_{\mathrm{up}}$ and $h i t_{p}=$ FALSE and $h i t_{v}=$ TRUE then

15: $\quad c_{\text {up }}=c_{\text {up }}+1$

16: $\quad$ hit $_{p}=$ TRUE, hit $_{v}=$ FALSE

17: end if

18: if $\|\boldsymbol{a}(k)\| \leq \epsilon_{\mathrm{lo}}$ and $h i t_{p}=$ TRUE and $h i t_{v}=$ FALSE then

19: $\quad c_{\mathrm{lo}}=c_{\mathrm{lo}}+1$

20: $\quad$ hit $_{p}=$ FALSE, hit $_{v}=$ TRUE

21: end if

22: $\quad k=k+1$

23: end while

24: $\left\{Y_{m}\right\}=\left\|\boldsymbol{a}\left(k_{\text {start }}: k-1\right)\right\|$

25: $\left\{t_{m}\right\}=t_{\text {acc }}(k-1)$

26: $k_{\text {start }}=k$

27: $m=m+1$

28: $c_{\text {up }}=0$ and $c_{\text {lo }}=0$

29: return $\{\boldsymbol{Y}\},\{\boldsymbol{t}\}$ 

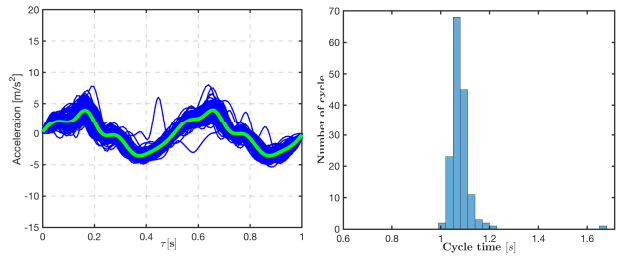

(a) $W 1$
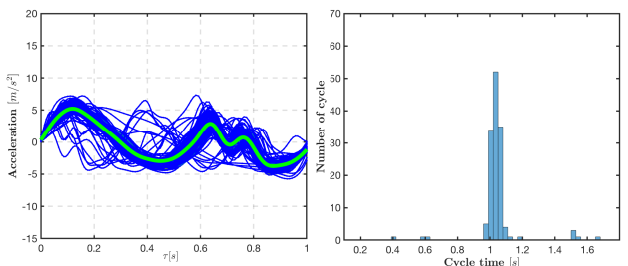

(c) $W 2$
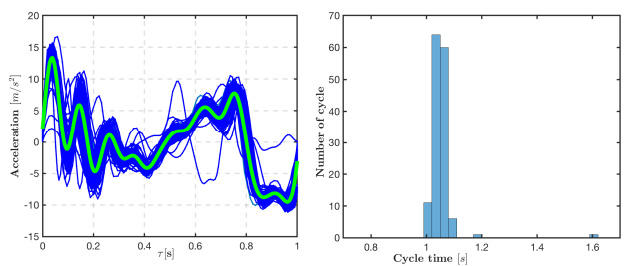

(e) $W 3$
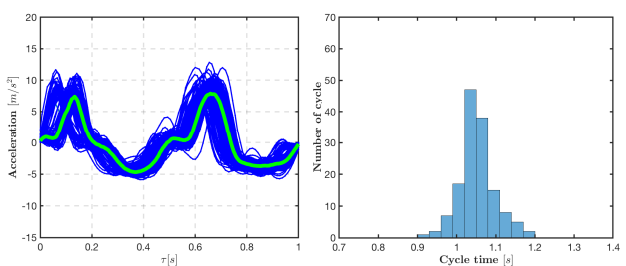

(g) $W 4$
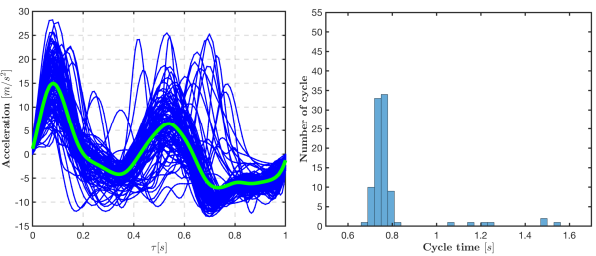

(b) R1
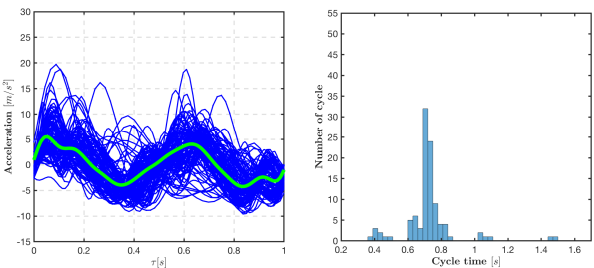

(d) $R 2$
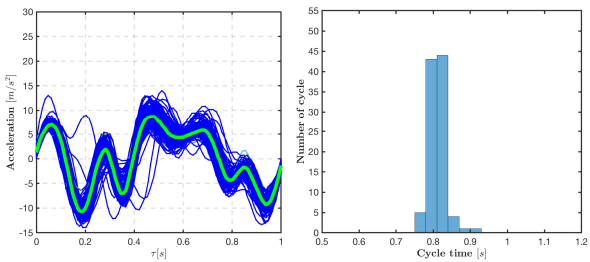

(f) $R 3$
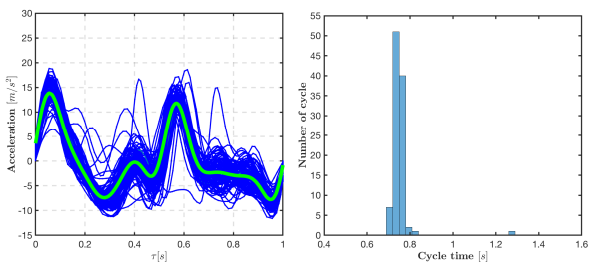

(h) $R 4$

Figure 6.2: Results for all eight introduced scenarios in Table 5.1. In each sub-figure: On the left, detected gait cycles (blue lines) using Algorithm 4 are presented and estimated reference signal $\overline{\boldsymbol{g}}(\tau)$ is depicted on top of all detected gait cycles with a solid thick green line. On the right, the histogram for the gait cycle duration time is presented. 
work.

Given our sequence of step times and the measurement noise we have for each $\hat{g}_{m}\left(\tau_{l}\right)$, we strive to find a parametric model, using a linear regression framework, of the average $\bar{g}\left(\tau_{l}\right)$. We show that the parameters of the fitted model provide a useful set of features for future classification purposes.

In order to extract a low dimensional feature vector for the gait cycle using estimated signatures, we apply FS expansions to the gait segment from the sampled version of the gait cycle $\hat{\boldsymbol{G}}_{m}[l], l=[0,1, \ldots, L-1]$

$$
\hat{\boldsymbol{G}}[l]=\sum_{k=0}^{K-1} a_{k} \cos \left(2 \pi \tau_{l} k\right)+b_{k} \sin \left(2 \pi \tau_{l} k\right),
$$

where the parameter set $\left\{a_{k}, b_{k}\right\}_{k=1}^{K}$ forms the feature space used to identify each particular gait mode and $K$ is the model order. As in any other regression model, the trade-off between model complexity and accuracy cannot be neglected. In order to estimate the FS coefficients, for each model order $K$, the Fourier series expansion (6.11) is considered as a linear model given by

$$
\bar{g}=H_{K} \theta_{K}
$$

where $\theta$ is a vector containing all unknown coefficients, where can be define as $\theta_{K}^{2 K \times 1}=\left[a_{0}, a_{1}, \ldots, a_{K-1}, b_{0}, b_{1}, \ldots, b_{K-1}\right]^{\top}$, and $\overline{\boldsymbol{g}}^{L \times 1}=\left[\overline{\boldsymbol{g}}\left(\tau_{0}\right), \overline{\boldsymbol{g}}\left(\tau_{1}\right), \ldots, \overline{\boldsymbol{g}}\left(\tau_{L-1}\right)\right]^{\top}$. $\boldsymbol{H}_{K}^{\top} \in \mathbb{R}^{2 K \times L}$ can then be given by

$$
\boldsymbol{H}_{K}^{\top}=\left[\begin{array}{cccc}
1 & \cos \left(2 \pi \tau_{1}\right) & \ldots & \cos \left(2 \pi \tau_{L-1}\right) \\
\vdots & \vdots & \ldots & \vdots \\
1 & \cos \left(2 \pi \tau_{1}(K-1)\right) & \ldots & \cos \left(2 \pi \tau_{L-1}(K-1)\right) \\
0 & \sin \left(2 \pi \tau_{1}\right) & \ldots & \sin \left(2 \pi \tau_{L-1}\right) \\
\vdots & \vdots & \ldots & \vdots \\
0 & \sin \left(2 \pi \tau_{1}(K-1)\right) & \ldots & \sin \left(2 \pi \tau_{L-1}(K-1)\right)
\end{array}\right] .
$$

The solution to the problem is obtained by finding the following optimization problem

$$
\hat{\theta}=\underset{\theta}{\operatorname{minimize}} V^{L S}(\boldsymbol{\theta}),
$$

where

$$
V^{L S}\left(\boldsymbol{\theta}_{K}\right)=\left(\overline{\boldsymbol{g}}-\boldsymbol{H}_{K} \boldsymbol{\theta}_{K}\right)^{\top}\left(\overline{\boldsymbol{g}}-\boldsymbol{H}_{K} \boldsymbol{\theta}_{K}\right) .
$$

Finally, the closed form solution $\hat{\theta}$ is given by

$$
\hat{\boldsymbol{\theta}}_{K}=\left(\boldsymbol{H}_{K}^{\top} \boldsymbol{H}_{K}\right)^{-1} \boldsymbol{H}_{K}^{\top} \overline{\boldsymbol{g}} \text {. }
$$

To find the best order model in FS expansion the gait cycle is modeled for $K \in[1, \ldots, 25]$. Then, the FS coefficients, for each $K$ are estimated and further evaluated by two well-known model selection criteria: Akaike information criterion (AIC) and Bayesian information criterion (BIC). Both AIC and BIC add a 


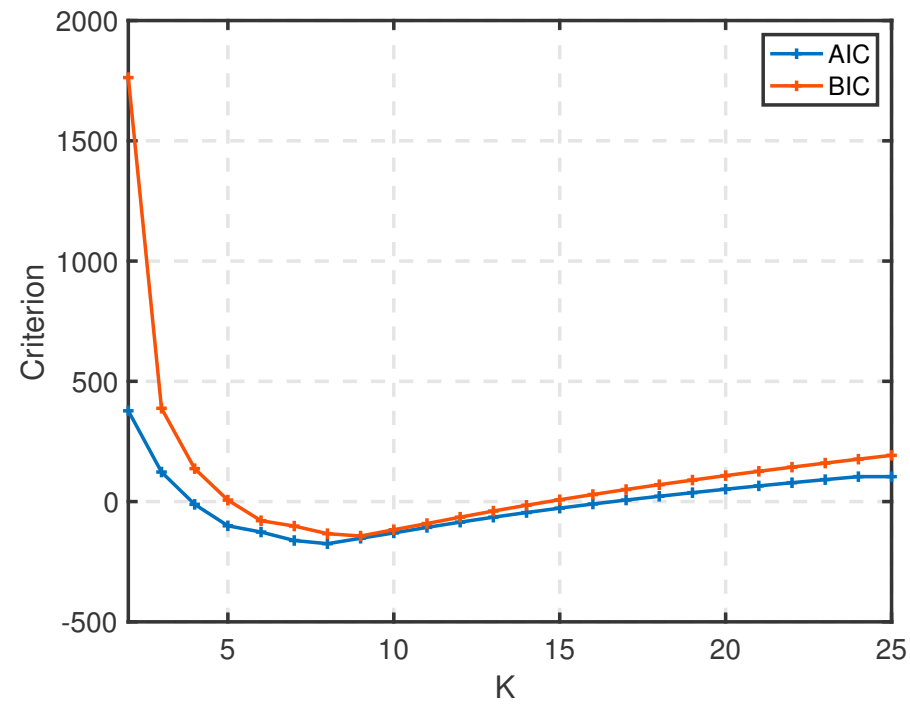

Figure 6.3: Model order selection.

penalty term to their objective function. The difference between the two criteria is that BIC imposes a greater penalty for the number of parameters compared to AIC [28]. For a sample of size $L$, for each model order $K$, AIC and BIC are defined as

$$
\begin{aligned}
A I C & =-2 \log p\left(\overline{\boldsymbol{g}} \mid \hat{\boldsymbol{\theta}}_{K}\right)+2 K, \\
B I C & =-2 \log p\left(\overline{\boldsymbol{g}} \mid \hat{\boldsymbol{\theta}}_{K}\right)+K \log L,
\end{aligned}
$$

where $\mathrm{p}\left(\overline{\mathrm{g}} \mid \hat{\theta}_{\mathrm{K}}\right)=\mathcal{N}\left(\mathrm{H}_{\mathrm{K}} \hat{\theta}_{\mathrm{K}}, \mathrm{H}_{\mathrm{K}}\left(\mathrm{H}_{\mathrm{K}}^{\top} \mathrm{H}_{\mathrm{K}}\right)_{\mathrm{K}}^{-1} \mathrm{H}_{\mathrm{K}}^{\top}\right)$.

As Figure 6.3 suggests, $K=8$ and $K=9$ are the suitable model orders for the gait segments presented in Figure 6.1 according to AIC and BIC, respectively. While either of the two orders could be selected as the suitable model order for this scenario, we select the lower order, $K=8$. Hence, the corresponding signatures can be generated by only incorporating $2 K=16$ estimated coefficients of the FS, $\hat{\theta}$, into (6.11), as shown in Figure 6.4a. Figure 6.4b presents the gait signature $\overline{\boldsymbol{g}}(\tau)$, and its $95 \%$ confidence bound. As this figure shows, the averaging error has quite wide variance.

To evaluate the encoded signal by the estimated FS coefficients with 16 components, the approximate error between $\hat{G}[l]$ and $\bar{g}(\tau)$ are computed and illustrated in Figure 6.5. As the result indicates, the approximation error is quite small with narrow confidence bounds. That is, the approximation error introduced by the low order FS expansion is negligible compared to the averaging error. 


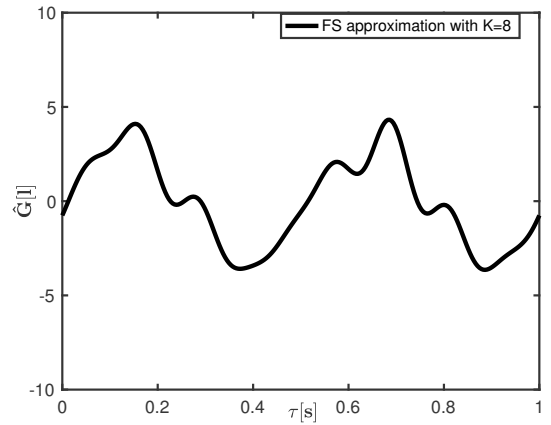

(a) FS approximate of the signature generated by (6.11) for $K=8$.

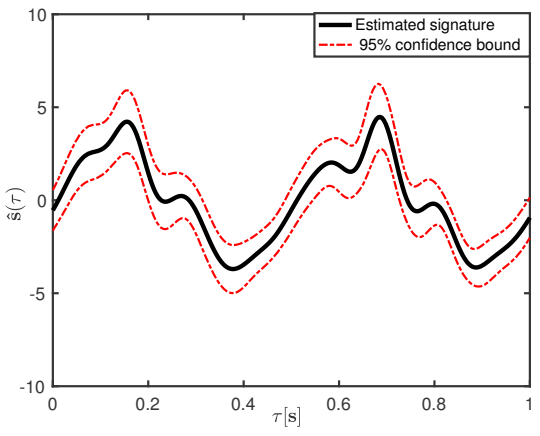

(b) Estimated signature using (6.4).

Figure 6.4: Comparison between the estimated signature and its FS expansion.

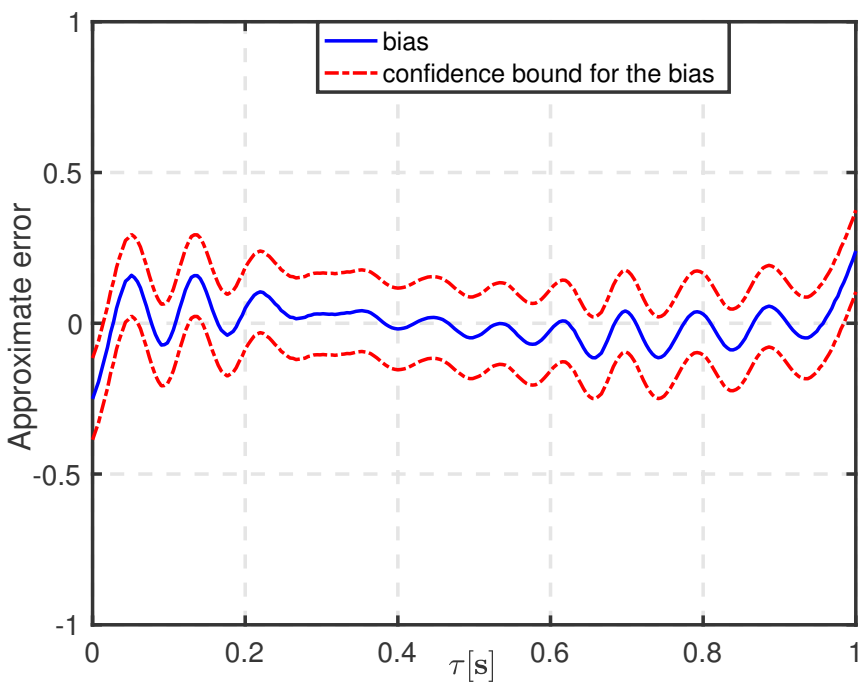

Figure 6.5: The approximation error and the 95\% confidence bound.

Subsequently, this low-dimensional feature vector together with the final least square cost value and the step time variations could provide a useful set of features for future classification purposes. 
Table 6.1: Experimental scenarios.

$\begin{array}{ccc}\text { Metion mode } & \begin{array}{c}\text { Walking } \\ \text { (W) }\end{array} & \begin{array}{c}\text { Running } \\ \text { (R) }\end{array} \\ \text { Fixed hand (1) } & \text { W1 } & \text { R1 } \\ \text { Swinging hand (2) } & \text { W2 } & \text { R2 } \\ \text { Pocket (3) } & \text { W3 } & \text { R3 } \\ \text { Backpack (4) } & \text { W4 } & \text { R4 }\end{array}$

\subsection{Experimental Results}

In this section, the proposed method introduced in Section 6.2 is evaluated on several experimental data. Additionally, the data collection setup, together with devices and all considered scenarios, is described in detail.

\subsubsection{Data Description}

In order to evaluate the performance of the proposed method, an extensive measurement campaign with different human motion modes and device poses has been designed. The sensor fusion Android app $[39,60]$ installed on a Nexus 5 was used to $\log$ accelerometer and gyroscope measurements with a sampling rate of $100 \mathrm{~Hz}$. All the measurements were collected over the same trajectory, which was $249 \mathrm{~m}$ in length with four sharp corners in a parking lot at Linköping University. Several subjects with different attributes (gender, height and weight) participated in the experiment. The data was collected for multiple human activities and device modes. Table 6.1 summarizes all the experimental scenarios. To simplify referring to each of these scenarios, Table 6.1 also assigns a specific symbol to each of them [49].

\subsubsection{Performance Evaluation}

As the first step, the gait cycles for all the scenarios are detected using Algorithm 4 and illustrated in Figure 6.2 together with estimated signatures which are computed based on (6.4).

Then, in order to fine-tune the gait segments extracted using the classical threshold-based algorithm, the proposed Algorithm 3 introduced in Section 6.2.1 is employed. The minimum and maximum of the most probable gait cycle times for each scenario, based on the histograms in Figure 6.2, are used as a reference to define the upper and lower bounds in the optimization problem. The bounds are set to $\epsilon_{l o}=0.5 \mathrm{~s}$ and $\epsilon_{u p}=1.4 \mathrm{~s}$.

Initial gait cycle times, obtained from Algorithm 4, are used to initialize the gait segmentation algorithm. Moreover, the initial signatures $\overline{\mathbf{g}}(\tau)$ to be used in Algorithm 3 are estimated by (6.4) using the initial gait cycle times. It is worth noting that after each iteration, the signature $\overline{\mathbf{g}}(\tau)$ will be updated by the tuned gait cycles. Figure 6.6 presents the evaluated cost function for eight iterations of the optimization progress considering all scenarios introduced in Table 5.1. As 


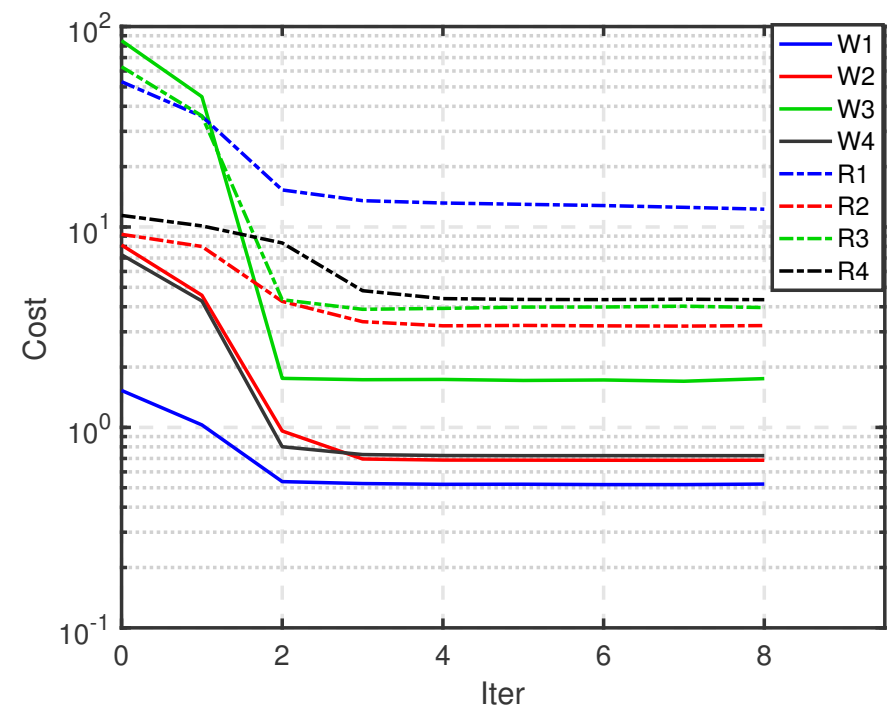

Figure 6.6: Evaluation $V\left(t_{0}: t_{M}\right)$ in (6.6) for eight iterations considering all scenarios in Table 6.1 .

the results indicate, in all scenarios after four iterations the cost function converges to the optimal values.

In order to evaluate how much the gait cycles are improved, the variance of all initial gait cycles with initial signature are compared with all optimal gait cycles with updated signatures. The distribution of the obtained variances is shown in Figure 6.7. The box levels are 5\%, 25\%, 50\%, 75\%, and 95\% quantiles and the asterisks show outlier values. As the figure suggests, for all scenarios the optimal variances are improved significantly and the mean of the variance of the optimal gait cycles is decreased notably. By comparing the walking mode in Figure 6.7a the running mode one in Figure $6.7 \mathrm{~b}$, it can be verified that running involves more unexpected movement for the device, hence there are more disturbances and it has a higher variance in total.

In order to give a better illustration of the performance of the proposed algorithm, we further examine "W2" and "R2" scenarios. The tuned gait cycles for these two scenarios are presented in Figure 6.8. Comparing the results with initial gait cycles, see Figure $6.2 \mathrm{c}$ and $6.2 \mathrm{~d}$, we realize that all the misdetected gait cycles are properly detected, as indicated in the histograms Figure $6.8 \mathrm{~b}$ and $6.8 \mathrm{~d}$, and all the gait cycles are perfectly tuned.

The updated gait signatures for all eight scenarios in Table 6.1, obtained from the tuned gait cycles, together with their corresponding 95\% confidence bounds, are presented in Figure 6.9. As the figures suggest, the estimated gait signatures for all considered scenarios have a unique pattern. For walking modes, all signatures have a very narrow confidence bound which is a verification that the tuned 


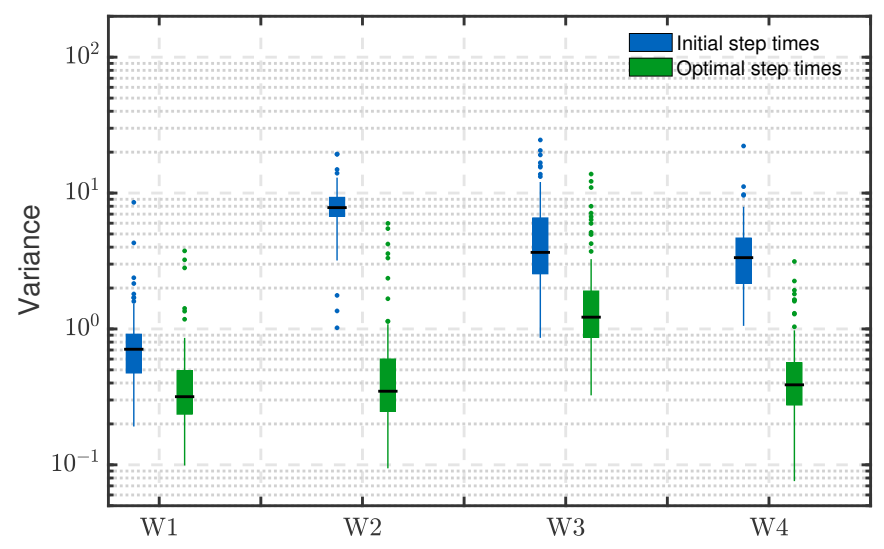

(a) Walking

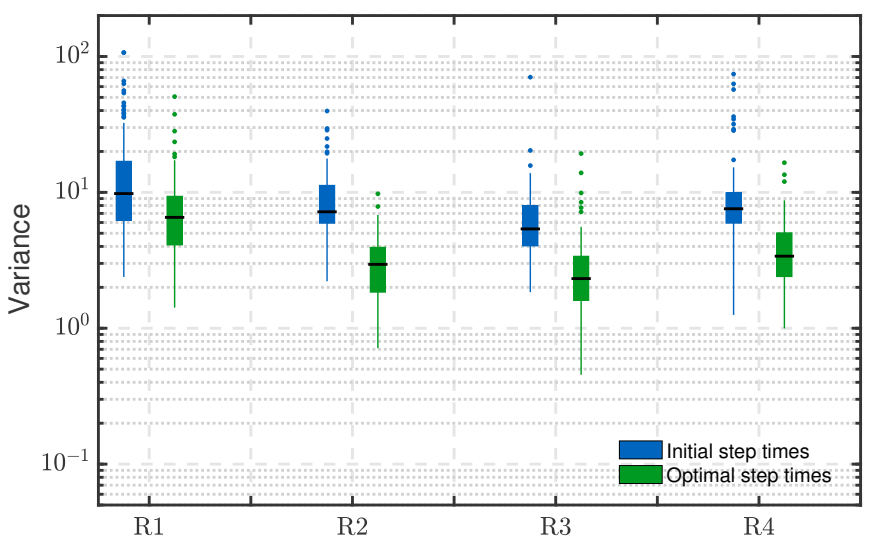

(b) Running

Figure 6.7: Variance of initial (blue) and tuned (green) gait cycles for all scenarios in Table 5.1 .

gait cycles resemble the final gait signatures. The bound for running mode, however, is wider especially for the fixed hand device modes. This can also be verified by Figure 6.7b, in which the variance of all optimal gait cycles for "R1" is higher than the other scenarios. This uncertainty can be explained by noting that users might have various unpredictable hand movements (especially while running) compared to the backpack and pocket scenarios in which the phone is more or less in a fixed position.

The updated signatures could be used for further investigation such as extract- 


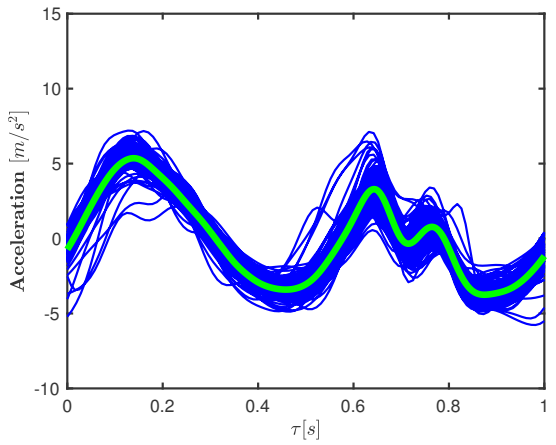

(a) W2 tuned gait cycles

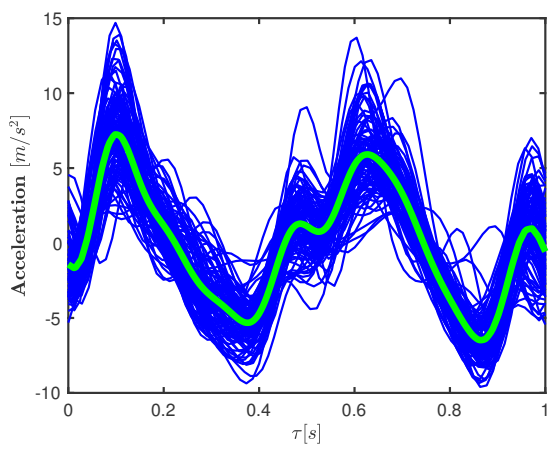

(c) R2 gait cycles

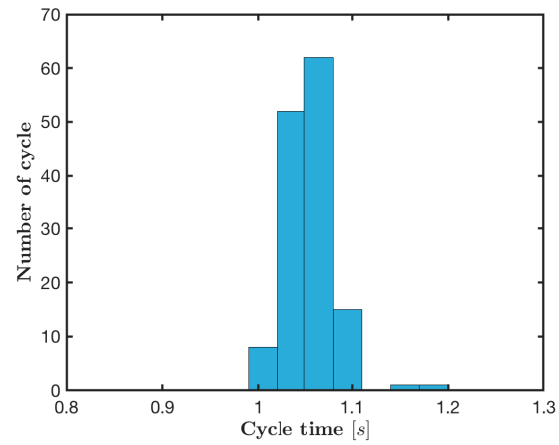

(b) W2 step time duration

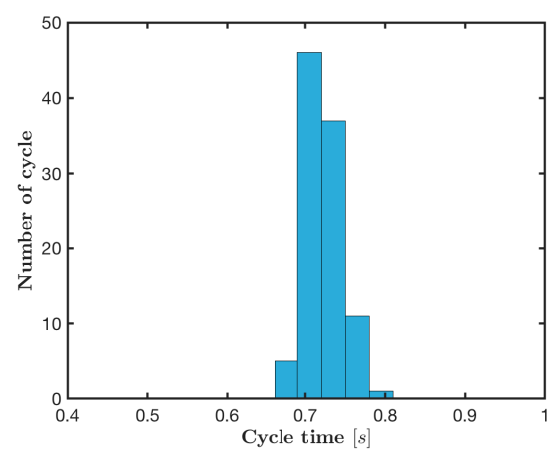

(d) R2 step time duration

Figure 6.8: Optimized gait cycles with updated signatures for "W2" and " $R 2$ " introduced scenarios in Table 6.1.

ing low-dimensional feature vectors for the gait cycles. Twenty different model orders are applied to all introduced scenarios to find the best model order for each of them. The Bayesian information criterion for all 20 model orders are presented in Figure 6.10. As shown in the figures, considering $K=8$ for walking and $K=10$ for running case would be suitable model orders to extract the feature vectors for the gait cycle in most cases, hence the signatures can be encoded efficiently. However, pocket mode in walking motion is the worst case from an FS perspective and requires $K=10$.

Finally, the uniqueness of the signatures can be verified by investigating the scalar product of of a pair of signatures. By doing so, it can be seen that the most unique signature corresponds to "W3" and that "R3" has the least similarity to the others. However, this is not always the case and there are cases that are not well separable from each other. For example, the signatures obtained for "W1", "W4" and "R2" have higher correlation to each other. 


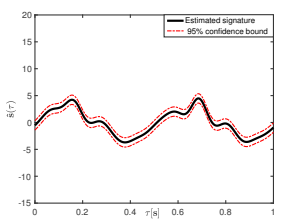

(a) $W 1$

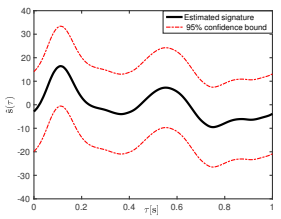

(e) R1

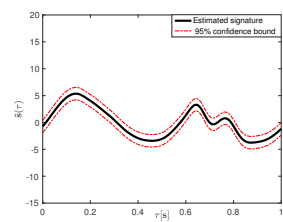

(b) $W 2$

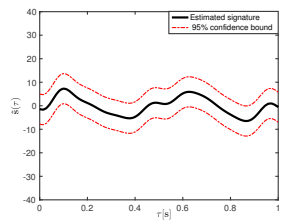

(f) $R 2$

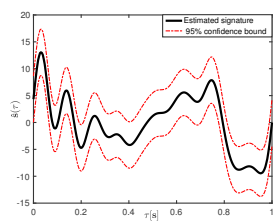

(c) $W 3$

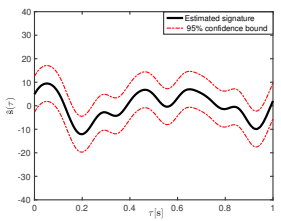

(g) $R 3$

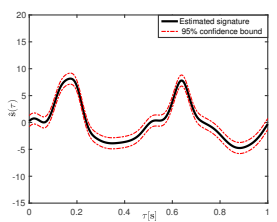

(d) $W 4$

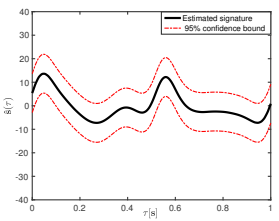

(h) $R 4$

Figure 6.9: Gait signatures for all eight scenarios introduced in Table 6.1 [top] walking, [bottom] running.

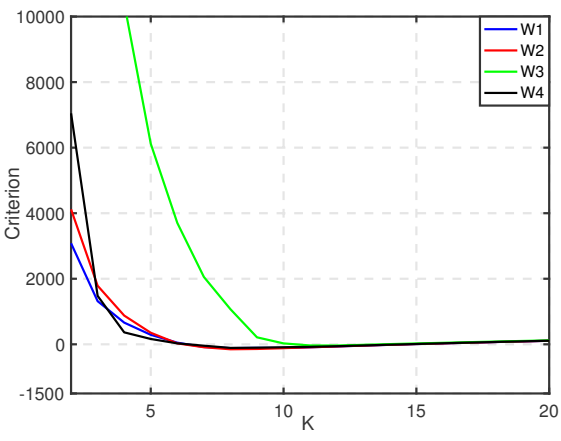

(a) Walking

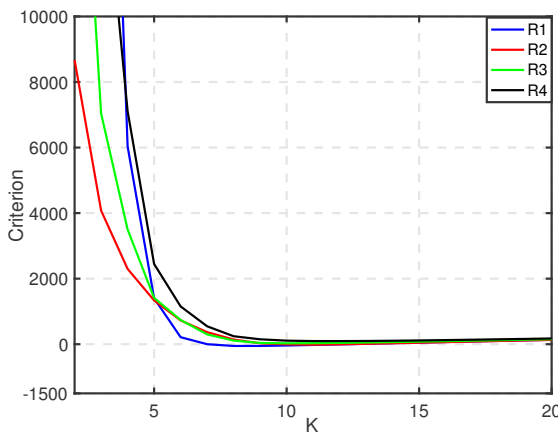

(b) Running

Figure 6.10: Model order selection using BIC.

One way to make the algorithm more automated is to classify each of the scenarios introduced in Table 6.1. For example, it is possible to consider the correlation of the gait signatures, given in Table 6.2 , as inputs to the classifier. That is, as a low hanging fruit, consider a classifier based on the highest correlation score. Chapter 7 studies this classification problem in detail and presents a classification framework with promising performance in terms of the classification 
Table 6.2: Correlation matrix for different signatures.

\begin{tabular}{|c|c|c|c|c|c|c|c|c|}
\hline & \multicolumn{8}{|c|}{ Classes } \\
\hline & $N^{1}$ & $\Delta^{2}$ & $\mathbb{S}^{3}$ & $N^{\star}$ & $\beta$ & קે & Pi & $\mathrm{s}^{\alpha}$ \\
\hline W1 & 1 & 0.81 & 0.46 & 0.84 & 0.58 & 0.82 & 0.26 & 0.47 \\
\hline W2 & 0.81 & 1 & 0.32 & 0.82 & 0.59 & 0.66 & 0.01 & 0.29 \\
\hline W3 & 0.46 & 0.32 & 1 & 0.43 & 0.32 & 0.44 & 0.39 & 0.39 \\
\hline W4 & 0.84 & 0.82 & 0.43 & 1 & 0.60 & 0.80 & 0.09 & 0.35 \\
\hline $\mathrm{R} 1$ & 0.5 & 0.59 & 0.32 & 0.60 & 1 & 0.68 & 0.19 & 0.66 \\
\hline $\mathrm{R} 2$ & 0.82 & 0.66 & 0.44 & 0.80 & 0.68 & 1 & 0.37 & 0.50 \\
\hline R3 & 0.26 & 0.01 & 0.39 & 0.09 & 0.19 & 0.37 & 1 & 0.57 \\
\hline $\mathrm{R} 4$ & 0.47 & 0.29 & 0.39 & 0.35 & 0.66 & 0.50 & 0.57 & 1 \\
\hline
\end{tabular}

accuracy.

\subsection{Conclusion}

Reliable pedestrian navigation systems require accurate step length estimation which in turn requires accurate gait cycle detection. In this work, an algorithm has been proposed for accurate gait cycle segmentation using IMU signals in multiple device and motion mode scenarios. For this purpose, we first used a classical thresholding algorithm to detect the gait cycles. Then, based on the asynchronous averaging of the gait cycles, a unique signature for each scenario was estimated. Furthermore, as a post-processing step, an optimization-based solution was proposed to tuned the segmentation of the IMU signals in a way that minimized the variance of signature for each gait cycle. We showed that a Fourier series expansion of gait signatures provides a low-dimensional feature vector which could possibly be highly beneficial together with the final least square cost value and the step time variations for classification purposes. The performance of the proposed method has been evaluated using measurements collected from IMUs embedded in smartphones for different motion modes while being carried with different device modes. The results indicate good performance for the gait cycle segmentation problem for all of the considered scenarios. 



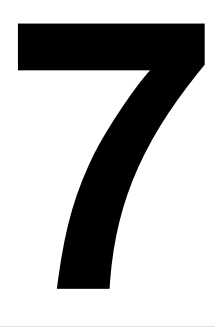

\section{Joint Pedestrian Motion State and Device Pose Classification}

This work is submitted to:

P. Kasebzadeh, K. Radnosrati, G. Hendeby, and F. Gustafsson. Joint pedestrian motion state and device pose classification. Submitted to IEEE Transactions on Instrumentation and Measurement, 2019b.

Novel features for joint classification of gait and device modes are proposed and multiple machine learning methods are adopted to jointly classify the modes. The classification accuracy as well as the $\mathrm{F}_{1}$ score of two standard classification algorithms, KNN and GP, are evaluated and compared against a proposed neural network based classifier, all introduced in Section 3.2. The proposed features are the correlation scores of a detected gait cycle relative to a set of unique gait signatures as well as the gait cycle time, all extracted from hand-held IMUs. In order to take the temporal correlation between classes into account, the coarse classifiers' estimates are fed into an HMM unit, presented in Section 2.4, to obtain the fine class estimates. The performance of the proposed method is evaluated on a large dataset including two classes of gait modes (walking and running) and four classes of device modes (fixed and face-up in the hand, swinging in the hand, in the pocket and in the backpack). The experimental results validate the reliability of the considered features and effectiveness of the HMM unit.

The remainder of this chapter is organized as follows: The problem is formulated in Section 7.1. The performance of the proposed methods is evaluated in Section 7.2, followed by concluding remarks on the work presented in Section 7.3.

\subsection{Problem Formulation}

The gait cycle classification procedure, from collecting data using IMUs to detection and classification of gait cycles, is performed in four steps. An overview of 
Table 7.1: Gait cycle classification overview

$\begin{array}{ccc} & \text { Input } & \text { Output } \\ \text { Gait Segmentation } & y\left(t_{1}: t_{i}\right) & \hat{\boldsymbol{g}}_{m}(\tau), \overline{\boldsymbol{g}}_{c}(\tau), T_{m} \\ \text { (Section 7.1.1) } & \hat{\boldsymbol{g}}_{m}(\tau), \overline{\boldsymbol{g}}_{c}(\tau)> & \boldsymbol{X}_{m} \\ \text { Feature Extraction } & T_{m} & \\ \text { (Section 7.1.2) } & \boldsymbol{X}_{m} & \hat{\mathcal{C}}_{m} \\ \text { Classification } & \hat{\mathcal{C}}_{m} & \hat{\mathcal{C}}_{m \mid m}, \hat{\mathcal{C}}_{m \mid M} \\ \text { (Section 3.2) } & & \end{array}$

all steps is summarized in Table 7.1.

Given $N$ measurements, we first find a segmentation of the signal, each segment to one full gait cycle. It is worth mentioning that a human gait cycle consists of two consecutive steps. Each step is defined as the period it takes for the right/left foot to toe off, while the other leg is on the ground and stationary, until the other foot toes off. This periodic behavior implies that the measured IMU signals from human activities should have a periodic component. In the rest of this work, we only use the norm of the accelerometer signals.

\subsubsection{Gait Segmentation}

In order to extract the pattern of the gait cycle with better quality, a preprocessing stage is applied to the raw measured data. In the pre-processing stage, the signal is filtered through a fourth-order band-pass Butterworth filter to attenuate all frequencies outside the band-pass and to obtain a clean signal. Depending on the application, different attributes of the clean signal can be used for classification purposes.

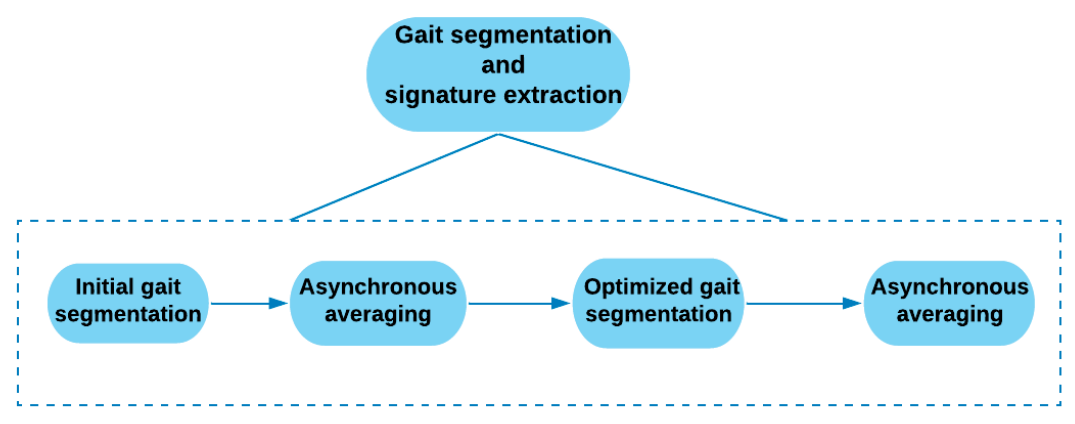

Figure 7.1: The flow diagram of the gait signature extraction using norm of pre-processed IMU signals. 


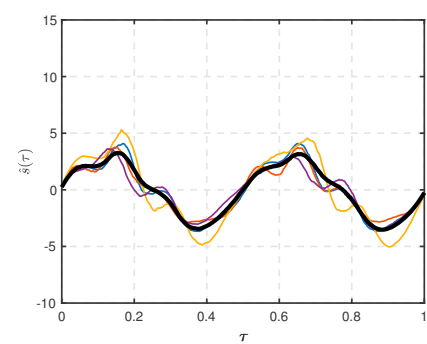

(a) W1

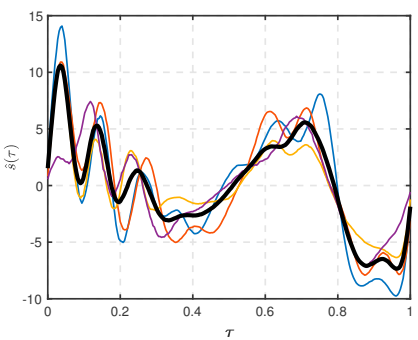

(c) $W 3$

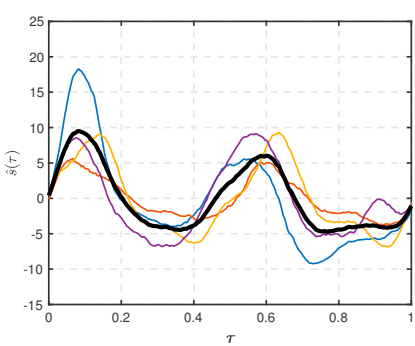

(e) R1

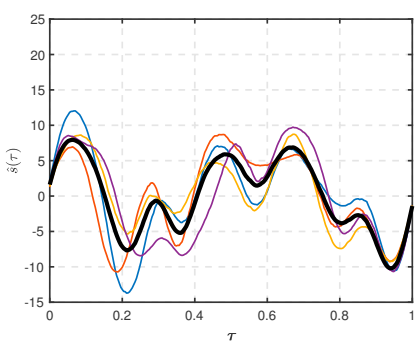

(g) $R 3$

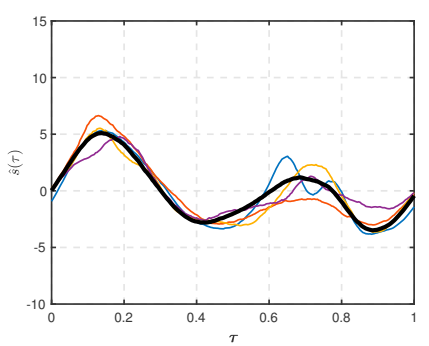

(b) $W 2$

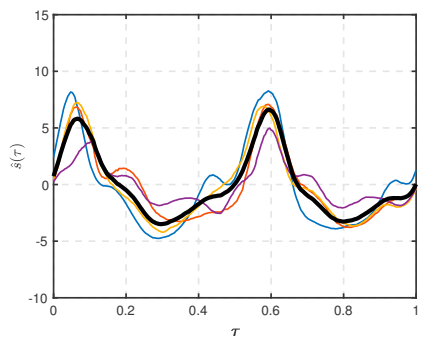

(d) $W 4$

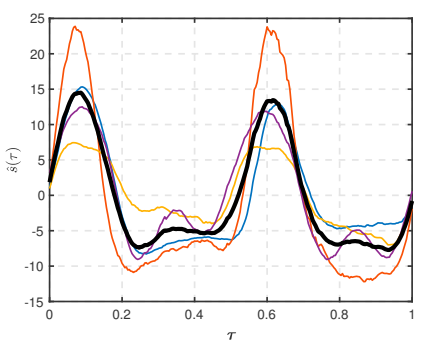

(f) $R 2$

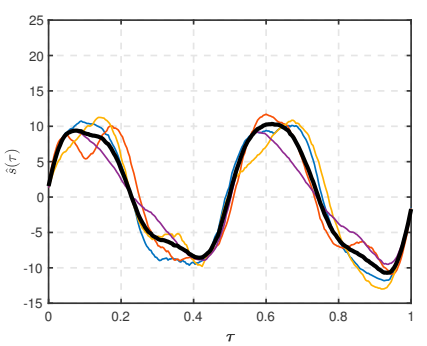

(h) $R 4$

Figure 7.2: Reference signals for four different individuals for all scenarios in Table 7.2. The bold solid line indicates the mean of all four reference signals which is considered as the signature for each case. 
Table 7.2: Experimental scenarios. For more information, see [49]. (Class label $c=1-8$ within parenthesis.)

$\begin{array}{ccc}\begin{array}{c}\text { Motion } \\ \text { mode }\end{array} & \begin{array}{c}\text { Walking } \\ (\mathbf{W})\end{array} & \begin{array}{c}\text { Running } \\ (\mathbf{R})\end{array} \\ \begin{array}{c}\text { Device mode } \\ \text { Fixed hand (1) }\end{array} & \mathrm{W} 1 /(1) & \mathrm{R} 1 /(5) \\ \begin{array}{c}\text { Swinging hand (2) } \\ \text { Pocket (3) }\end{array} & \mathrm{W} 2 /(2) & \mathrm{R} 2 /(6) \\ \text { Backpack (4) } & \mathrm{W} 3 /(3) & \mathrm{R} 3 /(7) \\ \text { W } 4 /(4) & \mathrm{R} 4 /(8)\end{array}$

The gait segmentation and analysis is summarized and depicted in Figure 7.1. For more information see Chapter 6.

The gait signatures obtained by applying this method to the experimental data of four individuals, explained in detail in Chapter 5, are given in Figure 7.2. As the figure suggests, each class can be represented using a unique signature.

\subsubsection{Feature Extraction}

In order to train the classifiers in this work, we use two novel characteristics of the detected gait cycles; the 8-dimensional correlation scores of each detected gait cycle relative to the gait signatures and the 1-dimensional gait cycle duration time. All in all, in this work, 9 features, $n_{f}=9$, are considered for classification purposes.

\section{Correlation Score}

The correlation scores are defined based on eight unique signatures of the reference signals corresponding to the scenarios introduced in Table 7.2. Define $\hat{\boldsymbol{g}}_{m}(\tau)$, as the $m:$ th detected gait cycle and $\overline{\boldsymbol{g}}_{c}(\tau), c \in\left\{1, \ldots, n_{c}\right\}$, as the signature of the $c:$ th class. The correlation score can be computed by

$$
f_{\text {corr }}\left(\hat{\boldsymbol{g}}_{m}, \overline{\boldsymbol{g}}_{c}\right)=\frac{\hat{\boldsymbol{g}}_{m}^{\top} \overline{\boldsymbol{g}}_{c}}{\sqrt{\left(\hat{\boldsymbol{g}}_{m}^{\top} \hat{\boldsymbol{g}}_{m}\right)\left(\overline{\boldsymbol{g}}_{c}^{\top} \overline{\boldsymbol{g}}_{c}\right)}} .
$$

In order to classify the gait cycles, we compute the correlation between each gait cycle $m$, and the 8 signatures. The obtained correlation value, corresponding to the $m$ :th gait cycle and the signature in the $c$ :th class, is then used as a feature in multiple classification algorithms. Given that, in this work, 8 different classes are considered, the correlation score results in an 8-dimensional feature vector, $n_{f}^{\text {corr }}=8$. One way to evaluate the uniqueness of the gait signatures is to compute their cross-correlations with each other. The correlation matrix corresponding to the correlation scores of the gait signatures are given in Table 7.3. As the table indicates, the cross-correlation values typically lie in the range $[0.6,0.85]$ while there are some classes with more similar signatures. Hence, the correlation 
Table 7.3: Correlation matrix for different signatures.

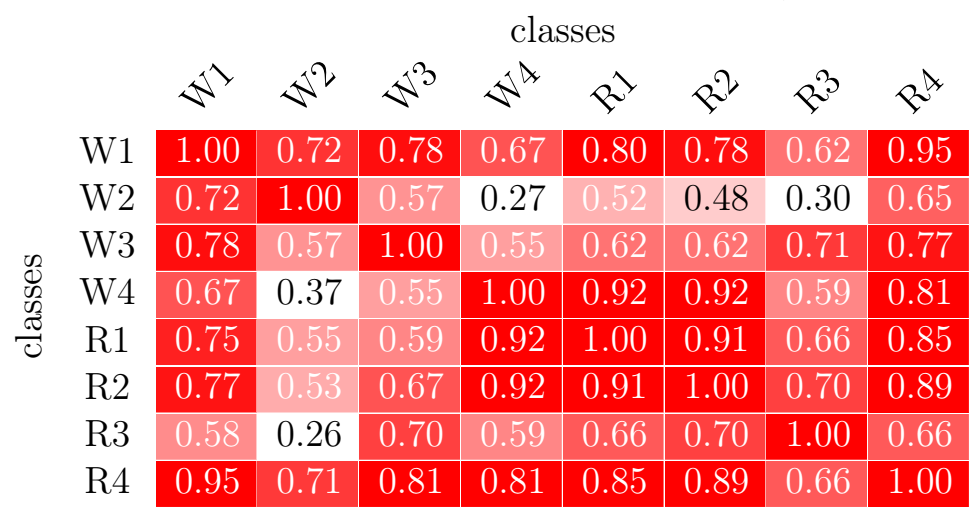

score has a great potential in forming feature vectors to be used in developing classifiers.

For example, as the simplest solution, one can solve the classification problem solely based on the highest correlation score. Let $\boldsymbol{X}_{m}^{\gamma}=f_{\text {corr }}\left(\hat{\boldsymbol{g}}_{m}, \overline{\boldsymbol{g}}_{c}\right) \in \mathbb{R}_{f}^{n_{f}^{\text {corr }}} \mathrm{de}$ note the feature vector containing the correlation scores computed by (7.1) for the $m$ :th gait cycle and the signature of the $c$ :th class. Using the highest correlation score approach, the class of the $m$ :th gait cycle is estimated as

$$
\hat{\mathcal{C}}_{m}=\underset{\gamma \in\left\{1, \ldots, n_{f}^{\text {corr }}\right\}}{\arg \max } \boldsymbol{X}_{m}^{\gamma},
$$

where $\hat{\mathcal{C}}_{m} \in\left\{1, \ldots, n_{c}\right\}$ is the index indicating that the $m$ :th gait cycle belongs to the $c$ :th class.

\section{Gait cycle time}

Different gait modes, walking and running, result in different gait cycle times. In order to consider the extra information obtained from the user's velocity, the gait cycle time is also considered as a feature, $\boldsymbol{X}_{m}^{T}$, in the classifiers. Although the gait cycle time is not of any use for device mode identification, it gives a clear decision boundary for the two motion modes.

Figure 7.3 shows histograms of all 8 signatures proving that the gait modes can be trivially identified by considering the gait cycle time. In the case of walking mode, the gait cycle time is typically between 1 and $1.2 \mathrm{~s}$, while for the running mode the cycle time interval mostly lies between 0.6 and $0.85 \mathrm{~s}$. Hence, this feature is very beneficial to minimize the misclassification between gait modes. 


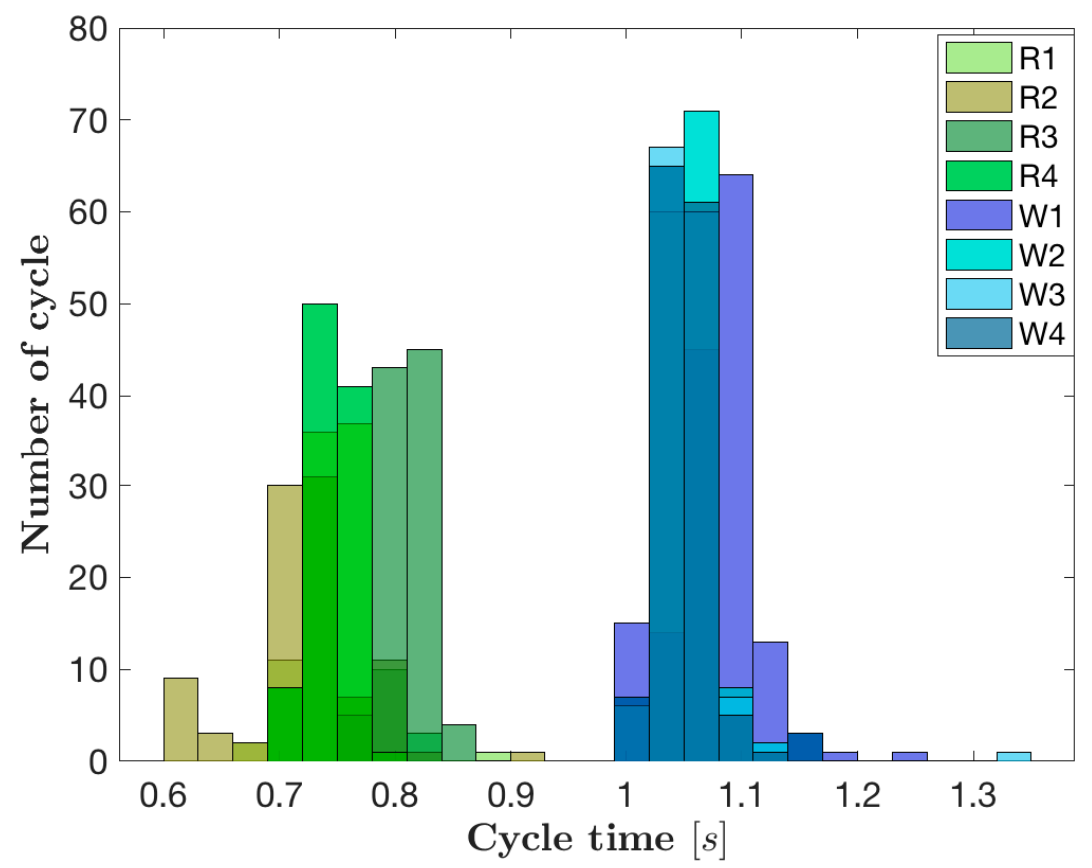

Figure 7.3: Histogram of gait cycle time for running and walking modes.

\subsubsection{Generating the Dataset}

The introduced 9-dimensional feature vector containing the correlation scores for all gait cycles, $\boldsymbol{X}^{\gamma} \in \mathbb{R}^{M \times n_{f}^{\text {corr }}}$, and the gait cycle times, $\boldsymbol{X}^{T} \in \mathbb{R}^{M \times n_{f}^{T}}$, are stacked together to make the input matrix $X \in \mathbb{R}^{M \times n_{f}}$. In this work, we divide the data into training set of size $n_{r}$, development set $n_{d}$, and test set $n_{e}$ with $n_{d}=n_{e}=\frac{M-n_{r}}{2}$. It is worth noting that the development set is used to crossvalidate the trained algorithms using classifiers introduced in Section 3.2 in order to avoid overfitting, underfitting or any other penalties that might increase the classification error of the test set.

The hyperparameters of the algorithms are all selected such that the development set error is minimized. Then, as the final step, the trained classifiers are applied to the test set (the part of the dataset which has not been seen during the training procedure) and the performance of the proposed algorithms is reported.

\subsection{Experimental Results}

In order to evaluate the performance of the proposed method, an extensive dataset with different human motion modes and device poses is considered [49].

A total number of 8900 gait cycles were collected, in two different campaigns, 
$80 \%$ of which were chosen for training. Since all the measurements were collected using the same device, they belong to the same underlying distribution. This allowed us to use the remaining $20 \%$ to extract equally-sized development and test sets. The first set of measurements was collected in a building at Twente University. During the experiments the subjects walked three different paths, with a mixture of different motion modes. The second campaign was performed over the same trajectory, which was $249 \mathrm{~m}$ in length with four sharp corners in a parking lot at Linköping University. Several subjects with different attributes (gender, height and weight) participated in the experiment.

In the rest of this section, we first evaluate the coarse classification performance where the temporal coherence in the consecutive classes are not considered. Then, the fine classification performances are provided in which the HMM smoother is adapted to smooth the class probability estimations. Performance is evaluated in terms of the confusion matrix, classification accuracy and the $F_{1}$ score.

Let $n_{t p}$ and $n_{t n}$ denote the number of true positives and true negatives, respectively. Assuming that the total number of class memberships in the test set is given by the cardinality $\left|\mathcal{C}_{\text {test }}\right|$, the accuracy is given by $n_{t p}+n_{t n} /\left|\mathcal{C}_{\text {test }}\right|$. In order to compute the $\mathrm{F}_{1}$ score, we first find the per-class scores, $\mathrm{F}_{1}^{c}$, and then report their average over the 8 classes.

Define $n_{t p}^{c}$ and $n_{f p}^{c}$ as the number of true positives and false positives corresponding to the class $c \in[1, \ldots, 8]$, respectively. The per-class precision, $p^{c}$, which is the ratio of true positives to those predicted positive, and the recall, $v^{c}$, which is the ratio of true positives to all positives, are given by

$$
\begin{aligned}
& p^{c}=\frac{n_{t p}^{c}}{n_{t p}^{c}+n_{f p}^{c}}, \\
& v^{c}=\frac{n_{t p}^{c}}{n_{t p}^{c}+n_{f n}^{c}},
\end{aligned}
$$

where $n_{f n}^{c}$ is the number of false negatives corresponding to class $c$. The per-class $\mathrm{F}_{1}$ score that weights precision and recall equally is given by

$$
F_{1}^{c}=\frac{2 p^{c} v^{c}}{p^{c}+v^{c}}
$$

\subsubsection{Coarse Classification}

The KNN and GP classifiers were trained using 5-fold cross validation using the training and development datasets. For each classifier, different hyperparameters were evaluated and their performances were compared. The best results using the KNN classifier was obtained when 10 nearest neighbors were considered. Table 7.4 presents the confusion matrix computed for the coarse KNN classifier relative to the true class labels $\mathcal{C}_{e}$. The classification accuracy obtained using the $\mathrm{KNN}$ method is $82 \%$ and the $F_{1}$ score is 0.79 . 
The trained GP classifier is based on the squared exponential kernel whose hyperparameters are $\sigma_{f}=1$ and $l=1$. The coarse classification confusion matrix using GP is provided in Table 7.5. As expected, GP improves the classification performance and reaches a classification accuracy of $83 \%$ and $F_{1}$ score of 0.80 .

Table 7.4: Coarse classification confusion matrix corresponding to the KNN classifier.

\begin{tabular}{|c|c|c|c|c|c|c|c|c|}
\hline & \multicolumn{8}{|c|}{ Classes } \\
\hline & $N^{\prime}$ & $a^{2}$ & $\mathbf{N}^{3}$ & $N^{\star}$ & E & $2^{2}$ & $B^{3}$ & $e^{*}$ \\
\hline W1 & 68 & 9 & 3 & 16 & 1 & 0 & 0 & 0 \\
\hline W2 & 6 & 81 & 1 & 3 & 0 & 0 & 0 & 0 \\
\hline W3 & 6 & 3 & 94 & 2 & 0 & 0 & 0 & 0 \\
\hline W4 & 20 & 6 & 2 & 79 & 0 & 0 & 0 & 0 \\
\hline $\mathrm{R} 1$ & 0 & 0 & 0 & 0 & 74 & 10 & 0 & 12 \\
\hline $\mathrm{R} 2$ & 0 & 1 & 0 & 0 & 8 & 79 & 1 & 7 \\
\hline R3 & 0 & 0 & 0 & 0 & 0 & 1 & 96 & 1 \\
\hline $\mathrm{R} 4$ & 0 & 0 & 0 & 0 & 17 & 10 & 3 & 80 \\
\hline
\end{tabular}

In order to tune the neural network, we first ignore the regularization term in the cost function (3.14b) as well as the dropout technique. The main objective, at this stage, is to design a neural network with low bias, hence low training error rate, without considering the overfitting problem, denoted by the variance. The hyperparameters $\beta_{1}=0.9, \beta_{2}=0.999$ and $\epsilon=10^{-8}$ of the Adam optimization algorithm were chosen as recommended by [53]. Then, the dropout and regularization methods are activated to reduce the variance between the train and test error rates.

In order to find the number of hidden layers and hidden units in each layer, a range of different candidates was evaluated. The best results were achieved using a 4-layer neural network that can be defined by a $9-450-250-150-8$ structure. All the hyperparameters of the developed neural network are summarized in Table 7.7.

The confusion matrix corresponding to the proposed neural network coarse classification is reported in Table 7.6. The performance metrics are improved compared to both KNN and GP classifiers with a classification accuracy of $91 \%$ and $F_{1}$ score of 0.89 . Using mini-batches of size 256 , the cost values during the whole training phase are also presented in Figure 7.4. Although the cost function has a decreasing trend over the whole training phase, as a result of using minibatch optimization, during each epoch, the cost values might even increase for some mini-batches.

As a result of governing the gait cycle time feature, even the simplest KNN classifier is able to find a very good decision boundary between the two gait modes. However, this situation does not hold for the device modes within each of the two gait modes and the coarse class estimates of the classifiers have different degrees of misclassifications. The W3 and R3 classes, corresponding to walking and 
Table 7.5: Coarse classification confusion matrix corresponding to the GP classifier.

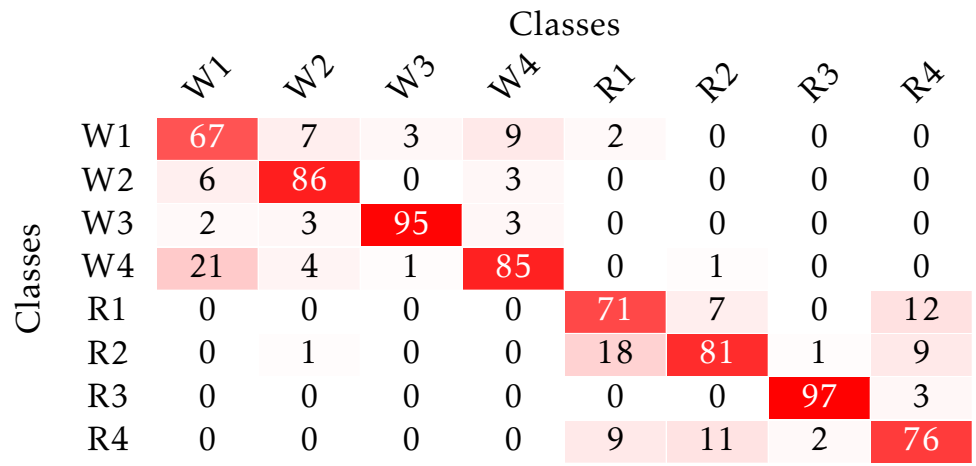

Table 7.6: Coarse classification confusion matrix corresponding to the proposed neural network classifier.

\begin{tabular}{|c|c|c|c|c|c|c|c|c|}
\hline & \multicolumn{8}{|c|}{ Classes } \\
\hline & 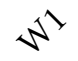 & $\mathbf{N}^{2}$ & $N^{3}$ & $N^{\star}$ & से & $\mathbf{p}^{2}$ & $R^{3}$ & $e^{*}$ \\
\hline W1 & 88 & 3 & 0 & 12 & 0 & 0 & 0 & 0 \\
\hline W2 & 3 & 95 & 1 & 12 & 0 & 0 & 0 & 0 \\
\hline W3 & 1 & 0 & 99 & 0 & 0 & 0 & 0 & 0 \\
\hline W4 & 7 & 2 & 0 & 86 & 0 & 0 & 0 & 0 \\
\hline $\mathrm{R} 1$ & 0 & 0 & 0 & 0 & 84 & 7 & 0 & 11 \\
\hline $\mathrm{R} 2$ & 1 & 0 & 0 & 0 & 3 & 92 & 3 & 3 \\
\hline R3 & 0 & 0 & 0 & 0 & 0 & 0 & 97 & 0 \\
\hline $\mathrm{R} 4$ & 0 & 0 & 0 & 0 & 13 & 1 & 0 & 86 \\
\hline
\end{tabular}

running with the device in pocket, are accurately estimated using all three methods. The accurate class estimates can be explained by considering the unique gait signatures of the W3 and R3 classes given in Figure 7.2. R1 and R4, on the other hand, are the most problematic classes, for which even the complex neural network classifier results in a misclassification rate of around $13 \%$. In the next section, we try to mitigate a portion of such misclassifications using the HMM smoothing stage.

\subsubsection{Fine Classification}

The outputs $\hat{\mathcal{C}}_{m}$ of all the classifiers are further processed and the filtering and smoothing estimates are evaluated using the test data. The fine-tuned estimated classes $\hat{\mathcal{C}}_{m \mid m}$, provided by the HMM block, are then compared to the true class labels and the same performance metrics as in the previous section are re-calculated. As expected, the KNN method has the poorest performance among the rest with a smoothing classification accuracy of around $86 \%$, which is 
Table 7.7: Hyperparameters of the proposed 4-layer neural network, with 8 units in the output layer.

\begin{tabular}{c|c|c|c|c|c|c|c}
$\begin{array}{c}\text { Hyper } \\
\text { parameter }\end{array}$ & $\beta_{1}$ & $\beta_{2}$ & $\epsilon$ & $\lambda$ & $\alpha$ & $\begin{array}{c}\text { Mini-batch } \\
\text { size }\end{array}$ & $\begin{array}{c}\text { Units per } \\
\text { hidden layer }\end{array}$ \\
\hline Values & 0.9 & 0.999 & $10^{-8}$ & 0.1 & $2 e^{-3}$ & 256 & $\begin{array}{l}n^{[1]}=450 \\
n^{[2]}=250 \\
n^{[3]}=150\end{array}$
\end{tabular}

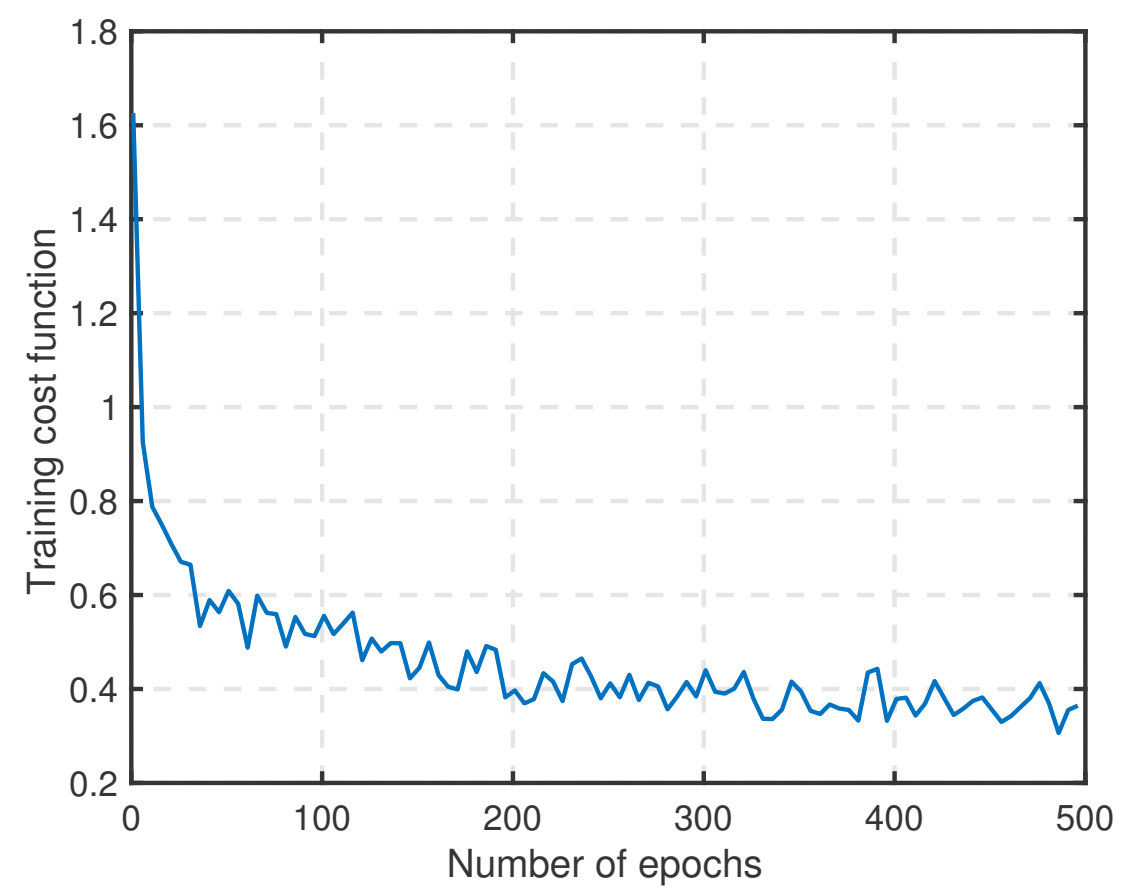

Figure 7.4: The training costs of the proposed neural network, obtained during training using Adam optimizer and back propagation technique.

less than the coarse estimates obtained from the neural network classifier. The confusion matrices for the filtered and the smoothed class estimates of the KNN method are not provided due to space limitations.

The GP classifier's accuracy improves from $83 \%$, obtained from the coarse estimates, to around $85 \%$ for filtering and $90 \%$ in the case of smoothing estimations. Hence, around 7\% classification accuracy can be gained by considering the temporal correlation in the GP classifier. The confusion matrices, for both filtering and smoothing, are given in Tables 7.8 and 7.9. Although the gained accuracy is non-negligible, the fine GP-based classifier still has a high rate of misclassification between fixed in hand and backpack device modes for both gait modes. 
Table 7.8: Filtered classification confusion matrix corresponding to the GP classifier.

\begin{tabular}{|c|c|c|c|c|c|c|c|c|}
\hline & \multicolumn{8}{|c|}{ Classes } \\
\hline & 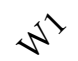 & $a^{2}$ & $N^{3}$ & $\Delta^{\star}$ & Pl & $p^{2}$ & $R^{3}$ & $E^{x}$ \\
\hline W1 & 76 & 10 & 2 & 10 & 7 & 0 & 0 & 0 \\
\hline W2 & 3 & 84 & 0 & 0 & 0 & 0 & 0 & 1 \\
\hline W3 & 3 & 5 & 93 & 4 & 0 & 0 & 0 & 0 \\
\hline W4 & 17 & 0 & 2 & 87 & 0 & 2 & 0 & 0 \\
\hline $\mathrm{R} 1$ & 0 & 0 & 0 & 0 & 84 & 1 & 0 & 14 \\
\hline $\mathrm{R} 2$ & 0 & 1 & 0 & 0 & 7 & 95 & 0 & 10 \\
\hline R3 & 0 & 0 & 3 & 0 & 0 & 1 & 97 & 2 \\
\hline $\mathrm{R} 4$ & 0 & 0 & 0 & 0 & 3 & 1 & 3 & 73 \\
\hline
\end{tabular}

Table 7.9: Smoothened classification confusion matrix corresponding to the GP classifier.

\begin{tabular}{|c|c|c|c|c|c|c|c|c|}
\hline & \multicolumn{8}{|c|}{ Classes } \\
\hline & $\sqrt{1}$ & $\Delta^{2}$ & $\mathbf{N}^{3}$ & $N^{*}$ & \& & $p^{2}$ & $p^{3}$ & $e^{*}$ \\
\hline W1 & 86 & 5 & 2 & 3 & 6 & 0 & 0 & 0 \\
\hline W2 & 0 & 91 & 0 & 0 & 0 & 0 & 0 & 0 \\
\hline W3 & 2 & 4 & 97 & 1 & 0 & 0 & 0 & 0 \\
\hline W4 & 11 & 0 & 0 & 96 & 0 & 1 & 0 & 0 \\
\hline $\mathrm{R} 1$ & 0 & 0 & 0 & 0 & 94 & 2 & 0 & 16 \\
\hline R2 & 0 & 0 & 0 & 0 & 0 & 97 & 1 & 8 \\
\hline R3 & 0 & 0 & 1 & 0 & 0 & 0 & 99 & 3 \\
\hline $\mathrm{R} 4$ & 0 & 0 & 0 & 0 & 0 & 0 & 0 & 73 \\
\hline
\end{tabular}

Table 7.10: Filtered classification confusion matrix corresponding to the proposed neural network classifier.

\begin{tabular}{|c|c|c|c|c|c|c|c|c|}
\hline & \multicolumn{8}{|c|}{ Classes } \\
\hline & $\$$ & $\$^{2}$ & $\$^{3}$ & $s^{*}$ & s & $\alpha^{2}$ & $R^{3}$ & $e^{*}$ \\
\hline W1 & 94 & 1 & 0 & 6 & 0 & 0 & 0 & 0 \\
\hline W2 & 3 & 96 & 0 & 0 & 0 & 0 & 0 & 0 \\
\hline W3 & 0 & 2 & 100 & 0 & 0 & 0 & 0 & 0 \\
\hline W4 & 4 & 0 & 0 & 94 & 0 & 0 & 5 & 0 \\
\hline R1 & 0 & 0 & 0 & 0 & 94 & 2 & 0 & 8 \\
\hline R2 & 0 & 0 & 0 & 0 & 6 & 94 & 1 & 0 \\
\hline R3 & 0 & 0 & 0 & 0 & 0 & 0 & 94 & 0 \\
\hline R4 & 0 & 0 & 0 & 0 & 0 & 4 & 0 & 92 \\
\hline
\end{tabular}


Table 7.11: Smoothened classification confusion matrix corresponding to the proposed neural network classifier.

\begin{tabular}{|c|c|c|c|c|c|c|c|c|}
\hline & & & & & & & & \\
\hline & $\$$ & $s^{2}$ & $\sqrt{3}^{3}$ & $s^{\Delta}$ & R & $e^{2}$ & $R^{3}$ & $p^{*}$ \\
\hline W1 & 98 & 0 & 0 & 0 & 0 & 0 & 0 & 0 \\
\hline W2 & 2 & 100 & 0 & 1 & 0 & 0 & 0 & 0 \\
\hline W3 & 0 & 0 & 100 & 0 & 0 & 0 & 0 & 0 \\
\hline W4 & 0 & 0 & 0 & 99 & 0 & 0 & 0 & 0 \\
\hline R1 & 0 & 0 & 0 & 0 & 100 & 0 & 0 & 8 \\
\hline R2 & 0 & 0 & 0 & 0 & 0 & 100 & 0 & 0 \\
\hline R3 & 0 & 0 & 0 & 0 & 0 & 0 & 100 & 0 \\
\hline $\mathrm{R} 4$ & 0 & 0 & 0 & 0 & 0 & 0 & 0 & \\
\hline
\end{tabular}

The best classification performance is obtained by smoothing the proposed neural network coarse classifier using the HMM block. As the confusion matrices provided in Tables 7.10 and 7.11 suggest, all the different scenarios of the joint gait mode and device mode classification problem can be distinguished highly accurately. Smoothing class estimates using the neural network classifier results in $100 \%$ accuracy for five out of eight classes. Although the most problematic case is still finding an appropriate classification boundary between R1-R4, the misclassification error is halved from $16 \%$ for GP to $8 \%$ for neural network. Figure 7.5 compares all the discussed classification methods in terms of the classification accuracy. As a general trend, using the additional smoothing stage improves the classification accuracy by an order of around $4 \%$ for KNN, and 7\% for GP and neural network. Finally, Table 7.12 summarizes the considered performance metrics achieved for all class estimates using the three classifiers (no HMM, filtered and smoothened).

Finally, the performance of the introduced approach in this work is also compared with Table III in [102]. In the reported table, walking and running motion mode together with 4 different device modes (texting, phone, pocket and swinging) are considered. Based on the provided description, the "texting" pose is the same as the fixed in hand device mode in our study. Since the number of classes

Table 7.12: Summary of the performance metrics, reported in the form of accuracy (percentage) / $F_{1}$ score, achieved for the three estimation methods using the three classifiers.

\begin{tabular}{|c|c|c|c|}
\hline $\begin{array}{l}\text { Estimation method } \\
\text { Classifier }\end{array}$ & Coarse & Filtered & Smoothened \\
\hline KNN & $82 / 0.79$ & $83 / 0.80$ & $86 / 0.82$ \\
\hline GP & $83 / 0.80$ & $85 / 0.81$ & $90 / 0.85$ \\
\hline NN & $91 / 0.89$ & $95 / 0.94$ & $98 / 0.97$ \\
\hline
\end{tabular}




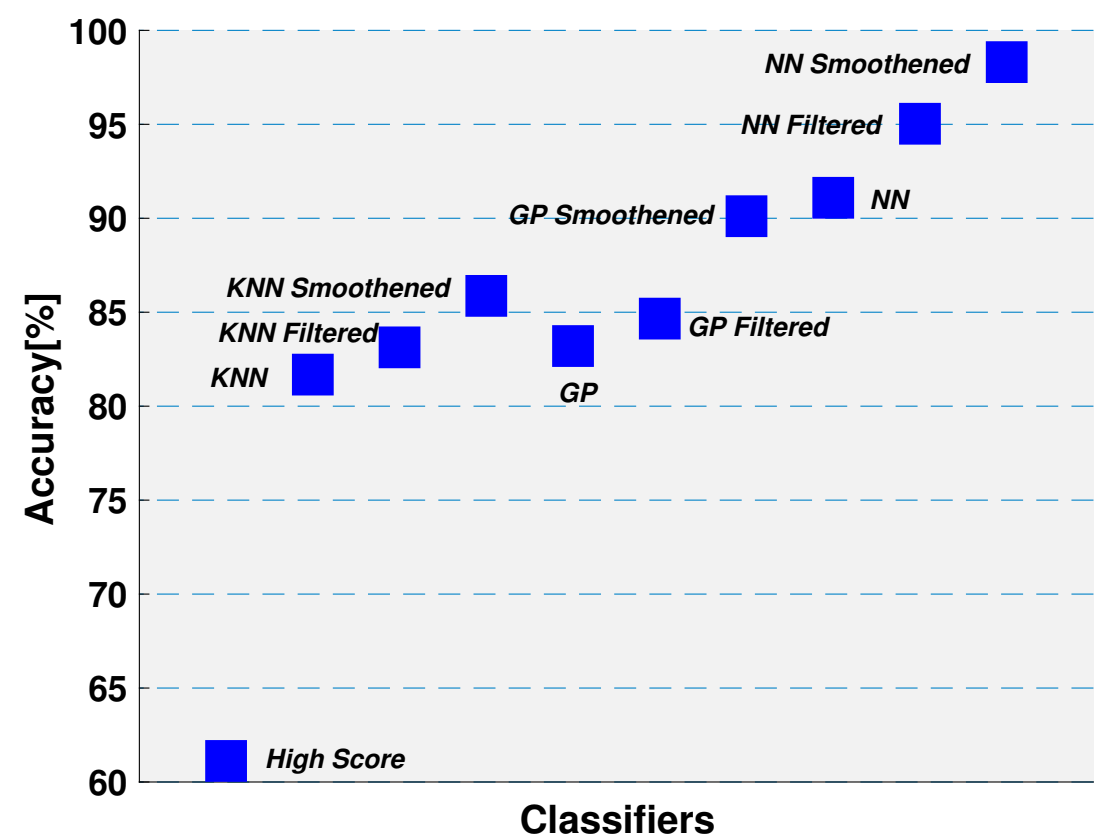

Figure 7.5: Comparison of the obtained classification accuracies for all discussed classifiers.

is the same in both studies, it is fair to compare the performance of the proposed method with the reported results in [102] for six common cases, all except W4 and R4.

The proposed method results in better performance, in terms of classification accuracy, for all of the considered six scenarios in [102]. More specifically, the classification accuracy for all running scenarios is considerably higher than what is achieved in the aforementioned study. Moreover, the highest achievable accuracy of the walking scenarios, as reported in [102], belongs to W1 with $94.8 \%$ accuracy. The method proposed in this work, however, results in slightly more accurate results with $98 \%$ classification accuracy for the same scenario W1.

\subsection{Conclusions}

The joint classification problem of the user's motion profile and the handheld device's carrying mode using IMU measurements was studied. Eight different classes, based on two different motion modes (walking and running) and four device modes (fixed and face-up in hand, swinging in hand, in the pocket and in the backpack) were considered. K-nearest neighbor, Gaussian process and feed forward neural network classifiers were adopted to jointly classify the aforemen- 
tioned classes. A set of novel classification features was derived from the preprocessed IMU measurements. Noting that there is a strong correlation between the classes over time, the classifiers' estimated output was further smoothened using an HMM unit. The highest classification accuracy was achieved by smoothing the neural network classifier's estimates using the HMM unit. Comparing the obtained results with a similar study validated the merit of the proposed method. 
Part III

\section{Conclusion and Future Work}





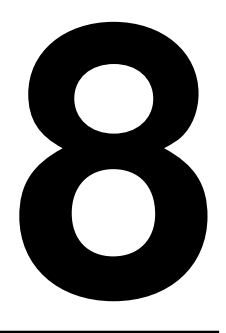

\section{Summary and Future Work}

Different aspects of pedestrian navigation, with an emphasis on GNSS-denied environments, are investigated in this thesis. To be more specific, the problem of human gait parameter learning which results in an improved dead-reckoning algorithm is studied. To do so, different parametric and non-parametric approaches are employed for gait modeling and classification.

The learning problem, formulated in terms of state-space models, relies on Bayesian inference to estimate the gait parameters. For the classification problem, different classifiers from a simple k-nearest neighborhood to a complex feed forward neural network with back propagation are considered.

The performance of the developed methods are evaluated on simulated data as well as an extensive dataset. In the rest of this chapter, a summary of the contributions is provided in Section 8.1 and then the thesis is finalized with possible future research directions in Section 8.2.

\subsection{Summary of Contribution}

Noting that accurate step detection is crucial in pedestrian dead reckoning applications, the first contribution of this thesis is to propose a filtering framework that learns the gait parameters when external position information is available. The approach proposed in Chapter 4 is based on a multi-rate Kalman filter bank that estimates the gait parameters, such as step length and step detection threshold. The learning process is performed by fusing different sensors, e.g. accelerometer, gyroscope with GPS which improves the PDR in time intervals when the position measurements are unavailable. It is shown that using a fixed threshold for step detection in the PDR algorithm might result in erroneous step length estimates. The obtained result indicates the merit of the proposed method, particularly when the user has a constant velocity motion. 
The dataset used for performance evaluations contains IMU measurements collected from several body-mounted and hand-held devices, as described in Section 5.2, and is used in different parts of this thesis, e.g. Section 5.3, Section 6.2, and Section 7.2. The whole dataset together with the ground truth trajectory is made available on-line. Individuals with different physical attributes participated in the data collection experiments and took three different trajectories. The second contribution, presented in Chapter 5, describes the detailed information of the data collection campaign and the hardware specifications. A few standard features that are used in the literature for activity mode classification applications based on IMU signals are extracted and the preliminary classification results are reported.

It is discussed that gait parameters are time-varying and individualdependent quantities. More specifically, depending on the individual's physical attributes, motion mode and the hand-held device's carrying mode, the optimal parameters might change. Hence, it is beneficial to define different classes for each of these modes.

The third contribution, presented in Chapter 6, strives to find a unique gait signature for some selected motion and device modes. The computed signatures are further modeled to compute a feature space to identify each of the considered modes. This method is also applied in a real world application with multiple motion and device mode combinations. It is shown that for any combination, it is possible to compute a unique gait signature. An initial classification analysis, based on the correlation between the computed signatures, is also performed to investigate the potential of the proposed features. The preliminary study shows the potential of the extracted signatures to be used as classification features.

The fourth contribution, presented in Chapter 7, takes one step further and proposes a two-step classification framework based on the computed features. A standard classifier, e.g. Gaussian process or feed forward neural network, is employed in the first step of the proposed method to compute initial class estimates. In the second step, the estimates are further refined by considering the inherent temporal correlation between the classes. The filtering and smoothing class estimates of different classifiers are then presented and their performances are compared against each other. It is shown that the proposed two-stage multi-class classification method can be used to accurately identify the motion and device mode.

\subsection{Future Work}

The main objective of this thesis is to propose solutions for human gait learning from uncertain IMU measurements. The learned gait parameters can be used in pedestrian navigation systems. The considered scenarios are relevant for hybrid indoor-outdoor positioning applications and can be used by collaborators. The proposed methods are the author's solution towards the existing problems. However, several research directions remain for further studies.

We have here presented algorithms for analyzing the gait as an enabling tech- 
nology. There are numerous applications that can benefit from this work. The most nearby illustration is the kind of step counters you can find in your smartphones and smart watches, which can differ a factor of two compared in the same situation. Also, the motion mode provided in Android and iOS often fails and can, for instance, decide cycling when walking. Certainly, there is room for improvement in the commercial solutions today.

We have mentioned applications to health monitoring, collapse detection, monitoring athletics activity, animal activity tracking, etc. The method of synchronous averaging we have proposed to represent the gait cycle is a very promising enabler for new studies in these areas. In particular, the low dimensional Fourier series expansion vector is quite appropriate for learning applications. This methodology has applications beyond human gait analysis, and can be used in many kind of health and construction monitoring applications where almost periodical sensor signals are used today to detect anomalies. One recent application we have applied the methodology to is monitoring mechanical heart pumps for possible mechanical faults or reduced capacity due to thrombosis. 



\section{Bibliography}

[1] IMU Dataset for Device and Motion Mode Classification. URL http: / / users.isy.liu.se/rt/parka23/research.html.

[2] Senion AB. Senion, 2017. URL https://goo.gl/NjVkk2.

[3] A. Akl, C. Feng, and S. Valaee. A novel accelerometer-based gesture recognition system. IEEE Sensors Journal, 59(12):6197-6205, December 2011.

[4] M. A. Al-Ammar, S. Alhadhrami, A. Al-Salman, A. Alarifi, H. S. Al-Khalifa, A. Alnafessah, and M. Alsaleh. Comparative survey of indoor positioning technologies, techniques, and algorithms. In International Conference on Cyberworlds (CW), Santander, Spain, October 2014.

[5] J. C. Alvarez, D. Alvarez, A. López, and R. C. González. Pedestrian navigation based on a waist-worn inertial sensor. Sensors, 12(8):10536-10549, 2012.

[6] B. D. Anderson and J. B. Moore. Optimal Filtering. Prentice Hall, Inc, 1979. ISBN 0-13-638122-7.

[7] P. Andersson. Adaptive forgetting in recursive identification through multiple models. Analysis and Optimization of Systems, 62:171-185, 1984.

[8] K. J. Åström. Introduction to Stochastic Control Theory. Dover Publications, Mineola, NY, USA, 2006.

[9] Y. Bar-Shalom, X. R. Li, and T. Kirubarajan. Estimation with Applications to Tracking and Navigation. Wiley-Interscience, 2001.

[10] Y. Bar-Shalom, S. Challa, and H. A. P. Blom. IMM estimator versus optimal estimator for hybrid systems. IEEE Transactions on Aerospace and Electronic Systems, 41(3):986-991, July 2005.

[11] M. Basso, M. Galanti, G. Innocenti, and D. Miceli. Pedestrian dead reckoning based on frequency self-synchronization and body kinematics. IEEE Sensors Journal, 17(2):534-545, January 2017. 
[12] L. E. Baum. An inequality and associated maximization technique in statistical estimation for probabilistic functions of Markov processes. In Inequalities III: Proceedings of the Third Symposium on Inequalities, pages 1-8, University of California, Los Angeles, 1972.

[13] S. Beauregard. Omnidirectional pedestrian navigation for first responders. In 4th Workshop on Positioning, Navigation and Communication (WPNC), Hannover, Germany, March 2007.

[14] C. M. Bishop. Pattern Recognition and Machine Learning (Information Science and Statistics). Springer-Verlag, 2006.

[15] C. Blaiotta. Learning generative socially aware models of pedestrian motion. IEEE Robotics and Automation Letters, 4(4):3433-3440, October 2019.

[16] H. A. P. Blom and Y. Bar-Shalom. The interacting multiple model algorithm for systems with Markovian switching coefficients. IEEE Transactions on Automatic Control, 33(8):780-783, August 1988.

[17] M. Briers, A. Doucet, and S. Maskell. Smoothing algorithms for state space models. Annals of the instittue of statistical Mathematics, 62(1):61-69, February 2010.

[18] K. Brzostowski. Toward the unaided estimation of human walking speed based on sparse modeling. IEEE Transactions on Instrumentation and Measurement, 67(6):1389 - 1398, February 2018.

[19] Jonas Callmer. Autonomous Localization in Unknown Environments. PhD thesis, Division of Automatic Control, Department of Electrical Engineering, Linköping University, 2013.

[20] C. Caramia, D. Torricelli, M. Schmid, A. M. Gonzalez, G. J. Vargas, F. Grandas, and J. L. Pons. IMU-based classification of Parkinson's disease from gait: A sensitivity analysis on sensor location and feature selection. IEEE Journal of Biomedical and Health Informatics, 22(6):1765 - 1774, August 2018.

[21] Z. Chen, Q. Zhu, Y. C. Soh, and L. Zhang. Robust human activity recognition using smartphone sensors via CT-PCA and online SVM. IEEE Transactions on Industrial Informatics, 13(6):3070-3080, December 2017.

[22] A. K. Chowdhury, D. Tjondronegoro, V. Chandran, and S. G. Trost. Physical activity recognition using posterior-adapted class-based fusion of multiaccelerometer data. IEEE Journal of Biomedical and Health Informatics, 22 (3):678-685, May 2018.

[23] M. Cornacchia, K. Ozcan, Y. Zheng, and S. Velipasalar. A survey on activity detection and classification using wearable sensors. IEEE Sensors Journal, 17(2):386-403, January 2017. 
[24] P. Davidson and R. Piche. A survey of selected indoor positioning methods for smartphones. IEEE Communications Surveys \& Tutorials, 19(2):1347 1370, May 2017.

[25] L.E. Diez, A. Bahillo, J. Otegui, and T. Otim. Step length estimation methods based on inertial sensors: A review. IEEE Sensors Journal, 18(17):69086926, September 2018.

[26] M. Elhoushi, J. Georgy, A. Noureldin, and M. J. Korenberg. Motion mode recognition for indoor pedestrian navigation using portable devices. IEEE Transactions on Instrumentation and Measurement, 65(1):208-221, January 2016.

[27] M. Elhoushi, J. Georgy, A. Noureldin, and M. J. Korenberg. A survey on approaches of motion mode recognition using sensors. IEEE Transactions on Intelligent Transportation Systems, 18(7):1662-1686, July 2017.

[28] F. J. Fabozzi, S. M. Focardi, S. T. Rachev, and B. G. Arshanapalli. The Basics of Financial Econometrics: Tools, Concepts, and Asset Management Applications. John Wiley \& Sons, 2014.

[29] S. H. Fang, Y. X. Fei, Z. Xu, and Y. Tasao. Learning transportation modes from smartphone sensors based on deep neural network. IEEE Sensors Journal, 17(18):6111-6118, September 2017.

[30] T. Feigl, S. Kram, P. Woller, R. H. Siddiqui, M. Philippsen, and C. Mutschler. A Bidirectional LSTM for Estimating Dynamic Human Velocities from a Single IMU. In Proc. of the 10th International Conference on Indoor Positioning and Indoor Navigation (IPIN2019), Pisa, Italy, September 2019.

[31] E. Foxlin. Pedestrian tracking with shoe-mounted inertial sensors. IEEE Comput. Graphics Appl., 25:38-46, November 2005.

[32] GIS Geography. World geodetic system WGS84, 2017. URL https:// goo.gl/BgGVV7.

[33] Z. Ghahramani. Hidden Markov models. In An Introduction to Hidden Markov Models and Bayesian Networks, pages 9-42. World Scientific Publishing Co., Inc., River Edge, NJ, USA, 2002.

[34] GPS.Gov. Official U.S. government information about the global positioning system (GPS) and related topics, 2017. URL https://goo.gl/ t $6 \mathrm{P} \times \mathrm{bu}$.

[35] C. Gusenbauer, D. Isert and J. Krosche. Self-contained indoor positioning on off-the-shelf mobile devices. In Proceedings of IEEE 2nd Conference on Indoor Positioning and Indoor Navigation (IPIN2010), Zurich, Switzerland, September 2010. 
[36] F. Gustafsson. Statistical Sensor Fusion. Studentlitteratur, 2010.

[37] F. Gustafsson, F. Gunnarsson, N. Bergman, and U. Forssell. Particle filters for positioning, navigation and tracking. IEEE Transactions on Signal Processing, 50(2):425-437, February 2002.

[38] G. Hendeby. Performance and Implementation Aspects of Nonlinear Filtering. PhD thesis, Linköping Studies in Science and Technology, 2008.

[39] G. Hendeby, F. Gustafsson, and N. Wahlström. Teaching sensor fusion and Kalman filtering using a smartphone. In Proceedings of the 19th IFAC World Congress, Cape Town, South Africa, August 2014.

[40] G. Hendeby, F. Gustafsson, N. Wahlström, and S. Gunnarsson. Platform for teaching sensor fusion using a smartphone. International journal of engineering education, 33(2):781-789, December 2017.

[41] N. Ho, P. Truong, and G. Jeong. Step-detection and adaptive step-length estimation for pedestrian dead-reckoning at various walking speeds using a smartphone. Sensors, 16(9):1-13, September 2016.

[42] A. H. Jazwinski. Stochastic processes and filtering theory, volume 64. Academic Press, Inc., 1970.

[43] R. Jirawimut, P. Ptasinski, V. Garaj, F. Cecelja, and W. Balachandran. A method for dead reckoning parameter correction in pedestrian navigation system. IEEE Transactions on Instrumentation and Measurement, 3(52): 1554-1558, February 2001. ISSN 0018-9456.

[44] T. Kailath, A. H. Sayed, and B. Hassibi. Linear Estimation. Prentice Hall, 2000. ISBN 978-0-13-022464-4.

[45] R. E. Kalman. New approach to linear filtering and prediction problems. Transactions of the ASME-Journal of Basic Engineering, 82(59):3545, February 1960.

[46] W. Kang and Y. Han. SmartPDR: Smartphone-based pedestrian dead reckoning for indoor localization. IEEE Sensors Journal, 15(6):2906-2916, May 2015.

[47] P. Kasebzadeh. Parameter estimation for mobile positioning applications. Licentiate thesis, Department of Electrical Engineering, Linköping University, 2017.

[48] P. Kasebzadeh, C. Fritsche, G. Hendeby, F. Gunnarsson, and F. Gustafsson. Improved pedestrian dead reckoning positioning with gait parameter learning. In International Conference on Information Fusion, Heidelberg, Germany, July 2016. 
[49] P. Kasebzadeh, G. Hendeby, C. Fritsche, F. Gunnarsson, and F. Gustafsson. IMU dataset for motion and device mode classification. In 8th Internation Conference on Indoor Positioning and Indoor Navigation (IPIN2017), Sapporo, Japan, September 2017.

[50] P Kasebzadeh, G Hendeby, and F. Gustafsson. Asynchronous averaging of gait cycles for classification of gait and device modes. In eprint arXiv: arXiv:1907.02329v2, 2019.

[51] P. Kasebzadeh, K. Radnosrati, G. Hendeby, and F. Gustafsson. Joint pedestrian motion state and device pose classification. 2019.

[52] M. Khedr and N. El-Sheimy. A smartphone step counter using IMU and magnetometer for navigation and health monitoring applications. Sensors, 17(11), November 2017.

[53] D. P. Kingma and J. L. Ba. Adam: A method for stochastic optimization. In International Conference on Learning Representations, volume abs/1412.6980, August 2014.

[54] G. Kitagawa. Non-Gaussian state-space modeling of nonstationary time series. Journal of the American Statistical Association, 82(400):1032-1041, December 1987.

[55] I. Klein, Y. Solaz, and G. Ohayon. Pedestrian dead reckoning with smartphone mode recognition. IEEE Sensors Journal, 18(18):7577-7584, September 2018 .

[56] M. Kok. Probabilistic modeling for sensor fusion with inertial measurements. PhD thesis, Linköping studies in science and technology, 2017.

[57] R. W. Levi and T. Judd. Dead reckoning navigational system using accelerometer to measure foot impacts, 1996.

[58] J. F. Li, Q. H. Wang, X. M. Liu, and M. Y. Zhang. An autonomous waistmounted pedestrian dead reckoning system by coupling low-cost MEMS inertial sensors and GPS receiver for 3D urban navigation. Journal of Engineering Science and Technology, 7(9):9-14, March 2014.

[59] Y. Li, Y. Zhuang, P. Lan, Hand Zhang, X Niu, and N. El-Sheimy. Selfcontained indoor pedestrian navigation using smartphone sensors and magnetic features. IEEE Sensors Journal, 16(19):7173 - 7182, October 2016.

[60] Linköping University, Sweden. Sensor fusion app, December 2014. URL https://goo.gl/0qNyU.

[61] Y. Liu, Y. Chen, L. Shi, Z. Tian, M. Zhou, and L. Li. Accelerometer based joint step detection and adaptive step length estimation algorithm using handheld devices. Journal of Communications, 10(7):520-525, July 2015. 
[62] L. Ljung. System Identification: Theory for the User. Prentice Hall, Upper Saddle River, NJ, USA, 1999. ISBN 978-0-13-656695-3.

[63] H. J. Luinge and P. H. Veltink. Inclination measurement of human movement using a 3-D accelerometer with autocalibration. IEEE Transactions Neural System Rehabil. Eng., 12(1):112-121, March 2004.

[64] MapToaster. How GPS works, 2017. URL https : //goo.gl/khHmxw.

[65] A. Martinelli, H. Gao, P. D. Groves, and S. Morosi. Probabilistic contextaware step length estimation for pedestrian dead reckoning. IEEE Sensors Journal, 18(4):1600-1611, February 2018.

[66] J. Nocedal and S. J. Wright. Nomerical Optimization. Springer, 2006.

[67] A. Norrdine, Z. Kasmi, and J. Blankenbach. Step detection for ZUPT-aided inertial pedestrian navigation system using foot-mounted permanent magnet. IEEE Sensors Journal, 16(17):6766-6773, September 2016.

[68] G. Panahandeh, N. Mohammadiha, A. Leijon, and P. Händel. Continuous hidden Markov model for pedestrian activity classification and gait analysis. IEEE Transactions on Instrumentation and Measurement, 62(5):10731083, May 2013.

[69] I. P. I. Pappas, M. R. Popovic, T. Keller, V. Dietz, and M. Morari. A reliable gait phase detection system. IEEE Transactions on Neural Systems and Rehabilitation, 9(2):113-125, June 2001.

[70] L. Pei, J. Liu, R. Guinness, Y. Chen, H. Kuusniemi, and R. Chen. Using LSSVM based motion recognition for smartphone indoor wireless positioning. Sensors, 12(5):6155-6175, May 2012.

[71] N. Qian. On the momentum term in gradient descent learning algorithms. Neural Networks, 2(1):145-151, January 1999.

[72] L. R. Rabiner. A tutorial on hidden Markov models and selected applications in speech recognition. Proceedings of the IEEE, 77(2):257-286, February 1989.

[73] L. R. Rabiner. First-hand: The hidden Markov model. IEEE Global History Network, October 2013.

[74] C. E. Rasmussen and C. K. I. Williams. Gaussian Processes for Machine Learning. MIT Press, 2006.

[75] H. E. Rauch, F. Tung, and C. T. Striebel. Maximum likelihood estimates of linear dynamic system. AIAA Journals, 3(8):1445-1450, August 1965.

[76] A. Reiss, G. Hendeby, and D. Stricker. A competitive approach for human activity recognition on smartphones. In European Symposium on Artificial Neural Networks, Computational Intelligence and Machine Learning (ESANN), Bruges, Belgium, April 2013. 
[77] C. Ren, T. Fu, M. Zhou, and X. Hu. Low-cost 3D positioning system based on SEMG and MIMU. IEEE Transactions on Instrumentation and Measurement, 67(4):876-884, April 2018.

[78] V. Renaudin, M. Susi, and G. Lachapelle. Step length estimation using handheld inertial sensors. Sensors, 12(7):8507-8525, June 2012.

[79] V. Renaudin, V. Demeule, and M. Ortiz. Adaptative pedestrian displacement estimation with a smartphone. In International Conference on Indoor Positioning and Indoor Navigation (IPIN2013), Calgary, Canada, October 2013.

[80] A. R. J. Ruiz, F. S. Granja, J. C. P. Honorato, and J. I. G. Rosas. Accurate pedestrian indoor navigation by tightly coupling foot-mounted IMU and RFID measurements. IEEE Transactions on Instrumentation and Measurement, 61(1):178-189, January 2012.

[81] S. Särkkä. Bayesian Filtering and Smoothing. Cambridge University Press, 2013.

[82] S. F. Schmidt. Applications of state space methods to navigation problems. Advanced Control Systems, 3:293-340, June 1966.

[83] B. Shin, S. Lee, C. Kim, J. Kim, T. Lee, C. Kee, S. Heo, and H. Rhee. Implementation and performance analysis of smartphone-based 3D PDR system with hybrid motion and heading classifier. In Position, Location and Navigation Symposium (PLANS), July 2014.

[84] B. Shin, C. C. Kim, J. Kim, S. Lee, C. Kee, H. Kim, and T. Lee. Motion recognition-based 3D pedestrian navigation system using smartphone. IEEE Sensors Journal, 16(18):6977-6989, September 2016.

[85] S. H. Shin, C. G. Park, J. W. Kim, H. S. Hong, and J. M. Lee. Adaptive step length estimation algorithm using low-cost MEMS inertial sensors. In Sensors Applications Symposium (SAS '07), San Diego, California, USA, February 2007.

[86] I. Skog, P. Handel, J.O. Nilsson, and J. Rantakokko. Zero-velocity detection-an algorithm evaluation. IEEE Transactions on Biomedical Engineering, 57(11):2657-2666, November 2010.

[87] G. L. Smith, S. F. Schmidt, and L. A. McGee. Application of statistical filter theory to the optimal estimation of position and velocity on board a circumlunar vehicle. Technical report, NASA TR R-135, National Aeronatics and Space Administration, 1962.

[88] N. Srivastava, G. Hinton, A. Krizhevsky, I. Sutskever, and R. Salakhutdinov. Dropout: A simple way to prevent neural networks from overfitting. Journal of Machine Learning Research, 15(2014):1929-1958, June 2014. 
[89] U. Steinhoff and B. Schiele. Dead reckoning from the pocket - an experimental study. In IEEE International Conference on Pervasive Computing and Communications (PerCom), Mannheim, Germany, March 2010.

[90] V. Susi, M. Renaudin and G. Lachapelle. Motion mode recognition and step detection algorithms for mobile phone users. Sensors, 13(2):1539-1562, January 2013.

[91] Q. Tian, K.I-K. Salcic, Z. Wang, and Y. Pan. A multi-mode dead reckoning system for pedestrian tracking using smartphones. IEEE Sensors Journal, 16(7):2079 - 2093, December 2016.

[92] Z. Tian, Y. Zhang, M. Zhou, and Y. Liu. Pedestrian dead reckoning for MARG navigation using a smartphone. EURASIP Journal on Advances in Signal Processing, 16(1):2-11, May 2014.

[93] D. H. Titterton and J. L. Weston. Strapdown inertial navigation technology. Peter Peregrinis Ltd. on behalf of the Institution of Electrical Engineers, 1997.

[94] S. Tomažič and I. Škrjanc. Fusion of visual odometry and inertial navigation system on a smartphone. Elsevier on Computers in Industry, 74: 119-134, December 2015.

[95] Target Tracking. Derivation of the IMM filter, 2014. URL https://goo. gl/MHyNUG.

[96] B. Wang, X. Liu, B. Yu, R. Jia, and X. Gan. Pedestrian dead reckoning based on motion mode recognition using a smartphone. Sensors Journal, 18(6), June 2018.

[97] C. K. I. Williams and D. Barber. Bayesian classification with Gaussian processes. IEEE Transactions on Pattern Analysis and Machine Intelligence, 20(12):1342 - 1351, December 1998.

[98] O. J. Woodman. An introduction to inertial navigation. Technical Report 696, University Of Cambridge, 2007.

[99] MVN Users Manual. User Guide MVN, MVN BIOMECH, MVN Link, MVN Awinda. Xsens, Nov. 2016. URL https://issuu. com/xsensmvn/docs/mvn_user_manual_71c37181653db5?e= $14522406 / 12478179$.

[100] C. C. Yang and Y. L. Hsu. A review of accelerometry-based wearable motion detectors for physical activity monitoring. Sensors, 10(8):7772-7788, August 2010.

[101] Q. Zeng, S. Zeng, J. Liu, Q. Meng, R. Chen, and H. Huang. Smartphone heading correction based on gravity assisted and middle time simulatedzero velocity update method. Sensors, 18(10), October 2018. 
[102] H. Zhang, W. Yuan, Q. Shen, T. Li, and H. Chang. A handheld inertial pedestrian navigation system with accurate step modes and device poses recognition. IEEE Sensors Journal, 15(3):1421-1429, January 2015.

[103] Z. Zhang, Y. Li, C. Peng, D. Mou, M. Li, and W. Wang. The height-adaptive parameterized step length measurement method and experiment based on motion parameters. Journal Sensors, 18(4):1039-1050, March 2018.

[104] H. Zhao, L. Zhang, S. Qiu, Z. Wang, N. Yang, and J. Xu. Pedestrian dead reckoning using pocket-worn smartphone. IEEE Access, 7:91063 - 91073, July 2019.

[105] S. Zihajehzadeh, T. J. Lee, J. K. Lee, R. Hoskinson, and E. J. Park. Integration of mems inertial and pressure sensors for vertical trajectory determination. IEEE Transactions on Instrumentation and Measurement, 64(3):804-814, March 2015. 



\section{PhD Dissertations \\ Division of Automatic Control \\ Linköping University}

M. Millnert: Identification and control of systems subject to abrupt changes. Thesis No. 82, 1982. ISBN 91-7372-542-0.

A. J. M. van Overbeek: On-line structure selection for the identification of multivariable systems. Thesis No. 86, 1982. ISBN 91-7372-586-2.

B. Bengtsson: On some control problems for queues. Thesis No. 87, 1982. ISBN 91-7372593-5.

S. Ljung: Fast algorithms for integral equations and least squares identification problems. Thesis No. 93, 1983. ISBN 91-7372-641-9.

H. Jonson: A Newton method for solving non-linear optimal control problems with general constraints. Thesis No. 104, 1983. ISBN 91-7372-718-0.

E. Trulsson: Adaptive control based on explicit criterion minimization. Thesis No. 106, 1983. ISBN 91-7372-728-8.

K. Nordström: Uncertainty, robustness and sensitivity reduction in the design of single input control systems. Thesis No. 162, 1987. ISBN 91-7870-170-8.

B. Wahlberg: On the identification and approximation of linear systems. Thesis No. 163, 1987. ISBN 91-7870-175-9.

S. Gunnarsson: Frequency domain aspects of modeling and control in adaptive systems. Thesis No. 194, 1988. ISBN 91-7870-380-8.

A. Isaksson: On system identification in one and two dimensions with signal processing applications. Thesis No. 196, 1988. ISBN 91-7870-383-2.

M. Viberg: Subspace fitting concepts in sensor array processing. Thesis No. 217, 1989. ISBN 91-7870-529-0.

K. Forsman: Constructive commutative algebra in nonlinear control theory. Thesis No. 261, 1991. ISBN 91-7870-827-3.

F. Gustafsson: Estimation of discrete parameters in linear systems. Thesis No. 271, 1992. ISBN 91-7870-876-1.

P. Nagy: Tools for knowledge-based signal processing with applications to system identification. Thesis No. 280, 1992. ISBN 91-7870-962-8.

T. Svensson: Mathematical tools and software for analysis and design of nonlinear control systems. Thesis No. 285, 1992. ISBN 91-7870-989-X.

S. Andersson: On dimension reduction in sensor array signal processing. Thesis No. 290, 1992. ISBN 91-7871-015-4.

H. Hjalmarsson: Aspects on incomplete modeling in system identification. Thesis No. 298, 1993. ISBN 91-7871-070-7.

I. Klein: Automatic synthesis of sequential control schemes. Thesis No. 305, 1993. ISBN 91-7871-090-1.

J.-E. Strömberg: A mode switching modelling philosophy. Thesis No. 353, 1994. ISBN 91 7871-430-3.

K. Wang Chen: Transformation and symbolic calculations in filtering and control. Thesis No. 361, 1994. ISBN 91-7871-467-2.

T. McKelvey: Identification of state-space models from time and frequency data. Thesis No. 380, 1995. ISBN 91-7871-531-8.

J. Sjöberg: Non-linear system identification with neural networks. Thesis No. 381, 1995. ISBN 91-7871-534-2.

R. Germundsson: Symbolic systems - theory, computation and applications. Thesis No. 389, 1995. ISBN 91-7871-578-4. 
P. Pucar: Modeling and segmentation using multiple models. Thesis No. 405, 1995. ISBN 91-7871-627-6.

H. Fortell: Algebraic approaches to normal forms and zero dynamics. Thesis No. 407, 1995. ISBN 91-7871-629-2.

A. Helmersson: Methods for robust gain scheduling. Thesis No. 406, 1995. ISBN 91-7871628-4.

P. Lindskog: Methods, algorithms and tools for system identification based on prior knowledge. Thesis No. 436, 1996. ISBN 91-7871-424-8.

J. Gunnarsson: Symbolic methods and tools for discrete event dynamic systems. Thesis No. 477, 1997. ISBN 91-7871-917-8.

M. Jirstrand: Constructive methods for inequality constraints in control. Thesis No. 527, 1998. ISBN 91-7219-187-2.

U. Forssell: Closed-loop identification: Methods, theory, and applications. Thesis No. 566, 1999. ISBN 91-7219-432-4.

A. Stenman: Model on demand: Algorithms, analysis and applications. Thesis No. 571, 1999. ISBN 91-7219-450-2.

N. Bergman: Recursive Bayesian estimation: Navigation and tracking applications. Thesis No. 579, 1999. ISBN 91-7219-473-1.

K. Edström: Switched bond graphs: Simulation and analysis. Thesis No. 586, 1999. ISBN 91-7219-493-6.

M. Larsson: Behavioral and structural model based approaches to discrete diagnosis. Thesis No. 608, 1999. ISBN 91-7219-615-5.

F. Gunnarsson: Power control in cellular radio systems: Analysis, design and estimation. Thesis No. 623, 2000. ISBN 91-7219-689-0.

V. Einarsson: Model checking methods for mode switching systems. Thesis No. 652, 2000. ISBN 91-7219-836-2.

M. Norrlöf: Iterative learning control: Analysis, design, and experiments. Thesis No. 653, 2000. ISBN 91-7219-837-0.

F. Tjärnström: Variance expressions and model reduction in system identification. Thesis No. 730, 2002. ISBN 91-7373-253-2.

J. Löfberg: Minimax approaches to robust model predictive control. Thesis No. 812, 2003. ISBN 91-7373-622-8.

J. Roll: Local and piecewise affine approaches to system identification. Thesis No. 802, 2003. ISBN 91-7373-608-2.

J. Elbornsson: Analysis, estimation and compensation of mismatch effects in A/D converters. Thesis No. 811, 2003. ISBN 91-7373-621-X.

O. Härkegård: Backstepping and control allocation with applications to flight control. Thesis No. 820, 2003. ISBN 91-7373-647-3.

R. Wallin: Optimization algorithms for system analysis and identification. Thesis No. 919, 2004. ISBN 91-85297-19-4.

D. Lindgren: Projection methods for classification and identification. Thesis No. 915, 2005. ISBN 91-85297-06-2.

R. Karlsson: Particle Filtering for Positioning and Tracking Applications. Thesis No. 924, 2005. ISBN 91-85297-34-8.

J. Jansson: Collision Avoidance Theory with Applications to Automotive Collision Mitigation. Thesis No. 950, 2005. ISBN 91-85299-45-6.

E. Geijer Lundin: Uplink Load in CDMA Cellular Radio Systems. Thesis No. 977, 2005. ISBN 91-85457-49-3.

M. Enqvist: Linear Models of Nonlinear Systems. Thesis No. 985, 2005. ISBN 91-8545764-7.

T. B. Schön: Estimation of Nonlinear Dynamic Systems - Theory and Applications. Thesis No. 998, 2006. ISBN 91-85497-03-7. 
I. Lind: Regressor and Structure Selection - Uses of ANOVA in System Identification. Thesis No. 1012, 2006. ISBN 91-85523-98-4.

J. Gillberg: Frequency Domain Identification of Continuous-Time Systems Reconstruction and Robustness. Thesis No. 1031, 2006. ISBN 91-85523-34-8.

M. Gerdin: Identification and Estimation for Models Described by Differential-Algebraic Equations. Thesis No. 1046, 2006. ISBN 91-85643-87-4.

C. Grönwall: Ground Object Recognition using Laser Radar Data - Geometric Fitting, Performance Analysis, and Applications. Thesis No. 1055, 2006. ISBN 91-85643-53-X.

A. Eidehall: Tracking and threat assessment for automotive collision avoidance. Thesis No. 1066, 2007. ISBN 91-85643-10-6.

F. Eng: Non-Uniform Sampling in Statistical Signal Processing. Thesis No. 1082, 2007. ISBN 978-91-85715-49-7.

E. Wernholt: Multivariable Frequency-Domain Identification of Industrial Robots. Thesis No. 1138, 2007. ISBN 978-91-85895-72-4.

D. Axehill: Integer Quadratic Programming for Control and Communication. Thesis No. 1158, 2008. ISBN 978-91-85523-03-0.

G. Hendeby: Performance and Implementation Aspects of Nonlinear Filtering. Thesis No. 1161, 2008. ISBN 978-91-7393-979-9.

J. Sjöberg: Optimal Control and Model Reduction of Nonlinear DAE Models. Thesis No. 1166, 2008. ISBN 978-91-7393-964-5.

D. Törnqvist: Estimation and Detection with Applications to Navigation. Thesis No. 1216, 2008. ISBN 978-91-7393-785-6.

P-J. Nordlund: Efficient Estimation and Detection Methods for Airborne Applications. Thesis No. 1231, 2008. ISBN 978-91-7393-720-7.

H. Tidefelt: Differential-algebraic equations and matrix-valued singular perturbation. Thesis No. 1292, 2009. ISBN 978-91-7393-479-4.

H. Ohlsson: Regularization for Sparseness and Smoothness - Applications in System Identification and Signal Processing. Thesis No. 1351, 2010. ISBN 978-91-7393-287-5.

S. Moberg: Modeling and Control of Flexible Manipulators. Thesis No. 1349, 2010. ISBN 978-91-7393-289-9.

J. Wallén: Estimation-based iterative learning control. Thesis No. 1358, 2011. ISBN 978 91-7393-255-4.

J. D. Hol: Sensor Fusion and Calibration of Inertial Sensors, Vision, Ultra-Wideband and GPS. Thesis No. 1368, 2011. ISBN 978-91-7393-197-7.

D. Ankelhed: On the Design of Low Order H-infinity Controllers. Thesis No. 1371, 2011. ISBN 978-91-7393-157-1.

C. Lundquist: Sensor Fusion for Automotive Applications. Thesis No. 1409, 2011. ISBN 978-91-7393-023-9.

P. Skoglar: Tracking and Planning for Surveillance Applications. Thesis No. 1432, 2012. ISBN 978-91-7519-941-2.

K. Granström: Extended target tracking using PHD filters. Thesis No. 1476, 2012. ISBN 978-91-7519-796-8.

C. Lyzell: Structural Reformulations in System Identification. Thesis No. 1475, 2012. ISBN 978-91-7519-800-2.

J. Callmer: Autonomous Localization in Unknown Environments. Thesis No. 1520, 2013. ISBN 978-91-7519-620-6.

D. Petersson: A Nonlinear Optimization Approach to H2-Optimal Modeling and Control. Thesis No. 1528, 2013. ISBN 978-91-7519-567-4.

Z. Sjanic: Navigation and Mapping for Aerial Vehicles Based on Inertial and Imaging Sensors. Thesis No. 1533, 2013. ISBN 978-91-7519-553-7. 
F. Lindsten: Particle Filters and Markov Chains for Learning of Dynamical Systems. Thesis No. 1530, 2013. ISBN 978-91-7519-559-9.

P. Axelsson: Sensor Fusion and Control Applied to Industrial Manipulators. Thesis No. 1585, 2014. ISBN 978-91-7519-368-7.

A. Carvalho Bittencourt: Modeling and Diagnosis of Friction and Wear in Industrial Robots. Thesis No. 1617, 2014. ISBN 978-91-7519-251-2.

M. Skoglund: Inertial Navigation and Mapping for Autonomous Vehicles. Thesis No. 1623, 2014. ISBN 978-91-7519-233-8.

S. Khoshfetrat Pakazad: Divide and Conquer: Distributed Optimization and Robustness Analysis. Thesis No. 1676, 2015. ISBN 978-91-7519-050-1.

T. Ardeshiri: Analytical Approximations for Bayesian Inference. Thesis No. 1710, 2015. ISBN 978-91-7685-930-8.

N. Wahlström: Modeling of Magnetic Fields and Extended Objects for Localization Applications. Thesis No. 1723, 2015. ISBN 978-91-7685-903-2.

J. Dahlin: Accelerating Monte Carlo methods for Bayesian inference in dynamical models. Thesis No. 1754, 2016. ISBN 978-91-7685-797-7.

M. Kok: Probabilistic modeling for sensor fusion with inertial measurements. Thesis No. 1814, 2016. ISBN 978-91-7685-621-5.

J. Linder: Indirect System Identification for Unknown Input Problems: With Applications to Ships. Thesis No. 1829, 2017. ISBN 978-91-7685-588-1.

M. Roth: Advanced Kalman Filtering Approaches to Bayesian State Estimation. Thesis No. 1832, 2017. ISBN 978-91-7685-578-2.

I. Nielsen: Structure-Exploiting Numerical Algorithms for Optimal Control. Thesis No. 1848, 2017. ISBN 978-91-7685-528-7.

D. Simon: Fighter Aircraft Maneuver Limiting Using MPC: Theory and Application. Thesis No. 1881, 2017. ISBN 978-91-7685-450-1.

C. Veibäck: Tracking the Wanders of Nature. Thesis No. 1958, 2018. ISBN 978-91-7685200-2.

C. Andersson Naesseth: Machine learning using approximate inference: Variational and sequential Monte Carlo methods. Thesis No. 1969, 2018. ISBN 978-91-7685-161-6.

Y. Jung: Inverse system identification with applications in predistortion. Thesis No. 1966, 2018. ISBN 978-91-7685-171-5.

Y. Zhao: Gaussian Processes for Positioning Using Radio Signal Strength Measurements. Thesis No. 1968, 2019. ISBN 978-91-7685-162-3.

R. Larsson: Flight Test System Identification. Thesis No. 1990, 2019. ISBN 978-91-7685070-1. 


\section{FACULTY OF SCIENCE AND ENGINEERING}

Linköping Studies in Science and Technology, Dissertation No. 2012, 2019 Department of Electrical Engineering

Linköping University

SE-58183 Linköping, Sweden

www.liu.se

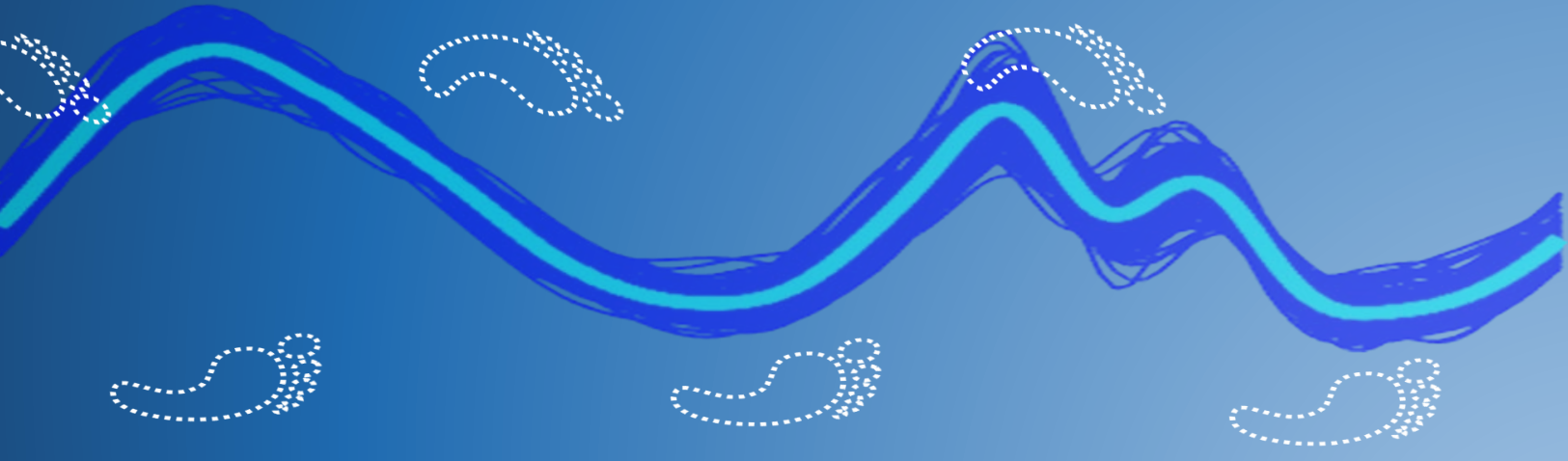

